\title{
Mathematical aspects of catalyst positioning in Lithium/air batteries
}

\author{
M.Sc. Thuong Huyen Nguyen
}

\section{Dissertation}

zur Erlangung des Grades eines Doktors

der Naturwissenschaften

- Dr. rer. nat. -

Vorgelegt im Fachbereich 3

Universität Bremen 
Datum des Promotionskolloquiums: 12th December 2018

Gutachter: Prof. Dr. Peter Maass, Universität Bremen, Germany

Prof. Dr. Dinh Nho Hào, Institute of Mathematics, Vietnam 


\section{Acknowledgements}

Fist of all, I would like to express my deep gratitude to my supervisors Prof. Dr. Peter Maass and Prof. Dr. Dinh Nho Hào for their patience, support and valuable advice during my $\mathrm{PhD}$ period. Their guidance helped me in all the time of doing research and writing this thesis. I would not overcome the dificulties without their help and encouragement.

I would like to express my sincere appreciation to Prof. Dr. Michael Boehm for his precious suggestions on the fixed-point method at the first step of my thesis. I am very grateful to Dr. Tatjiana Dabrowski for her kind help on $\mathrm{C}++$ programming and disscusion about the greedy algorithm. Based mainly on her code, I developed the programs in this thesis.

I would like to thank „Project 911“, Department of Education and Training, Vietnam for supporting the $\mathrm{PhD}$ scholarship during my study in Germany. Besides, Prof. Dr. Peter Maass and Center of Industrial Mathematics, University of Bremen also fund me for conducting my research, I would like to deeply thank them.

I sincerely thank all members of Center for Industrial Mathematics ZeTeM, University of Bremen for providing a nice environment for researching and living. I gratefully thank Simon, Delf, Christine, Yovany, Phil, Patrick, Tobias for their sincere help and interesting talk not only about work but also about personal life. I am grateful to Victor for his precious IT help. I also would like to thank Judith and Doerte for their great help during my time in Bremen.

Last but not least, I would like to express my sincere gratitude to my family, my fiance, my best friends for their unconditional love and support during my tough time in my $\mathrm{PhD}$ pursuit.

Bremen, 18th of November, 2018

Nguyen Thuong Huyen 



\begin{abstract}
Lithium-Luft-Akkumulatoren haben in den letzten Jahren das Interesse vieler Wissenschaftler auf sich gezogen. Das Problem der Katalysatorpositionierung, welches die poröse Kathode während des Entladungsprozesses beschreibt, ist Teil der Forschung zur Optimierung der Kapazität von Lithium-Luft-Akkumulatoren. Während des Entladungsprozesses kommt es zu einem schwerwiegenden Problem: Die Sauerstoffreduktionsprodukte der Entladung sind in den organischen Elektrolyten unlöslich. Dadurch wird der Zufluss von Sauerstoff für die Reaktion mit Lithiumionen in die Pore verstopft und die Kapazität der Batterien begrenzt, weil die aktive Oberfläche innerhalb der Pore verengt wird. Die Dynamik des Entladungsprozesses wird durch ein Anfangs- und inhomogenes Randwertproblem für zwei eindimensionale partielle Differenzialgleichungen beschrieben. Die zwei Variablen des Systems sind der Porenradius innerhalb der Kathode und die Sauerstoffkonzentration zu bestimmten Koordinaten und Zeitpunkten.

Gegenstand der Arbeit ist es, einige Katalysatorpositionierungsmodelle zu untersuchen und das freie Volumen der Pore nach dem Verstopfen der Poren durch die abgelagerten Entladungsprodukte zu maximieren. Die Arbeit konzentriert sich auf die folgenden Bereiche:

Erstens untersuchen wir analytisch das Vorwärtsmodell, das die stetige Positionierung der Katalysatoren von Lithium-Luft-Akkumulatoren beschreibt. die korrekte Problemstellung, die Fréchet-Differenzierbarkeit des Porenradius und der Sauerstoffkonzentration in Abhängigkeit zur Katalisatorfunktion in einigen Räumen.

Zweitens stellen wir Optimierungsprobleme vor und analysieren die Sensitivitäts- und Adjoint-Methode, um sie zu lösen.

Schließlich werden einige numerische Methoden zur Lösung der Vorwärtsprobleme durchgeführt, und einige numerische Ansätze zur Lösung des Optimierungsproblems werden ebenfalls untersucht, um die theoretischen Ergebnisse zu veranschaulichen.
\end{abstract}





\begin{abstract}
Lithium/air batteries has been taken interest by many scientists over the last years. The catalyst positioning problem describing the porous cathode during the discharge process is concerned in the research of optimizing the capacity of Lithium/air batteries. During discharge process, there is a critical issue: the discharge oxygen reduction products is insoluble in the organic electrolytes. This clogs the oxygen entrance to the pore to be reacted with Lithium ions and limits the capacity of the batteries by narrowing the active surface inside the pore. The dynamics of the discharge process is described by the initial mixed boundary value problem for two one-dimensional partial differentiable equations. The two variables of the system are the pore radius inside the cathode and the oxygen concentration at certain coordinate and time.

The subject of the thesis is to investigate some catalyst positioning models and to maximize the free volume of the pore after pore clogging by the deposited discharged products. We aim at the following fields:

First, we research analytically the forward model describing continuous catalyst positioning in Li/air batteries: well-posedness of the problems, the Fréchet differentiability of the pore radius and the oxygen concentration with respect to catalytic function in some spaces.

Second, we present optimization problems and analyze the sensitivity and adjoint method to solve them.

Finally, some numerical methods are carried out to solve the forward problems and some numerical approach for solving optimization problem are also examined to illustrate the theoretical results.
\end{abstract}





\section{Contents}

Contents vii

List of Figures $\quad$ xi

1 Introduction 1

$\begin{array}{ll}\text { Introduction } & 1\end{array}$

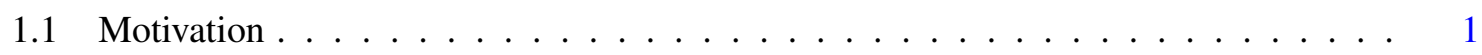

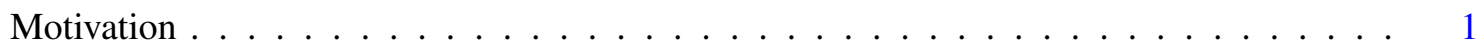

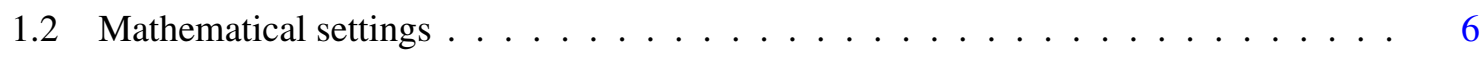

1.2.1 Mathematical setting of the diffusion-limited model for Li/air batteries . . . . 7

1.2.2 Optimization Problems on catalyst positioning models . . . . . . . . . . 14

1.2.2.1 The first optimization problem . . . . . . . . . . . . 14

1.2.2.2 The second optimization problems . . . . . . . . . . 15

1.3 The main results of the Thesis $\ldots \ldots \ldots \ldots \ldots$

2 Mathematical preliminaries $\quad 19$

2.1 Elementary concepts for PDEs . . . . . . . . . . . . . . . . . . . . . . . . . . 19

$2.1 .1 \quad$ Sobolev spaces . . . . . . . . . . . . . . . . . . . . . 19

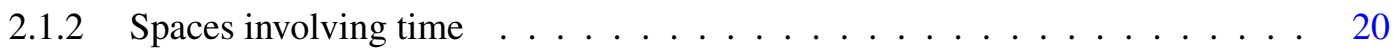

2.2 Mixed boundary value problem for one-dimensional linear parabolic equations _ . . 23

2.3 Convex analysis . . . . . . . . . . . . . . . . . . . . . . . 29

3 Mathematical models for continuous catalyst positioning in Li/air batteries 31 
3.1 Formulation and weak solution of Problem $I_{a} \ldots \ldots \ldots \ldots \ldots$

3.2 The local existence and uniqueness of the weak solution of problem $I_{a} \ldots \ldots \ldots$

3.2.1 $\mathcal{T}$ is well defined in $\boldsymbol{M}_{T}$ and maps $\boldsymbol{M}_{T}$ into $\mathbf{X}_{T} \times \mathbf{Y}_{T}$ for $\mathrm{T}$ small enough . . 35

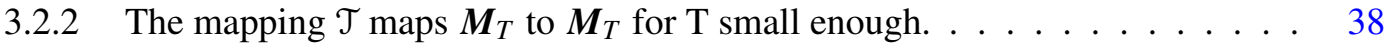

3.2.3 $\mathcal{T}$ is contractive in the $\mathbf{X}_{T} \times \mathbf{Y}_{T}$ norm with $\mathrm{T}$ small enough. $\ldots \ldots \ldots . .40$

3.2.4 The continuous dependency on the data of the weak solution in $\boldsymbol{M}_{T} \ldots \ldots \quad 45$

3.3 The continuity of $(r, c)$ in $W^{1, \infty}\left(Q_{T}\right) \times \mathbf{Y}_{T}$ with respect to $\operatorname{cat}(x)$ in $W^{1, \infty}(\Omega)$ for problem $I_{a}$ with $T$ small enough. $\ldots \ldots \ldots \ldots \ldots$

3.4 On the Frechet differentiability of $r$ and $c$ with respect to $\operatorname{cat}(x)$ for problem $I_{a} \ldots \ldots$

3.4 .1 The sensitivity system $\ldots \ldots \ldots \ldots \ldots$

3.5 Formulation and weak solution of problem $I_{b} \ldots \ldots \ldots \ldots \ldots \ldots \ldots \ldots$

3.6 Existence and uniqueness of the weak solution of problem $I_{b}$ with cat $(\mathrm{t}, \mathrm{x})$ model $\ldots 67$

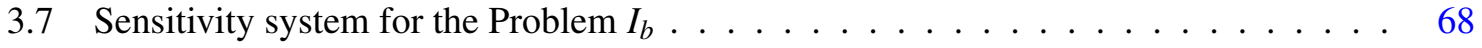

3.8 Formulation and weak solution of the problem $I I_{a} \ldots \ldots \ldots \ldots \ldots$

4 Optimization Problems $\quad 75$

4.1 The first optimization problem . . . . . . . . . . . . . . . . . . 75

4.1.1 Frechet differentiability of the objective functional with respect to catalytic

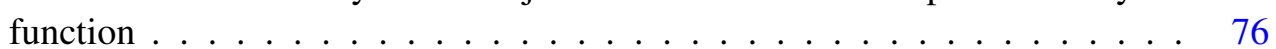

4.1.2 The existence of the solution of the first optimization problem . . . . . . . 77

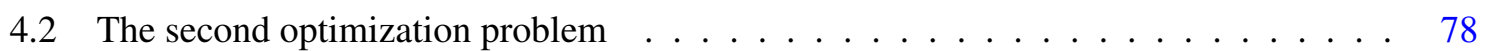

4.2.1 Calculation of the adjoint operator $r_{c a t}^{*}$ for Problem $I_{a} \ldots \ldots \ldots$

4.2.2 Calculation of the adjoint operator $r_{c a t}^{*}$ for Problem $I_{b} \ldots \ldots \ldots$

5 Numerical Implementation $\quad 91$

$5.1 \quad$ Forward Problems . . . . . . . . . . . . . . . . . . . . . . . . . 91

5.1.1 Piecewise basic functions of the spaces $V_{h} \ldots \ldots \ldots \ldots$

5.1.2 The $\theta$ scheme for numerical solving the problems $I$ and $I I \ldots \ldots \ldots$

5.1.3 Comparison between the implicit FDM and the FEM. . . . . . . . . . . . . . 99

5.2 Some remarks on the Greedy algorithm in Problem $I_{b}$ and $I_{b}$ using FDM. . . . . . 102

5.3 The continuous positioning . . . . . . . . . . . . . . . . . . . . . 104

5.3.1 Transformation of discrete catalytic functions to smooth catalytic functions . 107 
5.3.2 cat $(\mathrm{x})$ model $\left(\right.$ Problems $I_{a}$ and $\left.I I_{a}\right) \ldots \ldots \ldots \ldots \ldots \ldots \ldots$

5.3.3 cat $(\mathrm{t}, \mathrm{x})$ model (Problems $I_{b}$ and $\left.I_{b}\right) \ldots \ldots \ldots \ldots \ldots \ldots$

5.3.4 Comparing the two catalyst positioning models: $\operatorname{cat}(\mathrm{x})$ and $\operatorname{cat}(\mathrm{t}, \mathrm{x}) \ldots \ldots 111$

5.4 The optimization problems $\ldots \ldots \ldots \ldots \ldots \ldots \ldots \ldots \ldots$

5.4.1 The first optimization problem for Problem $I_{a} \ldots \ldots \ldots \ldots \ldots \ldots$

5.4 .2 The second optimization problem $\ldots \ldots \ldots \ldots \ldots$

$\begin{array}{ll}\text { Conclusions } & 117\end{array}$

A Elementary Theorems, Lemmas and other concepts 119

$\begin{array}{ll}\text { Appendix } & 119\end{array}$

A.1 Elementary Lemmas and Theorems _ . . . . . . . . . . . . . . . . . . 119

A.2 Huber functions . . . . . . . . . . . . . . . . . . . . . . 120

A.2.1 Continuously differentiable Huber $C^{1}$ function $\ldots \ldots$. . . . . . . . . 120

A.2.2 Continuously differentiable $C^{2}$ Huber function . . . . . . . . . . . . . 122

A.3 The Gaussian distribution (Normal distribution) . . . . . . . . . . . . . . . . . . . 123

$\begin{array}{ll}\text { APPENDICES } & 118\end{array}$

$\begin{array}{lr}\text { Bibliography } & 127\end{array}$ 



\section{List of Figures}

1.1 Characteristics of some metal/oxygen battery couples adapted from [32] . . . . . . 1

1.2 Oxygen solubility $C_{O_{2}, 0}$ and diffusivity $D_{O_{2}}$ of the four considered electrolytes. . . 2

1.3 A simple sketch of lithium air batteries adopted from [32] . . . . . . . . . . . . 3

1.4 The radius of pore with different electrolytes before pore clogging (the radius reaches to 0.1$). \mathrm{Li}_{2} \mathrm{O}_{2}$ is deposited in here in the upper side. . . . . . . . . . . 5

1.5 The growth profile of $\mathrm{Li}_{2} \mathrm{O}_{2}$ with centered-catalyst positioning, refered from [8] . . . 5

1.6 (a) Evolution of the minimal free volume after pore clogging as a function of the number of placed catalysts along the pore axis in $D M S \mathrm{O}^{\mathrm{i}^{+}}$for a threshold of $6 \%$. The red line indicates the minimal free volume obtained with the continuous growth model. The numbers of catalysts corresponding to $90 \%, 99 \%, 100 \%$ of the final occupied volume are marked in red. (b) Optimal distributions of catalysts along the pore axis corresponding to the three different cases marked in (a). This Figure is

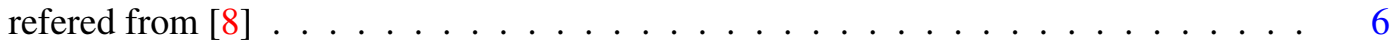

1.7 Schematic representation of the modeled cathode geometry with porosity $\epsilon$ and tortuosity $\tau$ adapted from $[8] \ldots \ldots \ldots \ldots$. . . . . . . . . . . . 8

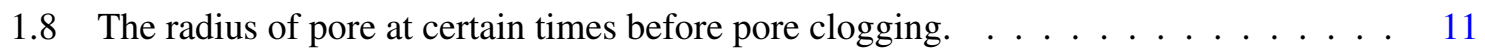

5.1 The subdivision of $\Omega=[0,1], x_{0}=0, x_{J-1}=1 \ldots \ldots \ldots \ldots$

5.2 The piecewise linear finite element basis function $\Phi_{i}(x), i \in[1, J-3] \ldots \ldots$

5.3 The piecewise linear finite element basis function $\Phi_{J-2}(x) \ldots \ldots \ldots$

5.4 The pore radii and the concentration of oxygen inside pore before pore clogging with $J=N=100$ solving with FDM and FEM . . . . . . . . . . . . . . 101

5.5 Free pore volume, relative deviation and runtime for the implicit and explicit FDM . 101

5.6 The pore radii and the concentration of oxygen inside pore before pore clogging with $J=100$ and different $\mathrm{N}$ in Problem I . . . . . . . . . . . . . . . . . 103

5.7 The optimal numbers of catalysts from 1 to 100 by Greedy algorithm in Problem $I I_{b} \quad 104$ 
5.8 Comparison of radii and concentration inside the pore with two optimal numbers of catalyst before pore clogged by Greedy algorithm in Problem $I I_{b} \ldots \ldots \ldots$

5.9 radii and concentration inside the pore with 47 optimal number catalyst by Greedy algorithm in Problem $I_{b} \ldots \ldots \ldots \ldots$

5.10 The distribution of optimal number of catalysts of 47 catalyst positions . . . . . . . . 107

5.11 radii and concentration inside the pore $i$ line plotting with 41 optimal number catalyst by Greedy algorithm in Problem $I_{b} \ldots \ldots \ldots \ldots$

5.12 The distribution of optimal number of catalysts of 41 catalyst positions in Problem $I_{b} \quad 109$

5.13 The transformation from the local optimal discrete catalytic functions by Greedy algorithm in Problem $I I_{b}$ before pore reaches to 0.1 and 0.86 to continuous ones, with $\sigma_{\text {trans }}=0$ in $(5.49) \ldots \ldots \ldots \ldots \ldots \ldots$

5.14 The transformation from local optimal discrete catalytic functions by Greedy algorithm in Problem $I I_{b}$ before pore reaches to 0.1 and 0.86 to continuous ones, with

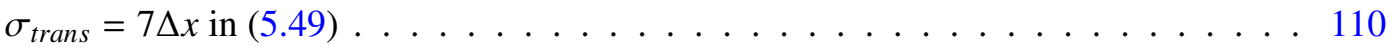

5.15 Comparing the two catalyst positioning models, experimenting with the center-catalyst positioning. The crosses show the catalytic function at the initial time. Blue lines present the growth profiles of discharged product $\mathrm{Li}_{2} \mathrm{O}_{2}$ deposited along the pore surface after pore clogging in Problem $I_{a}$. The red lines are the growth of $\mathrm{Li}_{2} \mathrm{O}_{2}$ according to Problem $I_{b} \ldots \ldots \ldots \ldots \ldots$. . . . . . . . . . . . 111

5.16 Initial $\operatorname{cat}(x)$ and optimal $\operatorname{cat}(x)$ for Problem $I_{a}$ in grid of $J=N=20 \ldots \ldots \ldots$

5.17 Adjoint method and Greedy method with $\mathrm{J}=100, \mathrm{~N}=80$, number of time steps $\mathrm{M}=1000000.115$

5.18 Adjoint method and by Greedy method in Problem $I_{a}$ with $\mathrm{J}=100, \mathrm{~N}=60$, number of time steps $\mathrm{M}=1000000$. The $\mathrm{Li}_{2} \mathrm{O}_{2}$ is deposited in the upper side in this plot . . . . . 116

A.1 The graphic of Huber $C^{1}$ function $h_{1, \gamma}(x)$ with $g=1$ and $\gamma=2 \ldots \ldots \ldots 121$

A.2 The graphic of $C^{1}$ Huber function $H_{1, \gamma, R_{0}}(x)$ with $g=1 / 2, \gamma=50$, and $R_{0}=0.94 \ldots 122$

A.3 Graphic of $C^{2}$ Huber function with $\gamma=2 \ldots \ldots \ldots \ldots \ldots$

A.4 Graphic of $C^{2}$ function $H_{2, \gamma}(x)$ with $\gamma=2, \epsilon=\frac{1}{\gamma}, c_{0}=\frac{1}{\gamma} \ldots \ldots \ldots \ldots \ldots$

A.5 The normal density $f(x) \quad \ldots \ldots \ldots \ldots \ldots \ldots$ 


\section{Chapter 1}

\section{Introduction}

\subsection{Motivation}

Portable electronic devices play important roles in our daily modern life. It is imperative to research the efficient and environmentally friendly way of storing and delivering energy. Lithium/air batteries recently attract great attention of many engineers and scientists because of many advantages. They have higher potential theoretical energy values in comparison with the other metal/air batteries as shown in 1.1. Besides, with a porous structure cathode, they also are the good candidate of the lighter power sourse for aerial vehicales, camping equipments compared with ion-Lithium baterry [32]. There are basically two kinds of Lithium/ air batteries: aqueous and non-aqueous. The nonaqueous differs from the aqueous Lithium/air batteries in that the discharged products are insoluble in the aqueous electrolytes, [1], [29], [30], which leads to the pore's passivation. However, the aqueous Lithium/air batteries require an isolated protection layer between metallic Lithium and the electrolyte, that will significantly limits the $\mathrm{Li}^{+}$- conductivity. For this difficulty, most of literatures concerning on Lithium/air batteries studying on the non-aqueous electrolyte baterries. For a detail research on the capacities of aqueous, non-aqueous Li/air and Li-ion batteries we refer to the article of Christensen et al. [5].

The main reactions in non-aqueous electrolyte $\mathrm{Li} /$ air batteries is given by

$$
2 \mathrm{Li}+\mathrm{O}_{2} \leftrightarrows \mathrm{Li}_{2} \mathrm{O}_{2}
$$

\begin{tabular}{|c|c|c|c|c|}
\hline \multirow[t]{2}{*}{ Metal $/ \mathrm{O}_{2}$ couple } & \multirow[t]{2}{*}{ Ideal cell reaction $^{\mathrm{a}}$} & \multirow{2}{*}{$\begin{array}{l}\text { Calculated open-circuit } \\
\text { voltage at } 25^{\circ} \mathrm{C}(\mathrm{V})\end{array}$} & \multicolumn{2}{|c|}{ Theoretical specific energy ${ }^{\mathrm{b}}\left(\mathrm{Wh} \mathrm{kg}^{-1}\right)$} \\
\hline & & & Including $\mathrm{O}_{2}$ & Excluding $\mathrm{O}_{2}$ \\
\hline $\mathrm{Li} / \mathrm{O}_{2}$ & $4 \mathrm{Li}+\mathrm{O}_{2} \rightleftharpoons 2 \mathrm{Li}_{2} \mathrm{O}$ & 2.91 & 5200 & 11,140 \\
\hline $\mathrm{Al} / \mathrm{O}_{2}$ & $4 \mathrm{Al}+3 \mathrm{O}_{2} \rightleftharpoons 2 \mathrm{Al}_{2} \mathrm{O}_{3}$ & 2.73 & 4300 & 8,130 \\
\hline $\mathrm{Ca} / \mathrm{O}_{2}$ & $2 \mathrm{Ca}+\mathrm{O}_{2} \rightleftharpoons 2 \mathrm{CaO}$ & 3.12 & 2990 & 4,180 \\
\hline $\mathrm{Zn} / \mathrm{O}_{2}$ & $2 \mathrm{Zn}+\mathrm{O}_{2} \rightleftharpoons 2 \mathrm{ZnO}$ & 1.65 & 1090 & 1,350 \\
\hline
\end{tabular}

a The reduction of $\mathrm{O}_{2}$ to $\mathrm{O}^{2-}$ usually occurs only in the presence of a catalyst; often the product is the peroxide, $\mathrm{O}_{2}{ }^{2-}$.

${ }^{b}$ Includes only the active materials. Since $\mathrm{O}_{2}$ does not have to be carried in the battery, values are given for the cases of including and excluding $\mathrm{O}_{2}$. The battery weight will increase once the discharge begins.

Figure 1.1: Characteristics of some metal/oxygen battery couples adapted from [32] 


\begin{tabular}{lrr}
\hline & $c_{\mathrm{O}_{2}, 0}\left[10^{-6} \frac{\mathrm{mol}}{\mathrm{cm}^{3}}\right]$ & $D_{\mathrm{O}_{2}}\left[10^{-6} \frac{\mathrm{cm}^{2}}{\mathrm{~s}}\right]$ \\
\cline { 1 - 3 } $\mathrm{PC}^{\mathrm{Li}^{+}}$ & 3.20 & 2.24 \\
$\mathrm{TEGDME}^{\mathrm{Li}}{ }^{+}$ & 4.43 & 2.17 \\
$\mathrm{DMSO}^{\mathrm{Li}^{+}}$ & 2.10 & 16.70 \\
$\mathrm{DME}^{\mathrm{Li}}$ & 9.56 & 12.20 \\
\hline
\end{tabular}

Figure 1.2: Oxygen solubility $C_{O_{2}, 0}$ and diffusivity $D_{O_{2}}$ of the four considered electrolytes.

Laoire et al. in the articles [23] and [24] assume that the oxygen reduction reactions (ORR) at the cathode surface during the discharge process, oxygen is consumed by its reaction with Lithium ion and generating the insoluble lithium peroxide as

$$
\begin{aligned}
& \mathrm{O}_{2}+e^{-} \longrightarrow \mathrm{O}_{2}^{-} \\
& \mathrm{O}_{2}^{-}+\mathrm{Li}^{+} \longrightarrow \mathrm{LiO}_{2} \\
& 2 \mathrm{LiO}_{2} \longrightarrow \mathrm{Li}_{2} \mathrm{O}_{2}+\mathrm{O}_{2},
\end{aligned}
$$

and the oxygen evolution reaction (OER)has been formulated to be a direct decomposition process of $\mathrm{Li}_{2} \mathrm{O}_{2}$ according to

$$
\mathrm{Li}_{2} \mathrm{O}_{2} \longrightarrow 2 \mathrm{Li}^{+}+2 e^{-}+\mathrm{O}_{2}
$$

However there are a number of fundamental and practical problems requiring careful investigation. In [32] and [8], these are mentioned as: the role of some specific catalysts in promoting the cathode electrode reaction, optimization of electrode porosity, the structure of the porous cathode, the composition of the cathode electrode, and the prevention of water or carbon dioxide from entering the cell when operated in air. According to [1], [29] and [30], a critical difficulty that we have to overcome is that the discharged oxygen reduction products is insoluble in the organic electrolytes. The low oxygen solubility causes the deposition of the discharged products to the oxygen transporting side as written in [32] and [8] via diffusion-limited model and in [30] via steady-state model.

This clogs the oxygen entrance to the pore to be reacted with Lithium ions and limits the capacity of the batteries by narrowing the active surface inside the pore. There are a number of works that attempt to study this limitation of the batteries' capacity like [41], [29], [3], [25]. They have shown that the specific capacity is enhanced when the current density is decreased. However, in order to support the battery performance and optimize the charging time, the current density should be kept high. Overall, we have to focus on investigating the thickness, porosity and the oxygen solubility 


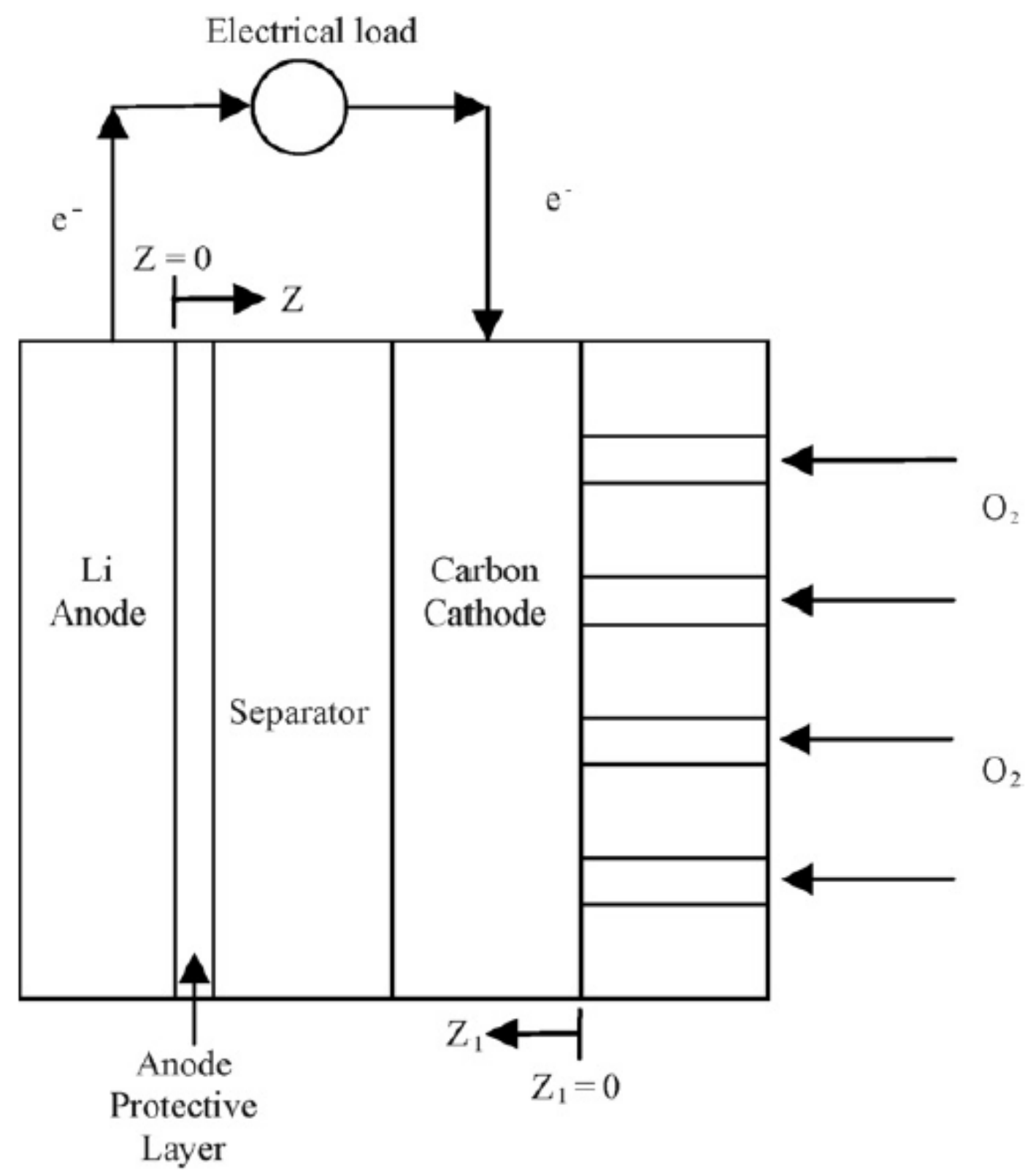

Figure 1.3: A simple sketch of lithium air batteries adopted from [32]. 
and diffusivity in different electrolytes [30] and [36]. The study of [8] aims at optimizing the activity and catalytic distribution at the active surface of the cathode to maximize the free volume of the pore after pore clogging by the deposited discharged products. Besides, the suggested Greedy algorithm of [8] based on a discrete propagation model to express the $\mathrm{Li}_{2} \mathrm{O}_{2}$ growth profile inside the pore is quite advantaged for comparing the effect of the performance among different electrolytes: $P C^{L i^{+}}, T E G D M E^{L i^{+}}, D M E^{L i^{+}}$and $D M S O^{L i^{+}}$. Electrolytes are charaterized by its oxygen solubity $c_{O_{2}, 0}$ and oxygen diffusity $D_{O_{2}}$ of the four considered electrolytes are shown at 1.3. In conclusion, $D M E^{L i^{+}}$and $D M S O^{L i^{+}}$show up the highest specific capacity (actually $D M E^{L i^{+}}$is higher in this term); however $D M S O^{L i^{+}}$,s advantage is the electrochemical stability especially toward the species being produced during the charging process (cf. [9]). For this reason, we choose $D M S O^{L i^{+}}$as a good candidate electrolyte for our numerical experiments and our models for continuous catalytic model.

In this work, we aim at maximizing the capacity of the batteries by optimal catalyst positioning. We propose some reasonable distribution of catalyst along the porous cathode to optimize the utilization pore volume of the cathode of the batteries during oxygen reduction process. Our motivation is taken from the work of Dabrowskji et al [8]. The authors focus on optimizing and positioning of catalytically active sites within the pores to maximize the volume of discharge products before pore clogging. The Figure 1.4 shows the radius of the pore before pore's passivation with different electrolytes. In order to optimize the pore volume utilization, they use a simple propagation scheme to describe the active deposition of the discharge product around its neighborhood. In that work, the authors propose a discrete catalyst model for the discharge process, where the catalytic function taking the value of 1 (switch on) if the radius of the deposited oxygen reduction products of the pore at the neighborhood points reach to a specific threshold, or the value of 0 (switch off) if the radius of discharged product below than that threshold. The difussion-limited mathematical model demonstrating the dynamic of this oxygen reduction progress that they use for the discrete calatyst model is based on the paper from Sandhu et al. [32]. Lately, they optimize the number of the distributed catalyst positions by mean of the greedy method which is used widely in engineering.

In the left graph of 1.5, the authors in [8] simulate the discrete propagation's evolution of the posited amount of $\mathrm{Li}_{2} \mathrm{O}_{2}$ inside the pore with center-catalyst positioning with different thresholds. Those thresholds correspond to different kinetic growth, from the island forming for large threshold (VolmerWeber growth) to a layer-by-layer growth for small thresholds (Frank-van-der-Merwe growth). Later in our time-dependent catalytic model, we also show those relative growth kinetics mechanisms. In the right hand side of Figure 1.5, it is the growth profile time evolution of $\mathrm{Li}_{2} \mathrm{O}_{2}$ with threshold of $4 \%-6 \%$, the one that shows the more natural growth of oxygen reduce reaction product.

Then the maximal utilization of the cathode capacity is carried out using the greedy algorithm, denoting $V_{\text {free }}$ free volume of a pore after clogging and consider it as the objective function. In this algorithm, a catalyst function is supposed to be a "switch on-off" function, that takes the values of either 0 or 1 . This algorithm is implemented iteratively, whose every single step, an optimal catalyst position is sought and will be kept in the next step, with adding a new point with catalyst on. The new optimal catalyst position will be solved out in the next step, and the greedy algorithm will be stopped to be implemented until the next combination of catalyst show a greater the free volume $V_{\text {free }}$ inside the pore. Figure 1.6 shows the optimal number and optimal distribution of catalyst along the pore axis in $D M S O^{L i^{+}}$for a threshold of $6 \%$. 


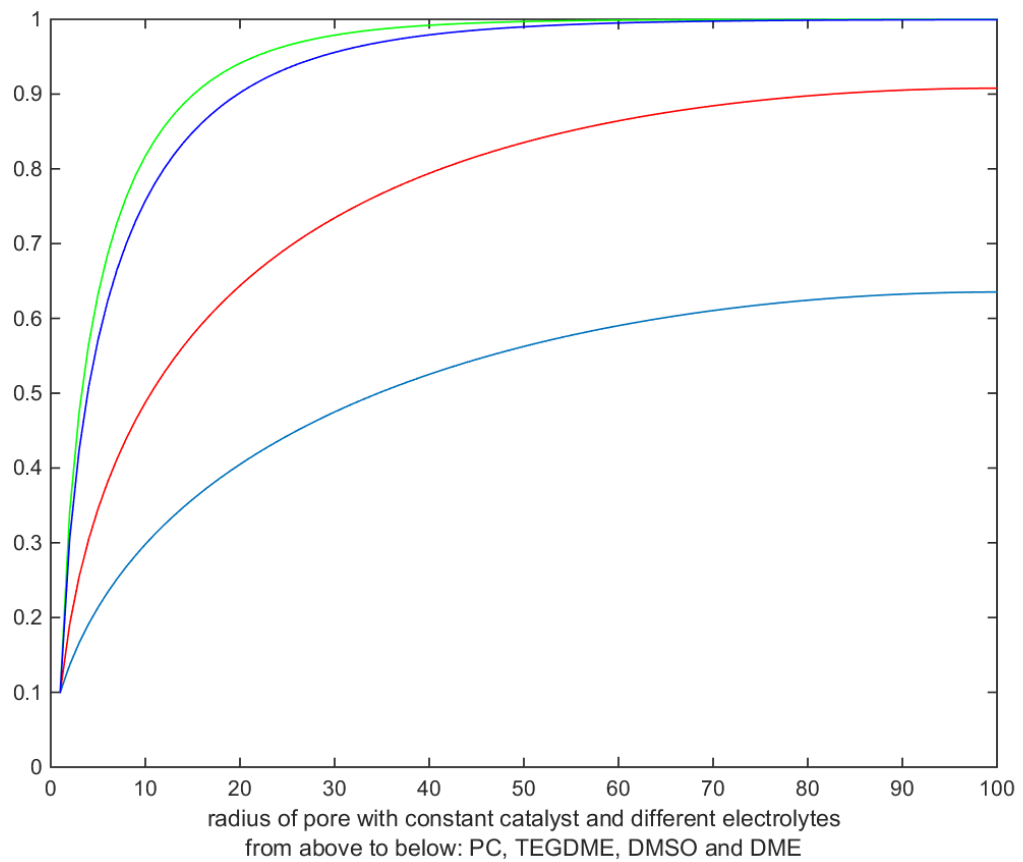

Figure 1.4: The radius of pore with different electrolytes before pore clogging (the radius reaches to 0.1). $\mathrm{Li}_{2} \mathrm{O}_{2}$ is deposited in here in the upper side.
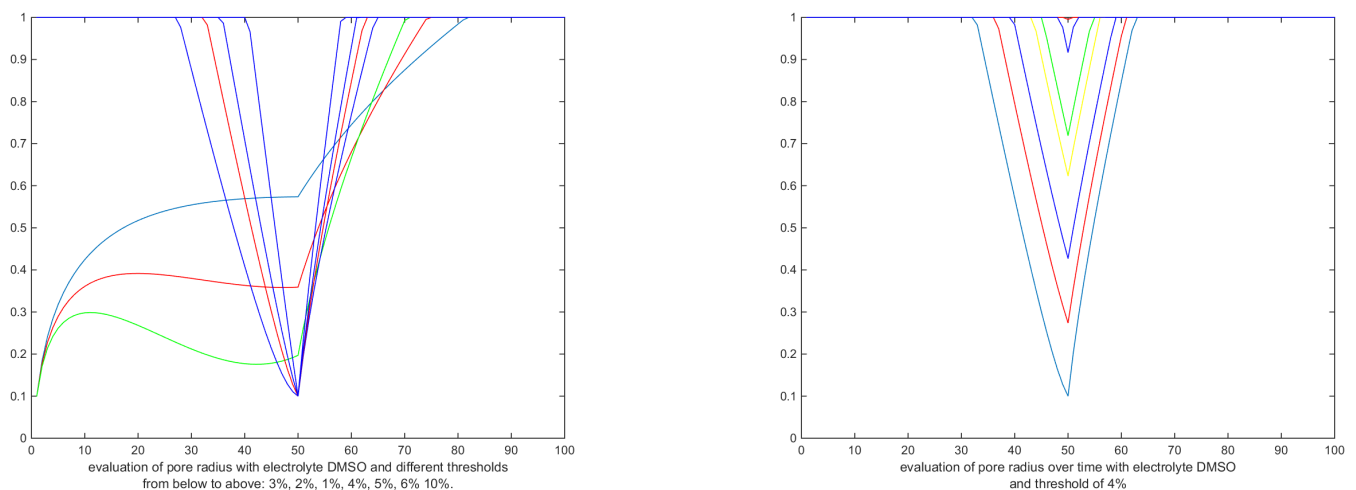

Figure 1.5: The growth profile of $\mathrm{Li}_{2} \mathrm{O}_{2}$ with centered-catalyst positioning, refered from [8] 

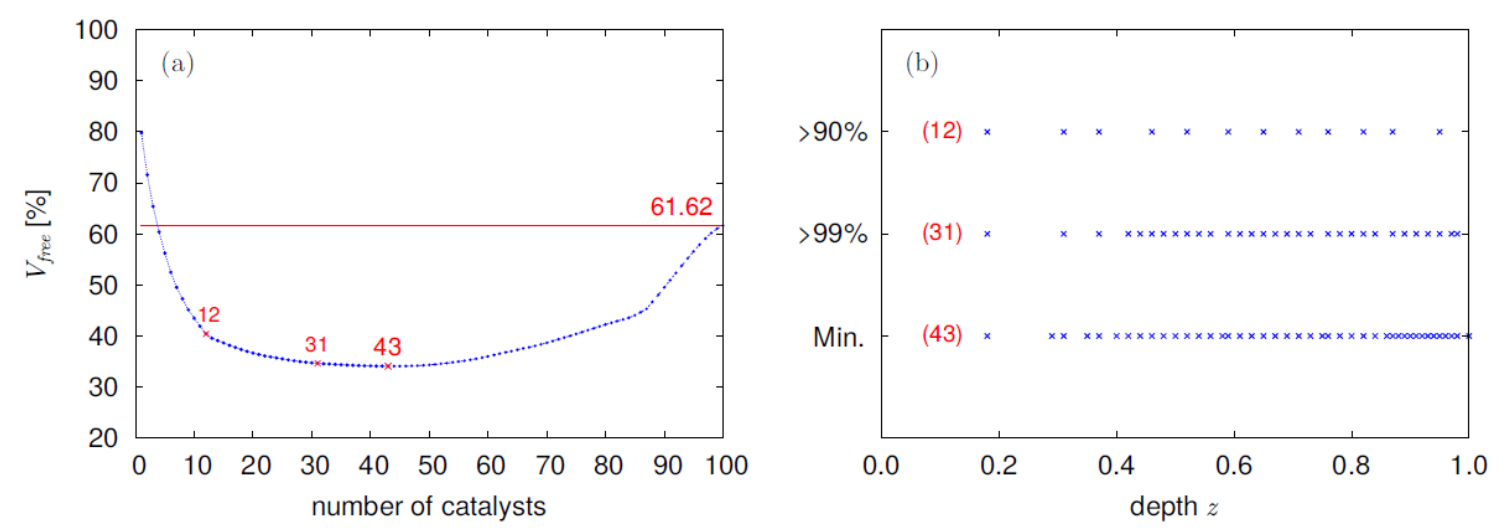

Figure 1.6: (a) Evolution of the minimal free volume after pore clogging as a function of the number of placed catalysts along the pore axis in $D M S O^{L i^{+}}$for a threshold of $6 \%$. The red line indicates the minimal free volume obtained with the continuous growth model. The numbers of catalysts corresponding to $90 \%, 99 \%, 100 \%$ of the final occupied volume are marked in red. (b) Optimal distributions of catalysts along the pore axis corresponding to the three different cases marked in (a). This Figure is refered from [8]

The numerical implementation in [8] is based on the Finite Difference Method (FDM). The greedy method stops when it reaches the first local solution of catalyst function. We would like to present a slightly better optimal solution, the more "global" one by checking the whole iterative steps. This result will be described in details in Chapter 5. Besides, we also suppose Finite Element Method (FEM) to approximate the system of partial differential equations with initial and mixed boundary conditions describing the charging process. All the related analysis and comparisons will be also handed out in this chapter.

Our main interest in this work is to approach to the catalyst positioning problem by proposing a smooth propagation process with a continuous catalytic model. For the optimization problem, we will deal with by two approaches: the sensitivity method and the adjoint method, which are presented in chapter 4 . We refer the readers the next section of Mathematical setting for more understanding on the mathematical features of the problem.

\subsection{Mathematical settings}

The mathematical model for Lithium/ion batteries and its simulation we refer the readers to the series of papers [28], [27], [13] from Ohlberger M. et al. In this work, as mentioned above, we focus only on the mathematical setting for Lithium/air batteries. According to [8] and [32], during the oxygen reduction reaction process, the concentration of oxygen inside the pore drops dast from the oxygen entering side into the other side. As a result, the solid product $\mathrm{Li}_{2} \mathrm{O}_{2}$ is generated and as in the non-aqueous electrolyte environment, Lithium-dioxide is deposited in the surface of the cathode 
pore, which is the reason of the decrease of free volume. With time the pore clogging takes place and limits the oxygen starvation along the pore, $\mathrm{Li}_{2} \mathrm{O}_{2}$-generated reaction could not be occurred any more and the discharge process has to be stop. This circumstance happened due to the low oxygen solunility and diffusivity in non-aqueous electrolytes. Some of theoritical and experimental research had been carried out on this passivation of the cathode of Lithium/air batteries [30], [40]. The mathematical model of this process is presented firstly by Sandhu et al. [32] with the diffusion limited conditions, and later Dabrowski et al. in [8] propose a mathematical description that is based on the latter Sandhu's model and the finite-sized electrolyte phase of Andrei et al. in [3]. The numerical experiments in Chapter 5 will show that the two continuous (catalytic constant) models have the familiar behaviour. In this section, we would like to set up the non-constant continuous catalytic models, in order to have a more natural view in this catalyst positioning problem for Lithium-Air batteries. First, we present the two constant continuous catalytic model based on [32] and [8].

\subsubsection{Mathematical setting of the diffusion-limited model for $\mathrm{Li} /$ air batteries}

In [32] and [8] the authors have been considering the model of $n$ cylindrical pores of radius $r_{p}$, the porous cathode of volume $V_{\text {cath }}$, length $l$, and porosity $\epsilon$, and tortuosity $\tau$. If $r_{p}<<l$, we can assume that the oxygen concentration $c_{O_{2}}$ is angularly symmetric about the diffusion direction or the radial direction along the pore. Thus, the mathematical model is described by dimensionless pore radius, oxygen concentratrion and distance along the pore coordinate increasing toward to the interior side of the Cathode. Both of mathematical models ( [32] and [8]) lead to systems of partial differential equations. The first equation is a reaction - diffusion equation based on Fickian diffusion in a pourous structure without convection [19].

$$
\partial_{t_{p}}\left(\epsilon c_{O_{2}}\right)=\partial_{x_{p}}\left(D_{O_{2}}^{\mathrm{eff}} \partial_{x_{p}} c_{O_{2}}\right)+v_{R}\left(C_{O_{2}}, \epsilon, r_{p}\right)
$$

Here, $D_{O_{2}}^{\text {eff }}$ is the effective diffusion coefficient, which considers the impact of porosity $\epsilon$ and tortuosity $\tau$ on the oxygen mass diffusivity $D_{O_{2}}$ via $D_{O_{2}}^{\text {eff }}=D_{O_{2}} \epsilon / \tau$ or the rate of $L_{2} \mathrm{O}_{2}$ along the active sites located at the pore surfaces per unit cathode.

$v_{R}\left(C_{O_{2}}, \epsilon, r_{p}\right)$ represents the rate of $\mathrm{Li}_{2} \mathrm{O}_{2}$ formation per unit of cathode volume (conversion rate, e.g. [8]).It is written by

$$
v_{R}\left(C_{O_{2}}, \epsilon, r_{p}\right)=-\frac{2 \epsilon}{r_{p}} k C_{O_{2}},
$$

where $k$ is the reaction rate constant per unit pore.

The rate of decrease of pore volume per unit of porous cathode caused by the deposition of $\mathrm{Li}_{2} \mathrm{O}_{2}$ along the active site of pore surface is given by

$$
\partial_{t_{p}} \epsilon=v_{R}\left(C_{O_{2}}, \epsilon, r_{p}\right) \frac{M_{L_{2} O_{2}}}{\rho_{L_{2} O_{2}}}
$$

Tthe mathematical model of Sandhu et al [32] assumes the constant tortuosity $\tau=\tau_{0}=\left(\epsilon_{0}\right)^{\frac{1}{2}}$ with porosity $\epsilon=\epsilon_{0}$ independent of time. Meanwhile, Dabrowski et al. [8] use the Bruggeman relation 


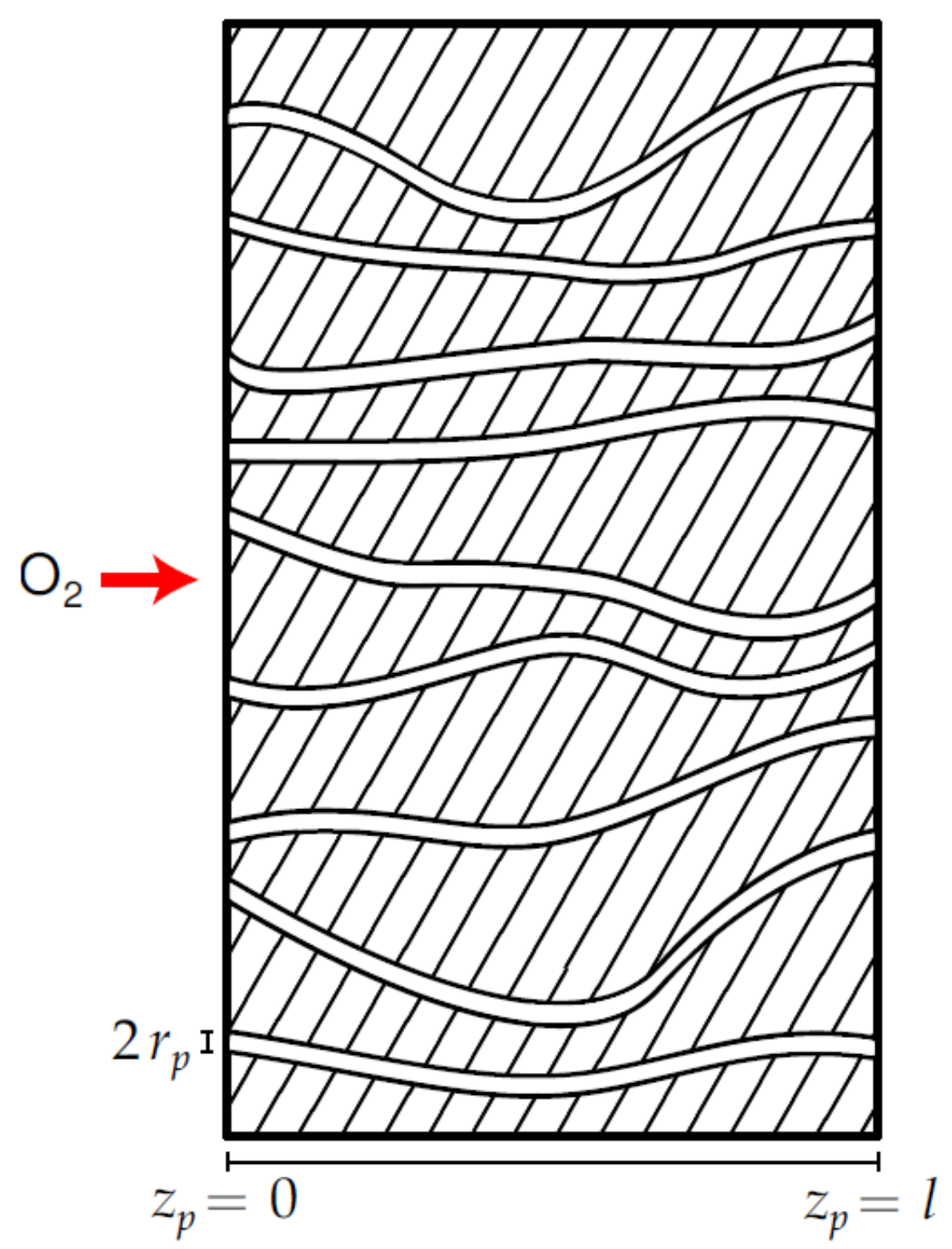

Figure 1.7: Schematic representation of the modeled cathode geometry with porosity $\epsilon$ and tortuosity $\tau$ adapted from [8] 
between tortuosity $\tau$ and porosity $\epsilon$ by $\tau=\epsilon^{-\frac{1}{2}}$ [4].From the Bruggman formula and the initial values of the pore radius, tortuosity and porosity, in conclusion, the porosity and the pore radius are related to each other by

$$
\frac{\epsilon}{\epsilon_{0}}=\left(\frac{r_{p}}{r_{p}^{0}}\right)^{2}
$$

in the first mathematical model ( [32]), and

$$
\left(\frac{\epsilon}{\epsilon_{0}}\right)^{\frac{3}{2}}=\left(\frac{r_{p}}{r_{p}^{0}}\right)^{2}
$$

in the second formulation [8], that lead to the two final normalized reaction-diffusion equations as partial differential equations:

$$
\frac{\partial}{\partial t}\left(r^{\frac{4}{3}} c\right)=\frac{\partial}{\partial x}\left(r^{2} \frac{\partial c}{\partial x}\right)-\beta(t, r, c) r^{\frac{1}{3}} c,(t, x) \in Q_{T}
$$

and

$$
\frac{\partial}{\partial t}\left(r^{2} c\right)=\frac{\partial}{\partial x}\left(r^{2} \frac{\partial c}{\partial x}\right)-\beta(t, r, c) r c,(t, x) \in Q_{T}
$$

The dimensionless quantities are introduced for the equation (1.9) and (1.10) as: $t=\frac{t_{p} D_{O_{2}}}{\tau l^{2}}, t=$ $\frac{t_{p} D_{O_{2}} \sqrt{\epsilon_{0}}}{l^{2}}$, and $r=\frac{r_{p}}{r_{p}^{0}}, x=\frac{x_{p}}{l}, c=\frac{c_{O_{2}}}{c_{O_{2}, 0}}, 0 \leq r, c, x \leq 1,0<t<T$.

Denoting $Q_{T}=[0,1] \times[0, T]$ and $\Omega=[0,1]$, each of equations (1.9), (1.10) accompanied with the equation of decreasing rate of pore volume

$$
\frac{\partial r}{\partial t}=-\gamma(t, r, c) c,(t, x) \in Q_{T}
$$

and the initial conditions:

$$
\begin{gathered}
r(0, x)=r_{0}(x)=1, x \in \Omega, \\
c(0, x)=h(x),
\end{gathered}
$$

and mixed boundary conditions:

$$
\begin{gathered}
c(t, 0)=1, t \in[0, T], \\
c_{x}(t, 1)=0,
\end{gathered}
$$

where $r=r(t, x), c=c(t, x)$ are the pore radius and oxygen concentration inside the pore, whose width is normalized by $1(\mathrm{~cm})$. 
The parameter $\gamma$ and $\beta$ are given by

$$
\begin{array}{r}
\beta=\frac{2 k \tau l^{2}}{D_{O_{2}} r_{p}^{0}}, \\
\gamma=\frac{k c_{O_{2}} M_{L_{i_{2} O_{2}} / \rho_{L_{2} O_{2}}}}{D_{O-2} r_{p}^{0} / \tau l^{2}},
\end{array}
$$

where

$$
k=\frac{i_{\text {geom }} r_{p}^{0}}{4 F C_{O_{2}} \epsilon_{0} l \int_{0}^{1} r c d x},
$$

for the first case and

$$
\begin{array}{r}
\beta=\frac{2 k \tau l^{2}}{D_{O_{2}} \sqrt{\epsilon_{0}} r_{p}^{0}}, \\
\gamma=\frac{3 l_{c}^{2} k_{s} c_{O_{2}} M_{L i_{2} O_{2}} / \rho_{L i_{2} O_{2}}}{2 D_{O_{2}} \sqrt{\epsilon_{0}} r_{p}^{0}},
\end{array}
$$

where the oxygen redution rate $k$ is calculated according to Faraday'laws for the current density change per unit of cathode length $d x_{p}$. For more detail we refer the readers to the references [32] and [8].

$$
k=\frac{i_{\text {geom }} \bar{r}_{p}^{0}}{4 F C_{O_{2}} \epsilon_{0} l \int_{0}^{1} r^{\frac{1}{3}} c d x} .
$$

Now we summariye the above constant catalyst positioning models.

Definition 1.1 (Problems I and II) Problem I: The first mathematical model of [32] as follows

$$
\begin{array}{r}
\frac{\partial}{\partial t}\left(r^{2} c\right)=\frac{\partial}{\partial x}\left(r^{2} \frac{\partial c}{\partial x}\right)-\beta(t, r, c) r c,(t, x) \in Q_{T}, \\
\frac{\partial r}{\partial t}=-\gamma(t, r, c) c,(t, x) \in Q_{T} \\
r(0, x)=r_{0}(x)=1, x \in \Omega, \\
c(0, x)=h(x), \\
c(t, 0)=1, t \in[0, T], \\
c_{x}(t, 1)=0,
\end{array}
$$

where $\beta$ and $\gamma$ are given by equations (1.16) and (1.17) problem I.

Problem II: The second mathematical model of [8]

$$
\frac{\partial}{\partial t}\left(r^{\frac{4}{3}} c\right)=\frac{\partial}{\partial x}\left(r^{2} \frac{\partial c}{\partial x}\right)-\beta(t, r, c) r^{\frac{1}{3}} c,(t, x) \in Q_{T},
$$




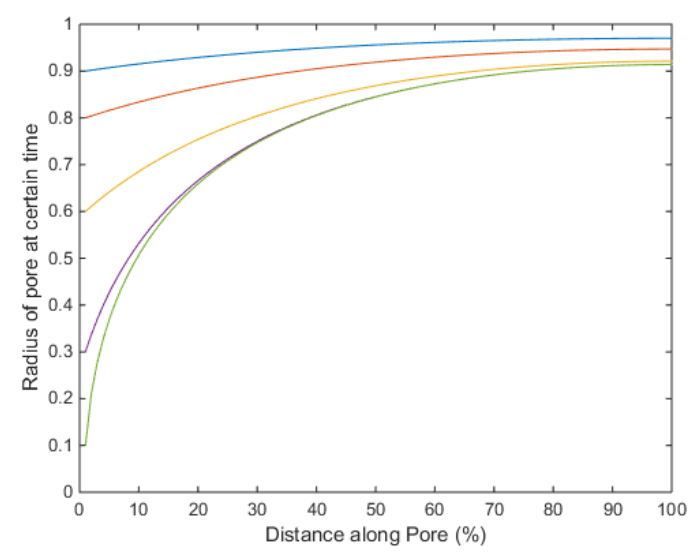

Figure 1.8: The radius of pore at certain times before pore clogging.

$$
\begin{array}{r}
\frac{\partial r}{\partial t}=-\gamma(t, r, c) c,(t, x) \in Q_{T} \\
r(0, x)=r_{0}(x)=1, x \in \Omega, \\
c(0, x)=h(x), \\
c(t, 0)=1, t \in[0, T], \\
c_{x}(t, 1)=0,
\end{array}
$$

with $\beta$ and $\gamma$ defined by equations (1.18) and (1.19).

For the parameters concerned are in the both Problems (Problem I and problems II) we refer to [32] and [8]:

\begin{tabular}{cc}
\hline Parameters & \\
\hline$r_{p, 0}[\mathrm{~cm}]$ & $2.00 .10^{-7}$ \\
$l_{c}[\mathrm{~cm}]$ & 0.07 \\
$\epsilon_{0}$ & 0.73 \\
$M_{\mathrm{Li}_{2} \mathrm{O}_{2}}\left[\frac{\mathrm{g}}{\mathrm{mol}}\right]$ & 45.8765 \\
$r h \mathrm{Li}_{2} \mathrm{O}_{2}\left[\frac{g}{\mathrm{~cm}^{3}}\right]$ & 2.3 \\
$i_{\text {geom }}\left[\frac{\mathrm{mA}}{\mathrm{cm}^{2}}\right]$ & 0.1 \\
\hline
\end{tabular}

In the next Chapters we mainly investigate Problem I. A short formulation of Problem II is considered in Section 3.8. The results can be obtained by similar techniques for Problem II as the analysis features of the two parabolic equations in the two problems has similarities. We refer the readers to the mathematical setting in Chapter 2 and Chapter 3 for details.

The volume of the discharged product $\mathrm{Li}_{2} \mathrm{O}_{2}$ increases during discharge process and the pore is clogged rapidly as demonstrated in the Figure 1.1. The idea of the Greedy algorithm for the discrete catalyst positioning model by Dabrowski et al. in [8] is based one the step-by-step choice of a consequent distribution that is determined on the minimizing progress of an objective functional (the 
free volume left in the pore after the clogging deposition of $\mathrm{Li}_{2} \mathrm{O}_{2}$ takes place). The main strategy of the Greedy algorithm is to to restrict a global optimization to many local optimization problems. Although the Greedy algorithm is a very efficient approach for this optimization problem on the discrete catalyst positioning and for comparing the effect among different electrolyte, it has some following restrictions:

(i) The Greedy method is a forward-optimized method that depends on choosing the local minimizations after every single nodal Greedy step, therefore the running time can take very long (several weeks with $\mathrm{C}++$ ).

(ii) The model that this method relies on is the discrete (catalytic function get number 0 where there is no catalyst, number 1 where catalyst is positioned), while positioning catalyst should be expressed more geometrically by a positive real function, with different positive values at the sites along the pore. The higher values of the catalytic function at the sites where catalyst amount is more, and the lower values for the less active sites along the pore.

(iii) The fast-gradient-typed methods can be applied only for a more continuous catalyst positioning models.

Thus, our idea is to set some model of continuous catalyst positioning characterizing the active catalytic sites along the pore, to investigate the mathematical perspective of the forward problem as well as the related optimization problems and to implement them numerically. Hence, we can give some idea about the optimal geometrical positioning of catalyst to maximize the capacity of the batteries.

We will present the two ways of constructing a catalytic model on Problem I and II as follows

(a) We firstly suppose that $\operatorname{cat}(x)$ is catalyst positioning from the initial time. The value of $\operatorname{cat}(x)$ shows us the active sites effected by catalyst sited along the pore. The growth profile of the discharged product during the ORR process inside the pore is expressed by the model in the problem $I$ or $I I$ with slight adjustment by putting $\operatorname{cat}(x)$ to the right hand side of the equation $(1.20 \mathrm{~b})$ or $(1.21 \mathrm{~b})$ (the equations considering the decrease of free pore volume with respect to the deposition of $\mathrm{Li}_{2} \mathrm{O}_{2}$ in the surface of the pore). This catalytic function does not change with time, it takes the values from 0 to 1 .

$$
\frac{\partial r}{\partial t}=-\gamma(t, r, c) \operatorname{cat}(x) c,(t, x) \in Q_{T}
$$

(b) Learning from the growth kinetics in the nature like bacteria growth and the simple propagation from [8], we propose a time-dependent catalytic function, whose effect is that, once the growth of the solid $\mathrm{Li}_{2} \mathrm{O}_{2}$ reaches to a certain threshold value, this growing trend is propagated to the neighbourhood sites. The function $\operatorname{cat}(x, t)$ is written by the following partial differential equation

$$
\frac{\partial r}{\partial t}=-\gamma(t, r, c) \operatorname{cat}(t, x) c,(t, x) \in Q_{T}
$$


where $\operatorname{cat}(t, x)$ satisfies

$$
\left.\operatorname{cat}_{t}(t, x)=\alpha_{0} \int_{0}^{1} H_{R_{0}}(r(c a t ; y, t)) G(x-y)\right) d y
$$

and

$$
\operatorname{cat}(0, x)=\operatorname{cat}_{0}(x),
$$

where $\alpha_{0}$ is some small positive constant, $R_{0}$ is a positive constant, $0<R_{0}<1$. The Huber function $H_{R_{0}}(r)$ and Gaussian distribution $G(x-y)$ :

$$
G(x-y)=\frac{1}{2 \pi} \exp \left(-\frac{1}{2}(x-y)^{2}\right)
$$

are presented more in details in Appendix A.2 and A.3.

Definition 1.2 (Problems $I_{a}, I_{b}$ and $I I_{a}, I I_{b}$ )

Problem $I_{a}$ : The first mathematical model of [32] in the cat( $x$ ) catalyst positioning model as follows

$$
\begin{array}{r}
\frac{\partial}{\partial t}\left(r^{2} c\right)=\frac{\partial}{\partial x}\left(r^{2} \frac{\partial c}{\partial x}\right)-\beta(t, r, c) r c,(t, x) \in Q_{T}, \\
\frac{\partial r}{\partial t}=-\gamma(t, r, c) \operatorname{cat}(x) c,(t, x) \in Q_{T}, \\
r(0, x)=r_{0}(x)=1, x \in \Omega, \\
c(0, x)=h(x), \\
c(t, 0)=1, t \in[0, T], \\
c_{x}(t, 1)=0,
\end{array}
$$

where $\beta$ and $\gamma$ are given by equations (1.16) and (1.17).

Problem $I_{b}$ : The first mathematical model of [32] in the cat $(t, x)$ catalyst positioning model as follows

$$
\begin{array}{r}
\frac{\partial}{\partial t}\left(r^{2} c\right)=\frac{\partial}{\partial x}\left(r^{2} \frac{\partial c}{\partial x}\right)-\beta(t, r, c) r c,(t, x) \in Q_{T}, \\
\frac{\partial r}{\partial t}=-\gamma(t, r, c) \operatorname{cat}(t, x) c,(t, x) \in Q_{T}, \\
r(0, x)=r_{0}(x)=1, x \in \Omega, \\
c(0, x)=h(x), \\
c(t, 0)=1, t \in[0, T], \\
c_{x}(t, 1)=0, \\
\left.\operatorname{cat}_{t}(t, x)=\alpha_{0} \int_{0}^{1} H_{R_{0}}(r(c a t ; y, t)) G(x-y)\right) d y, \operatorname{cat}(0, x)=\operatorname{cat}_{0}(x),
\end{array}
$$


with $\beta$ and $\gamma$ defined by equations (1.18) and (1.19).

Problem II $I_{a}$ : The first mathematical model of [8] in the cat $(t, x)$ catalyst positioning model as follows

$$
\begin{array}{r}
\frac{\partial}{\partial t}\left(r^{\frac{4}{3}} c\right)=\frac{\partial}{\partial x}\left(r^{2} \frac{\partial c}{\partial x}\right)-\beta(t, r, c) r^{\frac{1}{3}} c,(t, x) \in Q_{T}, \\
\frac{\partial r}{\partial t}=-\gamma(t, r, c) \operatorname{cat}(x) c,(t, x) \in Q_{T}, \\
r(0, x)=r_{0}(x)=1, x \in \Omega, \\
c(0, x)=h(x), \\
c(t, 0)=1, t \in[0, T], \\
c_{x}(t, 1)=0,
\end{array}
$$

where $\beta$ and $\gamma$ are given by equations (1.16) and (1.17).

Problem II $I_{b}$ : The first mathematical model of [8] in the cat $(t, x)$ catalyst positioning model as follows

$$
\begin{array}{r}
\frac{\partial}{\partial t}\left(r^{\frac{4}{3}} c\right)=\frac{\partial}{\partial x}\left(r^{2} \frac{\partial c}{\partial x}\right)-\beta(t, r, c) r^{\frac{1}{3}} c,(t, x) \in Q_{T}, \\
\frac{\partial r}{\partial t}=-\gamma(t, r, c) \operatorname{cat}(t, x) c,(t, x) \in Q_{T}, \\
r(0, x)=r_{0}(x)=1, x \in \Omega, \\
c(0, x)=h(x), \\
c(t, 0)=1, t \in[0, T], \\
c_{x}(t, 1)=0, \\
\left.\operatorname{cat}_{t}(t, x)=\alpha_{0} \int_{0}^{1} H_{R_{0}}(r(c a t ; y, t)) G(x-y)\right) d y, \operatorname{cat}(0, x)=\operatorname{cat}_{0}(x),
\end{array}
$$

with $\beta$ and $\gamma$ defined by equations (1.18) and (1.19).

\subsubsection{Optimization Problems on catalyst positioning models}

We herein would like to formulate the optimization problem of this catalyst positioning model.

\subsubsection{The first optimization problem}

We now aim at setting the optimization problems in order to maximize the volume of discharged products deposited before pore clogging. The first approach, we consider the minimization problem 
of remaining pore volume with $T, 0<T<\infty$ is the some final time.

For Problem $I_{a}$ and Problem $I I_{a}$, the free volume $V(c a t)$ of the pore after some time T is defined by:

$$
V(c a t)=\int_{0}^{1} r^{2}(c a t ; T, x) d x,
$$

For Problem $I_{b}$ and Problem $I I_{b}$, the free volume $V\left(c a t_{0}\right)$ of the pore after some time T is defined by:

$$
V\left(c a t_{0}\right)=\int_{0}^{1} r^{2}\left(c a t_{0} ; T, x\right) d x
$$

We give the definition of the first optimization problem as follows:

Definition 1.3 (i) The first optimization problem is for Problem $I_{a}$ (alternatively for Problem $I I_{a}$ )

$$
\min _{c a t \in A} V(\text { cat }),
$$

where $\boldsymbol{A}$ is some set of catalytic function cat $(x)$, subject to Problem $I_{a}$ (or Problem $I I_{a}$ alternatively).

(ii) The first optimization problem is for Problem $I_{b}$ (alternatively for Problem $I_{b}$ )

$$
\min _{\text {cat } \boldsymbol{A}_{\text {cat }}} V\left(\text { cat }_{0}\right)
$$

where $\boldsymbol{A}_{\text {cat }_{0}}$ is some set of catalytic function at the initial time cat $(0, x)=$ cat $_{0}(x)$, subject to Problem $I_{b}$ (or Problem $I_{b}$ alternatively).

The two problem (1.33) and (1.34) will be solved by dealing with the sensitivity systems and adjoint systems in chapter 4.

\subsubsection{The second optimization problems}

For the convenience to apply the adjoint method that we will present also in the Chapter 4, the second optimization problem, that minimizes the mean value of free volume in the pore in a small interval of time $\left[T_{1}, T\right]$. Therefore,

for Problem $I_{a}$ and Problem $I I_{a}$, we wish to minimize

$$
\mathcal{V}(\text { cat })=\int_{T_{1}}^{T} \int_{0}^{1} r^{2}(c a t ; t, x) d x d t,
$$

for Problem $I_{b}$ and Problem $I I_{b}$, the objective functional is defined by

$$
\mathcal{V}\left(\text { cat }_{0}\right)=\int_{T_{1}}^{T} \int_{0}^{1} r^{2}\left(c a t_{0} ; t, x\right) d x d t
$$

We give the definition of the first optimization problem as follows. 
Definition 1.4 (i) The first optimization problem is for Problem $I_{a}$ (alternatively for Problem $I_{a}$ )

$$
\min _{\text {cat } \in \boldsymbol{A}} \mathcal{V}(\text { cat })
$$

where $\boldsymbol{A}$ is some set of catalytic function cat $(x)$, subject to Problem $I_{a}$ (or Problem $I I_{a}$ alternatively).

(ii) The first optimization problem is for Problem $I_{b}$ (alternatively for Problem $I I_{b}$ )

$$
\min _{c a t \in \boldsymbol{A}_{\text {cat }}} \mathcal{V}\left(\text { cat }_{0}\right),
$$

where $\boldsymbol{A}_{\text {cat }}$ is some set of catalytic function at the initial time cat $(0, x)=$ cat $_{0}(x)$, subject to Problem $I_{b}$ (or Problem $I_{b}$ alternatively).

\subsection{The main results of the Thesis}

In the Chapter Introduction we present an overview on the subject of the Thesis and then the mathematical form with two "soft" propagation models of the catalyst problem and formulate the optimization problems on those models.

In the Chapter 1, we summarize some basic notions and results needed to the next Chapters.

In the chapter 2, the well-posedness of the forward problem is proved with choosing the appropriate functional spaces of the related parameters and variables. The Fréchet differentiability of the pore radius and the oxygen concentration with respect to catalytic function is also established in the related functional spaces.

In the chapter 3, the existence of the solution of optimization problems is considered by mean of sensitivity method and adjoint method. The ways to solve those optimization problems are shown therein.

In chapter 4, we solve numerically the optimization problem. Some methods are carried out to calculate the Frechet gradient in order to minimize optimal functional. We also propose some potential local optimal solutions of catalytic functions to the optimization problem.

For the sake of clarity for readers we make the following tables to indicates the proofs for the PDEs models: The existence, uniqueness of the solutions, the differentiability of parameter-to-state map $r_{c a t}$ or $r_{c a t_{0}}$, the solvability of the two optimization methods and the numerical implementations:

(i) The table 1.1 illustrates the existence, uniqueness of the solutions and the differentiability of parameter-to-state map for Problem $I_{a}$ and $I_{b}$. In the chapter 3, Sections 3.1-3.2, we prove the well-posedness of the Problem $I_{a}$ and the Frechet differentiability of radius $r$ with respect to catalytic function $\operatorname{cat}(x)$ in Section 3.4. In the Sections 3.6-3.7, we state the proofs of the wellposedness and the Frechet differentiability of radius $r$ with respect to catalytic function $\operatorname{cat}(x)$ of the Problem $I_{b}$. The proofs for the Problem $I I, I I_{a}, I I_{b}$ are supposed to achieve analogously. 
Table 1.1: The well-posedness of solution and the differentiability of the parameter-to-state $r_{c a t}$ or $r_{c a t_{0}}$ for Problem $I_{a}$ and $I_{b}$.

\begin{tabular}{|l|l|l|}
\hline & $I_{a}$ & $I_{b}$ \\
\hline The well-posedness & Sections 3.1-3.2 & Section 3.6 \\
\hline The differentiability of $r_{c a t}$ & Section 3.4 & Section 3.7 \\
\hline
\end{tabular}

(ii) The two optimization problems for the Problem $I_{a}$ and $I_{b}$ are considered in Chapter 4 and demonstrated in the table 1.2. We apply the sensitivity method for the first optimization problem and the adjoint method for the second optimization problem.

Table 1.2: The two optimization problems $I_{a}$ and $I_{b}$

\begin{tabular}{|l|l|l|}
\hline & $I_{a}$ & $I_{b}$ \\
\hline The first optimization problem using sensitivity method & Section 4.2 & Section 4.2 \\
\hline The second optimization problem using adjoint method & Subsection 4.2.1 & Subsection 4.2.2 \\
\hline
\end{tabular}

(iii) In the Chapter 5 we consider the implementation for two optimization problems for the Problem $I_{a}$, that is expressed in the table 1.3 .

Table 1.3: Numerical implementation for optimization problems

\begin{tabular}{|l|l|}
\hline & $I_{a}$ \\
\hline The first optimization problem & Subsection 5.4.1 \\
\hline The second optimization problem & Subsection 5.4.2 \\
\hline
\end{tabular}





\section{Chapter 2}

\section{Mathematical preliminaries}

This Chapter is devoted to provide some basic notions in Functional Analysis needed for linear parabolic equations in Hilbert spaces, and as well as some concepts of optimization theory. We start by giving definitions of Sobolev spaces and Sobolev-Bochner spaces and then parabolic PDEs with mixed boundary conditions, the regularity of its solution, delivery the solvability of the mixed boundary value problems.

\subsection{Elementary concepts for PDEs}

\subsubsection{Sobolev spaces}

Let $\Omega$ be an open, bounded subset of $\mathbb{R}^{n}, n=1,2,3$.

Definition 2.1 (Weak partial direvative, $p .212,[12]$ ) Let $L_{\text {loc }}^{1}(\Omega)$ be the set of locally integrable functions defined on the open set $\Omega$. Suppose $u, v \in L_{\text {loc }}^{1}(\Omega)$, and $\alpha$ is multi-index. We say that $v$ is the $\alpha^{\text {th }}$-weak partial derivative of $u$, written

$$
D^{\alpha} u=v
$$

provided

$$
\int_{\Omega} u D^{\alpha} \varphi d x=(-1)^{|\alpha|} \int_{\Omega} v \varphi d x, \text { for all } \varphi \in C_{c}^{\infty}(\Omega),
$$

where $C_{c}^{\infty}(\Omega)$ denotes the space of infinitely differentiable functions $\varphi: \Omega \rightarrow \mathbb{R}$, with compact support in $\Omega$. Here, $D^{\alpha} \varphi$ is defined as

$$
D^{\alpha} \varphi=\frac{\partial^{|\alpha|} \varphi}{\partial x_{1}^{\alpha_{1}} \cdots \partial x_{n}^{\alpha_{n}}}
$$

In this Thesis we need the following standard Sobolev spaces (See [20], [21], [12], [35]) 
Definition 2.2 (Space $H^{1}(\Omega)$ ) The Sobolev space $H^{1}(\Omega)$ is the set of all elements $u(x) \in L^{2}(\Omega)$ having weak (distributional) derivatives $\frac{\partial u}{\partial x_{i}} \in L^{2}(\Omega), i=1, \cdots, n$, with scalar product

$$
(u, v)_{H^{1}(\Omega)}:=\int_{\Omega} u v+\sum_{i=1}^{n} \frac{\partial u}{\partial x_{i}} \frac{\partial v}{\partial x_{i}} d x
$$

Definition 2.3 The Sobolev space $H_{0}^{1}$ is the completion of $C_{0}^{1}(\Omega)$ in the norm of $H^{1}(\Omega)$. In case $\Omega$ is piecewise Lipschitz boundary, we have

$$
H_{0}^{1}(\Omega)=\left\{u \in H^{1}(\Omega),\left.u\right|_{\partial \Omega}=0\right\} .
$$

Space $H^{-1}(\Omega)$ is dual space to space $H_{0}^{1}(\Omega)$. Space $\left(H^{1}(\Omega)\right)^{\prime}$ is dual space to space $H^{1}(\Omega)$.

Definition 2.4 For $f \in H^{-1}(\Omega)$ we define the norm

$$
\|f\|_{H^{-1}(\Omega)}=\sup \left\{\langle f, v\rangle \mid v \in H_{0}^{1}(\Omega),\|v\|_{H_{0}^{1}(\Omega)} \leq 1\right\}
$$

The functional space $H^{-1}$ can be characterized as follows [12].

Theorem 2.5 1. Assume that $f \in H^{-1}(\Omega)$. Then there exist functions $f^{0}, f^{1} \in L^{2}(\Omega)$ such that

$$
\langle f, v\rangle=\int_{\Omega}\left(f^{0} v+f^{1} v_{x}\right) d x\left(\forall v \in H_{0}^{1}(\Omega)\right)
$$

2. Furthermore,

$$
\|f\|_{H^{-1}(\Omega)}=\inf \left\{\left(\int_{\Omega} \sum_{i=0}^{1}\left|f^{i}\right|^{2} d x\right)^{\frac{1}{2}}|f| \text { satisfies }(2.4) \text { forf } f^{0}, f^{1} \in L^{2}(\Omega)\right\}
$$

\subsubsection{Spaces involving time}

To study the evolution equations, we need spaces involving time(section 23.5 in [38], Chapter 4 [37], [22] and chapter 5 in [12]).

Let $X$ be a Banach space with norm $\|\cdot\|_{X}$.

Definition 2.6 P.285 [12] The space $L^{p}(0, T ; X)$ consists of all measurable functions $u:[0, T] \longrightarrow X$ with

1.

$$
\|u\|_{L^{p}(0, T ; X)}:=\left(\int_{0}^{T}\|u(t)\|_{X}^{p} d t\right)^{\frac{1}{p}}<\infty
$$

for $1 \leq p \leq \infty$, and 
2.

$$
\|u\|_{L^{\infty}(0, T ; X)}:=\operatorname{esssup}_{0 \leq t \leq T}\|u(t)\|_{X}<\infty .
$$

Definition 2.7 P.285 [12] The space $C(0, T ; X)$ comprises all continuous functions $u:[0, T] \longrightarrow X$ with

$$
\|u\|_{C(0, T ; X)}:=\max _{0 \leq t \leq T}\|u\| \leq \infty .
$$

A basic definition of studying evolution equations is the definition of generalized (weak) derivatives in time.

Definition 2.8 (Weak time derivative, P.285 [12]) Let $u \in L^{1}(0, T ; X)$. we say $v \in L^{1}(0, T ; X)$ is the weak derivative of of order $n$ of $u$, written

$$
u^{(n)}=v,
$$

provided

$$
\int_{0}^{T} \phi^{(n)}(t) u(t) d t=(-1)^{n} \int_{0}^{T} \phi(t) v(t) d t
$$

for all scaler test functions $\phi:(0, T) \rightarrow \mathbb{R}, \phi \in C_{c}^{\infty}(0, T)$.

This is a direct generalization of the notion of the weak derivative of real-valued functions. The integrals in (2.5) are vector-valued Lebesgue integrals (or Bochner integrals), which are defined by the Lebesgue integral of an integrable real-valued function as the $L^{1}$-limit of integrals of simple functions. See [Wloka] for further understanding such integrals and the weak derivative of vector-valued functions.

Proposition 2.9 (Existence of $u^{(n)}$ ) P.120 [38] Assume that $V \subset H \subset V^{*}$ is an evolution triple, and let $1 \leq p, q \leq \infty, 0<T<\infty$. Then the following statements are valid

(i) Uniqueness. For $u \in L_{p}(0, T ; V)$, the weak derivative $u^{(n)}$ is unique as an element of $L_{q}\left(0, T ; V^{*}\right)$.

(ii) Existence. Let $u \in L_{p}(0, T ; V)$. Then there exists the weak derivative $u^{(n)} \in L_{q}\left(0, T ; V^{*}\right)$ if there is a function $w \in L_{q}\left(0, T ; V^{*}\right)$ such that

$$
\int_{0}^{T}(u(t) \mid v)_{H} \varphi^{(n)}(t) d t=(-1)^{n} \int_{0}^{T}<w(t), v>_{V} \varphi(t) d t
$$

Then $u^{(n)}=w$ and

$$
\frac{d^{n}}{d t^{n}}(u(t) \mid v)_{H}=<u^{(n)}(t), v>_{V}
$$

holds for all $v \in V$ and almost all $t \in[0, T]$. Here, $\frac{d^{n}}{d t^{n}}$ denoted by the $n$-th weak derivative of real functions on $[0, T]$. 
Proof. We refer Zeidler [38], proposition 23.20.

Proposition 2.10 (The space $W_{p}^{1}(0, T ; V, H)$ ). Let $V \subset H \subset V^{*}$ is an evolution triple, and let $1<p<$ $\infty, p^{-1}+q^{-1}=0$ and $0<T<\infty$. Then the following statements hold :

(i) The space $W_{p}^{1}(0, T ; V, H)$. The set of all $u \in L^{p}(0, T ; V)$ that have weak derivatives

$$
u^{\prime} \in L^{q}\left(0, T ; V^{*}\right)
$$

forms a real Banach space with the norm

$$
\|u\|_{W_{p}^{1}}:=\|u\|_{L^{p}(0, T ; V)}+\left\|u^{\prime}\right\|_{L^{q}\left(0, T ; V^{*}\right)}
$$

We will denote this space by $W_{p}^{1}(0, T ; V, H)$

(ii) Regularization. The embedding

$$
W_{p}^{1}(0, T ; V, H) \hookrightarrow C([0, T] ; H)
$$

is continuous.

(iii) Integral by parts. For all $u, v \in W_{p}^{1}(0, T ; V, H)$ and arbitrary $t, s$ with $0 \leq s \leq t \leq T$, the following generalized formula of integration by parts holds:

$$
(u(t) \mid v(t))_{H}-(u(s) \mid v(s))_{H}=\int_{s}^{t}<u^{\prime}(\tau), v(\tau)>_{V} d \tau+<u(\tau), v^{\prime}(\tau)>_{V} d \tau .
$$

Here, the values $u(t), v(t), u(s), v(s)$ are the values of the continuous functions $u, v:[0, T] \rightarrow H$ in sense of (ii).

Proof. We refer Zeidler [38], proposition 23.23 and problem 23.10.

Lemma 2.11 [35] For all $y, p \in W\left(0, T ; H^{1}(\Omega)\right)$ the formula of integration by part holds

$$
\int_{0}^{T}\left(y^{\prime}(t), p(t)\right)_{H_{0}^{-1}(\Omega), H_{0}^{1}(\Omega)} d t=(y(T), p(T))_{L^{2}(\Omega)}-(y(0), p(0))_{L^{2}(\Omega)}-\int_{0}^{T}\left(p^{\prime}(t), y(t)\right)_{H_{0}^{-1}(\Omega), H_{0}^{1}(\Omega)} d t .
$$

Taking $p=y$ in the formula of integration by parts, we find that any $y \in W\left(0, T ; H^{1}(\Omega)\right)$ satisfies the useful identity

$$
\int_{0}^{T}\left(y^{\prime}(t), y(t)\right)_{H_{0}^{-1}(\Omega), H_{0}^{1}(\Omega)} d t:=\frac{1}{2}\|y(T)\|_{L^{2}(\Omega)}^{2}-\frac{1}{2}\|y(0)\|_{L^{2}(\Omega)}^{2} .
$$

Definition 2.12 We also define

$$
W\left(0, T ; H^{1}(\Omega)\right)=\left\{u: u \in L^{2}\left(0, T ; H_{0}^{1}(\Omega)\right), u^{\prime} \in L^{2}\left(0, T ; H^{-1}(\Omega)\right)\right\},
$$

and

$$
W\left(0, T ; H^{1}(\Omega)\right)=\left\{u: u \in L^{2}\left(0, T ; H^{1}(\Omega)\right), u^{\prime} \in L^{2}\left(0, T ;\left(H^{1}(\Omega)\right)^{\prime}\right)\right\}
$$


Lemma 2.13 (Variational lemma). Let $V$ be Banach space. Then it follows from $u \in L^{1}(0, T ; X)$ and

$$
\int_{0}^{T} \varphi(t) u(t) d t=0 \text { for all } \varphi \in C_{c}^{\infty}(0, T)
$$

that $u=0$ in $L^{1}(0, T, X)$, i.e.,

$$
u(t)=0 \text { for almost all } t \in[0, T]
$$

Proof. We refer Zeidler [38], proposition 23.10.

Definition 2.14 Taking $\Omega=(0,1)$, denote $Q_{T}=(0, T] \times \Omega$, we define space:

$$
W^{1, \infty}\left(Q_{T}\right)=\left\{r \in L^{\infty}\left(Q_{T}\right), r_{t} \in L^{\infty}\left(Q_{T}\right), r_{x} \in L^{\infty}\left(Q_{T}\right)\right\},
$$

\subsection{Mixed boundary value problem for one-dimensional linear parabolic equations}

We will use Fixed-point theory to approach our system of PDEs (problem $I$ and $I I$ with different continuous catalyst models for Lithium/air batteries: Problems $\left.I_{a}, I I_{a}, I_{b}, I I_{b}\right)$. Every system consists of a diffusion equation and a decreasing radius equation. By mean of the Fixed-point method, we can consider only the diffusion equation in term of the equation of oxygen concentration $c$ (the radius $r$ is fixed). This diffusion equation leads us to considering an one-dimensional mixed boundary value parabolic equation, which is described as follows:

We assume $\Omega, Q_{T}$ are defined in Definition 2.14 for some fixed time $T>0$. Consider the initial/boundaryvalue problem

$$
\left\{\begin{array}{l}
u_{t}+\mathcal{L} u=f, \text { in } Q_{T} \\
u(t, 0)=0, u_{x}(1)=0, t \in[0, T] \\
u(0, x)=u_{0}, x \in \Omega,
\end{array}\right.
$$

where $f: Q \rightarrow \mathbb{R}$ and $u_{0}: \Omega \rightarrow \mathbb{R}$ are given, and $u=u(t, x)$ is unknown, the operator $\mathcal{L}$ denotes for each $t \in[0, T]$ the second-order non-divergence partial differential operator

$$
\mathcal{L} u=-a(t, x) u_{x x}+b(t, x) u_{x}+c(x, t) u,
$$

for given coefficients $a(t, x), b(t, x), c(t, x)$ and

$$
a(t, x)>\theta>0 \text {, for a.e. }(x, t) \in Q_{T} .
$$

Denote by $V=\left\{v \in H^{1}(\Omega), v(0)=0,\right\}$, and endow it with the scalar product: 


$$
\|v\|_{V}=\int_{0}^{1} u_{x} v_{x} d x
$$

Define the bilinear formula:

$$
B[u, v ; t]:=\int_{\Omega} a(\cdot, t) u_{x} v_{x}+\left(b(\cdot, t)+a_{x}(\cdot, t)\right) u_{x} v+c(\cdot, t) u v d x
$$

for $u, v \in V$ a.e. $0 \leq t \leq T$.

To formulate a definition of a weak solution of the problem (2.6), we formally multiply (2.6) by a test function $v \in V$, integrate the result over $\Omega$, and apply the formula of integration by part, we get

$$
<u^{\prime}(t), v>_{V, V^{\prime}}+B[u(t), v ; t]=(f(t), v)_{L^{2}(\Omega)}, \text { for a.e } 0 \leq t \leq T,
$$

where $B$ is defined by (2.9). In the equation (2.10), we have used the "vector-valued" notion $u(t)=$ $u(\cdot, t)$. Further, in order to ensure that the definition of weak solution makes sense, we need the following assumptions.

\section{Assumption 2.15 Assume that}

(i) the coefficients of B in the formula (2.9) satisfy

$$
a(x, t), a_{x}(x, t), b(x, t), c(x, t) \in L^{\infty}\left(Q_{T}\right) .
$$

(ii) Operator $\frac{\partial}{\partial t}+\mathcal{L}$ is the parabolic operator.

(iii) $f \in L^{2}\left(0, T ;\left(H^{1}(\Omega)\right)^{\prime}\right)$ and $u_{0} \in L^{2}(\Omega)$.

We denote the duality pairing between $H^{1}(\Omega)$ and $\left(H^{1}(\Omega)\right)^{\prime}$ by

$$
<\cdot, \cdot>_{H^{1}(\Omega),\left(H^{1}(\Omega)\right)^{\prime}}: H^{1}(\Omega) \times\left(H^{1}(\Omega)\right)^{\prime} \rightarrow \mathbb{R} .
$$

Now we introduce the definition of the weak solution as follows

Definition 2.16 (Weak solution of the problem (2.6)) We say that a function $u \in W(0, T ; V)$ is a weak solution of the parabolic initial/boundary-value problem (2.6) if

1.

$$
<u^{\prime}, v>+B[u, v ; t]=<f, v>
$$

for each $v \in V$ and for a.e. $t \in[0, T]$,

2. $u(0)=u_{0}$. 
The initial condition $u(0)=u_{0}$ has meaning since $W(0, T ; V) \hookrightarrow C\left(0, T ; L^{2}(\Omega)\right.$ ) (Proposition 2.10, item (ii)).

Since the coefficients of B are uniformly bounded in time, it repeating the proof of Theorem 2, p. 300 [12]. We have the following result:

Lemma 2.17 (Energy estimate). There exist constants $\alpha, \beta>0$ and $\gamma \geq 0$ such that

(i)

$$
|B[u, v]| \leq \alpha\|u\|_{V}\|v\|_{V}
$$

and

(ii)

$$
\beta\|u\|_{V}^{2} \leq B[u, u]+\gamma\|u\|_{L^{2}(\Omega)}^{2}
$$

for all $u, v \in V$.

For further proof, we need also the following Theorem

Theorem 2.18 There exist functions $\omega_{k}=\omega_{k}(x), k=1,2, \cdots$, that are

(i) $\left\{\omega_{k}\right\}_{k=1}^{\infty}$ is an orthogonal basic of $V$.

(ii) $\left\{\omega_{k}\right\}_{k=1}^{\infty}$ is an orthonormal basic of $L^{2}(\Omega)$.

Proof. Following the proof from Theorem 1 (Eigenvalues of symmetric elliptic operators), p.335 [12] with replacing the Dirichlet boundary problem for elliptic equation by the mixed boundary problem for elliptic equations. Finally we choose $\left\{\omega_{k}\right\}_{k=1}^{\infty}$ the eigenfunctions of $L=-\Delta$ in $V$.

The first section of Chapter 7, Evans [12] is devoted to studying the weak solution of parabolic equations of the Dirichlet problem. The existence and regularity of weak solutions are established by the use of $L^{2}$-energy estimates via the Galerkin method. For the regularity results, it is assumed that the coefficients of $L$ are smooth on $\Omega$ and independing of $\mathrm{t}$, which are not suitable for Problem $I_{a}, I_{b}$, $I I_{a}$ and $I I_{b}$. We will then rely basically on the results from Evans [12] to prove the basic results on solving the mixed boundary condition (2.6) such as the existence, uniqueness and regularity of the weak solution.

We shall use the Galerkin method in chapter 7, [12] for studying the system (2.6) by approximating $u$ by

$$
u_{m}(t):=\sum_{k=1}^{m} d_{m}^{k}(t) \omega_{k}
$$

where

$$
d_{m}^{k}(0)=\left(u_{0}, \omega_{k}\right), k=1, \cdots, m
$$


To seek the function $u_{m}$, we project the equation (2.6) onto $V_{m}$, that leads to a systems of ordinary differential equations (ODEs) for $u_{m}$

$$
\left(u_{m}^{\prime}, \omega_{k}\right)+B\left[u_{m}, \omega_{k} ; t\right]=\left(f, \omega_{k}\right),
$$

with $k=1, \cdots, m$ and $t \in[0, T]$. which by mean of the standard existence theory for ODEs has unique absolutely continuous solution. We recall the Theorem 1 (Construction of approximate solution) in p. 354 [12]:

Theorem 2.19 For each integer $m=1,2, \cdots$ there exists a unique function $u_{m}$ of the form (2.13), satisfying (2.14) and (2.15).

Theorem 2.20 There exist a constant $C$, depending on $\Omega, T$ and the coefficient of $B$, such that

$$
\begin{array}{r}
\max _{0 \leq t \leq T}\left\|u_{m}(t)\right\|_{L^{2}(\Omega)}+\left\|u_{m}\right\|_{L^{2}(0, T ; V)}+\left\|u_{m}^{\prime}\right\|_{L^{2}\left(0, T ; V^{\prime}\right)} \leq \\
\leq C\left(\|f\|_{L^{2}\left(0, T ; L^{2}(\Omega)\right)}+\left\|u_{0}\right\|_{L^{2}(\Omega)}\right),
\end{array}
$$

for $m=1,2, \cdots$

Next, we let $m \rightarrow \infty$ to build a weak solution. According to the uniform estimates in (2.16), we see that the uniform bounds of the sequence $\left\{u_{m}\right\}$ in $W(0, T ; V)$ is weakly compact in $W(0, T ; V)$. We get the result about the existence of the weak solution:

Theorem 2.21 Suppose that the assumptions 2.15 are satisfied. Then there exists a weak solution $u \in W(0, T ; V)$ of the equation (2.6), and in fact

$$
u \in L^{2}(0, T ; V) \cap L^{2}\left(0, T ; H^{1}(\Omega)\right) \cap L^{\infty}\left(0, T ; L^{2}(\Omega)\right) .
$$

Reminding that the norm of $V$ and $H^{1}(\Omega)$ are equivalent, we have estimate

$$
\begin{aligned}
\operatorname{ess} \sup _{[0, T]}\|u(t)\|_{L^{2}(\Omega)} & +\|u\|_{L^{2}\left(0, T ; H^{1}(\Omega)\right)}+\left\|u^{\prime}\right\|_{L^{2}\left(0, T ; V^{\prime}\right)} \leq \\
& \leq C\left(\|f\|_{L^{2}\left(0, T ; L^{2}(\Omega)\right)}+\left\|u_{0}\right\|_{H_{0}^{1}(\Omega)}\right) .
\end{aligned}
$$

Theorem 2.22 Suppose that the assumptions 2.15 are satisfied. Then the weak solution of the equation (2.6) is unique.

Theorem 2.23 (Improved regularity)

Suppose $u \in W(0, T ; V)$ is the weak solution of (2.6), where $\mathcal{L}$ is defined in 2.7. Suppose that the coefficients of $\mathcal{L}$ satisfies Assumptions 2.15 and

$$
a_{t}(t, x) \in L^{\infty}\left(Q_{T}\right)
$$


Assume also

$$
u_{0} \in V, f \in L^{2}\left(0, T ; L^{2}(\Omega)\right)
$$

Then

$$
u \in L^{2}\left(0, T, H^{2}(\Omega)\right) \cap L^{\infty}(0, T ; V), u^{\prime} \in L^{2}\left(0, T ; L^{2}(\Omega)\right),
$$

and we have the estimate

$$
\begin{aligned}
\underset{[0, T]}{\operatorname{ess} \sup _{[0,}\|u(t)\|_{V}} & +\|u\|_{L^{2}\left(0, T ; H^{2}(\Omega)\right)}+\left\|u^{\prime}\right\|_{L^{2}\left(0, T ; L^{2}(\Omega)\right)} \leq \\
& \leq C\left(\|f\|_{L^{2}\left(0, T ; L^{2}(\Omega)\right)}+\left\|u_{0}\right\|_{V}\right) .
\end{aligned}
$$

Proof. (Following the proof of Theorem 5, p. 360, [12].)

Multiplying the equation (2.15) by $d_{m}^{k^{\prime}}(t)$, and summing up all $k=1, \cdots, m$ we get

$$
\left(u_{m}^{\prime}, u_{m}^{\prime}\right)+B\left[u_{m}, u_{m}^{\prime}\right]=\left(f, u_{m}^{\prime}\right) \text {, for a. e. } 0 \leq t \leq T .
$$

We have

$$
\begin{array}{r}
B\left[u_{m}, u_{m}^{\prime}\right]=\int_{0}^{1} a(x, t) u_{m, x} u_{m, x}^{\prime}+\left(b(x, t)+a_{x}(x, t)\right) u_{m, x} u_{m}^{\prime}+c(x, t) u_{m} u_{m}{ }^{\prime} d x= \\
=\frac{d}{d t}\left(\frac{1}{2} A\left[u_{m}, u_{m}\right]\right)-\frac{1}{2} \int_{0}^{1} a_{t} u_{m, x} u_{m, x} d x+\int_{0}^{1}\left(b(x, t)+a_{x}(x, t)\right) u_{m, x} u_{m}{ }^{\prime}+c(x, t) u_{m} u_{m}{ }^{\prime} d x=: \mathbf{A}+\mathbf{B},
\end{array}
$$

where $\mathbf{A}=\frac{d}{d t}\left(\frac{1}{2} A\left[u_{m}, u_{m}\right]\right)$ with the symmetric bilinear form

$$
A[u, v]:=\int_{0}^{1} a(x, t) u_{m, x} u_{m, x} d x, u, v \in V
$$

We have

$$
\begin{array}{r}
|\mathbf{B}| \leq\left(C+\frac{C}{\epsilon}\right)\left\|u_{m}\right\|_{V}^{2}+\epsilon\left\|u_{m}^{\prime}\right\|_{L^{2}(\Omega)}^{2}, \text { and }\left|\left(f, u_{m}{ }^{\prime}\right)\right| \leq \frac{C}{\epsilon}\|f\|_{L^{2}(\Omega)}^{2}+\epsilon\left\|u_{m}{ }^{\prime}\right\|_{L^{2}(\Omega)}^{2} \\
\text { for } \epsilon>0 .
\end{array}
$$

Combining the above inequalities and the equation (2.20), we obtain

$$
\begin{array}{r}
\left\|u_{m}^{\prime}\right\|_{L^{2}(\Omega)}^{2}+\frac{d}{d t}\left(\frac{1}{2} A\left[u_{m}, u_{m}\right]\right) \leq \\
\leq\left(\frac{C}{\epsilon}+C\right)\left\|u_{m}\right\|_{V}^{2}+\frac{C}{\epsilon}\|f\|_{L^{2}(\Omega)}^{2}+2 \epsilon\left\|u_{m}^{\prime}\right\|_{L^{2}(\Omega)}^{2}
\end{array}
$$

Choosing $\epsilon=\frac{1}{4}$ and integrating with respect to t over $[0, T]$, we get 


$$
\begin{array}{r}
\int_{0}^{T}\left\|u_{m}^{\prime}\right\|_{L^{2}(\Omega)}^{2} d t+\sup _{0 \leq t \leq T} A\left[u_{m}(t), u_{m}(t)\right] \leq \\
\leq C\left(A\left[u_{m}(0), u_{m}(0)\right]+\int_{0}^{T}\left\|u_{m}\right\|_{V}^{2}+\|f\|_{L^{2}(\Omega)}^{2} d t\right) \leq \\
\leq C\left(\left\|u_{0}\right\|_{V}^{2}+\|f\|_{L^{2}\left(0, T ; L^{2}(\Omega)\right)}\right),
\end{array}
$$

according to equation (2.13) and (2.14), we can estimate $\left\|u_{m}(0)\right\|_{V} \leq\left\|u_{0}\right\|_{V}$. Furthermore as $A[u, u] \geq \theta \int_{0}^{1}|D u|^{2} d x$ for all $u \in V$, we have that

$$
\sup _{0 \leq t \leq T}\|u(t)\|_{V}^{2} \leq C\left(\left\|u_{0}\right\|_{V}^{2}+\|f\|_{L^{2}\left(0, T ; L^{2}(\Omega)\right)}\right) .
$$

Passing to limits as $m \rightarrow \infty$, we deduce $u \in L^{\infty}(0, T ; V), u^{\prime} \in L^{2}\left(0, T, L^{2}(\Omega)\right)$ :

$$
\|u\|_{L^{\infty}(0, T ; V)}+\left\|u^{\prime}\right\|_{L^{2}\left(0, T, L^{2}(\Omega)\right)} \leq C\left(\left\|u_{0}\right\|_{V}^{2}+\|f\|_{L^{2}\left(0, T ; L^{2}(\Omega)\right)}\right) .
$$

In order to prove that $u \in L^{2}\left(0, T ; H^{2}(\Omega)\right)$ we recall the equation

$$
\left(u^{\prime}, v\right)+B[u, v]=(f, v), \text { for each } v \in V
$$

We rewrite as

$$
B[u, v]=(h, v),
$$

where $h:=f-u^{\prime}$. As $h(t) \in L^{2}(\Omega)$, for a.e. $0 \leq t \leq T$.

We have for a.e. $0 \leq t \leq T$

$$
u_{x x}=\frac{1}{a(t, x)}\left(b(t, x) u_{x}+c(t, x) u-h\right)
$$

then we deduce the estimate

$$
\|u\|_{H^{2}(\Omega)}^{2} \leq C\left(\|h\|_{L^{2}(\Omega)}^{2}+\left\|u_{x}\right\|_{L^{2}(\Omega)}^{2}+\|u\|_{L^{2}(\Omega)}^{2}\right) \leq C\left(\|f\|_{L^{2}(\Omega)}^{2}+\left\|u^{\prime}\right\|_{L^{2}(\Omega)}^{2}+\|u\|_{L^{2}(\Omega)}^{2}\right)
$$

Integrating the above estimate and using the estimates from (2.26), we complete the proof for this theorem. 


\subsection{Convex analysis}

The aim of this subsection is to provide an overview about convex analysis that are needed in the following. Some notations and basic facts will be introduced, for more details we refer to [7]. For minimizing the functionals in the following, we need to introduce the concept of derivatives of functionals and the optimization methods. They can be regarded as an analogous case to directional or total derivatives of real functions: Gateaux derivative or Fréchet derivative.

Let $\mathrm{X}$ and $\mathrm{Y}$ Banach spaces and a given map $f: U \rightarrow Y$, where $U \subset X$ is an open subset.

Definition 2.24 (G-derivative at a point). The map $f$ is called Gâteaux-differentiable (G-differentiable) at $x \in U$ if there exits a map $T: X \rightarrow Y$ such that

$$
f(x+t h)-f(x)-t T(h)=o(t), t \rightarrow 0,
$$

for all $h \in X$, with $\|h\|_{X}=1$. The map $T=: f^{\prime}(x)$ is the Gateaux derivative of $f$ at $x$ and is defined by $\partial G f(x, h)=f^{\prime}(x) h$.

Definition 2.25 (F-derivative at a point). The map $f$ is called Fréchet-differentiable ( $F$-differentiable) at $x \in U$ if there exits a linear map $T \in L(X, Y)$ such that

$$
f(x+h)-f(x)-T h=R(x, h), \quad \lim _{\|h\|_{X} \rightarrow 0} \frac{\|R(x, h)\|_{Y}}{\|h\|_{X}}=0 .
$$

for all $h \in X$ is some neighborhood of zero. If $T$ exists, The map $f^{\prime}(x):=T$ is called Fréchet derivative of $f$ at $x$ and is defined by $d f(x ; h)=f^{\prime}(x) h$.

Definition 2.26 (F-derivative or $G$-derivative on domain $U$ ). If the Fréchet-derivative (or Gâteauxderivative) exits for all $x \in U$, then the mapping

$$
f^{\prime}: U \subset X \rightarrow L(X, Y), x \longmapsto f^{\prime}(x)
$$

is called Fréchet-derivative ( or Gâteaux-derivative) of $f$ on $U$. 



\section{Chapter 3}

\section{Mathematical models for continuous catalyst positioning in $\mathrm{Li} /$ air batteries}

In this Chapter we study the well-posedness of the two models of continuous catalyst positioning in $\mathrm{Li} /$ air baterries (see Subsection 1.2, equations (1.22) and (1.23)). These forward models are wellposed in a certain set of some functional spaces.

\subsection{Formulation and weak solution of Problem $I_{a}$}

We recall problem $I_{a}$ from (1.20a)-(1.20f) with slight reformulation on $\beta$ and $\gamma$ as follows:

Let $c=c(t, x): Q_{T} \rightarrow \mathbb{R}$ be the oxygen concentration inside the pore and $r=r(t, x): Q_{T} \rightarrow \mathbb{R}$ the pore radius. Then $c$ and $r$ satisfy the system of partial differential equations

$$
\begin{gathered}
\frac{\partial}{\partial t}\left(r^{2} c\right)=\frac{\partial}{\partial x}\left(r^{2} \frac{\partial c}{\partial x}\right)-\beta(t, r, c) r c,(t, x) \in Q_{T}, \\
\frac{\partial r}{\partial t}=-\gamma(t, r, c) \operatorname{cat}(x) c,(t, x) \in Q_{T}
\end{gathered}
$$

With initial conditions:

$$
\begin{gathered}
r(0, x)=r_{0}(x)=1, x \in \Omega, \\
c(0, x)=h(x),
\end{gathered}
$$

and mixed boundary conditions:

$$
\begin{gathered}
c(t, 0)=1, t \in[0, T], \\
c_{x}(t, 1)=0, t \in[0, T],
\end{gathered}
$$


the functions $h(x)$ and $\operatorname{cat}(x)$ are supposed to satisfy

$$
\begin{aligned}
& h(0)=1, h(1)=0, \\
& h \in W^{2, \infty}(\Omega), \\
& \text { cat } \in W^{1, \infty}(\Omega),
\end{aligned}
$$

where

$$
\begin{aligned}
& \beta(t, r, c)=\frac{D_{1}}{\int_{0}^{1} r H_{\epsilon}(c) d x}, \\
& \gamma(t, r, c)=\frac{D_{2}}{\int_{0}^{1} r H_{\epsilon}(c) d x},
\end{aligned}
$$

with $\epsilon$ being a small positive number. The constants $D_{1}, D_{2}$ are some given positive numbers. The function $H_{\epsilon}$ has the following form

$H_{\epsilon}(x)=\left\{\begin{array}{l}x, \text { if } x \geq-\frac{a}{\gamma}+c_{0}, \\ 2 \epsilon-\epsilon\left[\frac{2 \gamma-1}{4 \gamma}-\frac{\gamma x}{2}-\frac{\gamma}{2}\left(\gamma x-\gamma c_{0}+a\right)\left(\gamma x-\gamma c_{0}+b\right)+\frac{\gamma^{3}}{2}\left(\gamma x-\gamma c_{0}+a\right)^{2}\left(\gamma x-\gamma c_{0}+b\right)^{2}\right], \\ \text { if }-\frac{b}{\gamma}+c_{0}<x \leq-\frac{a}{\gamma}+c_{0}, \\ \epsilon, \text { otherwise, }\end{array}\right.$

where

$$
a:=1-\frac{1}{2 \gamma}, b:=1+\frac{1}{2 \gamma}, \gamma>0 .
$$

$H_{\epsilon}(x) \geq \epsilon, \forall x \in \mathbb{R}, H_{\epsilon}$ is monotone, $C^{2}$ continuously differentiable (see Appendix A.2 and [10]). The graphic of $H_{\epsilon}(x)$ is plotted in Figure A.4.

Remark 3.1 $H_{\epsilon}(u)$ is bounded in $L^{\infty}\left(Q_{T}\right)$.

Supposing additionally that $r$ and $c$ are smooth enough and $0<r_{\alpha} \leq r, \forall(t, x) \in Q_{T}$, after expanding the derivatives in the both sides of (3.1), and dividing the both sides by $r^{2}$, we get

$$
c_{t}-c_{x x}-\frac{2 r_{x} c_{x}}{r}+\frac{\left(2 r_{t}+\frac{D_{1}}{\int_{0}^{1} r H_{\epsilon}(c) d x}\right) c}{r}=0,(t, x) \in Q_{T} .
$$

We will transform the non-homogeneous problem (3.11), (3.4)-(3.6) in a homogeneous one by setting

$$
u(t, x)=c(t, x)-1
$$


Then $u(t, 0)=u_{x}(t, 1)=0$. Denoting $u_{0}(x)=h(x)-1$, the equation (3.11) leads to

$$
u_{t}-u_{x x}-\frac{2 r_{x} u_{x}}{r}+\left(\frac{2 r_{t}}{r}+\frac{D_{1}}{r \int_{0}^{1} r H_{\epsilon}(u+1) d x}\right) u=-\left(\frac{2 r_{t}}{r}+\frac{D_{1}}{r \int_{0}^{1} r H_{\epsilon}(u+1) d x}\right),(t, x) \in Q_{T} \text {. }
$$

Inserting $r_{t}$ from (3.4) as $r_{t}=-\frac{D_{2}}{\int_{0}^{1} r H_{\epsilon}(u+1) d x} \operatorname{cat}(x)(u+1),(t, x) \in Q_{T}$ into the right hand side of (3.13), then the problem (3.1) - (3.6) leads to the following problem

$$
\begin{gathered}
u_{t}-u_{x x}-\frac{2 r_{x} u_{x}}{r}+\left(\frac{2 r_{t}}{r}+\frac{D_{1}-2 D_{2} \operatorname{cat}(x)}{r \int_{0}^{1} r H_{\epsilon}(u+1) d x}\right) u=\frac{2 D_{2} \operatorname{cat}(x)-D_{1}}{r \int_{0}^{1} r H_{\epsilon}(u+1) d x},(t, x) \in Q_{T}, \\
r_{t}=-\frac{D_{2}}{\int_{0}^{1} r H_{\epsilon}(u+1) d x} \operatorname{cat}(x)(u+1),(t, x) \in Q_{T}, \\
r(0, x)=r_{0}(x)=1, x \in \Omega, \\
u(0, x)=u_{0}(x), x \in \Omega, \\
u(t, 0)=u_{x}(t, 1)=0, t \in[0, T] .
\end{gathered}
$$

We begin a study of this problem by defining a weak solution.

Definition 3.2 A pair $(r, u) \in W^{1, \infty}\left(Q_{T}\right) \times W\left(0, T ; H^{1}(\Omega)\right)$ is said to be a weak solution of (3.14), (3.15),(3.3), (3.16), and (3.17) if the following equalities are satisfied

$$
\begin{array}{r}
\left(u_{t}, v\right)_{\left(\left(H^{1}(\Omega)\right)^{*}, H^{1}(\Omega)\right)}+\int_{0}^{1} u_{x} v_{x} d x-\int_{0}^{1} \frac{2 r_{x} u_{x}}{r} v d x+\int_{0}^{1}\left(\frac{2 r_{t}}{r}+\frac{D_{1}-2 D_{2} \operatorname{cat}(x)}{r \int_{0}^{1} r H_{\epsilon}(u+1) d x}\right) u v d x= \\
=\int_{0}^{1} \frac{2 D_{2} \operatorname{cat}(x)-D_{1}}{r \int_{0}^{1} r H_{\epsilon}(u+1) d x} v d x, \\
\forall v \in \text {, a.e.t } \in[0, T],
\end{array}
$$

where $V$ is defined in Chapter 2.

$$
\begin{gathered}
u(0, x)=u_{0}(x), x \in[0,1] . \\
r(t, x)=1-\int_{0}^{t} \frac{D_{2}}{\int_{0}^{1} r H_{\epsilon}(u+1) d x} \operatorname{cat}(x)(u(\tau, x)+1) d \tau, t \in[0, T] .
\end{gathered}
$$




\subsection{The local existence and uniqueness of the weak solution of problem $I_{a}$}

To prove that there exists a solution to the system (3.14), (3.15),(3.3), (3.16), and (3.17), we will apply the Banach fixed point theorem. For this purpose, we define following spaces

$$
\mathbf{X}_{T}=\left\{r \in L^{\infty}\left(Q_{T}\right) ; r_{x}, r_{t} \in L^{2}\left(0, T, L^{\infty}(\Omega)\right)\right\},
$$

with the norm

$$
\|r\|_{\mathbf{X}_{T}}=\|r\|_{L^{\infty}\left(Q_{T}\right)}+\left\|r_{t}\right\|_{L^{2}\left(0, T, L^{\infty}(\Omega)\right)}+\left\|r_{x}\right\|_{L^{2}\left(0, T, L^{\infty}(\Omega)\right)},
$$

and

$$
\mathbf{Y}_{T}=\left\{u \in L^{2}\left(0, T ; H^{2}(\Omega)\right) \cap L^{\infty}\left(0, T ; H^{1}(\Omega)\right), u^{\prime} \in L^{2}\left(0, T ; L^{2}(\Omega)\right)\right\},
$$

with the norm

$$
\|u\|_{\mathbf{Y}_{T}}=\|u\|_{L^{2}\left(0, T ; H^{2}(\Omega)\right)}+\|u\|_{L^{\infty}\left(0, T ; H^{1}(\Omega)\right)}+\left\|u^{\prime}\right\|_{L^{2}\left(0, T ; L^{2}(\Omega)\right)},
$$

where $u^{\prime}, u^{\prime \prime}$ denoted by $\frac{d u}{d t}, \frac{d^{2} u}{d t^{2}}$ respectively. With $M, r_{\alpha}, \epsilon$ are some given positive constants we define the set

$$
\boldsymbol{M}_{T}=\left\{(r, u), r \in \mathbf{X}_{T}, u \in \mathbf{Y}_{T},\|r-1\|_{\mathbf{X}_{T}}+\left\|u-u_{0}\right\|_{\mathbf{Y}_{T}} \leq M ; r_{\alpha} \leq r \leq R ; \forall \text { a.e. }(t, x) \in Q_{T}\right\},
$$

where $0<r_{\alpha}<1<R$.

Remark 3.3 $\boldsymbol{M}_{T}$ is closed, convex and bounded in $\mathbf{X}_{T} \times \mathbf{Y}_{T}$.

For $(\bar{r}, \bar{u}) \in \boldsymbol{M}_{T}$ define the operator $\mathcal{T}$

$$
\mathcal{T}: \boldsymbol{M}_{T} \longrightarrow \mathbf{X}_{T} \times \mathbf{Y}_{T}
$$

by $(r, u)=\mathcal{T}(\bar{r}, \bar{u})$, where

$$
r(t, x)=1-\int_{0}^{t} \frac{D_{2}}{\int_{0}^{1} \bar{r} H_{\epsilon}(\bar{u}+1) d x} \operatorname{cat}(x)(\bar{u}+1) d \tau .
$$

and $u$ is the solution to the parabolic equation

$$
u_{t}+\mathcal{L} u=f,
$$

with the initial and boundary condition (3.16), (3.17). Here

$$
\begin{array}{r}
\mathcal{L} u=-u_{x x}-\frac{2 r_{x} u_{x}}{r}+\left(\frac{2 r_{t}}{r}+\frac{D_{1}-2 D_{2} \operatorname{cat}(x)}{r \int_{0}^{1} r H_{\epsilon}(\bar{u}+1) d x}\right) u, \\
\text { and } f=\frac{2 D_{2} \operatorname{cat}(x)-D_{1}}{r \int_{0}^{1} r H_{\epsilon}(\bar{u}+1) d x} .
\end{array}
$$


We will employ the Banach fixed point theorem to prove the existence of a fixed point of the system (3.21), (3.22) with the conditions (3.16), (3.17). We have the following result:

Theorem 3.4 Under the conditions in (3.7), which are cat $\in W^{1, \infty}(\Omega)$ and $u_{0}=h-1 \in W^{2, \infty}(\Omega)$, there exist exactly one weak solution $(u, r)$ on $\boldsymbol{M}_{T}$ of (3.14), (3.15),(3.3), (3.16), and (3.17), where $T$ is small enough.

The idea of using fixed point technique we take from the Master Thesis of Grutzner [17]

The scheme of the proof will proceed as below:

1) $\mathcal{T}$ is defined on $\boldsymbol{M}_{T}$ and maps $\boldsymbol{M}_{T}$ into $\mathbf{X}_{T} \times \mathbf{Y}_{T}$

2) There exists $T$ small enough such that $\mathcal{T}$ maps $\boldsymbol{M}_{T}$ to $\boldsymbol{M}_{T}$.

3) There exists $T$ small enough such that $\mathcal{T}$ is $s$-contractive in $\mathbf{X}_{T} \times \mathbf{Y}_{T}$, i.e

$$
\left\|\mathcal{T}\left(u_{1}, r_{1}\right)-\mathcal{T}\left(u_{2}, r_{2}\right)\right\|_{\mathbf{x}_{T} \times \mathbf{Y}_{T}} \leq s\left\|\left(u_{1}, r_{1}\right)-\left(u_{2}, r_{2}\right)\right\|_{\mathbf{x}_{T} \times \mathbf{Y}_{T}},
$$

for all $\left(u_{1}, r_{1}\right),\left(u_{2}, r_{2}\right) \in \boldsymbol{M}_{T}$, and for $0 \leq s<1$.

\subsection{1 $\mathcal{T}$ is well defined in $M_{T}$ and maps $M_{T}$ into $X_{T} \times Y_{T}$ for $\mathbf{T}$ small enough}

Proposition 3.5 With $(\bar{r}, \bar{u}) \in M_{T}$, cat $(x)$ fixed and satisfies (3.7), the followings hold

(i)

$$
\left\|r_{t}\right\|_{L^{\infty}} \leq C\left(M, T, \epsilon, r_{\alpha}, c a t\right) .
$$

(ii)

$$
\left\|r_{x}\right\|_{L^{\infty}(Q)} \leq C\left(M, T, \epsilon, r_{\alpha}, c a t\right) .
$$

$C\left(M, T, \epsilon, r_{\alpha}, c a t\right)$ in (i) and (ii) is a positive constant depending on $M, T, \epsilon, r_{\alpha}$, cat.

(iii) There exists a positive constant $T_{1}$ such that

$$
r>r_{\alpha}, \forall(t, x) \in\left[0, T_{1}\right] \times[0,1] .
$$

(iv) The coefficients of the operator $\mathcal{L}$ in (3.23) are belong to $L^{\infty}\left(Q_{T_{1}}\right)$.

Proof.

(i) From (3.21), with $(\bar{r}, \bar{u}) \in \boldsymbol{M}_{T}$, using triangle inequality, Hölder's inequality and equation (3.21), 
we get

$$
\begin{aligned}
& \left\|r_{x}\right\|_{L^{\infty}\left(Q_{T}\right)}=\left\|\left(1-\int_{0}^{t} \frac{D_{2}}{\int_{0}^{1} \bar{r} H_{\epsilon}(\bar{u}+1) d x} \operatorname{cat}(x)(\bar{u}+1) d \tau\right)\right\|_{x} \|_{L^{\infty}\left(Q_{T}\right)}= \\
& =\sup _{t, x}\left|\int_{0}^{t}\left(\frac{D_{2}}{\int_{0}^{1} \bar{r} H_{\epsilon}(\bar{u}+1) d x} c a t_{x}(\bar{u}+1)+\frac{D_{2}}{\int_{0}^{1} \bar{r} H_{\epsilon}(\bar{u}+1) d x} \operatorname{cat}(x) \bar{u}_{x}\right) d \tau\right| \leq \\
& \leq \frac{D_{2}}{r_{\alpha} \epsilon}\|c a t\|_{L^{\infty}(\Omega)} \sup _{x} \int_{0}^{T}|\bar{u}+1| d t+\frac{D_{2}}{r_{\alpha} \epsilon}\left\|c a t_{x}\right\|_{L^{\infty}(\Omega)} \sup _{x} \int_{0}^{T}\left|\bar{u}_{x}\right| d t \leq \\
& \leq C\left(\epsilon, r_{\alpha}, \text { cat } \sup _{x}\left(\int_{0}^{T}|\bar{u}| d t+\int_{0}^{T}\left|\bar{u}_{x}\right| d t+T\right) \leq\right. \\
& \leq C\left(\epsilon, r_{\alpha}, \text { cat }\right) \sup _{x}\left(\int_{0}^{T}|\bar{u}| d t+\left(T \int_{0}^{T}\left|\bar{u}_{x}\right|^{2} d t\right)^{\frac{1}{2}}+T\right) \leq \\
& \leq C\left(M, T, \epsilon, r_{\alpha}, \text { cat }\right) .
\end{aligned}
$$

In the last step in the above inequality we use result (2.26) in Theorem 2.23.

(ii) Further,

$$
\left\|r_{t}\right\|_{L^{\infty}\left(Q_{T}\right)}=\left\|\frac{D_{2}}{\int_{0}^{1} \bar{r} H_{\epsilon}(\bar{u}+1) d x} \operatorname{cat}(x)(\bar{u}+1)\right\|_{L^{\infty}(Q)} \leq C\left(M, T, \epsilon, r_{\alpha}, c a t\right) .
$$

(iii) Finally, due to (3.21), we see that $r \geq r_{\alpha}$, if

$$
\int_{0}^{t} \frac{D_{2}}{\int_{0}^{1} \bar{r} H_{\epsilon}(\bar{u}+1) d x} \operatorname{cat}(x)(\bar{u}+1) d \tau \leq 1-r_{\alpha} .
$$

Meanwhile, we have the following estimate

$$
\int_{0}^{t} \frac{D_{2}}{\int_{0}^{1} \bar{r} H_{\epsilon}(\bar{u}+1) d x} \operatorname{cat}(x)(\bar{u}+1) d \tau \leq t C\left(M, T, \epsilon, r_{\alpha}, c a t\right) .
$$

Therefore by choosing $T_{1}<\frac{1-r_{\alpha}}{2 C\left(M, T, \epsilon, r_{\alpha}, C a t\right)}$, we arrive at (3.24), which is required to prove.

Remark 3.6 With $(\bar{r}, \bar{u}) \in \boldsymbol{M}_{T_{1}}, f$ in (3.23) belongs to $L^{2}\left(Q_{T_{1}}\right)$.

Remark 3.7 With h satisfying (3.7), the function $u_{0} \in V$.

Theorems 2.21 and 2.22 follow the following results. 
Theorem 3.8 Suppose that $(\bar{r}, \bar{u}) \in \boldsymbol{M}_{T_{1}}$. There exists a unique solution $u \in W\left(0, T_{1}\right)$ of (3.22), (3.16),(3.17).

Theorem 3.9 (Improved regularity) Suppose that $(\bar{r}, \bar{u}) \in \boldsymbol{M}_{T_{1}}$ and $u \in W\left(0, T_{1} ; V\right)$, is the weak solution of (3.22), (3.16),(3.17). Then

$$
u \in L^{2}\left(0, T_{1}, H^{2}(\Omega)\right) \cap L^{\infty}\left(0, T_{1} ; H_{0}^{1}(\Omega)\right), u^{\prime} \in L^{2}\left(0, T_{1} ; L^{2}(\Omega)\right),
$$

and we have estimate

$$
\begin{aligned}
\operatorname{ess} \sup _{\left[0, T_{1}\right]}\|u(t)\|_{H^{1}(\Omega)} & +\|u\|_{L^{2}\left(0, T_{1} ; H^{2}(\Omega)\right)}+\left\|u^{\prime}\right\|_{L^{2}\left(0, T_{1} ; L^{2}(\Omega)\right)} \leq \\
& \leq C\left(M, T_{1}, \epsilon, r_{\alpha}, \text { cat }\right)\left(\|f\|_{L^{2}\left(0, T_{1} ; L^{2}(\Omega)\right)}+\left\|u_{0}\right\|_{V}\right) .
\end{aligned}
$$

For convenience for further proof, we suppose $T_{1} \leq 1$.

The following lemmas are employed from the above theorems.

Lemma 3.10 Assume $(\bar{r}, \bar{u}) \in \boldsymbol{M}_{T_{1}}$, then the weak solution $u$ of the equation (3.22), (3.16), (3.17) satisfies following estimate

$$
\begin{aligned}
& \text { ess } \sup _{\left[0, T_{1}\right]}\left\|u-u_{0}\right\|_{H^{1}(\Omega)}+\left\|u-u_{0}\right\|_{L^{2}\left(0, T_{1} ; H^{2}(\Omega)\right)}+\left\|\left(u-u_{0}\right)^{\prime}\right\|_{L^{2}\left(0, T_{1} ; L^{2}(\Omega)\right)} \leq \\
& \leq C\left(M, T_{1}, \epsilon, r_{\alpha}, u_{0}, \text { cat }\right) \sqrt{T_{1}}
\end{aligned}
$$

Proof.

We see that $\left(u-u_{0}\right)$ is the solution of the equation

$$
w_{t}-\mathcal{L} w=f_{w},
$$

with homogeneous initial and mixed-boundary conditions. The function

$$
\begin{aligned}
& f_{w}=f-\mathcal{L} u_{0}= \\
= & \frac{2 D_{2} \operatorname{cat}(x)-D_{1}}{r \int_{0}^{1} r H_{\epsilon}(\bar{u}+1) d x}+\left(u_{0}\right)_{x x}+\frac{2 r_{x}\left(u_{0}\right)_{x}}{r}-\left(2 r_{t}+\frac{D_{1}}{\int_{0}^{1} r H_{\epsilon}(\bar{u}+1) d x}-\frac{2 D_{2} \operatorname{cat}(x)}{\int_{0}^{1} r H_{\epsilon}(\bar{u}+1) d x}\right) u_{0} .
\end{aligned}
$$

Applying Theorem 3.9 to the equation (3.27), we deduce the two estimates

ess $\sup _{\left[0, T_{1}\right]}\left\|u-u_{0}\right\|_{H^{1}(\Omega)}+\left\|u-u_{0}\right\|_{L^{2}\left(0, T_{1} ; H^{2}(\Omega)\right)}+\left\|\left(u-u_{0}\right)^{\prime}\right\|_{L^{2}\left(0, T_{1} ; L^{2}(\Omega)\right)} \leq C\left(M, T_{1}, \epsilon, r_{\alpha}\right.$, cat $)\left\|f_{w}\right\|_{L^{2}\left(0, T_{1} ; L^{2}(\Omega)\right)}$, 
Using Proposition 3.5, we estimate

$$
\begin{aligned}
& \left\|f_{w}\right\|_{L^{2}\left(0, T_{1} ; L^{2}(\Omega)\right)} \leq\left\|\frac{2 D_{2} \operatorname{cat}(x)-D_{1}}{r \int_{0}^{1} r H_{\epsilon}(\bar{u}+1) d x}\right\|_{L^{2}\left(0, T_{1} ; L^{2}(\Omega)\right)}+ \\
& +\left\|\left(u_{0}\right)_{x x}+\frac{2 r_{x}\left(u_{0}\right)_{x}}{r}-\left(\frac{2 r_{t}}{r}+\frac{D_{1}}{r \int_{0}^{1} r H_{\epsilon}(\bar{u}+1) d x}-\frac{2 D_{2} \operatorname{cat}(x)}{r \int_{0}^{1} r H_{\epsilon}(\bar{u}+1) d x}\right) u_{0}\right\|_{L^{2}\left(0, T_{1} ; L^{2}(\Omega)\right)} \leq \\
& \leq C\left(M, T_{1}, \epsilon, r_{\alpha}, u_{0}, c a t\right) \sqrt{T_{1}} .
\end{aligned}
$$

Thus we arrive at (3.26).

It follows from this lemma and the continuous embedding $H^{1}(\Omega) \subset L^{\infty}(\Omega)$ the following statement.

Corollary 3.11 The weak solution u of problem (3.22), (3.16), (3.17) satisfies following estimates

(i)

$$
\|u\|_{L^{\infty}\left(Q_{T_{1}}\right)}=\operatorname{ess} \sup _{t, x}|u| \leq C\left(M, T_{1}, \epsilon, r_{\alpha}, u_{0}, c a t\right) .
$$

(ii)

$$
\left\|u_{x}\right\|_{L^{2}\left(0, T ; L^{\infty}(\Omega)\right)} \leq C\left(M, T_{1}, \epsilon, r_{\alpha}, u_{0}, c a t\right)
$$

(iii)

$$
\left\|u_{x}\right\|_{L^{\infty}\left(0, T ; L^{2}(\Omega)\right)} \leq C\left(M, T_{1}, \epsilon, r_{\alpha}, u_{0}, c a t\right)
$$

\subsubsection{The mapping $\mathcal{T}$ maps $M_{T}$ to $M_{T}$ for $\mathrm{T}$ small enough.}

Lemma 3.12 With small enough $T$, $\mathcal{T}$ maps $\boldsymbol{M}_{T}$ to $\boldsymbol{M}_{T}$.

Proof. The scheme of the proof is as follows

1. The condition $(\bar{r}, \bar{u})$

$$
\|\bar{r}-1\|_{\mathbf{X}_{T}}+\left\|\bar{u}-u_{0}\right\|_{\mathbf{Y}_{T}} \leq M
$$

follows that $(r, u)$ also satisfies

$$
\|r-1\|_{\mathbf{X}_{T}}+\left\|u-u_{0}\right\|_{\mathbf{Y}_{T}} \leq M
$$

2. The condition $r_{\alpha} \leq \bar{r} \leq R$ implies that $r_{\alpha} \leq r \leq R, \forall(t, x) \in Q_{T}$, where $Q_{T}=[0, T] \times[0,1]$, with $T$ small enough.

Now we prove these statements. 
1. We will estimate each term of the norm

$\left\|\left(r-1, u-u_{0}\right)\right\|_{\mathbf{x}_{T} \times \mathbf{Y}_{T}}=\|r-1\|_{\mathbf{X}_{T}}+\left\|u-u_{0}\right\|_{\mathbf{Y}_{T}}=\|r-1\|_{L^{\infty}\left(Q_{T}\right)}+\left\|r_{t}\right\|_{L^{2}\left(0, T ; L^{\infty}(\Omega)\right)}+\left\|r_{x}\right\|_{L^{2}\left(0, T ; L^{\infty}(\Omega)\right)}+$ + ess $\sup _{[0, T]}\left\|u-u_{0}\right\|_{H^{1}(\Omega)}+\left\|u-u_{0}\right\|_{L^{2}\left(0, T ; H^{2}(\Omega)\right)}+\left\|\left(u-u_{0}\right)^{\prime}\right\|_{L^{2}\left(0, T ; L^{2}(\Omega)\right)}$.

From (3.167) and Proposition 3.5, we have

(i) The condition $(\bar{r}, \bar{u}) \in \boldsymbol{M}_{T}$ implies that $\|\bar{u}\|_{L^{\infty}\left(Q_{T}\right)} \leq C\left(M, u_{0}\right)$.Therefore,

$$
\begin{aligned}
& \|r-1\|_{L^{\infty}\left(Q_{T}\right)}=\operatorname{ess} \sup _{t, x}\left|\int_{0}^{t} \frac{D_{2}}{\int_{0}^{1} \bar{r} H_{\epsilon}(\bar{u}+1) d x} \operatorname{cat}(x)(\bar{u}+1) d \tau\right| \leq \\
& \leq T C\left(M, T, u_{0}, r_{\alpha}, \epsilon, c a t\right) \operatorname{ess} \sup _{x, t}(|\bar{u}|+1) \leq \\
& \leq T C\left(M, T, u_{0}, r_{\alpha}, \epsilon, c a t\right) .
\end{aligned}
$$

(ii) Similarly,

$$
\left\|r_{t}\right\|_{L^{2}\left(0, T ; L^{\infty}(\Omega)\right)} \leq \sup _{x}\left(\int_{0}^{T}\left(\frac{D_{2}}{\int_{0}^{1} \bar{r} H_{\epsilon}(\bar{u}+1) d x} \operatorname{cat}(x)(\bar{u}+1)\right)^{2} d t\right)^{\frac{1}{2}} \leq \sqrt{T} C\left(M, T, u_{0}, r_{\alpha}, \epsilon, c a t\right) .
$$

(iii) The condition $(\bar{r}, \bar{u}) \in \boldsymbol{M}_{T}$ implies that $\left\|\bar{u}_{x}\right\|_{L^{2}\left(0, T ; L^{\infty}(\Omega)\right)} \leq C\left(M, u_{0}\right)$. Hence applying the Hölder inequality, we obtain

$$
\begin{aligned}
& \left\|r_{x}\right\|_{L^{2}\left(0, T ; L^{\infty}(\Omega)\right)}^{2} \leq \int_{0}^{T} \sup _{x}\left(1-\int_{0}^{t} \frac{D_{2}}{\int_{0}^{1} \bar{r} H_{\epsilon}(\bar{u}+1) d x} \operatorname{cat}(x)(\bar{u}+1) d \tau\right)_{x}^{2} d t \leq \\
& \leq \int_{0}^{T} \sup _{x}\left(\int_{0}^{t}\left(\frac{C_{2}}{\int_{0}^{1} \bar{r} H_{\epsilon}(\bar{u}+1) d x} \operatorname{cat}_{x}(\bar{u}+1)+\frac{D_{2}}{\int_{0}^{1} \bar{r} H_{\epsilon}(\bar{u}+1) d x} \operatorname{cat}(x) \bar{u}_{x}\right) d \tau\right)^{2} d t \leq \\
& \leq C\left(r_{\alpha}, \epsilon, c a t\right) \int_{0}^{T} \sup _{x, t} \int_{0}^{T}\left(1+|\bar{u}|^{2}+\left|\bar{u}_{x}\right|^{2}\right) d t d t \leq \\
& \leq T C\left(r_{\alpha}, \epsilon, c a t\right)\left(1+\|\bar{u}\|_{L^{\infty}\left(Q_{T}\right)}^{2}+\left\|\bar{u}_{x}\right\|_{L^{2}\left(0, T ; L^{\infty}(\Omega)\right)}^{2}\right) \leq T C\left(M, T, u_{0}, r_{\alpha}, \epsilon, \text { cat }\right) .
\end{aligned}
$$

Therefore

$$
\left\|r_{x}\right\|_{L^{2}\left(0, T ; L^{\infty}(\Omega)\right)} \leq \sqrt{T} C\left(M, T, u_{0}, r_{\alpha}, \epsilon, c a t\right) .
$$

Combining (3.25), (3.34), (3.37), (3.37) we obtain

$$
\|r-1\|_{X_{T}}+\|u\|_{Y_{T}} \leq \sqrt{T} C\left(M, T, u_{0}, r_{\alpha}, \epsilon, c a t\right) .
$$

Then

$$
\|r-1\|+\|u\| \leq M, \forall t<T_{2}
$$


where $T_{2}=\frac{1}{2} \frac{1}{\sqrt{T} C\left(M, T, u_{0}, r_{\alpha}, \epsilon, c a t\right)}$.

2. We have proven in the Proposition 3.5 that $r \geq r_{\alpha}$ for $T$ small enough. From (3.167) it follows that

$$
\begin{aligned}
& r \leq 1+\left|\int_{0}^{t} \frac{D_{2}}{\int_{0}^{1} \bar{r} H_{\epsilon}(\bar{u}+1) d x} \operatorname{cat}(x)(\bar{u}(\tau, x)+1) d \tau\right| \leq \\
& \leq t C\left(M, T, u_{0}, r_{\alpha}, \epsilon, c a t\right)+1
\end{aligned}
$$

Thus $r \leq R$ for $t \in\left[0, T_{3}\right]$, where $T_{3}=\frac{R-1}{2 C\left(M, T, u_{0}, r_{\alpha}, \epsilon, c a t\right)}$. Hence for $T$ satisfying:

$$
T \leq T^{*}=\min \left\{T_{1}, T_{2}, T_{3}\right\}
$$

the $\mathcal{T}$ maps $\boldsymbol{M}_{T}$ into $\boldsymbol{M}_{T}$.

\subsection{3 $\mathcal{T}$ is contractive in the $\mathbf{X}_{T} \times \mathbf{Y}_{T}$ norm with $\mathbf{T}$ small enough.}

Let $\left(\overline{u_{1}}, \overline{r_{1}}\right),\left(\overline{u_{2}}, \overline{r_{2}}\right) \in \boldsymbol{M}_{T^{*}}$, where $T^{*}$ is defined in (3.40). Suppose that

$$
\begin{aligned}
& \mathcal{T}\left(\bar{u}_{1}, \bar{r}_{1}\right)=\left(u_{1}, r_{1}\right), \\
& \mathcal{T}\left(\bar{u}_{2}, \bar{r}_{2}\right)=\left(u_{2}, r_{2}\right) .
\end{aligned}
$$

Then $\bar{u}_{1}, \bar{r}_{1}, u_{1}, r_{1}$ and $\bar{u}_{2}, \bar{r}_{2}, u_{2}, r_{2}$ satisfy the equations (3.14), (3.15), (3.15), (3.16), (3.17) and (3.3). Denoting $\tilde{u}=u_{1}-u_{2}, \tilde{r}=r_{1}-r_{2}$. Subtracting the two parabolic equations and the two differential equations, we have the following system.

$$
\begin{aligned}
& \tilde{u}_{t}-\tilde{u}_{x x}-\frac{r_{1 x}}{r_{1}} \tilde{u}_{x}+\left(\frac{2 r_{1 t}}{r_{1}}+\frac{D_{1}-2 D_{2} \operatorname{cat}(x)}{r_{1} \int_{0}^{1} r_{1} H_{\epsilon}\left(\bar{u}_{1}+1\right) d x}\right) \tilde{u}=\left(\frac{2 r_{1 x}}{r_{1}}-\frac{2 r_{2 x}}{r_{2}}\right) u_{2 x}-\left(\frac{2 r_{1 t}}{r_{1}}-\frac{2 r_{2 t}}{r_{2}}\right) u_{2}+ \\
& +\left(2 D_{2} \operatorname{cat}(x)-D_{1}\right)\left(\frac{1}{r_{1} \int_{0}^{1} r_{1} H_{\epsilon}\left(\bar{u}_{1}+1\right) d x}-\frac{1}{r_{2} \int_{0}^{1} r_{2} H_{\epsilon}\left(\bar{u}_{2}+1\right) d x}\right)\left(u_{2}+1\right)
\end{aligned}
$$

with the initial and boundary conditions

$$
\begin{gathered}
\tilde{u}(0, x)=0, \\
\tilde{u}(t, 0)=\tilde{u}_{x}(t, 1)=0, t \in\left[0, T^{*}\right],
\end{gathered}
$$


coupled with the equation

$\tilde{r}=-D_{2} \operatorname{cat}(x)\left[\int_{0}^{t} \frac{\bar{u}_{1}-\bar{u}_{2}}{\int_{0}^{1} \bar{r}_{1} H_{\epsilon}\left(\bar{u}_{1}+1\right) d x} d \tau+\int_{0}^{t}\left(\bar{u}_{2}+1\right)\left(\frac{1}{\int_{0}^{1} \bar{r}_{1} H_{\epsilon}\left(\bar{u}_{1}+1\right) d x}-\frac{1}{\int_{0}^{1} \bar{r}_{2} H_{\epsilon}\left(\bar{u}_{2}+1\right) d x} d \tau\right)\right]$.

We have the following result.

Lemma 3.13 Suppose that the functions $h$, cat, $H_{\epsilon}$ satisfy (3.7), (A.12), (3.9). The following estimate for the problem (3.42), (3.43), (3.44) on $Q_{T^{*}}=\left[0, T^{*}\right] \times[0,1]$ holds

$$
\begin{aligned}
& \text { ess } \sup _{\left[0, T^{*}\right]}\|\tilde{u}(t)\|_{H^{1}(\Omega)}+\|\tilde{u}\|_{L^{2}\left(0, T^{*} ; H^{2}(\Omega)\right)}+\left\|\tilde{u}^{\prime}\right\|_{L^{2}\left(0, T^{*} ; L^{2}(\Omega)\right)} \leq \\
& \leq C\left(M, T^{*}, \epsilon, r_{\alpha}, u_{0}, c a t\right) \sqrt{T^{*}}\left(\left\|\bar{r}_{1}-\bar{r}_{2}\right\|_{L^{\infty}\left(Q_{T^{*}}\right)}+\left\|\bar{u}_{1}-\bar{u}_{2}\right\|_{L^{2}\left(0, T^{*} ; H^{2}(\Omega)\right)}+\left\|\bar{u}_{1}-\bar{u}_{2}\right\|_{L^{\infty}\left(0, T^{*} ; H^{1}(\Omega)\right)}\right) .
\end{aligned}
$$

Proof. Applying Theorem 3.9 to the parabolic equation (3.42), and denoting by $f_{12}$ its left hand side, we estimate $f_{12}$ in the $L^{2}\left(Q_{T^{*}}\right)$ norm as follows

$$
\begin{aligned}
& \left\|f_{12}\right\|_{L^{2}\left(Q_{T^{*}}\right)} \leq\left\|\left(\frac{2 r_{1 x}}{r_{1}}-\frac{2 r_{2 x}}{r_{2}}\right) u_{2 x}\right\|_{L^{2}\left(Q_{T^{*}}\right)}+\left\|\left(\frac{2 r_{1 t}}{r_{1}}-\frac{2 r_{2 t}}{r_{2}}\right) u_{2}\right\|_{L^{2}\left(Q_{T^{*}}\right)}+ \\
& +\left\|\left(2 D_{2} \operatorname{cat}(x)-D_{1}\right)\left(\frac{1}{r_{1} \int_{0}^{1} r_{1} H_{\epsilon}\left(\bar{u}_{1}+1\right) d x}-\frac{1}{r_{2} \int_{0}^{1} r_{2} H_{\epsilon}\left(\bar{u}_{2}+1\right) d x}\right)\left(u_{2}+1\right)\right\|_{L^{2}\left(Q_{T^{*}}\right)}
\end{aligned}
$$

Due to $(3.30)$

$$
\begin{aligned}
& \left\|f_{12}\right\|_{L^{2}\left(Q_{T^{*}}\right)} \leq C(M, \text { cat })(\underbrace{\left\|\left(\frac{r_{1 x}}{r_{1}}-\frac{r_{2 x}}{r_{1}}\right) u_{2 x}\right\|_{L^{2}\left(Q_{T^{*}}\right)}}_{A_{1}} \underbrace{\left\|\left(\frac{r_{2 x}}{r_{1}}-\frac{r_{2 x}}{r_{2}}\right) u_{2 x}\right\|_{L^{2}\left(Q_{T^{*}}\right)}}_{A_{2}}+\underbrace{\left\|\left(\frac{r_{1 t}}{r_{1}}-\frac{r_{2 t}}{r_{1}}\right) u_{2}\right\|_{L^{2}\left(Q_{T^{*}}\right)}}_{A_{3}}+ \\
& +\underbrace{\left\|\left(\frac{r_{2 t}}{r_{1}}-\frac{r_{2 t}}{r_{2}}\right) u_{2}\right\|_{L^{2}\left(Q_{T^{*}}\right)}}_{A_{2}}+\underbrace{\left\|\left(\frac{1}{r_{1} \int_{0}^{1} r_{1} H_{\epsilon}\left(\bar{u}_{1}+1\right) d x}-\frac{1}{r_{2} \int_{0}^{1} r_{1} H_{\epsilon}\left(\bar{u}_{1}+1\right) d x}\right)\right\|_{L^{2}\left(Q_{T^{*}}\right)}}_{A_{5}}+ \\
& +\underbrace{\left\|\left(\frac{1}{r_{2} \int_{0}^{1} r_{1} H_{\epsilon}\left(\bar{u}_{1}+1\right) d x}-\frac{1}{r_{2} \int_{0}^{1} r_{2} H_{\epsilon}\left(\bar{u}_{1}+1\right) d x}\right)\right\|_{L^{2}\left(Q_{T^{*}}\right)}}_{A_{6}}+ \\
& +\underbrace{\left\|\left(\frac{1}{r_{2} \int_{0}^{1} r_{2} H_{\epsilon}\left(\bar{u}_{1}+1\right) d x}-\frac{1}{r_{2} \int_{0}^{1} r_{2} H_{\epsilon}\left(\bar{u}_{2}+1\right) d x}\right)\right\|_{L^{2}\left(Q_{T^{*}}\right)}}_{A_{7}}) .
\end{aligned}
$$


1. Since $r_{1} \geq r_{\alpha}, r_{2} \geq r_{\alpha}$ and

$$
\begin{aligned}
& r_{1}(t, x)=1-\int_{0}^{t} \frac{D_{2}}{\int_{0}^{1} \bar{r}_{1} H_{\epsilon}\left(\bar{u}_{1}+1\right) d x} \operatorname{cat}(x)\left(\bar{u}_{1}+1\right) d \tau, \\
& r_{2}(t, x)=1-\int_{0}^{t} \frac{D_{2}}{\int_{0}^{1} \bar{r}_{2} H_{\epsilon}\left(\bar{u}_{2}+1\right) d x} \operatorname{cat}(x)\left(\bar{u}_{2}+1\right) d \tau,
\end{aligned}
$$

we have

$$
\begin{aligned}
& A_{1} \leq \frac{1}{r_{\alpha}}\left\|\left(r_{1 x}-r_{2 x}\right) u_{1 x}\right\|_{L^{2}\left(Q_{T^{*}}\right)} \leq \\
& =\frac{1}{r_{\alpha}} D_{2}\|c a t\|_{W^{1, \infty}(\Omega)}\left(\left\|u_{1 x} \int_{0}^{t}\left(\frac{\bar{u}_{1 x}}{\int_{0}^{1} \bar{r}_{1} H_{\epsilon}\left(\bar{u}_{1}+1\right)}-\frac{\bar{u}_{2 x}}{\int_{0}^{1} \bar{r}_{2} H_{\epsilon}\left(\bar{u}_{2}+1\right)}\right) d \tau\right\|_{L^{2}\left(Q_{T^{*}}\right)}+\right. \\
& \left.+\left\|u_{1 x} \int_{0}^{t}\left(\frac{\bar{u}_{1}+1}{\int_{0}^{1} \bar{r}_{1} H_{\epsilon}\left(\bar{u}_{1}+1\right)}-\frac{\bar{u}_{2}+1}{\int_{0}^{1} \bar{r}_{2} H_{\epsilon}\left(\bar{u}_{2}+1\right)}\right) d \tau\right\|_{L^{2}\left(Q_{T^{*}}\right)}\right) \leq \\
& \leq C\left(r_{\alpha}, \epsilon, \text { cat }\right)(\underbrace{\left\|u_{1 x} \int_{0}^{t}\left(\bar{u}_{1 x}-\bar{u}_{2 x}\right) d \tau\right\|_{L^{2}\left(Q_{T^{*}}\right)}}_{A_{11}}+\underbrace{\left\|u_{1 x} \int_{0}^{t}\left(\bar{u}_{2 x}+1\right) \int_{0}^{1}\left(\bar{r}_{1}-\bar{r}_{2}\right) H_{\epsilon}\left(\bar{u}_{1}+1\right) d x d \tau\right\|_{L^{2}\left(Q_{T^{*}}\right)}}_{A_{12}}+ \\
& +\underbrace{\left\|u_{1 x} \int_{0}^{t}\left(\bar{u}_{2 x}+1\right) \int_{0}^{1} \bar{r}_{2}\left(H_{\epsilon}\left(\bar{u}_{2}+1\right)-H_{\epsilon}\left(\bar{u}_{1}+1\right)\right) d \tau\right\|_{L^{2}\left(Q_{T^{*}}\right)}}_{A_{13}}+\underbrace{\left\|u_{1 x} \int_{0}^{t}\left(\bar{u}_{1}-\bar{u}_{2}\right) d \tau\right\|_{L^{2}\left(Q_{T^{*}}\right)}}_{A_{12}}+ \\
& +\underbrace{\left\|u_{1 x} \int_{0}^{t}\left(\bar{u}_{2}+1\right) \int_{0}^{1}\left(\bar{r}_{1}-\bar{r}_{2}\right) H_{\epsilon}\left(\bar{u}_{1}+1\right) d x d \tau\right\|_{L^{2}\left(Q_{T^{*}}\right)}}_{A_{15}}+ \\
& +\underbrace{\left.\left\|u_{1 x} \int_{0}^{t}\left(\bar{u}_{2}+g\right) \int_{0}^{1} \bar{r}_{2}\left(H_{\epsilon}\left(\bar{u}_{2}+1\right)-H_{\epsilon}\left(\bar{u}_{1}+1\right)\right) d \tau\right\|_{L^{2}\left(Q_{T^{*}}\right)}\right)}_{A_{16}} \text {. }
\end{aligned}
$$

Applying the Hölder inequality, taking into account that $u_{2 x}$ satisfies (3.38), and $H^{2}(\Omega)$ continuous embedded in $L^{\infty}(\Omega)$, we get

$$
\begin{aligned}
& \left(A_{11}\right)^{2}=\int_{0}^{T^{*}} \int_{0}^{1} u_{1 x}\left(\int_{0}^{t}\left(\bar{u}_{1 x}-\bar{u}_{2 x}\right) d \tau\right)^{2} d x d t \leq \int_{0}^{T} \int_{0}^{1}\left(\left|u_{1 x}\right|^{2} T \int_{0}^{T^{*}}\left(\bar{u}_{1 x}-\bar{u}_{2 x}\right)^{2} d t\right) d x d t= \\
& =T \int_{0}^{T^{*}} \operatorname{ess} \sup _{x}\left|u_{1 x}\right|^{2} d t \operatorname{ess} \sup _{x} \int_{0}^{T^{*}}\left(\bar{u}_{1 x}-\bar{u}_{2 x}\right)^{2} d t \int_{0}^{1} \operatorname{ess} \sup _{t \in\left[0, T^{*}\right]}\left|u_{1 x}\right|^{2} d x \leq
\end{aligned}
$$




$$
\begin{aligned}
& \leq^{(3.38)} C\left(M, T^{*}, \epsilon, r_{\alpha}, u_{0}, \text { cat }\right) T\left\|\bar{u}_{1 x}-\bar{u}_{2 x}\right\|_{L^{2}\left(0, T^{*} ; L^{\infty}(\Omega)\right)}^{2} \leq \\
& \leq C\left(M, T^{*}, \epsilon, r_{\alpha}, u_{0}, \text { cat }\right) T^{*}\left\|\bar{u}_{1}-\bar{u}_{2}\right\|_{L^{2}\left(0, T^{*} ; H^{2}(\Omega)\right)}^{2}
\end{aligned}
$$

Similarly, as $H_{\epsilon}\left(\bar{u}_{1}+1\right)$ is bounded in $L^{\infty}\left(Q_{T^{*}}\right)$, we have

$$
\begin{aligned}
& \left(A_{12}\right)^{2} \leq C\left\|\bar{r}_{1}-\bar{r}_{2}^{2}\right\|_{L^{\infty}\left(Q_{T^{*}}\right)} \int_{0}^{T} \int_{0}^{1} u_{1 x}\left(\int_{0}^{t}\left(\bar{u}_{2 x}+1\right) d \tau\right)^{2} d x d t \leq \\
& \leq C\left(M, T^{*}, \epsilon, r_{\alpha}, u_{0}, c a t\right) T^{*}\left\|\bar{r}_{1}-\bar{r}_{2}\right\|_{L^{\infty}\left(Q_{T^{*}}\right.}^{2} .
\end{aligned}
$$

Since $H_{\epsilon}$ is Lipschitz continuous with respect to $u$, we get

$$
\begin{aligned}
& \left(A_{13}\right)^{2} \leq C\left\|\bar{u}_{1}-\bar{u}_{2}^{2}\right\|_{L^{\infty}\left(Q_{T^{*}}\right)} \int_{0}^{T^{*}} \int_{0}^{1} u_{1 x}\left(\int_{0}^{t}\left(\bar{u}_{2 x}+g\right) d \tau\right)^{2} d x d t \leq \\
& \leq C\left(M, T, \epsilon, r_{\alpha}, u_{0}, \text { cat }\right) T\left\|\bar{u}_{1}-\bar{u}_{2}\right\|_{L^{\infty}\left(Q_{T^{*}}\right)}^{2}
\end{aligned}
$$

Similarly, for the three remain terms $A_{12}, A_{15}, A_{16}$ in (3.60), we have:

$$
\begin{gathered}
\left(A_{12}\right)^{2} \leq C\left(M, T, \epsilon, r_{\alpha}, u_{0}, c a t\right) T^{*}\left\|\bar{u}_{1}-\bar{u}_{2}\right\|_{L^{\infty}\left(Q_{T^{*}}\right)}^{2}, \\
\left(A_{15}\right)^{2} \leq C\left(M, T, \epsilon, r_{\alpha}, u_{0}, c a t\right) T\left\|\bar{r}_{1}-\bar{r}_{2}\right\|_{L^{\infty}\left(Q_{T^{*}}\right)}^{2},
\end{gathered}
$$

and

$$
\left(A_{15}\right)^{2} \leq C\left(M, T^{*}, \epsilon, r_{\alpha}, u_{0}, c a t\right) T^{*}\left\|\bar{u}_{1}-\bar{u}_{2}\right\|_{L^{\infty}\left(Q_{T^{*}}\right)}^{2}
$$

From $(3.51)-(3.55)$, we conclude that

$$
A_{1} \leq C\left(M, T^{*}, \epsilon, r_{\alpha}, u_{0}, c a t\right) \sqrt{T^{*}}\left(\left\|\bar{r}_{1}-\bar{r}_{2}\right\|_{L^{\infty}\left(Q_{T^{*}}\right)}+\left\|\bar{u}_{1}-\bar{u}_{2}\right\|_{L^{2}\left(0, T^{*} ; H^{2}(\Omega)\right)}\right) .
$$

2. By the same technique as above, we get:

$$
A_{2} \leq C\left(M, T, \epsilon, r_{\alpha}, u_{0}, c a t\right) \sqrt{T^{*}}\left\|\bar{r}_{1}-\bar{r}_{2}\right\|_{L^{\infty}\left(Q_{T^{*}}\right)} .
$$

3. Now we get estimate $A_{3}$ as follows

$$
\begin{aligned}
A_{3} \leq & \frac{1}{r_{\alpha}}\left\|\left(r_{1 t}-r_{2 t}\right) u_{2}\right\|_{L^{2}\left(Q_{T^{*}}\right)} \leq \\
& =\frac{1}{r_{\alpha}} D_{2}\|c a t\|_{W^{1, \infty}(\Omega)}\left\|u_{2} \int_{0}^{t}\left(\frac{\bar{u}_{1}+1}{\int_{0}^{1} \bar{r}_{1} H_{\epsilon}\left(\bar{u}_{1}+1\right)}-\frac{\bar{u}_{2}+1}{\int_{0}^{1} \bar{r}_{2} H_{\epsilon}\left(\bar{u}_{2}+1\right)}\right) d \tau\right\|_{L^{2}\left(Q_{T^{*}}\right)} \leq
\end{aligned}
$$




$$
\begin{aligned}
& \leq \frac{1}{r_{\alpha}} D_{2}\|c a t\|_{W^{1, \infty}(\Omega)}(\underbrace{\left\|u_{2} \int_{0}^{t}\left(\bar{u}_{1}-\bar{u}_{2}\right) d \tau\right\|_{L^{2}\left(Q_{T^{*}}\right)}}_{A_{31}}+\underbrace{\left\|u_{2} \int_{0}^{t}\left(\bar{u}_{2}+1\right) \int_{0}^{1}\left(\bar{r}_{1}-\bar{r}_{2}\right) H_{\epsilon}\left(\bar{u}_{1}+1\right) d x d \tau\right\|_{L^{2}\left(Q_{T^{*}}\right)}}_{A_{32}}+ \\
& +\underbrace{\left.\left\|u_{2} \int_{0}^{t}\left(\bar{u}_{2}+g\right) \int_{0}^{1} \bar{r}_{2}\left(H_{\epsilon}\left(\bar{u}_{2}+1\right)-H_{\epsilon}\left(\bar{u}_{1}+1\right)\right) d \tau\right\|_{L^{2}\left(Q_{T^{*}}\right)}\right)}_{A_{33}}
\end{aligned}
$$

By the same technique as above, we conclude:

$$
A_{3} \leq C\left(M, T^{*}, \epsilon, r_{\alpha}, u_{0}, c a t\right) \sqrt{T^{*}}\left(\left\|\bar{u}_{1}-\bar{u}_{2}\right\|_{L^{\infty}\left(Q_{T^{*}}\right)}+\left\|\bar{r}_{1}-\bar{r}_{2}\right\|_{L^{\infty}\left(Q_{T^{*}}\right)}\right) .
$$

Similarly, we have:

4.

$$
A_{4} \leq C\left(M, T^{*}, \epsilon, r_{\alpha}, u_{0}, c a t\right) \sqrt{T^{*}}\left(\left\|\bar{u}_{1}-\bar{u}_{2}\right\|_{L^{\infty}\left(Q_{T^{*}}\right)}+\left\|\bar{r}_{1}-\bar{r}_{2}\right\|_{L^{\infty}\left(Q_{T^{*}}\right)}\right)
$$

5.

$$
A_{5} \leq C\left(M, T^{*}, \epsilon, r_{\alpha}, u_{0}, c a t\right) \sqrt{T^{*}}\left(\left\|\bar{u}_{1}-\bar{u}_{2}\right\|_{L^{\infty}\left(Q_{T^{*}}\right)}+\left\|\bar{r}_{1}-\bar{r}_{2}\right\|_{L^{\infty}\left(Q_{T^{*}}\right)}\right),
$$

6.

$$
A_{6} \leq C\left(M, T^{*}, \epsilon, r_{\alpha}, u_{0}, c a t\right) \sqrt{T^{*}}\left(\left\|\bar{u}_{1}-\bar{u}_{2}\right\|_{L^{\infty}\left(Q_{T^{*}}\right)}+\left\|\bar{r}_{1}-\bar{r}_{2}\right\|_{L^{\infty}\left(Q_{T^{*}}\right)}\right),
$$

7.

$$
A_{7} \leq C\left(M, T^{*}, \epsilon, r_{\alpha}, u_{0}, c a t\right) \sqrt{T^{*}}\left\|\bar{u}_{1}-\bar{u}_{2}\right\|_{L^{\infty}\left(Q_{T^{*}}\right)} .
$$

Finally,

$$
\begin{aligned}
& \left\|f_{12}\right\|_{L^{2}\left(Q_{T^{*}}\right)} \leq \\
& \leq C\left(M, T, \epsilon, r_{\alpha}, u_{0}, c a t\right) \sqrt{T}\left(\left\|\bar{r}_{1}-\bar{r}_{2}\right\|_{L^{\infty}\left(Q_{T^{*}}\right)}+\bar{u}_{1}-\bar{u}_{2}\left\|_{L^{2}\left(0, T ; H^{2}(\Omega)\right)}+\right\| \bar{u}_{1}-\bar{u}_{2} \|_{L^{2}\left(0, T ; H^{2}(\Omega)\right)}\right),
\end{aligned}
$$

which implies (3.46).

From (3.45), we also obtain the following result: 
Lemma 3.14 The functions $r_{1}, r_{2}$ defined in (3.41)satisfy the estimate:

$$
\left\|r_{1}-r_{2}\right\|_{\mathbf{X}} \leq T^{*} C(M, \epsilon, c a t)\left(\left\|\bar{r}_{1}-\bar{r}_{2}\right\|_{L^{\infty}\left(Q_{T^{*}}\right)}+\left\|\bar{u}_{1}-\bar{u}_{2}\right\|_{L^{\infty}\left(Q_{T^{*}}\right)}\right) .
$$

Proof. It is easily to get the proof from considering the equation (3.45).

Theorem 3.15 There exists a positive number $T^{* *}$ such that $\mathcal{T}$ is s-contractive in $\mathbf{X}_{T} \times \mathbf{Y}_{T}$ if $T \leq T^{* *}$, where $s$ is a positive constant less than 1.

Proof. From the Lemmas 3.13 and 3.13, we get the estimate

$$
\begin{aligned}
& \left\|r_{1}-r_{2}\right\|_{\mathbf{X}_{T^{*}}}+\left\|u_{1}-u_{2}\right\|_{\mathbf{Y}_{T^{*}}} \leq \\
& \leq C\left(M, T^{*}, \epsilon, r_{\alpha}, u_{0}, c a t\right) \sqrt{T^{*}}\left(\left\|\bar{r}_{1}-\bar{r}_{2}\right\|_{L^{\infty}\left(Q_{T^{*}}\right)}+\left\|\bar{u}_{1}-\bar{u}_{2}\right\|_{L^{2}\left(0, T^{*} ; H^{2}(\Omega)\right)}+\left\|\bar{u}_{1}-\bar{u}_{2}\right\|_{L^{\infty}\left(0, T^{*} ; H^{1}(\Omega)\right)}\right) .
\end{aligned}
$$

Then $\mathcal{T}$ is s-contractive in $\mathbf{X}_{T^{*}} \times \mathbf{Y}_{T^{*}}$ norm with $s \leq \frac{1}{2}$, if $T \leq T^{* *}=\min \left\{\frac{1}{2 C^{2}\left(M, T^{*}, \epsilon, r_{\alpha}, u_{0}, c a t\right)}, T^{*}\right\}$.

Finally, the theorem 3.4 is as a result of applying Banach fixed point Theorem, Lemma 2.7 and Theorem 2.10.

\subsubsection{The continuous dependency on the data of the weak solution in $M_{T}$}

Theorem 3.16 Let $(r, u)$ be the weak solution of the system (3.14), (3.15),(3.3), (3.16), and (3.17) on $Q_{T}$. Then it continuously depends on the the data

$$
\|u\|_{\mathbf{Y}_{T}}+\|r\|_{\mathbf{X}} \leq C\left(M, T, \epsilon, r_{\alpha}, c a t\right) \sqrt{T}\left\|u_{0}\right\|_{H^{2}(\Omega)} .
$$

Proof.

In one hand, according to Lemma 3.10,

$$
\begin{aligned}
& \text { ess } \sup _{[0, T]}\|u\|_{H^{1}(\Omega)}+\|u\|_{L^{2}\left(0, T ; H^{2}(\Omega)\right)}+\left\|u^{\prime}\right\|_{L^{2}\left(0, T ; L^{2}(\Omega)\right)} \leq \\
& \leq C\left(M, T, r_{\alpha}, \text { cat }\right) \sqrt{T}\left\|u_{0}\right\|_{H^{2}(\Omega)} .
\end{aligned}
$$

In another hand, from (3.167) and $(r, u) \in M_{T}$, we can obtain the following estimate

$$
\|r-1\| \mathbf{X}_{T} \leq C_{1}\left(M, T, \epsilon, r_{\alpha}, c a t\right) \sqrt{T}\left(\|u\|_{L^{\infty}(Q)}+\left\|u_{x}\right\|_{L^{2}\left(0, T ; L^{\infty}(\Omega)\right)}\right) .
$$

Combine (3.69) and (3.70) we get (3.68) 


\subsection{The continuity of $(r, c)$ in $W^{1, \infty}\left(Q_{T}\right) \times \mathbf{Y}_{T}$ with respect to $\operatorname{cat}(x)$ in} $W^{1, \infty}(\Omega)$ for problem $I_{a}$ with $T$ small enough.

Let $\operatorname{cat}_{1}(x), \operatorname{cat}_{2}(x) \in W^{1, \infty}(\Omega)$, and assume that there exists a positive constant $C_{\text {cat }}$ such that

$$
\begin{aligned}
& \left\|\operatorname{cat}_{1}(x)\right\|_{W^{1, \infty}(\Omega)} \leq C_{c a t}, \\
& \left\|\operatorname{cat}_{2}(x)\right\|_{W^{1, \infty}(\Omega)} \leq C_{c a t} .
\end{aligned}
$$

Suppose that $\left(u_{1}, r_{1}\right),\left(u_{2}, r_{2}\right) \in \boldsymbol{M}_{T}$, where $T \leq T^{* *}$, satisfy problem (3.14), (3.15),(3.3), (3.16), and (3.17). Then we have:

$$
\begin{gathered}
u_{1 t}-u_{1 x x}-\frac{2 r_{1 x} u_{1 x}}{r_{1}}+\left(\frac{2 r_{1 t}}{r_{1}}+\frac{D_{1}-2 D_{2} c a t_{1}(x)}{r_{1} \int_{0}^{1} r_{1} H_{\epsilon}\left(u_{1}+1\right) d x}\right) u_{1}=\frac{2 D_{2} c a t_{1}(x)-D_{1}}{r_{1} \int_{0}^{1} r_{1} H_{\epsilon}\left(u_{1}+1\right) d x},(t, x) \in Q_{T}, \\
r_{1 t}=-\frac{D_{2}}{\int_{0}^{1} r_{1} H_{\epsilon}\left(u_{1}+1\right) d x} \operatorname{cat}_{1}(x)\left(u_{1}+1\right),(t, x) \in Q_{T}, \\
r_{1}(0, x)=r_{0}(x)=1, x \in \Omega, \\
u_{1}(0, x)=u_{0}(x), x \in \Omega \\
u_{1 x}(t, 1)=0, t \in[0, T]
\end{gathered}
$$

and

$$
\begin{gathered}
u_{2 t}-u_{2 x x}-\frac{2 r_{2 x} u_{2 x}}{r_{2}}+\left(\frac{2 r_{2 t}}{r_{2}}+\frac{D_{1}-2 D_{2} c a t_{2}(x)}{r_{2} \int_{0}^{1} r_{2} H_{\epsilon}\left(u_{2}+1\right) d x}\right) u_{2}=\frac{2 D_{2} c a t_{2}(x)-D_{1}}{r_{2} \int_{0}^{1} r_{2} H_{\epsilon}\left(u_{2}+1\right) d x},(t, x) \in Q_{T}, \\
r_{2 t}=-\frac{D_{2}}{\int_{0}^{1} r_{2} H_{\epsilon}\left(u_{2}+1\right) d x} \operatorname{cat}_{1}(x)\left(u_{2}+1\right),(t, x) \in Q_{T}, \\
r_{2}(0, x)=r_{0}(x)=1, x \in \Omega, \\
u_{2}(0, x)=u_{0}(x), x \in \Omega, \\
u_{2 x}(t, 1)=0, t \in[0, T] .
\end{gathered}
$$

Denote $\mathcal{U}=u_{1}-u_{2}, \mathcal{R}=r_{1}-r_{2}$, we have the following Theorem. 
Theorem 3.17 There exist $T$ small enough and positive constant $C\left(M, T, \epsilon, r_{\alpha}, u_{0}, C_{c a t}\right)$ such that

$$
\|\mathcal{U}\|_{\mathbf{Y}_{T}}+\|\mathcal{R}\|_{W^{1, \infty}\left(Q_{T}\right)} \leq C\left(M, T, \epsilon, r_{\alpha}, u_{0}, C_{c a t}\right) \| c a t_{1}-\text { cat }_{2} \|_{W^{1, \infty}(\Omega)},
$$

where

$$
\begin{array}{r}
\|\mathcal{R}\|_{W^{1, \infty}\left(Q_{T}\right)}=\|\mathcal{R}\|_{L^{\infty}\left(Q_{T}\right)}+\left\|\mathcal{R}_{t}\right\|_{L^{\infty}\left(Q_{T}\right)}+\left\|\mathcal{R}_{x}\right\|_{L^{\infty}\left(Q_{T}\right)}, \\
\left\|c a t_{1}-\operatorname{cat}_{2}\right\|_{W^{1, \infty}(\Omega)}=\left\|c a t_{1}-\operatorname{cat}_{2}\right\|_{L^{\infty}(\Omega)}+\left\|c a t_{1 x}-\operatorname{cat}_{2 x}\right\|_{L^{\infty}(\Omega)} .
\end{array}
$$

Proof. Subtracting (3.76) by (3.72), denoting $\mathcal{U}=u_{1}-u_{2}, \mathcal{R}=r_{1}-r_{2}$ we get:

$$
\begin{aligned}
& \mathcal{U}_{t}-\mathcal{U}_{x x}-\frac{2 r_{1 x}}{r_{1}} \mathcal{U}_{x}+\left(\frac{r_{1 t}}{r_{1}}+\frac{D_{1}-2 D_{2} c a t_{2}}{r_{1} \int_{0}^{1} r_{2} H_{\epsilon}\left(u_{2}+1\right) d x}\right) \mathcal{U}= \\
& =\frac{\mathcal{R}_{t} u_{2}}{r_{1}}+\frac{2 u_{2 x} \mathcal{R}_{x}}{r_{1}}-\left(\frac{r_{2 t} u_{2}+2 u_{2 x} r_{2 x}}{r_{1} r_{2}}+\frac{2 D_{2} c a t_{2} u_{2}+2 D_{2} c a t_{2}-D_{1} u_{2}-D_{1}}{r_{1} r_{2} \int_{0}^{1} r_{2} H_{\epsilon}\left(u_{2}+1\right) d x}\right) \mathcal{R}+ \\
& +\frac{D_{1} u_{2}+D_{1}+2 D_{2} c a t_{2} u_{2}+2 D_{2} c a t_{2}}{r_{2} \int_{0}^{1} r_{1} H_{\epsilon}\left(u_{1}+1\right) d x \int_{0}^{1} r_{2} H_{\epsilon}\left(u_{1}+1\right) d x} \int_{0}^{1} \mathcal{R} H_{\epsilon}\left(u_{1}+1\right) d x+\frac{\left(c a t_{1}-c a t_{2}\right)\left(2 D_{2} u_{1}+2 D_{2}\right)}{r_{1} \int_{0}^{1} r_{1} H_{\epsilon}\left(u_{1}+1\right) d x}+ \\
& +\frac{D_{1} u_{2}+D_{1}-2 D_{2} c a t_{2} u_{2}-2 D_{2}}{r_{2} \int_{0}^{1} r_{2} H_{\epsilon}\left(u_{1}+1\right) d x \int_{0}^{1} r_{2} H_{\epsilon}\left(u_{2}+1\right) d x} \int_{0}^{1} r_{2}\left(H_{\epsilon}\left(u_{1}+1\right) d x-H_{\epsilon}\left(u_{2}+1\right) d x\right) .
\end{aligned}
$$

Subtracting (3.77) by (3.73), we have:

$$
\begin{aligned}
& \mathcal{R}_{t}=-D_{2}\left(\frac{\left(c a t_{1}-c a t_{2}\right)\left(u_{1}+1\right)}{\int_{0}^{1} r_{1} H_{\epsilon}\left(u_{1}+1\right) d x}+\frac{c a t_{2} U}{\int_{0}^{1} r_{1} H_{\epsilon}\left(u_{1}+1\right) d x}-\right. \\
& \left.\frac{c a t_{2}\left(u_{2}+1\right) \int_{0}^{1} \mathcal{R} H_{\epsilon}\left(u_{1}+1\right) d x}{\int_{0}^{1} r_{1} H_{\epsilon}\left(u_{1}+1\right) d x \int_{0}^{1} r_{2} H_{\epsilon}\left(u_{1}+1\right) d x}-\frac{c a t_{2}\left(u_{2}+1\right) \int_{0}^{1} r_{2}\left(H_{\epsilon}\left(u_{1}+1\right)-H_{\epsilon}\left(u_{2}+1\right)\right) d x}{\int_{0}^{1} r_{2} H_{\epsilon}\left(u_{1}+1\right) d x \int_{0}^{1} r_{2} H_{\epsilon}\left(u_{2}+1\right) d x}\right) .
\end{aligned}
$$

In one hand, applying Theorem 2.23 to the equation (3.81) with homogeneous initial and mixed boundary conditions, we have

$$
\begin{aligned}
\|\mathcal{U}\|_{Y_{T}} \leq C\left(M, T^{* *}, \epsilon, r_{\alpha}, u_{0}, C_{c a t}\right)\left(\left\|\mathcal{R}_{t}\right\|_{L^{2}\left(Q_{T}\right)}+\right. & \left\|u_{2 x} \mathcal{R}_{x}\right\|_{L^{2}\left(Q_{T}\right)}+\left\|r_{2 t} \mathcal{R}\right\|_{L^{2}\left(Q_{T}\right)}+\left\|u_{2 x} \mathcal{R}\right\|_{L^{2}\left(Q_{T}\right)}+ \\
& \left.+\|\mathcal{R}\|_{L^{2}\left(Q_{T}\right)}+\left\|c a t_{1}-\operatorname{cat}_{2}\right\|_{L^{2}(\Omega)}+\|\mathcal{U}\|_{L^{2}\left(Q_{T}\right)}\right) .
\end{aligned}
$$

From Proposition 3.5, we know that $\mathcal{R} \in W^{1, \infty}\left(Q_{T}\right)$, we estimate:

$$
\left\|u_{2 x} \mathcal{R}_{x}\right\|_{L^{2}\left(Q_{T}\right)} \leq\left\|R_{x}\right\|_{L^{2}\left(0, T ; L^{\infty}(\Omega)\right)}\left\|u_{2 x}\right\|_{L^{\infty}\left(0, T ; L^{2}(\Omega)\right)} \leq M\left\|R_{x}\right\|_{L^{2}\left(0, T ; L^{\infty}(\Omega)\right)} \leq M \sqrt{T}\left\|R_{x}\right\|_{L^{\infty}\left(Q_{T}\right)} .
$$


3.3. The continuity of $(r, c)$ in $W^{1, \infty}\left(Q_{T}\right) \times \mathbf{Y}_{T}$ with respect to $\operatorname{cat}(x)$ in $W^{1, \infty}(\Omega)$ for problem $I_{a}$ with $T$ small enough.

Similarly,

$$
\left\|r_{2 t} \mathcal{R}\right\|_{L^{2}\left(Q_{T}\right)} \leq M \sqrt{T}\|R\|_{L^{\infty}\left(Q_{T}\right)}
$$

and

$$
\left\|u_{2 x} \mathcal{R}\right\|_{L^{2}\left(Q_{T}\right)} \leq M \sqrt{T}\|R\|_{L^{\infty}\left(Q_{T}\right)} .
$$

Putting the estimates (3.84) - (3.86) into (3.83), we obtain:

$\|\mathcal{U}\|_{Y_{T}} \leq C\left(M, T^{* *}, \epsilon, r_{\alpha}, u_{0}, C_{c a t}\right) \sqrt{T}\left(\left\|\mathcal{R}_{t}\right\|_{L^{\infty}\left(Q_{T}\right)}+\left\|\mathcal{R}_{x}\right\|_{L^{\infty}\left(Q_{T}\right)}+\|\mathcal{R}\|_{L^{\infty}\left(Q_{T}\right)}+\left\|c a t_{1}-\operatorname{cat}_{2}\right\|_{L^{\infty}(\Omega)}+\|\mathcal{U}\|_{L^{\infty}\left(Q_{T}\right)}\right)$.

Thus, for $T<T_{4}=\frac{1}{2 C\left(M, T^{* *}, \epsilon, r_{\alpha}, u_{0}, C_{c a t}\right)}$, we deduce:

$$
\|\mathcal{U}\|_{Y_{T}} \leq C\left(M, T^{* *}, \epsilon, r_{\alpha}, u_{0}, C_{c a t}\right) \sqrt{T}\left(\left\|\mathcal{R}_{t}\right\|_{L^{\infty}\left(Q_{T}\right)}+\left\|\mathcal{R}_{x}\right\|_{L^{\infty}\left(Q_{T}\right)}+\|\mathcal{R}\|_{L^{\infty}\left(Q_{T}\right)}+\left\|c a t_{1}-\operatorname{cat}_{2}\right\|_{L^{\infty}(\Omega)}\right) .
$$

In another hand, from the equation (3.82) and the homogeneous initial condition, we have the followings estimates:

$$
\begin{gathered}
\|\mathcal{R}\|_{L^{\infty}\left(Q_{T}\right)} \leq C\left(M, T^{* *}, \epsilon, r_{\alpha}, u_{0}, C_{c a t}\right) T\left(\|\mathcal{U}\|_{L^{\infty}\left(Q_{T}\right)}+\|\mathcal{R}\|_{L^{\infty}\left(Q_{T}\right)}+\left\|c a t_{1}-c a t_{2}\right\|_{L^{\infty}(\Omega)}\right) . \\
\left\|\mathcal{R}_{t}\right\|_{L^{\infty}\left(Q_{T}\right)} \leq C\left(M, T^{* *}, \epsilon, r_{\alpha}, u_{0}, C_{c a t}\right)\left(\|\mathcal{U}\|_{L^{\infty}\left(Q_{T}\right)}+\|\mathcal{R}\|_{L^{\infty}\left(Q_{T}\right)}+\left\|c a t_{1}-c a t_{2}\right\|_{L^{\infty}(\Omega)}\right) . \\
\left\|\mathcal{R}_{x}\right\|_{L^{\infty}\left(Q_{T}\right)} \leq C\left(M, T^{* *}, \epsilon, r_{\alpha}, u_{0}, C_{c a t}\right) T\left(\|\mathcal{U}\|_{L^{\infty}\left(Q_{T}\right)}+\|\mathcal{R}\|_{L^{\infty}\left(Q_{T}\right)}+\left\|c a t_{1 x}-c a t_{2 x}\right\|_{L^{\infty}(\Omega)}\right)+ \\
\quad+C\left(R, r_{\alpha}, \epsilon, M\right)\left(\left\|\mathcal{U}_{x}\right\|_{L^{2}\left(0, T ; L^{\infty}(\Omega)\right)}+\|\mathcal{R}\|_{L^{\infty}\left(Q_{T}\right)}+\mathcal{U}\left\|_{L^{\infty}\left(Q_{T}\right)}+\right\| c a t_{1}-\operatorname{cat}_{2} \|_{L^{\infty}\left(Q_{T}\right)}\right) .
\end{gathered}
$$

Hence,

$$
\begin{aligned}
& \|\mathcal{R}\|_{L^{\infty}\left(Q_{T}\right)}+\left\|\mathcal{R}_{t}\right\|_{L^{\infty}\left(Q_{T}\right)}+\left\|\mathcal{R}_{x}\right\|_{L^{\infty}\left(Q_{T}\right)} \leq \\
& \leq C\left(M, T^{* *}, \epsilon, r_{\alpha}, u_{0}, C_{c a t}\right) T\left(\|\mathcal{U}\|_{L^{\infty}\left(Q_{T}\right)}+\|\mathcal{R}\|_{L^{\infty}\left(Q_{T}\right)}+\left\|c a t_{1 x}-\operatorname{cat}_{2 x}\right\|_{L^{\infty}(\Omega)}+\left\|c a t_{1}-\operatorname{cat}_{2}\right\|_{L^{\infty}\left(Q_{T}\right)}\right)+ \\
& +C\left(M, T^{* *}, \epsilon, r_{\alpha}, u_{0}, C_{c a t}\right)\left(\left\|\mathcal{U}_{x}\right\|_{L^{2}\left(0, T ; L^{\infty}(\Omega)\right)}+\|\mathcal{R}\|_{L^{\infty}\left(Q_{T}\right)}+\mathcal{U}\left\|_{L^{\infty}\left(Q_{T}\right)}+\right\| c a t_{1}-c_{a t}\left\|_{L^{\infty}(\Omega)}+\right\| c a t_{1 x}-c a t_{2 x} \|_{L^{\infty}(\Omega)}\right) \text {. }
\end{aligned}
$$

Moreover, from (3.89), for $T<T_{5}=\frac{1}{2 C\left(M, T^{* *}, \epsilon, r_{\alpha}, u_{0}, C_{c a t}\right)}$, we have the following estimate:

$$
\|\mathcal{R}\|_{L^{\infty}\left(Q_{T}\right)} \leq C\left(M, T^{* *}, \epsilon, r_{\alpha}, u_{0}, C_{c a t}\right) T\left(\|\mathcal{U}\|_{L^{\infty}\left(Q_{T}\right)}+\left\|c a t_{1}-c a t_{2}\right\|_{L^{\infty}(\Omega)}\right) .
$$


Without loss of generality, we assume that $T<1$. Continuing the estimate (3.92) with using (3.93), we obtain:

$$
\begin{aligned}
& \|\mathcal{R}\|_{L^{\infty}\left(Q_{T}\right)}+\left\|\mathcal{R}_{t}\right\|_{L^{\infty}\left(Q_{T}\right)}+\left\|\mathcal{R}_{x}\right\|_{L^{\infty}\left(Q_{T}\right)} \leq \\
& \leq C\left(M, T^{* *}, \epsilon, r_{\alpha}, u_{0}, C_{c} a t\right) T\left(\|\mathcal{U}\|_{L^{\infty}\left(Q_{T}\right)}+\left\|c a t_{1 x}-c_{c a t}\right\|_{2 x}\left\|_{L^{\infty}(\Omega)}+\right\| c a t_{1}-c_{c a t} \|_{L^{\infty}(\Omega)}\right)+ \\
& +C\left(M, T^{* *}, \epsilon, r_{\alpha}, u_{0}, C_{c a t}\right)\left(\left\|U_{x}\right\|_{L^{2}\left(0, T ; L^{\infty}(\Omega)\right)}+\mathcal{U}\left\|_{L^{\infty}\left(Q_{T}\right)}+\right\| c a t_{1}-\operatorname{cat}_{2}\left\|_{L^{\infty}(\Omega)}+\right\| c a t_{1 x}-\operatorname{cat}_{2 x} \|_{L^{\infty}(\Omega)}\right) \text {. }
\end{aligned}
$$

From (3.87), we also see that,

$$
\mathcal{U}\left\|_{L^{\infty}\left(Q_{T}\right)}+\right\| \mathcal{U}_{x}\left\|_{L^{2}\left(0, T ; L^{\infty}(\Omega)\right)} \leq\right\| \mathcal{U} \|_{Y_{T}} \leq
$$

$\leq C\left(R, r_{\alpha}, \epsilon, M\right) C\left(M, T^{* *}, \epsilon, r_{\alpha}, u_{0}, C_{c a t}\right) \sqrt{T}\left(\left\|\mathcal{R}_{t}\right\|_{L^{\infty}\left(Q_{T}\right)}+\left\|\mathcal{R}_{x}\right\|_{L^{\infty}\left(Q_{T}\right)}+\|\mathcal{R}\|_{L^{\infty}\left(Q_{T}\right)}+\left\|c a t_{1}-c_{c a t_{2}}\right\|_{L^{\infty}(\Omega)}\right)$.

As $T<1$, hence $T<\sqrt{T}$, continuing the estimate (3.94) with using (3.95), we obtain:

$$
\begin{aligned}
& \|\mathcal{R}\|_{W^{1, \infty}\left(Q_{T}\right)} \leq C\left(M, T^{* *}, \epsilon, r_{\alpha}, u_{0}, C_{c a t}\right) \sqrt{T}\left(\|\mathcal{R}\|_{W^{1, \infty}\left(Q_{T}\right)}+\left\|c a t_{1}-c_{2 a t_{2}}\right\|_{W^{\infty}(\Omega)}\right)+ \\
& \quad+C\left(R, r_{\alpha}, \epsilon, M\right)\left\|c a t_{1}-c a t_{2}\right\|_{W^{1, \infty}(\Omega)} .
\end{aligned}
$$

From (3.96), for $T<T_{6}=\left(\frac{1}{2 C\left(M, T^{* *}, \epsilon, r_{\alpha}, u_{0}, C_{c a t}\right)}\right)^{2}$, we have:

$$
\left.\|\mathcal{R}\|_{W^{1, \infty}\left(Q_{T}\right)} \leq C\left(M, T^{* *}, \epsilon, r_{\alpha}, u_{0}, C_{c a t}\right)\right)\left\|c a t_{1}-\operatorname{cat}_{2}\right\|_{W^{1, \infty}(\Omega)} .
$$

Putting (3.97) into (3.88), we get:

$$
\left.\|\mathcal{U}\|_{\mathbf{Y}_{T}} \leq C\left(M, T^{* *}, \epsilon, r_{\alpha}, u_{0}, C_{c a t}\right)\right)\left\|c a t_{1}-\operatorname{cat}_{2}\right\|_{W^{1, \infty}(\Omega)} .
$$

Finally, from (3.97), (3.98), with $T<T^{* * *}=\min \left\{T_{4}, T_{5}, T_{6}, T^{* *}\right\}$ we get (3.80).

- Due to the continuous embedding of $W^{1, \infty}\left(Q_{T}\right)$ in $\mathbf{X}_{T}$, from Theorem 3.17, we have the following remark:

Corollary $3.18(r, c)$ is continuous in $\mathbf{X}_{T} \times \mathbf{Y}_{T}$ with respect to cat $(x)$ in $W^{1, \infty}(\Omega)$ for problem $I_{a}$ with $T$ small enough.

\subsection{On the Frechet differentiability of $r$ and $c$ with respect to $\operatorname{cat}(x)$ for problem $I_{a}$}

Definition 3.19 Under the assumptions of Theorem 3.4 we define the state mapping $\mathcal{P}$ as following

$$
\begin{aligned}
\mathcal{P}: W^{1, \infty}(\Omega) & \longrightarrow \mathbf{X}_{T} \times \mathbf{Y}_{T} \\
c a t & \longmapsto \mathcal{P}(c a t)=(r(c a t), c(c a t))
\end{aligned}
$$

From the above section 3.1 and 3.2, we know that $\mathcal{P}$ is defined. In this section we will consider the differentiability of the state mapping. 


\subsubsection{The sensitivity system}

We want to present a sensitivity system of equations to calculate $r_{c a t} \delta$, for this we refer [11].

Let $\mu$ a positive number, we have

$$
\begin{gathered}
r_{t}(c a t)=-\frac{D_{2} c a t c}{\int_{0}^{1} r H_{\epsilon}(c) d x}, \\
r_{t}(c a t+\mu \delta)=-\frac{D_{2}(c a t+\mu \delta) c(c a t+\mu \delta)}{\int_{0}^{1} r(c a t+\mu \delta) H_{\epsilon}(c(c a t+\mu \delta)) d x},
\end{gathered}
$$

where $c, r$ satisfy (3.11), (3.15),(3.3), (3.16), (3.17) and $c(c a t+\delta), r(c a t+\delta)$ satisfy (3.11), (3.15),(3.3), (3.16), (3.17) with replacing cat by $c a t+\mu \delta$.

Substracting (3.101) to (3.100),we get

$r_{t}(c a t+\delta)-r_{t}=\frac{-D_{2}(c a t+\mu \delta) c(c a t+\mu \delta) \int_{0}^{1} r H_{\epsilon}(c) d x+D_{2} D_{2} c a t c \int_{0}^{1} r(c a t+\mu \delta) H_{\epsilon}(c(c a t+\mu \delta)) d x}{\int_{0}^{1} r(c a t+\mu \delta) H_{\epsilon}(c(c a t+\mu \delta)) d x \int_{0}^{1} r H_{\epsilon}(c) d x}$.

Dividing two sides of (3.102) by $\mu$, we obtain

$$
\begin{aligned}
& \left(\frac{r(c a t+\delta)-r}{\mu}\right)_{t}=\frac{-D_{2}\left(\frac{c a t+\mu \delta-c a t}{\mu}\right) c(c a t+\mu \delta)+D_{2} c a t \frac{c(c a t+\mu \delta)-c}{\mu}}{\int_{0}^{1} r(c a t+\mu \delta) H_{\epsilon}(c(c a t+\mu \delta)) d x \int_{0}^{1} r H_{\epsilon}(c) d x}+ \\
& +D_{2} c a t c \int_{0}^{1}\left(\frac{r-r(c a t+\mu \delta)}{\epsilon} H_{\epsilon}(c) d x+\int_{0}^{1} r(c a t+\mu \delta) \frac{H_{\epsilon}(c)-H_{\epsilon}(c(c a t+\mu \delta))}{\mu} d x\right) .
\end{aligned}
$$

Denote

$$
\eta(t, x):=\frac{\partial r}{\partial c a t}(c a t) \delta, \quad \frac{\partial r}{\varphi}(t, x):=\frac{\partial c}{\partial c a t}(c a t) \delta,
$$

letting $\mu \rightarrow 0$ in (3.103), we deduce

$$
\eta_{t}=-\frac{D_{2} c a t \varphi}{\int_{0}^{1} r H_{\epsilon}(c) d x}+\frac{D_{2} c a t c}{\left(\int_{0}^{1} r H_{\epsilon}(c)\right)^{2}} \int_{0}^{1} r H_{\epsilon}^{\prime}(c) \varphi+H_{\epsilon}(c) \eta d x-\frac{D_{2} \delta c}{\int_{0}^{1} r H_{\epsilon}(c) d x},
$$

$\eta$ satisfies the homogeneous initial condition .

Now we consider the parabolic equation (3.11), for $c=c(c a t)$ and $c(c a t+\mu \delta)$, subtracting the two equations

$$
c_{t}-c_{x x}-\frac{2 r_{x} c_{x}}{r}+\frac{\left(2 r_{t}+\frac{D_{1}}{\int_{0}^{1} r H_{\epsilon}(c) d x}\right) c}{r}=0,(t, x) \in Q_{T}
$$




$$
\begin{gathered}
c_{t}(c a t+\mu \delta)-c_{x x}(c a t+\mu \delta)-\frac{2 r_{x}(c a t+\mu \delta) c_{x}(c a t+\mu \delta)}{r(c a t+\mu \delta)}+ \\
\frac{\left(2 r_{t}(c a t+\mu \delta)+\frac{D_{1}}{\int_{0}^{1} r(c a t+\mu \delta) H_{\epsilon}(c(c a t+\mu \delta)) d x}\right) c(c a t+\mu \delta)}{r(c a t+\mu \delta)}=0, \quad(t, x) \in Q_{T},
\end{gathered}
$$

we get

$$
\begin{aligned}
& c_{t}(c a t+\mu \delta) c_{t}-\left(c_{x x}(c a t+\mu \delta)-c_{x x}\right)-\left(\frac{2 r_{x}(c a t+\mu \delta) c_{x}(c a t+\mu \delta)}{r(c a t+\mu \delta)}-\frac{2 r_{x} c_{x}}{r}\right)+ \\
& +\left(\frac{\left(2 r_{t}(c a t+\mu \delta) c(c a t+\mu \delta)\right.}{r(c a t+\mu \delta)}-\frac{\left(2 r_{t} c\right.}{r}\right)+\left(\frac{D_{1} c(c a t+\mu \delta)}{r(c a t+\mu \delta) \int_{0}^{1} r(c a t+\mu \delta) H_{\epsilon}(c(c a t+\mu \delta)) d x}-\frac{D_{1} c}{r \int_{0}^{1} r H_{\epsilon}(c) d x}\right)=0
\end{aligned}
$$

Dividing two sides of (3.108) by $\mu$, we obtain

letting $\mu \rightarrow 0$ in (3.108), we obtain

$$
\begin{aligned}
& \varphi_{t}-\varphi_{x x}-\frac{2 r_{x} \varphi_{x}}{r}+\left(\frac{2 r_{t}}{r}+\frac{D_{1}}{r \int_{0}^{1} r H_{\epsilon}(c) d x}\right) \varphi-\frac{D_{1} c}{r\left(\int_{0}^{1} r H_{\epsilon}(c) d x\right)^{2}} \int_{0}^{1} H_{\epsilon}(c) \eta+r H_{\epsilon}^{\prime}(c) \varphi d x- \\
& -\frac{2 c_{x} \eta_{x}}{r}+\frac{2 c \eta_{t}}{r}+\left(\frac{2 r_{x} c_{x}}{r^{2}}-\frac{2 r_{t} c}{r^{2}}-\frac{D_{1} c}{r^{2} \int_{0}^{1} r H_{\epsilon}(c) d x}\right) \eta=0
\end{aligned}
$$

Putting (3.105) into (3.109), we deduce

$$
\begin{aligned}
& \varphi_{t}-\varphi_{x x}-\frac{2 r_{x} \varphi_{x}}{r}+\left(\frac{2 r_{t}}{r}+\frac{D_{1}}{r \int_{0}^{1} r H_{\epsilon}(c) d x}-\frac{2 c D_{2} c a t}{\int_{0}^{1} r H_{\epsilon}(c) d x}\right) \varphi= \\
& =\left(\frac{2 D_{2} c a t c^{2}}{r\left(\int_{0}^{1} r H_{\epsilon}(c)\right)^{2}}-\frac{D_{1} c}{r\left(\int_{0}^{1} r H_{\epsilon}(c) d x\right)^{2}}\right) \int_{0}^{1} H_{\epsilon}(c) \eta+r H_{\epsilon}^{\prime}(c) \varphi d x+ \\
& +\frac{2 c_{x} \eta_{x}}{r}-\left(\frac{2 r_{x} c_{x}}{r^{2}}-\frac{2 r_{t} c}{r^{2}}-\frac{D_{1} c}{r^{2} \int_{0}^{1} r H_{\epsilon}(c) d x}\right) \eta+\frac{2 D_{2} \delta c^{2}}{r \int_{0}^{1} r H_{\epsilon}(c) d x},
\end{aligned}
$$

$\varphi$ satisfies the homogeneous initial and mixed boundary conditions.

Definition 3.20 (The sensitivity system)

The system (3.105), (3.110) with the homogeneous initial and mixed boundary conditions:

$$
\begin{aligned}
& \eta(0, x)=0, x \in[0,1], \\
& \varphi(0, x)=0, x \in[0,1],
\end{aligned}
$$




$$
\begin{aligned}
\varphi(t, 0) & =0, t \in[0, T], \\
\varphi_{x}(t, 1) & =0, t \in[0, T],
\end{aligned}
$$

is called sensitivity system.

We define a weak solution.

Definition 3.21 A pair $(\eta, \varphi) \in W^{1, \infty}\left(Q_{T}\right) \times W\left(0, T ; H^{1}(\Omega)\right)$ is said to be a weak solution of the sensitivity system that is defined in Definition 3.20 if the following equalities are satisfied

$$
\begin{gathered}
\left(\varphi_{t}, v\right)_{\left(\left(H^{1}(\Omega)\right)^{*}, H^{1}(\Omega)\right)}+\int_{0}^{1} \varphi_{x} v_{x} d x-\int_{0}^{1} \frac{2 r_{x} \varphi_{x}}{r} v d x+\int_{0}^{1}\left(\frac{2 r_{t}}{r}+\frac{D_{1}}{r \int_{0}^{1} r H_{\epsilon}(c) d x}-\frac{2 c D_{2} c a t}{\int_{0}^{1} r H_{\epsilon}(c) d x}\right) \varphi v d x= \\
\quad=\int_{0}^{1} H_{\epsilon}(c) \eta+r H_{\epsilon}^{\prime}(c) \varphi d x \int_{0}^{1}\left(\frac{2 D_{2} c a t c^{2}}{r\left(\int_{0}^{1} r H_{\epsilon}(c)\right)^{2}}-\frac{D_{1} c}{r\left(\int_{0}^{1} r H_{\epsilon}(c) d x\right)^{2}}\right) v d x+\int_{0}^{1} \frac{2 c_{x} \eta_{x}}{r} v d x- \\
-\int_{0}^{1}\left(\frac{2 r_{x} c_{x}}{r^{2}}-\frac{2 r_{t} c}{r^{2}}-\frac{D_{1} c}{r^{2} \int_{0}^{1} r H_{\epsilon}(c) d x}\right) \eta v d x+\int_{0}^{1} \frac{2 D_{2} \delta c^{2} v}{r \int_{0}^{1} r H_{\epsilon}(c) d x} d x \forall v \in V, \text { a.e. } t \in[0, T] . \\
\eta(t, x)=\int_{0}^{t}\left(-\frac{D_{2} c a t \varphi}{\int_{0}^{1} r H_{\epsilon}(c) d x}+\frac{D_{2} c a t c}{\left(\int_{0}^{1} r H_{\epsilon}(c)\right)^{2}} \int_{0}^{1} r H_{\epsilon}^{\prime}(c) \varphi+H_{\epsilon}(c) \eta-\frac{\delta c}{\int_{0}^{1} r H_{\epsilon}(c) d x}\right) d \tau, t \in[0, T] .
\end{gathered}
$$

Similarly to Sections 3.1 and 3.2, for some given positive constant $N$, we define the set

$$
\boldsymbol{N}_{T}=\left\{(\eta, \varphi), \eta \in \mathbf{X}_{T}, \varphi \in \mathbf{Y}_{T},\|\eta\|_{\mathbf{X}_{T}}+\|\varphi\|_{\mathbf{Y}_{T}} \leq N\right\} .
$$

We present the following theorem:

Theorem 3.22 There exist $T$ small enough such that $r($ cat $) \in \mathbf{X}_{T}$ and $c($ cat $) \in \mathbf{Y}_{T}$ are Fréchet differentiable with respect to cat $\in W^{1, \infty}(\Omega)$.

In order to show that $r(c a t), c(c a t)$ are Fréchet differentiable, we will prove the following items:

1. There exist unique weak solution $(\eta, \varphi) \in N_{T}$ of the sensitivity system and they are linear, bounded with respect to $\delta \in W^{1, \infty}(\Omega)$ with $T$ mall enough. 
2. $\lim _{\|\delta\|_{W^{1, \infty}(\Omega)} \rightarrow 0} \frac{\|r(c a t+\delta)-r(c a t)-\eta\| \mathbf{x}_{T}}{\|\delta\|_{W^{1, \infty}(\Omega)}}=0$ and $\lim _{\|\delta\|_{W^{1, \infty}(\Omega)} \rightarrow 0} \frac{\|c(c a t+\delta)-c(c a t)-\varphi\|_{\mathbf{Y}_{T}}}{\|\delta\|_{W^{1, \infty}(\Omega)}}=0$

We have the following results.

Lemma 3.23 There exist solution $(\eta, \varphi) \in N_{T}$ of sensitivity system that is defined in Definition 3.20 and they are linear, bounded with respect to $\delta \in W^{1, \infty}(\Omega)$ with $T$ small enough.

Proof. We define operator

$$
\mathcal{F}: N_{T} \longrightarrow \mathbf{X}_{T} \times \mathbf{Y}_{T}
$$

as follows:

For $(\bar{\eta}, \bar{\varphi}) \in N_{T}$ define $(\eta, \varphi)=\mathcal{F}(\bar{\eta}, \bar{\varphi})$ as the solution of the system

$$
\begin{aligned}
\eta(t, x)=\int_{0}^{t}( & \left.-\frac{D_{2} c a t \varphi}{\int_{0}^{1} r H_{\epsilon}(c) d x}+\frac{D_{2} c a t c}{\left(\int_{0}^{1} r H_{\epsilon}(c)\right)^{2}} \int_{0}^{1} r H_{\epsilon}^{\prime}(c) \bar{\varphi}+H_{\epsilon}(c) \bar{\eta}-\frac{\delta c}{\int_{0}^{1} r H_{\epsilon}(c) d x}\right) d \tau, \\
& \varphi_{t}-\varphi_{x x}-\frac{r_{x} \varphi_{x}}{r}+\left(\frac{2 r_{t}}{r}+\frac{D_{1}}{r \int_{0}^{1} r H_{\epsilon}(c) d x}+\frac{2 c D_{2} c a t}{\int_{0}^{1} r H_{\epsilon}(c) d x}\right) \varphi= \\
& =\left(\frac{2 D_{2} c a t c^{2}}{r\left(\int_{0}^{1} r H_{\epsilon}(c)\right)^{2}}-\frac{D_{1} c}{r\left(\int_{0}^{1} r H_{\epsilon}(c) d x\right)^{2}}\right) \int_{0}^{1} H_{\epsilon}(c) \bar{\eta}+r H_{\epsilon}^{\prime}(c) \bar{\varphi} d x+ \\
& +\frac{2 c_{x} \eta_{x}}{r}-\left(\frac{2 r_{x} c_{x}}{r^{2}}+\frac{2 r_{t} c}{r^{2}}+\frac{D_{1} c}{r^{2} \int_{0}^{1} r H_{\epsilon}(c) d x}\right) \eta+\frac{2 D_{2} \delta c^{2}}{r \int_{0}^{1} r H_{\epsilon}(c) d x},
\end{aligned}
$$

with homogeneous initial and mixed boundary condition for $\varphi$ as written in (3.111b) - (3.111d). Similarly to Section 3.1 and Section 3.2, by applying Banach fixed point theorem, there exist unique weak solution $(\eta, \varphi) \in \boldsymbol{N}_{T}$ of the sensitivity system with $T$ small enough. Besides, as the sensitivity system is linear with respect to $c a t, \eta$ and $\varphi$, then $\eta$ and $\varphi$ are linear with respect to $\delta$. Finally, we will show that $\|\eta\|_{\mathbf{X}_{T}}$ and $\|\varphi\|_{\mathbf{Y}_{T}}$ are bounded with respect to $\|\delta\|_{W^{1, \infty}(\Omega)}$.

In one hand, apply Theorem 3.9 to the equation (3.110), we obtain

$$
\|\varphi\|_{\mathbf{Y}_{T}} \leq C\left(M, T, \epsilon, r_{\alpha}, c a t, H_{\epsilon}\right)\left(\sqrt{T}\left(\|\varphi\|_{\mathbf{Y}_{T}}+\|\eta\|_{\mathbf{x}_{T}}\right)+\|\delta\|_{L^{2}\left(Q_{T}\right)}\right) .
$$

For $T<T_{7}=\frac{1}{2 C^{2}\left(M, T, \epsilon, r_{\alpha}, c a t\right)}$, continuing (3.116)

$$
\|\varphi\|_{\mathbf{Y}_{T}} \leq C_{1}\left(M, T, \epsilon, r_{\alpha}, \text { cat }\right)\left(\sqrt{T}\|\eta\|_{\mathbf{x}_{T}}+\|\delta\|_{L^{2}\left(Q_{T}\right)}\right) .
$$

In the other hand, from (3.105), we get

$$
\|\eta\| \mathbf{x}_{T} \leq C\left(M, T, \epsilon, r_{\alpha}, \text { cat }\right) \sqrt{T}\left(\|\eta\|_{\mathbf{x}_{T}}+\|\varphi\|_{\mathbf{Y}_{T}}+\|\delta\|_{L^{\infty}\left(Q_{T}\right)}\right)
$$


Putting (3.119) into (3.120),

$$
\|\eta\| \mathbf{x}_{T} \leq C_{2}\left(M, T, \epsilon, r_{\alpha}, \text { cat }\right) \sqrt{T}\left(\|\eta\|_{\mathbf{x}_{T}}+\|\delta\|_{L^{\infty}\left(Q_{T}\right)}\right)
$$

We have, for $T<T_{8}=\frac{1}{2 C_{2}^{2}\left(M, T, \epsilon, r_{\alpha}, C a t\right)}$, the estimate

$$
\|\eta\|_{\mathbf{X}_{T}} \leq C\left(M, T, \epsilon, r_{\alpha}, c a t\right) \sqrt{T}\|\delta\|_{L^{\infty}\left(Q_{T}\right)} .
$$

Combining (3.122) and (3.119), for $T<T^{* * * *}=\min \left\{T^{* * *}, T_{7}, T_{8}\right\}$ we have

$$
\|\varphi\|_{\mathbf{Y}_{T}} \leq C\left(M, T, \epsilon, r_{\alpha}, c a t\right) \sqrt{T}\|\delta\|_{L^{\infty}\left(Q_{T}\right)}
$$

which is required to prove.

We have following Lemma:

\section{Lemma 3.24 Denote}

$$
\xi=r(c a t+\delta)-r(c a t)-\eta
$$

and

$$
\psi=c(c a t+\delta)-c(c a t)-\varphi .
$$

Then $\xi$ and $\psi$ satisfy the following system

$$
\begin{aligned}
& \psi_{t}-\psi_{x x}-\frac{2 r_{x} \psi_{x}}{r(c a t+\delta)}+F_{1}(\xi, \psi)=F_{2}(\eta, \varphi)+\omega_{\delta},(t, x) \in Q_{T}, \\
& \xi_{t}=F_{3}(\xi, \psi)+F_{4}(\eta, \varphi)+\omega_{\delta}(t, x) \in Q_{T}, \\
& \xi(0, x)=0, x \in \Omega \\
& \psi(0, x)=0, \\
& \psi(t, 0)=0, t \in[0, T] \\
& \psi_{x}(t, 0)=0,
\end{aligned}
$$

where $F_{2}(\eta, \varphi), F_{4}(\eta, \varphi)$ contain only the functions in two variables $\eta$ and $\varphi$, in which the lowestdegree term is of the second degree and $F_{1}(\xi, \psi), F_{3}(\xi, \psi)$ are linear with respect to $\xi$ and $\psi$.

$$
\left\|\omega_{\delta}\right\|_{L^{\infty}\left(Q_{T}\right)}=o\left(\|\delta\|_{W^{1, \infty}(\Omega)}\right) .
$$

Proof.

Now we aim to get a system of equations of $\xi$ and $\psi$. We see that, $(\xi, \psi)$ satisfy homogeneous initial and mix-boundary conditions:

$$
\begin{aligned}
\xi(0, x) & =0 \\
\psi(0, x) & =0 \\
\psi(t, 0) & =0 \\
\psi_{x}(t, 1) & =0
\end{aligned}
$$


Using Taylor expansion

$$
H_{\epsilon}(c(c a t+\delta))-H_{\epsilon}(c)=H_{\epsilon}^{\prime}(c)(c(c a t+\delta)-c)+\frac{1}{2} H_{\epsilon}^{\prime \prime}(c *)(c(c a t+\delta)-c)+o\left((c(c a t+\delta)-c)^{2}\right),
$$

From the Fréchet differentiability of $c \in \mathbf{Y}_{T}$ with respect to $c a t \in W^{1, \infty}(\Omega)$, we rewrite (3.127) as follows

$$
H_{\epsilon}(c(c a t+\delta))-H_{\epsilon}(c)=H_{\epsilon}^{\prime}(c)(c(c a t+\delta)-c)+\frac{1}{2} H_{\epsilon}^{\prime \prime}(c *)(c(c a t+\delta)-c)+\omega_{\delta},
$$

where

$$
\left\|\omega_{\delta}\right\|_{L^{\infty}\left(Q_{T}\right)}=o\left(\|\delta\|_{W^{1, \infty}(\Omega)}\right) .
$$

We calculate some expressions, which we will need later for obtaining a system of equations of $\xi$ and $\psi$.

$$
\begin{aligned}
I_{1} & =\frac{1}{\int_{0}^{1} r H_{\epsilon}(c) d x}-\frac{1}{\int_{0}^{1} r(c a t+\delta) H_{\epsilon}(c(c a t+\delta)) d x}= \\
= & \frac{1}{\int_{0}^{1} r H_{\epsilon}(c) d x}-\frac{1}{\int_{0}^{1} r H_{\epsilon}(c(c a t+\delta)) d x}+\frac{1}{\int_{0}^{1} r H_{\epsilon}(c(c a t+\delta)) d x}-\frac{1}{\int_{0}^{1} r(c a t+\delta) H_{\epsilon}(c(c a t+\delta)) d x}= \\
= & \frac{\int_{0}^{1}(r(c a t+\delta)-r) H_{\epsilon}(c(c a t+\delta)) d x}{\int_{0}^{1} r(c a t+\delta) H_{\epsilon}(c(c a t+\delta)) d x \int_{0}^{1} r H_{\epsilon}(c(c a t+\delta)) d x}+\frac{\int_{0}^{1} r\left(H_{\epsilon}(c(c a t+\delta))-H_{\epsilon}(c)\right) d x}{\int_{0}^{1} r H_{\epsilon}(c) d x \int_{0}^{1} r H_{\epsilon}(c(c a t+\delta)) d x}= \\
= & \frac{\int_{0}^{1} \xi H_{\epsilon}(c(c a t+\delta)) d x}{\int_{0}^{1} r(c a t+\delta) H_{\epsilon}(c(c a t+\delta)) d x \int_{0}^{1} r H_{\epsilon}(c(c a t+\delta)) d x}+\frac{\int_{0}^{1} r H_{\epsilon}^{\prime}(c) \psi d x}{\int_{0}^{1} r H_{\epsilon}(c(c a t+\delta)) d x \int_{0}^{1} r H_{\epsilon}(c) d x} \\
+ & \frac{1}{2} \frac{\int_{0}^{1} r H_{\epsilon}^{\prime \prime}(c *)(c(c a t+\delta)-c+\varphi) \psi d x}{\int_{0}^{1} r H_{\epsilon}(c(c a t+\delta)) d x \int_{0}^{1} r H_{\epsilon}(c) d x}+\frac{\int_{0}^{1} \eta H_{\epsilon}(c(c a t+\delta)) d x}{\int_{0}^{1} r(c a t+\delta) H_{\epsilon}(c(c a t+\delta)) d x \int_{0}^{1} r H_{\epsilon}(c(c a t+\delta)) d x}+ \\
+ & \frac{\int_{0}^{1} r H_{\epsilon}^{\prime}(c) \varphi d x}{\int_{0}^{1} r H_{\epsilon}(c(c a t+\delta)) d x \int_{0}^{1} r H_{\epsilon}(c) d x}+\frac{1}{2} \frac{\int_{0}^{1} r H_{\epsilon}^{\prime \prime}(c *) \varphi^{2} d x}{\int_{0}^{1} r H_{\epsilon}(c(c a t+\delta)) d x}+\omega_{\delta}=I_{1}(\xi, \psi)+I_{1}(\eta, \varphi)+\omega_{\delta}
\end{aligned}
$$

where

$$
\begin{aligned}
& I_{1}(\xi, \psi)=\frac{\int_{0}^{1} \xi H_{\epsilon}(c(c a t+\delta)) d x}{\int_{0}^{1} r(c a t+\delta) H_{\epsilon}(c(c a t+\delta)) d x \int_{0}^{1} r H_{\epsilon}(c(c a t+\delta)) d x}+\frac{\int_{0}^{1} r H^{\prime}(c) \psi d x}{\int_{0}^{1} r H_{\epsilon}(c(c a t+\delta)) d x \int_{0}^{1} r H_{\epsilon}(c) d x} \\
& +\frac{1}{2} \frac{\int_{0}^{1} r H^{\prime \prime}(c *)(c(c a t+\delta)-c+\varphi) \psi d x}{\int_{0}^{1} r H_{\epsilon}(c(c a t+\delta)) d x \int_{0}^{1} r H_{\epsilon}(c) d x}
\end{aligned}
$$


and

$$
\begin{aligned}
& I_{1}(\eta, \varphi)=\frac{\int_{0}^{1} \eta H_{\epsilon}(c(c a t+\delta)) d x}{\int_{0}^{1} r(c a t+\delta) H_{\epsilon}(c(c a t+\delta)) d x \int_{0}^{1} r H_{\epsilon}(c(c a t+\delta)) d x}+ \\
& +\frac{\int_{0}^{1} r H^{\prime}(c) \varphi d x}{\int_{0}^{1} r H_{\epsilon}(c(c a t+\delta)) d x \int_{0}^{1} r H_{\epsilon}(c) d x}+\frac{1}{2} \frac{\int_{0}^{1} r H^{\prime \prime}(c *) \varphi^{2} d x}{\int_{0}^{1} r H_{\epsilon}(c(c a t+\delta)) d x} .
\end{aligned}
$$

We see that $I_{1}(\xi, \psi)$ is linear with respect to $\xi$ or $\psi$, and $I_{1}(\eta, \varphi)$ contains only the expressions of second degree in two variable $\eta$ and $\varphi$.

We also note that

$$
\begin{aligned}
& I_{2}=\frac{1}{\int_{0}^{1} r H_{\epsilon}(c) d x}-\frac{1}{\int_{0}^{1} r(c a t+\delta) H_{\epsilon}(c(c a t+\delta)) d x}-\frac{\int_{0}^{1} r H_{\epsilon}^{\prime}(c) \varphi+H_{\epsilon}(c) \eta d x}{\left(\int_{0}^{1} r H_{\epsilon}(c) d x\right)^{2}}= \\
& =I_{1}-\frac{\int_{0}^{1} r H_{\epsilon}^{\prime}(c) \varphi+H_{\epsilon}(c) \eta d x}{\left(\int_{0}^{1} r H_{\epsilon}(c) d x\right)^{2}}=I_{1}(\xi, \psi)+I_{1}(\eta, \varphi)-\frac{\int_{0}^{1} r H_{\epsilon}^{\prime}(c) \varphi+H_{\epsilon}(c) \eta d x}{\left(\int_{0}^{1} r H_{\epsilon}(c) d x\right)^{2}}+\omega_{\delta}= \\
& =I_{1}(\xi, \psi)+\left(\frac{\int_{0}^{1} \eta H_{\epsilon}(c(c a t+\delta)) d x}{\int_{0}^{1} r(c a t+\delta) H_{\epsilon}(c(c a t+\delta)) d x \int_{0}^{1} r H_{\epsilon}(c(c a t+\delta)) d x}+\right. \\
& \left.+\frac{\int_{0}^{1} r H^{\prime}(c) \varphi d x}{\int_{0}^{1} r H_{\epsilon}(c(c a t+\delta)) d x \int_{0}^{1} r H_{\epsilon}(c) d x}+\frac{1}{2} \frac{\int_{0}^{1} r H^{\prime \prime}(c *) \varphi^{2} d x}{\int_{0}^{1} r H_{\epsilon}(c(c a t+\delta)) d x}\right)-\frac{\int_{0}^{1} r H_{\epsilon}^{\prime}(c) \varphi+H_{\epsilon}(c) \eta d x}{\left(\int_{0}^{1} r H_{\epsilon}(c) d x\right)^{2}}+\omega_{\delta}= \\
& =I_{1}(\xi, \psi)+\underbrace{\left(\frac{\int_{0}^{1} \eta H_{\epsilon}(c(c a t+\delta)) d x}{\int_{0}^{1} r(c a t+\delta) H_{\epsilon}(c(c a t+\delta)) d x \int_{0}^{1} r H_{\epsilon}(c(c a t+\delta)) d x}-\frac{\int_{0}^{1} H_{\epsilon}(c) \eta d x}{\left(\int_{0}^{1} r H_{\epsilon}(c) d x\right)^{2}}\right)}_{I_{21}}+ \\
& +\underbrace{\left(\frac{\int_{0}^{1} r H^{\prime}(c) \varphi d x}{\int_{0}^{1} r H_{\epsilon}(c(c a t+\delta)) d x \int_{0}^{1} r H_{\epsilon}(c) d x}-\frac{\int_{0}^{1} r H^{\prime}(c) \varphi d x}{\left(\int_{0}^{1} r H_{\epsilon}(c) d x\right)^{2}}\right)}_{I_{22}}+\underbrace{\frac{1}{2} \frac{\int_{0}^{1} r H^{\prime \prime}(c *) \varphi^{2} d x}{\int_{0}^{1} r H_{\epsilon}(c(c a t+\delta)) d x}}_{I_{23}(\varphi)}+\omega_{\delta},
\end{aligned}
$$

where $\omega_{\delta}$ is defined in (3.129).

Meanwhile,

$$
I_{21}=I_{21}(\xi, \psi)+I_{21}(\eta, \varphi)
$$

where

$$
I_{21}(\xi, \psi)=\frac{\int_{0}^{1} r H_{\epsilon}^{\prime}(c) \psi d x}{\int_{0}^{1} r H_{\epsilon}(c(c a t+\delta)) d x \int_{0}^{1} r H_{\epsilon}(c(c a t+\delta)) d x}+\frac{1}{2} \frac{\int_{0}^{1} \eta H_{\epsilon}^{\prime \prime}(c *)(c(c a t+\delta)-c+\varphi) \psi d x}{\int_{0}^{1} r H_{\epsilon}(c(c a t+\delta)) d x \int_{0}^{1} r H_{\epsilon}(c(c a t+\delta)) d x}-
$$




$$
\begin{aligned}
& -\frac{\int_{0}^{1} \eta H_{\epsilon}(c) d x \int_{0}^{1} H_{\epsilon}(c(c a t+\delta)) \xi d x}{\left.\left(\int_{0}^{1} r H_{\epsilon}(c(c a t+\delta)) d x\right)^{2} \int_{0}^{1} r(c a t+\delta)\right) H_{\epsilon}(c(c a t+\delta)) d x}+ \\
& +\frac{\int_{0}^{1} \eta H_{\epsilon}(c) d x \int_{0}^{1} H_{\epsilon}(c(c a t+\delta)) \xi d x}{\left(\int_{0}^{1} r H_{\epsilon}(c(c a t+\delta)) d x\right)^{2} \int_{0}^{1} r(c a t+\delta) H_{\epsilon}(c(c a t+\delta)) d x}+ \\
& +\left(\frac{\int_{0}^{1} \eta H_{\epsilon}(c) d x}{\left(\int_{0}^{1} r H_{\epsilon}(c) d x\right)^{2} \int_{0}^{1} r H_{\epsilon}(c(c a t+\delta)) d x}-\frac{\int_{0}^{1} \eta H_{\epsilon}(c) d x}{\left(\int_{0}^{1} r H_{\epsilon}(c(c a t+\delta)) d x\right)^{3}\left(\int_{0}^{1} r H_{\epsilon}(c) d x\right)^{2}}\right) \\
& \left.+\int_{0}^{1} r H_{\epsilon}^{\prime}(c) \psi d x+\frac{1}{2} \int_{0}^{1} r H^{\prime \prime}{ }_{\epsilon}(c *)(c(c a t+\delta)-c+\varphi) \psi d x\right)+ \\
& +\frac{1}{2} \frac{\int_{0}^{1} \eta H_{\epsilon}(c) d x \int_{0}^{1} r H_{\epsilon}^{\prime \prime}(c *)(c(c a t+\delta)-c+\varphi) \psi d x}{\left(\int_{0}^{1} r H_{\epsilon}(c) d x\right)^{2} \int_{0}^{1} r H_{\epsilon}(c(c a t+\delta)) d x}+\omega_{\delta}, \\
& \quad \int_{21}^{1} \eta H_{\epsilon}^{\prime}(c) \varphi d x \\
& +\frac{\int_{0}^{1} r H_{\epsilon}(c(c a t+\delta)) d x \int_{0}^{1} r H_{\epsilon}(c(c a t+\delta)) d x}{I_{0}^{2} d x} \frac{\int_{0}^{1} r H_{\epsilon}(c(c a t+\delta)) d x \int_{0}^{1} r H_{\epsilon}(c(c a t+\delta)) d x}{\left(\int_{0}^{1} r H_{\epsilon}(c(c a t+\delta)) d x\right)^{3}\left(\int_{0}^{1} r H_{\epsilon}(c) d x\right)^{2}}\left(\int_{0}^{1} r H_{\epsilon}^{\prime}(c) \varphi d x+\frac{1}{2} \int_{0}^{1} r H_{\epsilon}^{\prime \prime}(c *) \varphi^{2} d x\right)+ \\
& +\frac{\int_{0}^{1} \eta H_{\epsilon}(c) d x \int_{0}^{1} r H_{\epsilon}^{\prime}(c) \varphi d x}{\left(\int_{0}^{1} r H_{\epsilon}(c) d x\right)^{2} \int_{0}^{1} r H_{\epsilon}(c(c a t+\delta)) d x}+\omega_{\delta} .
\end{aligned}
$$

Besides,

$$
I_{22}=I_{22}(\psi)+I_{22}(\varphi),
$$

where

$I_{22}(\psi)=-\frac{\int_{0}^{1} r H_{\epsilon}^{\prime}(c) \varphi d x}{\left(\int_{0}^{1} r H_{\epsilon}(c) d x\right)^{2} \int_{0}^{1} r H_{\epsilon}(c(c a t+\delta)) d x}\left(\int_{0}^{1} r H_{\epsilon}^{\prime}(c) \psi d x+\int_{0}^{1} r H_{\epsilon}^{\prime \prime}(c *)(c(c a t+\delta)-c+\varphi) \psi d x\right)$,

$$
I_{22}(\varphi)=-\frac{\int_{0}^{1} r H_{\epsilon}^{\prime}(c) \varphi d x}{\left(\int_{0}^{1} r H_{\epsilon}(c) d x\right)^{2} \int_{0}^{1} r H_{\epsilon}(c(c a t+\delta)) d x}\left(\int_{0}^{1} r H_{\epsilon}^{\prime}(c) \varphi^{2} d x+\int_{0}^{1} r H_{\epsilon}^{\prime \prime}(c *) \varphi^{2} d x\right)+\omega_{\delta} .
$$

Denoting that

$$
I_{2}(\xi, \psi)=I_{1}(\xi, \psi)+I_{21}(\xi, \psi)+I_{22}(\psi),
$$




$$
I_{2}(\eta, \varphi)=I_{21}(\eta, \varphi)+I_{22}(\varphi)
$$

then $I_{2}=I_{2}(\xi, \psi)+I_{2}(\eta, \varphi)+\omega_{\delta}$. Note that $I_{2}(\xi, \psi)$ is linear with respect to $\xi$ or $\psi$, and $I_{2}(\eta, \varphi)$ contains the functions in two variables $\eta$ and $\varphi$, in which the lowest-degree term is of the second degree. From (3.100), (3.101) and (3.105), we have

$$
\begin{aligned}
r_{t}(c a t+\delta)- & r_{t}-\eta_{t}=-\frac{D_{2} c a t \psi}{\int_{0}^{1} r(c a t+\delta) H_{\epsilon}(c(c a t+\delta)) d x}-\frac{D_{2} \delta \psi}{\int_{0}^{1} r(c a t+\delta) H_{\epsilon}(c(c a t+\delta)) d x}+ \\
& +\left(D_{2} c a t \varphi-D_{2} \delta c\right)(\underbrace{\left(\frac{1}{\int_{0}^{1} r H_{\epsilon}(c) d x}-\frac{1}{\int_{0}^{1} r(c a t+\delta) H_{\epsilon}(c(c a t+\delta)) d x}\right)}_{I_{1}}+ \\
& +D_{2} \operatorname{cat} c \underbrace{\left(\frac{1}{\int_{0}^{1} r H_{\epsilon}(c) d x}-\frac{1}{\int_{0}^{1} r(c a t+\delta) H_{\epsilon}(c(c a t+\delta)) d x}-\frac{\int_{0}^{1} r H_{\epsilon}^{\prime}(c) \varphi+H_{\epsilon}(c) \eta d x}{\left(\int_{0}^{1} r H_{\epsilon}(c) d x\right)^{2}}\right)}_{I_{2}}+\omega_{\delta} .
\end{aligned}
$$

Combining (3.122) with the above equalities

$$
\begin{aligned}
& \xi_{t}=-\frac{D_{2} c a t \psi}{\int_{0}^{1} r(c a t+\delta) H_{\epsilon}(c(c a t+\delta)) d x}+\frac{D_{2} \delta \psi}{\int_{0}^{1} r(c a t+\delta) H_{\epsilon}(c(c a t+\delta)) d x}+\left(D_{2} c a t \varphi-D_{2} \delta c\right) I_{1}(\xi, \psi)+ \\
& +D_{2} \operatorname{catc}\left(I_{1}(\xi, \psi)+I_{21}(\xi, \psi)+I_{22}(\xi, \psi)\right)+\left(D_{2} \operatorname{cat} \varphi-D_{2} \delta c\right) I_{1}(\eta, \varphi)+D_{2} \operatorname{catc}\left(I_{21}(\eta, \varphi)+I_{22}(\eta, \varphi)+I_{3}(\varphi)\right)+\omega_{\delta} .
\end{aligned}
$$

Denote

$$
\begin{aligned}
& F_{3}(\xi, \psi)=-\frac{D_{2} c a t \psi}{\int_{0}^{1} r(c a t+\delta) H_{\epsilon}(c(c a t+\delta)) d x}+\frac{D_{2} \delta \psi}{\int_{0}^{1} r(c a t+\delta) H_{\epsilon}(c(c a t+\delta)) d x}+\left(D_{2} c a t \varphi-D_{2} \delta c\right) I_{1}(\xi, \psi)+ \\
& +D_{2} \operatorname{catc}\left(I_{1}(\xi, \psi)+I_{21}(\xi, \psi)+I_{22}(\xi, \psi)\right),
\end{aligned}
$$

$$
F_{4}(\eta, \varphi)=\left(D_{2} \operatorname{cat} \varphi-D_{2} \delta c\right) I_{1}(\eta, \varphi)+D_{2} \operatorname{cat} c\left(I_{21}(\eta, \varphi)+I_{22}(\eta, \varphi)+I_{3}(\varphi)\right),
$$

we get (3.124b). We notice that $F_{3}(\xi, \psi)$ is linear with respect to $\xi$ and $\psi, F_{4}(\eta, \varphi)$ contains only the functions in two variables $\eta$ and $\varphi$, in which the lowest-degree term is of the second degree. Due to Lemma 3.23, $\eta \in \mathbf{X}_{T}$ and $\varphi \in \mathbf{Y}_{T}$ are linear bounded with respect to $\delta \in W^{1, \infty}(\Omega)$ with some $T$ small enough. Therefore,

$$
\frac{\left\|F_{4}(\eta, \varphi)\right\|_{L^{2}(Q)}}{\|\delta\|_{W^{1, \infty}(\Omega)}}=o\left(\|\delta\|_{W^{1, \infty}(\Omega)}\right)
$$


Substracting (3.106) by (3.107)

$\left(c_{t}(c a t+\delta)-c_{t}\right)-\left(c_{x x}(c a t+\delta)-c_{x x}\right)-\left(\frac{2 r_{x}(c a t+\delta) c_{x}(c a t+\delta)}{r(c a t+\delta)}-\frac{2 r_{t} c}{r}\right)+$ $+\left(\frac{\left(2 r_{t}(c a t+\delta) c(c a t+\delta)\right.}{r(c a t+\delta)}-\frac{2 r_{t} c}{r}\right)+\left(\frac{D_{1} c(c a t+\delta)}{r(c a t+\delta) \int_{0}^{1} r(c a t+\delta) H_{\epsilon}(c(c a t+\delta)) d x}-\frac{D_{1} c}{r \int_{0}^{1} r H_{\epsilon}(c) d x},\right)=0$

which is equivalent to

$$
\begin{aligned}
& \left(c_{t}(c a t+\delta)-c_{t}\right)-\left(c_{x x}(c a t+\delta)-c_{x x}\right)-\frac{2\left(r_{x}(c a t+\delta)-r_{x}\right) c_{x}(c a t+\delta)}{r(c a t+\delta)}-\frac{2 r_{x}\left(c_{x}(c a t+\delta)-c_{x}\right)}{r(c a t+\delta)}+ \\
& +\frac{2 r_{x} c_{x}(r(c a t+\delta)-r)}{r r(c a t+\delta)}+\frac{2 r_{t}(c a t+\delta)(c(c a t+\delta)-c)}{r(c a t+\delta)}+\frac{2\left(r_{t}(c a t+\delta)-r_{t}\right) c}{r(c a t+\delta)}-\frac{2 r_{t} c(r(c a t+\delta)-r)}{r r(c a t+\delta)}+ \\
& +\frac{D_{1}(c(c a t+\delta)-c)}{r(c a t+\delta) \int_{0}^{1} r(c a t+\delta) H_{\epsilon}(c(c a t+\delta)) d x}-\frac{D_{1} c(r(c a t+\delta)-r)}{r r(c a t+\delta) \int_{0}^{1} r(c a t+\delta) H_{\epsilon}(c(c a t+\delta)) d x}- \\
& -\frac{D_{1} c \int_{0}^{1}(r(c a t+\delta)-r) H_{\epsilon}(c(c a t+\delta)) d x}{r \int_{0}^{1} r(c a t+\delta) H_{\epsilon}(c(c a t+\delta)) d x \int_{0}^{1} r H_{\epsilon}(c(c a t+\delta)) d x}-\frac{D_{1} c \int_{0}^{1} r\left(H_{\epsilon}(c(c a t+\delta))-H_{\epsilon}(c)\right) d x}{r \int_{0}^{1} r H_{\epsilon}(c(c a t+\delta)) d x \int_{0}^{1} r H_{\epsilon}(c) d x}=0 .
\end{aligned}
$$

Now substracting (3.109) by (3.146)

$$
\begin{aligned}
& \left(c_{t}(c a t+\delta)-c_{t}-\varphi_{t}\right)-\left(c_{x x}(c a t+\delta)-c_{x x}-\varphi_{x x}\right)-\underbrace{\left.\frac{2\left(r_{x}(c a t+\delta)-r_{x}\right) c_{x}(c a t+\delta)}{r(c a t+\delta)}-\frac{2 c_{x} \eta_{x}}{r}\right)}_{G_{1}}- \\
& -\underbrace{\left(\frac{2 r_{x}\left(c_{x}(c a t+\delta)-c_{x}\right)}{r(c a t+\delta)}-\frac{2 r_{x} \varphi_{x}}{r}\right)}_{G_{2}}+\underbrace{\left(\frac{2 r_{x} c_{x}(r(c a t+\delta)-r)}{r r(c a t+\delta)}-\frac{2 r_{x} c_{x} \eta}{r^{2}}\right)}_{G_{3}}+ \\
& +\underbrace{\left(\frac{2 r_{t}(c a t+\delta)(c(c a t+\delta)-c)}{r(c a t+\delta)}-\frac{2 r_{t} \varphi}{r}\right)}_{G_{4}}+\underbrace{\left(\frac{2\left(r_{t}(c a t+\delta)-r_{t}\right) c}{r(c a t+\delta)}-\frac{2 c \eta_{t}}{r}\right)}_{G_{5}}- \\
& -\underbrace{\left(\frac{2 r_{t} c(r(c a t+\delta)-r)}{r r(c a t+\delta)}-\frac{2 r_{t} c \eta}{r^{2}}\right)}_{G_{6}}+\underbrace{\left(\frac{D_{1}(c(c a t+\delta)-c)}{r(c a t+\delta) \int_{0}^{1} r(c a t+\delta) H_{\epsilon}(c(c a t+\delta)) d x}-\frac{D_{1} \varphi}{r \int_{0}^{1} r H_{\epsilon}(c) d x}\right)}_{G_{7}}- \\
& -\underbrace{\left(\frac{D_{1} c(r(c a t+\delta)-r)}{r r(c a t+\delta) \int_{0}^{1} r(c a t+\delta) H_{\epsilon}(c(c a t+\delta)) d x}-\frac{D_{1} c \eta}{r^{2} \int_{0}^{1} r H_{\epsilon}(c) d x}\right)}_{G_{8}}- \\
& -\underbrace{\left(\frac{D_{1} c \int_{0}^{1} r H_{\epsilon}^{\prime}(c)(c(c a t+\delta)-c) d x}{r \int_{0}^{1} r H_{\epsilon}(c(c a t+\delta)) d x \int_{0}^{1} r H_{\epsilon}(c) d x}-\frac{D_{1} c \int_{0}^{1} r H_{\epsilon}^{\prime}(c) \varphi d x}{r\left(\int_{0}^{1} r H_{\epsilon}(c) d x\right)^{2}}\right)}_{G_{9}}-
\end{aligned}
$$




$$
\begin{aligned}
& -\frac{1}{2} \underbrace{\frac{D_{1} c \int_{0}^{1} r H_{\epsilon}^{\prime \prime}(c *)(c(c a t+\delta)-c)^{2} d x}{r \int_{0}^{1} r H_{\epsilon}(c(c a t+\delta)) d x \int_{0}^{1} r H_{\epsilon}(c) d x}}_{G_{10}}- \\
& -\underbrace{\left(\frac{D_{1} c \int_{0}^{1}(r(c a t+\delta)-r) H_{\epsilon}(c) d x}{r \int_{0}^{1} r(c a t+\delta) H_{\epsilon}(c(c a t+\delta)) d x \int_{0}^{1} r H_{\epsilon}(c(c a t+\delta)) d x}-\frac{D_{1} c \int_{0}^{1} r H_{\epsilon}(c) \eta d x}{r\left(\int_{0}^{1} r H_{\epsilon}(c) d x\right)^{2}}\right)}_{G_{11}}- \\
& -\underbrace{\frac{D_{1} c \int_{0}^{1}(r(c a t+\delta)-r) H_{\epsilon}^{\prime}(c)(c(c a t+\delta)-c) d x}{r \int_{0}^{1} r(c a t+\delta) H_{\epsilon}(c(c a t+\delta)) d x \int_{0}^{1} r H_{\epsilon}(c(c a t+\delta)) d x}}_{G_{12}}- \\
& -\underbrace{\frac{D_{1} c \int_{0}^{1}(r(c a t+\delta)-r) H_{\epsilon}^{\prime \prime}(c *)(c(c a t+\delta)-c)^{2} d x}{r \int_{0}^{1} r(c a t+\delta) H_{\epsilon}(c(c a t+\delta)) d x \int_{0}^{1} r H_{\epsilon}(c(c a t+\delta)) d x}}_{G_{13}}+\omega_{\delta}=0 .
\end{aligned}
$$

Now we analysize $G_{1}-G_{13}$ :

$$
\begin{gathered}
G_{1}=\frac{2 \xi c_{x}(c a t+\delta)}{r(c a t+\delta)}+\frac{2 \eta_{x} \psi}{r(c a t+\delta)}-\frac{2 \eta_{x} c_{x} \xi}{r r(c a t+\delta)}+\frac{2 \eta_{x} \varphi}{r(c a t+\delta)}-\frac{2 \eta_{x} c_{x} \eta}{r r(c a t+\delta)}=G_{1}(\xi, \psi)+G_{1}(\eta, \varphi) \\
G_{2}=\frac{2 r_{x} \psi_{x}}{r(c a t+\delta)}-\frac{2 r_{x} \varphi_{x} \xi}{r r(c a t+\delta)}-\frac{2 r_{x} \varphi_{x} \eta}{r r(c a t+\delta)}=G_{2}(\xi, \psi)+G_{2}(\eta, \varphi) \\
G_{3}=\frac{2 r_{x} c_{x} \psi}{r r(c a t+\delta)}-\frac{2 r_{x} \eta c_{x} \xi}{r^{2} r(c a t+\delta)}-\frac{2 r_{x} c_{x} \eta^{2}}{r^{2} r(c a t+\delta)}=G_{3}(\xi, \psi)+G_{3}(\eta, \varphi) \\
G_{4}=\frac{2 r_{t} \psi}{r(c a t+\delta)}+\frac{2 \varphi \xi_{t}}{r(c a t+\delta)}+\frac{2 \varphi \eta_{t}}{r(c a t+\delta)}-\frac{2 r_{t} \varphi \xi}{r r(c a t+\delta)}-\frac{2 r_{t} \varphi \eta}{r r(c a t+\delta)}
\end{gathered}
$$

Putting (3.124b) into (3.151), we get

$$
\begin{gathered}
G_{4}=\frac{2 r_{t} \psi}{r(c a t+\delta)}+\frac{2 \varphi\left(F_{3}(\xi, \psi)+F_{4}(\eta, \varphi)+\omega_{\delta}\right)}{r(c a t+\delta)}+\frac{2 \varphi \eta_{t}}{r(c a t+\delta)}-\frac{2 r_{t} \varphi \xi}{r r(c a t+\delta)}-\frac{2 r_{t} \varphi \eta}{r r(c a t+\delta)}=G_{2}(\xi, \psi)+G_{2}(\eta, \varphi) . \\
G_{5}=\frac{2 c \xi_{t}}{r(c a t+\delta)}-\frac{2 c \eta_{t} \xi}{r r(c a t+\delta)}-\frac{2 c \eta_{t} \eta}{r r(c a t+\delta)}=G_{5}(\xi, \psi)+G_{5}(\eta, \varphi) .
\end{gathered}
$$


Putting (3.124b) into (3.153), we have

$$
\begin{aligned}
& G_{5}=\frac{2 c\left(F_{3}(\xi, \psi)+F_{4}(\eta, \varphi)+\omega_{\delta}\right)}{r(c a t+\delta)}-\frac{2 c \eta_{t} \xi}{r r(c a t+\delta)}-\frac{2 c \eta_{t} \eta}{r r(c a t+\delta)}=G_{5}(\xi, \psi)+G_{5}(\eta, \varphi) . \\
& G_{7}=\frac{2 r_{t} c \xi}{r r(c a t+\delta)}-\frac{2 r_{t} c \eta \xi}{r^{2} r(c a t+\delta)}-\frac{2 r_{t} c \eta^{2}}{r^{2} r(c a t+\delta)}=G_{6}(\xi, \psi)+G_{6}(\eta, \varphi) . \\
& \\
&-\frac{D_{1} \psi}{r(c a t+\delta) \int_{0}^{1} r(c a t+\delta) H_{\epsilon}(c(c a t+\delta)) d x}-\frac{D_{1} \varphi \xi}{r(c a t+\delta) \int_{0}^{1} r(c a t+\delta) H_{\epsilon}(c(c a t+\delta)) d x}- \\
&-\frac{D_{1} \varphi \int_{0}^{1} \xi H_{\epsilon}(c(c a t+\delta)) d x}{r \int_{0}^{1} r(c a t+\delta) H_{\epsilon}(c(c a t+\delta)) d x \int_{0}^{1} r H_{\epsilon}(c(c a t+\delta)) d x}- \\
&-\frac{1}{2} \frac{D_{1} \varphi \int_{0}^{1} r H_{\epsilon}^{\prime}(c) \psi d x}{r \int_{0}^{1} r(c a t+\delta) H_{\epsilon}(c(c a t+\delta)) d x \int_{0}^{1} r H_{\epsilon}(c(c a t+\delta)) d x}- \\
&-\frac{D_{1} \varphi \int_{0}^{1} r H_{\epsilon}^{\prime \prime}(c *)(c(c a t+\delta)-c+\varphi) \psi(c) H_{\epsilon}(c(c a t+\delta)) d x \int_{0}^{1} r H_{\epsilon}(c(c a t+\delta)) d x}{r r(c a t+\delta) \int_{0}^{1} r(c a t+\delta) H_{\epsilon}(c(c a t+\delta)) d x}-\frac{D_{1} \varphi \int_{0}^{1} r(c a t+\delta) H_{\epsilon}(c(c a t+\delta)) d x \int_{0}^{1} r H_{\epsilon}(c(c a t+\delta)) d x}{D_{1} \varphi \int_{0}^{1} r H_{\epsilon}^{\prime}(c) \varphi d x}- \\
&-\frac{D_{1} \varphi \int_{0}^{1} r H_{\epsilon}^{\prime \prime}(c *) \varphi^{2} d x}{r \int_{0}^{1} r H_{\epsilon}(c(c a t+\delta)) d x \int_{0}^{1} r H_{\epsilon}(c) d x}-\frac{1}{2} \frac{D_{1} \int_{0}^{1} r H_{\epsilon}(c(c a t+\delta)) d x \int_{0}^{1} r H_{\epsilon}(c) d x}{\eta H_{\epsilon}(c(c a t+\delta)) d x}-G_{7}(\xi)+G_{7}(\eta, \varphi) .
\end{aligned}
$$

$$
\begin{aligned}
G_{8}= & \frac{D_{1} c \xi}{r r(c a t+\delta) \int_{0}^{1} r(c a t+\delta) H_{\epsilon}(c(c a t+\delta)) d x}-\frac{D_{1} c \eta \xi}{r^{2} r(c a t+\delta) \int_{0}^{1} r(c a t+\delta) H_{\epsilon}(c(c a t+\delta)) d x}- \\
& -\frac{D_{1} c \eta \int_{0}^{1} \xi H_{\epsilon}(c(c a t+\delta)) d x}{r^{2} \int_{0}^{1} r(c a t+\delta) H_{\epsilon}(c(c a t+\delta)) d x \int_{0}^{1} r H_{\epsilon}(c(c a t+\delta)) d x}-\frac{D_{1} c \eta \int_{0}^{1} r H_{\epsilon}^{\prime}(c) \psi d x}{r \int_{0}^{1} r H_{\epsilon}(c(c a t+\delta)) d x \int_{0}^{1} r H_{\epsilon}(c) d x}- \\
& -\frac{1}{2} \frac{D_{1} c \eta \int_{0}^{1} r H_{\epsilon}^{\prime \prime}(c *)(c(c a t+\delta)-c+\varphi) \psi d x}{r \int_{0}^{1} r H_{\epsilon}(c(c a t+\delta)) d x \int_{0}^{1} r H_{\epsilon}(c) d x}-\frac{D_{1} c \eta^{2}}{r^{2} r(c a t+\delta) \int_{0}^{1} r(c a t+\delta) H_{\epsilon}(c(c a t+\delta)) d x}- \\
& -\frac{D_{1} c \eta \int_{0}^{1} \eta H_{\epsilon}(c(c a t+\delta)) d x}{r^{2} \int_{0}^{1} r(c a t+\delta) H_{\epsilon}(c(c a t+\delta)) d x \int_{0}^{1} r H_{\epsilon}(c(c a t+\delta)) d x}-\frac{D_{1} c \eta \int_{0}^{1} r H_{\epsilon}^{\prime}(c) \varphi d x}{r^{2} \int_{0}^{1} r H_{\epsilon}(c(c a t+\delta)) d x \int_{0}^{1} r H_{\epsilon}(c) d x}- \\
& -\frac{1}{2} \frac{D_{1} c \eta \int_{0}^{1} r H_{\epsilon}^{\prime \prime}(c *) \varphi^{2} d x}{r \int_{0}^{1} r^{2} H_{\epsilon}(c(c a t+\delta)) d x \int_{0}^{1} r H_{\epsilon}(c) d x}=G_{8}(\xi, \psi)+G_{8}(\eta, \varphi) .
\end{aligned}
$$




$$
\begin{aligned}
& G_{9}=\frac{D_{1} c \int_{0}^{1} r H_{\epsilon}^{\prime}(c) \psi d x}{r \int_{0}^{1} r H_{\epsilon}(c(c a t+\delta)) d x \int_{0}^{1} r H_{\epsilon}(c) d x}-\frac{D_{1} c \int_{0}^{1} r H_{\epsilon}^{\prime}(c) \varphi d x \int_{0}^{1} r H_{\epsilon}^{\prime}(c) \psi d x}{r \int_{0}^{1} r H_{\epsilon}(c(c a t+\delta)) d x\left(\int_{0}^{1} r H_{\epsilon}(c) d x\right)^{2}}- \\
& -\frac{1}{2} \frac{D_{1} c \int_{0}^{1} r H_{\epsilon}^{\prime}(c) \varphi d x \int_{0}^{1} r H_{\epsilon}^{\prime \prime}(c *)(c(c a t+\delta)-c+\varphi) \psi d x}{r \int_{0}^{1} r H_{\epsilon}(c(c a t+\delta)) d x\left(\int_{0}^{1} r H_{\epsilon}(c) d x\right)^{2}}- \\
& -\frac{D_{1} c\left(\int_{0}^{1} r H_{\epsilon}^{\prime}(c) \varphi d x\right)^{2}}{r \int_{0}^{1} r H_{\epsilon}(c(c a t+\delta)) d x\left(\int_{0}^{1} r H_{\epsilon}(c) d x\right)^{2}}-\frac{1}{2} \frac{D_{1} c \int_{0}^{1} r H_{\epsilon}^{\prime}(c) \varphi d x \int_{0}^{1} r H_{\epsilon}^{\prime \prime}(c *) \varphi^{2} d x}{r \int_{0}^{1} r H_{\epsilon}(c(c a t+\delta)) d x\left(\int_{0}^{1} r H_{\epsilon}(c) d x\right)^{2}}= \\
& =G_{9}(\xi, \psi)+G_{9}(\eta, \varphi) .
\end{aligned}
$$

$$
\begin{aligned}
& G_{10}=-\frac{1}{2} \frac{D_{1} c \int_{0}^{1} r H_{\epsilon}^{\prime \prime}(c *)(c(c a t+\delta)-c+\varphi) \psi d x}{r\left(\int_{0}^{1} r H_{\epsilon}(c(c a t+\delta)) d x\right)^{2}}-\frac{1}{2} \frac{D_{1} c \int_{0}^{1} r H_{\epsilon}^{\prime \prime}(c *) \varphi^{2} d x}{r\left(\int_{0}^{1} r H_{\epsilon}(c(c a t+\delta)) d x\right)^{2}}= \\
& =G_{10}(\xi, \psi)+G_{10}(\eta, \varphi) .
\end{aligned}
$$

$$
\begin{aligned}
G_{11}= & \frac{D_{1} c \int_{0}^{1} H_{\epsilon}(c) \xi d x}{r \int_{0}^{1} r(c a t+\delta) H_{\epsilon}(c(c a t+\delta)) d x \int_{0}^{1} r H_{\epsilon}(c(c a t+\delta)) d x}- \\
& -\frac{D_{1} c \int_{0}^{1} \eta H_{\epsilon}(c) d x \int_{0}^{1} r H_{\epsilon}^{\prime}(c) \psi d x}{r \int_{0}^{1} r(c a t+\delta) H_{\epsilon}(c(c a t+\delta)) d x \int_{0}^{1} r H_{\epsilon}(c(c a t+\delta)) d x \int_{0}^{1} r H_{\epsilon}(c) d x}- \\
& -\frac{1}{2} \frac{D_{1} c \int_{0}^{1} \eta H_{\epsilon}(c) d x \int_{0}^{1} r H_{\epsilon}^{\prime \prime}(c *)(c(c a t+\delta)-c+\varphi) \psi d x}{r \int_{0}^{1} r(c a t+\delta) H_{\epsilon}(c(c a t+\delta)) d x \int_{0}^{1} r H_{\epsilon}(c(c a t+\delta)) d x \int_{0}^{1} r H_{\epsilon}(c) d x}- \\
& -\frac{D_{1} c \int_{0}^{1} \eta H_{\epsilon}(c) d x \int_{0}^{1} H_{\epsilon}(c(c a t+\delta)) \xi d x}{r \int_{0}^{1} r(c a t+\delta) H_{\epsilon}(c(c a t+\delta)) d x \int_{0}^{1} r H_{\epsilon}(c(c a t+\delta)) d x \int_{0}^{1} r H_{\epsilon}(c) d x}- \\
& -\frac{D_{1} c \int_{0}^{1} \eta H_{\epsilon}(c) d x \int_{0}^{1} r H_{\epsilon}^{\prime}(c) \psi d x}{r \int_{0}^{1} r H_{\epsilon}(c(c a t+\delta)) d x\left(\int_{0}^{1} r H_{\epsilon}(c) d x\right)^{2}}-\frac{1}{2} \frac{D_{1} c \int_{0}^{1} \eta H_{\epsilon}(c) d x \int_{0}^{1} r H_{\epsilon}^{\prime \prime}(c *)(c(c a t+\delta)-c+\varphi) \psi d x}{r \int_{0}^{1} r H_{\epsilon}(c(c a t+\delta)) d x\left(\int_{0}^{1} r H_{\epsilon}(c) d x\right)^{2}}- \\
& -\frac{D_{1} c \int_{0}^{1} \eta H_{\epsilon}(c) d x \int_{0}^{1} r H_{\epsilon}^{\prime}(c) \varphi d x}{r \int_{0}^{1} r(c a t+\delta) H_{\epsilon}(c(c a t+\delta)) d x \int_{0}^{1} r H_{\epsilon}(c(c a t+\delta)) d x \int_{0}^{1} r H_{\epsilon}(c) d x}- \\
& -\frac{1}{2} \frac{D_{1} c \int_{0}^{1} \eta H_{\epsilon}(c) d x \int_{0}^{1} r H_{\epsilon}^{\prime \prime}(c *) \varphi^{2} d x}{r \int_{0}^{1} r(c a t+\delta) H_{\epsilon}(c(c a t+\delta)) d x \int_{0}^{1} r H_{\epsilon}(c(c a t+\delta)) d x \int_{0}^{1} r H_{\epsilon}(c) d x}- \\
& -\frac{D_{1} c \int_{0}^{1} \eta H_{\epsilon}(c) d x \int_{0}^{1} H_{\epsilon}(c(c a t+\delta)) \varphi d x}{r \int_{0}^{1} r(c a t+\delta) H_{\epsilon}(c(c a t+\delta)) d x \int_{0}^{1} r H_{\epsilon}(c(c a t+\delta)) d x \int_{0}^{1} r H_{\epsilon}(c) d x}-
\end{aligned}
$$




$$
\begin{aligned}
& -\frac{D_{1} c \int_{0}^{1} \eta H_{\epsilon}(c) d x \int_{0}^{1} r H_{\epsilon}^{\prime}(c) \varphi d x}{r \int_{0}^{1} r H_{\epsilon}(c(c a t+\delta)) d x\left(\int_{0}^{1} r H_{\epsilon}(c) d x\right)^{2}}-\frac{1}{2} \frac{D_{1} c \int_{0}^{1} \eta H_{\epsilon}(c) d x \int_{0}^{1} r H_{\epsilon}^{\prime \prime}(c *) \varphi^{2} d x}{r \int_{0}^{1} r H_{\epsilon}(c(c a t+\delta)) d x\left(\int_{0}^{1} r H_{\epsilon}(c) d x\right)^{2}}= \\
& =G_{11}(\xi, \psi)+G_{11}(\eta, \varphi) .
\end{aligned}
$$

$$
\begin{aligned}
& G_{12}=-\frac{D_{1} c \int_{0}^{1} \xi H_{\epsilon}^{\prime}(c)(c(c a t+\delta)-c) d x}{r \int_{0}^{1} r(c a t+\delta) H_{\epsilon}(c(c a t+\delta)) d x \int_{0}^{1} r H_{\epsilon}(c(c a t+\delta)) d x}+ \\
&+\frac{D_{1} c \int_{0}^{1} \eta H_{\epsilon}^{\prime}(c) \psi d x}{r \int_{0}^{1} r(c a t+\delta) H_{\epsilon}(c(c a t+\delta)) d x \int_{0}^{1} r H_{\epsilon}(c(c a t+\delta)) d x}+ \\
&+\frac{D_{1} c \int_{0}^{1} \eta H_{\epsilon}^{\prime}(c) \varphi d x}{r \int_{0}^{1} r(c a t+\delta) H_{\epsilon}(c(c a t+\delta)) d x \int_{0}^{1} r H_{\epsilon}(c(c a t+\delta)) d x}=G_{12}(\xi, \psi)+G_{12}(\eta, \varphi) . \\
& G_{13}=\frac{1}{2} \frac{D_{1} c \int_{0}^{1} \xi H_{\epsilon}^{\prime \prime}(c *)(c(c a t+\delta)-c)^{2} d x}{r \int_{0}^{1} r(c a t+\delta) H_{\epsilon}(c(c a t+\delta)) d x \int_{0}^{1} r H_{\epsilon}(c(c a t+\delta)) d x}+ \\
&+ \frac{1}{2} \frac{D_{1} c \int_{0}^{1} \eta H_{\epsilon}^{\prime \prime}(c *)(c(c a t+\delta)-c+\varphi) \psi d x}{r \int_{0}^{1} r(c a t+\delta) H_{\epsilon}(c(c a t+\delta)) d x \int_{0}^{1} r H_{\epsilon}(c(c a t+\delta)) d x}+ \\
&+\frac{1}{2} \frac{D_{1} c \int_{0}^{1} \eta H_{\epsilon}^{\prime \prime}(c *) \varphi^{2} d x}{r \int_{0}^{1} r(c a t+\delta) H_{\epsilon}(c(c a t+\delta)) d x \int_{0}^{1} r H_{\epsilon}(c(c a t+\delta)) d x}=G_{13}(\xi, \psi)+G_{13}(\eta, \varphi) .
\end{aligned}
$$

Combine (3.147) and the equalities from (3.148) - (3.159), we deduce

$$
\psi_{t}-\psi_{x x}-\frac{2 r_{x} \psi_{x}}{r(c a t+\delta)}+\sum_{i=1}^{13} G_{i}(\xi, \psi)=-\sum_{i=1}^{13} G_{i}(\eta, \varphi)+\omega_{\delta},
$$

where $F_{1}(\xi, \psi)=\sum_{i=1}^{13} G_{i}(\xi, \psi)$ is linear with respect to $\psi, \xi_{x}, \xi$ and the coefficients $F_{2}(\eta, \varphi)=$ $\sum_{i=1}^{13} G_{i}(\eta, \varphi)$ contain only the functions in variables $\eta, \varphi, \eta_{x}$ and $\varphi_{x}$ in which the lowest-degree term is of the second degree. Moreover, for the sake of Lemma 3.23, $\eta \in \mathbf{X}_{T}$ and $\varphi \in \mathbf{Y}_{T}$ are linear bounded with respect to $\delta \in W^{1, \infty}(\Omega)$ with some $T$ small enough. Hence, it satisfies that

$$
\frac{\left\|F_{2}(\eta, \varphi)\right\|_{L^{2}(Q)}}{\|\delta\|_{W^{1, \infty}(\Omega)}}=o\left(\|\delta\|_{W^{1, \infty}(\Omega)}\right) .
$$

The lemma now follows from (3.141), (3.143), (3.142), (3.163) and (3.126).

We define a weak solution of system (3.124a) - (3.124f) in the following sense. 
Definition 3.25 A pair $(\xi, \psi) \in W^{1, \infty}\left(Q_{T}\right) \times W\left(0, T ; H^{1}(\Omega)\right)$ is said to be a weak solution of the the system (3.124a) - (3.124f) if the following equalities are satisfied

$$
\begin{gathered}
\left(\psi_{t}, v\right)_{\left(\left(H^{1}(\Omega)\right)^{*}, H^{1}(\Omega)\right)}+\int_{0}^{1} \psi_{x} v_{x} d x-\int_{0}^{1} \frac{2 r_{x} \psi_{x}}{r(c a t+\delta)} v d x+\int_{0}^{1} F_{1}(\xi, \psi) v d x= \\
=\int_{0}^{1}\left(F_{2}(\eta, \varphi)+\omega_{\delta}\right) v d x \forall v \in V \text {, a.e. } t \in[0, T] . \\
\psi(0, x)=0, x \in[0,1] . \\
\xi(t, x)=\int_{0}^{t} F_{3}(\xi, \psi)+F_{4}(\eta, \varphi)+\omega_{\delta} d \tau, t \in[0, T] .
\end{gathered}
$$

Let $N^{\xi, \psi}$ be some positive constant, denote the set

$$
\boldsymbol{N}_{T}^{\xi, \psi}=\left\{(\xi, \psi), \xi \in \mathbf{X}_{T}, \psi \in \mathbf{Y}_{T},\|\xi\|_{\mathbf{X}_{T}}+\|\psi\|_{\mathbf{Y}_{T}} \leq N^{\xi, \psi}\right\}
$$

We have the following result:

Theorem 3.26 There exist enough small positive number $T$ such that the system (3.165) - (3.167) has unique solution on $\boldsymbol{N}_{T}^{\xi, \psi}$ and

(i) $\lim _{\|\delta\|_{W^{1, \infty}(\Omega)} \rightarrow 0} \frac{\|\xi\|_{\mathbf{X}_{T}}}{\|\delta\|_{W^{1, \infty}(\Omega)}}=0$

(ii) $\lim _{\|\delta\|_{W^{1, \infty}(\Omega)} \rightarrow 0} \frac{\|\psi\|_{\mathbf{Y}_{T}}}{\|\delta\|_{W^{1, \infty}(\Omega)}}=0$.

Proof. We define the operator

$$
\mathcal{K}: N_{T}^{\xi, \psi} \longrightarrow \mathbf{X}_{T} \times \mathbf{Y}_{T}
$$

as follows.

For $(\bar{\xi}, \bar{\psi}) \in \boldsymbol{N}_{T}^{\xi, \psi}$ define $(\xi, \psi)=\mathcal{K}(\bar{\xi}, \bar{\psi})$ as the solution of the system

$$
\begin{gathered}
\xi(t, x)=\int_{0}^{t}\left(F_{3}(\bar{\xi}, \bar{\psi})+F_{4}(\eta, \varphi)+\omega_{\delta}\right) d \tau, \\
\psi_{t}-\psi_{x x}-\frac{2 r_{x} \psi_{x}}{r(c a t+\delta)}+F_{1}(\xi, \psi)=F_{2}(\eta, \varphi)+\omega_{\delta},
\end{gathered}
$$

with homogeneous initial and mixed boundary condition for $\psi$ as written in (3.124d) - (3.124f). Similarly to Section 3.1 and Section 3.2, by applying Banach fixed point theorem, there exist unique weak solution $(\xi, \psi) \in N_{T}^{\xi, \psi}$ of the system (3.124a) - (3.124f) with $T$ small enough. Using the similar 
technique in Section 3.1 and Section 3.2 and using Gronwall's inequality, for $T$ small enough, we have the following estimates:

$$
\|\xi\|_{\mathbf{X}_{T}}+\|\psi\|_{\mathbf{Y}_{T}} \leq C=C\left(M, T, \epsilon, r_{\alpha}, c a t\right)\left(\left\|F_{2}(\eta, \varphi)+\omega_{\delta}\right\|_{L^{2}\left(0, T ; L^{2}(\Omega)\right)}+\left\|F_{4}(\eta, \varphi)+\omega_{\delta}\right\|_{L^{2}\left(0, T ; L^{2}(\Omega)\right)}\right) .
$$

The Theorem now follows from (3.144), (3.164) and (3.171).

\subsection{Formulation and weak solution of problem $I_{b}$}

Now we consider the case of cat depending on the both variables $x$ and $t$.

$$
\begin{gathered}
c_{t}=c_{x x}+\frac{2 r_{x} c_{x}}{r}-\frac{\left(2 r_{t}+\frac{D_{1}}{\int_{0}^{1} r H_{\epsilon}(c) d x}\right) c}{r},(t, x) \in Q_{T} . \\
r_{t}=-\frac{D_{2}}{\int_{0}^{1} r H_{\epsilon}(u+g) d x} \operatorname{cat}(x) c,(t, x) \in Q_{T}, \\
\text { cat }_{t}=\alpha_{0} \int_{0}^{1} H_{R_{0}}(r(y, t)) G(x-y) d y,
\end{gathered}
$$

where $\alpha_{0}$ is some positive number. with the initial and mixed boundary conditions

$$
\begin{gathered}
r(0, x)=r_{\alpha}(x)=1, x \in \Omega, \\
c(0, x)=h(x), \\
c(t, 0)=1, t \in[0, T], \\
c_{x}(t, 1)=0, t \in[0, T], \\
\operatorname{cat}(0, x)=\operatorname{cat}_{0}(x)
\end{gathered}
$$

We suppose the conditions on $h$ and cat $_{0}$ by

$$
\begin{array}{r}
h(0)=0, h(1)=0, \\
h \in W^{1, \infty}(\Omega), \\
\operatorname{cat}_{0} \in W^{1, \infty}(\Omega) .
\end{array}
$$


The functions $\beta(t, r, c)$ and $\gamma(t, r, c)$ are kept as in (3.8), where $H_{\epsilon}(x)$ is from (A.12). Using the similar change of variables of $w(t, x)=c(t, x)-1$ in Problem $I$ (system of (3.172)-(3.174)) and conditions (3.3)-(3.6), we deduce the new equivalent system

$$
\begin{gathered}
w_{t}-w_{x x}-\frac{2 r_{x} w_{x}}{r}+\left(\frac{2 r_{t}}{r}+\frac{D_{1}-2 D_{2} \operatorname{cat}(t, x)}{r \int_{0}^{1} r H_{\epsilon}(w+1) d x}\right) w=\frac{2 D_{2} \operatorname{cat}(t, x)-D_{1}}{r \int_{0}^{1} r H_{\epsilon}(w+1) d x},(t, x) \in Q_{T}, \\
r_{t}=-\frac{D_{2}}{\int_{0}^{1} r H_{\epsilon}(w+g) d x} \operatorname{cat}(t, x)(w+1),(t, x) \in Q_{T}, \\
\operatorname{cat}_{t}=\alpha_{0} \int_{0}^{1} H_{R_{0}}(r(y, t)) G(x-y) d y, \\
r(0, x)=r_{\alpha}(x)=1, x \in \Omega, \\
w(0, x)=w_{0}(x)=h(0)-1, x \in \Omega, \\
w_{x}(t, 1)=0, t \in[0, T], \\
\operatorname{cat}(0, x)=\operatorname{cat}_{0}(x) .
\end{gathered}
$$

The solution of this system is understood in the following sense:

Definition 3.27 The pair $(r, w) \in W^{1, \infty}\left(Q_{T}\right) \times W\left(0, T ; H^{1}(\Omega)\right)$ is said to be a weak solution of (3.177)(3.181), (3.3), if the following equalities are satisfied

$$
\begin{array}{r}
\left(w_{t}, v\right)_{\left(\left(H^{1}(\Omega)\right)^{*}, H^{1}(\Omega)\right)}+\int_{0}^{1} w_{x} v_{x} d x-\int_{0}^{1} \frac{2 r_{x} w_{x}}{r} v d x+\int_{0}^{1}\left(\frac{2 r_{t}}{r}+\frac{D_{1}-2 D_{2} \operatorname{cat}(t, x)}{r \int_{0}^{1} r H_{\epsilon}(w+1) d x}\right) u v d x= \\
=\int_{0}^{1} \frac{2 D_{2} \operatorname{cat}(t, x)-D_{1}}{r \int_{0}^{1} r H_{\epsilon}(w+1) d x} v d x, \\
\forall v \in V, \text { and with a.e.t } \in[0, T]
\end{array}
$$

where $V=\left\{v \in H^{1}(\Omega), v(0)=0\right\}$,

$$
w(0, x)=w_{0}(x)=h(0)-1, x \in \Omega,
$$




$$
r(t, x)=1-\int_{0}^{t} \frac{D_{2}}{\int_{0}^{1} r H_{\epsilon}(u+g) d x} \operatorname{cat}(t, x)(u(\tau, x)+1) d \tau, t \in[0, T],
$$

where

$$
\operatorname{cat}(t, x)=\operatorname{cat}_{0}(x)+\alpha_{0} \int_{0}^{t} \int_{0}^{1} H_{R_{0}}(r(y, t)) G(x-y) d y .
$$

\subsection{Existence and uniqueness of the weak solution of problem $I_{b}$ with cat $(t, x)$ model}

Recalling the spaces $\mathbf{X}_{T}, \mathbf{Y}_{T}$ and set $\boldsymbol{M}_{T} \in \mathbf{X}_{T} \times \mathbf{Y}_{T}$ in the page 34, we define an operator $\mathcal{S}$

$$
\mathcal{S}: \boldsymbol{M}_{T} \longrightarrow \mathbf{X}_{T} \times \mathbf{Y}_{T}
$$

For $(\bar{r}, \bar{w}) \in \boldsymbol{M}_{T}$ define $(r, w)=\mathcal{S}(\bar{r}, \bar{w})$ as the solution of following system of the equations

$$
\begin{gathered}
\operatorname{cat}(t, x)=\operatorname{cat}_{0}(x)+\alpha_{0} \int_{0}^{t} \int_{0}^{1} H_{R_{0}}(\bar{r}(t, y)) G(x-y) d y, \\
r(t, x)=1-\int_{0}^{t} \frac{D_{2}}{\int_{0}^{1} \bar{r} H_{\epsilon}(\bar{w}+1) d x} \operatorname{cat}(t, x)(\bar{w}+1) d \tau, \\
w_{t}+\mathscr{L} w=f,
\end{gathered}
$$

with the initial and boundary condition (3.16), (3.17), where

$$
\begin{array}{r}
\mathscr{L} w=-w_{x x}-\frac{2 r_{x} w_{x}}{r}+\left(\frac{2 r_{t}}{r}+\frac{D_{1}-2 D_{2} \operatorname{cat}(x)}{r \int_{0}^{1} r H_{\epsilon}(\bar{w}+1) d x}\right) w, \\
f=\frac{2 D_{2} \operatorname{cat}(x)-D_{1}}{r \int_{0}^{1} r H_{\epsilon}(\bar{w}+1) d x} .
\end{array}
$$

We follow the similar approach for proving problem $I_{a}$ to deal with the problem $I_{b}$ :

1) $\mathcal{S}$ is defined from $\boldsymbol{M}_{T}$ onto $\mathbf{X}_{T} \times \mathbf{Y}_{T}$.

2) For $T$ small enough such that $\mathcal{S}$ maps $\boldsymbol{M}_{T}$ to $\boldsymbol{M}_{T}$.

3) For $T$ small enough such that $\mathcal{S}$ is s-contractive in $\mathbf{X}_{T} \times \mathbf{Y}_{T}$, i.e

$$
\left\|\mathcal{S}\left(w_{1}, r_{1}\right)-\mathcal{S}\left(w_{2}, r_{2}\right)\right\|_{\mathbf{X}_{T} \times \mathbf{Y}_{T}} \leq s^{*}\left\|\left(w_{1}, r_{1}\right)-\left(w_{2}, r_{2}\right)\right\|_{\mathbf{X}_{T} \times \mathbf{Y}_{T}},
$$

for all $\left(w_{1}, r_{1}\right),\left(w_{2}, r_{2}\right) \in \boldsymbol{M}_{T}$, and for $0 \leq s^{*}<1$.

We have 
Remark 3.28 Under condition on cat $t_{0}(x)$ in (3.176), cat $(t, x)$ from equation (3.174) belongs to space $W^{1, \infty}(\Omega)$.

Thanks to above remark, we can reply the whole steps of the proof in section 3.2 for the existence and uniqueness of the weak solution of the problem $I_{a}$.

\subsection{Sensitivity system for the Problem $I_{b}$}

Suppose that $r, c, c a t$ are the solution of Problem $I_{b}$ with the initial condition on cat is $\operatorname{cat}(0, x)=$ cat $_{0}$, and $r\left(c a t_{0}+\mu \delta\right), c\left(c a t_{0}+\mu \delta\right), c a t\left(c a t_{0}+\mu \delta\right)$ are the solution of $I_{b}$ with $c a t\left(c a t_{0}+\mu \delta, 0, x\right)=c_{a t}+\mu \delta$. Here $\delta \in W^{1, \infty}(\Omega)$.

Denote

$$
\begin{aligned}
& \eta(t, x):=\frac{\partial r}{\partial c a t_{0}}\left(c a t_{0}\right) \delta=\lim _{\mu \rightarrow 0} \frac{r\left(c a t_{0}+\mu \delta\right)-r}{\mu}, \\
& \varphi(t, x):=\frac{\partial c}{\partial c a t_{0}}\left(c a t_{0}\right) \delta=\lim _{\mu \rightarrow 0} \frac{c\left(c^{2 a t_{0}}+\mu \delta\right)-c}{\mu}, \\
& \psi(t, x):=\frac{\partial c a t}{\partial c a t_{0}}\left(c_{a t}\right) \delta=\lim _{\mu \rightarrow 0} \frac{\operatorname{cat}\left(c a t_{0}+\mu \delta\right)-\operatorname{cat}\left(c a t_{0}\right)}{\mu} .
\end{aligned}
$$

Using the same technique in section 3.4, the sensitivity system for problem $I_{b}$ is given by

$$
\begin{gathered}
\eta_{t}(t, x)=-\frac{D_{2} c \psi}{\int_{0}^{1} r H_{\epsilon}(c) d x}-\frac{D_{2} c a t_{0} \varphi}{\int_{0}^{1} r H_{\epsilon}(c) d x}+\frac{D_{2} c a t_{0} c}{\left(\int_{0}^{1} r H_{\epsilon}(c)\right)^{2}} \int_{0}^{1} r H_{\epsilon}^{\prime}(c) \varphi+H_{\epsilon}(c) \eta-\frac{\delta c}{\int_{0}^{1} r H_{\epsilon}(c) d x}, \\
\varphi_{t}-\varphi_{x x}-\frac{r_{x} \varphi_{x}}{r}+\left(\frac{2 r_{t}}{r}+\frac{D_{1}}{r \int_{0}^{1} r H_{\epsilon}(c) d x}+\frac{D_{2} c a t}{\int_{0}^{1} r H_{\epsilon}(c) d x}\right) \varphi= \\
=-\left(\frac{D_{1} c}{r\left(\int_{0}^{1} r H_{\epsilon}(c) d x\right)^{2}}-\frac{2 D_{2} c a t c^{2}}{r\left(\int_{0}^{1} r H_{\epsilon}(c)\right)^{2}}\right) \int_{0}^{1} H_{\epsilon}(c) \bar{\eta}+r H_{\epsilon}^{\prime}(c) \bar{\varphi} d x+ \\
+\frac{2 c_{x} \eta_{x}}{r}-\left(\frac{2 r_{x} c_{x}}{r^{2}}+\frac{2 r_{t} c}{r^{2}}+\frac{D_{1} c}{r^{2} \int_{0}^{1} r H_{\epsilon}(c) d x}\right) \eta-\frac{2 \delta c^{2}}{r \int_{0}^{1} r H_{\epsilon}(c) d x}
\end{gathered}
$$

and

$$
\psi_{t}(t, x)=\int_{0}^{1} H_{R_{0}}^{\prime}\left(c a t_{0} ; y, t\right) \eta(y, t) G(x-y) d y
$$


with the initial conditions and boundary conditions

$$
\begin{aligned}
& \eta(0, x)=0, \\
& \varphi(0, x)=0, \\
& \varphi(t, 0)=0, \\
& \varphi_{x}(t, 1)=0, \\
& \psi(0, x)=\delta .
\end{aligned}
$$

Denote $\zeta(t, x)=\psi(t, x)-\delta(x),(3.190),(3.192)$ and (3.197) become

$$
\begin{gathered}
\eta_{t}(t, x)=-\frac{D_{2} c \zeta}{\int_{0}^{1} r H_{\epsilon}(c) d x}-\frac{D_{2} c \delta}{\int_{0}^{1} r H_{\epsilon}(c) d x}-\frac{D_{2} c a t \varphi}{\int_{0}^{1} r H_{\epsilon}(c) d x}+\frac{D_{2} c a t c}{\left(\int_{0}^{1} r H_{\epsilon}(c)\right)^{2}} \int_{0}^{1} r H_{\epsilon}^{\prime}(c) \varphi+H_{\epsilon}(c) \eta-\frac{\delta c}{\int_{0}^{1} r H_{\epsilon}(c) d x}, \\
\left.\zeta_{t}(t, x)=\int_{0}^{1} H_{R_{0}}^{\prime}\left(c a t_{0} ; y, t\right) \eta(y, t) G(x-y) d y\right) \\
\zeta(0, x)=0 .
\end{gathered}
$$

Remark 3.29 For clarity, we use notation $H_{R_{0}}\left(c a t_{0} ; y, t\right)$ for the function $H_{R_{0}}(r(t, y))$ to note that $r(y, t)=r\left(c a t_{0} ; y, t\right)$.

In order to show that $r\left(c_{a t}\right), c\left(c a t_{0}\right)$ are Fréchet differentiable with respect to $c t_{0}$, we will prove that there exist $T$ small enough such that the following items hold:

1. There exist solution $\eta, \varphi, \zeta$ of system of the equations (3.191), (3.198) and (3.199) with homogeneous initial condition, boundary conditions (3.193)-(3.196), (3.200) and they are linear, bounded with respect to $\delta$.

2. $\lim _{\|\delta\|_{W^{1, \infty}(\Omega)} \rightarrow 0} \frac{\left\|r\left(c a t_{0}+\delta\right)-r\left(c a t_{0}\right)-\eta\right\|_{\mathbf{x}_{T}}}{\|\delta\|_{W^{1, \infty}(\Omega)}}=0$ and $\lim _{\|\delta\|_{W^{1, \infty}(\Omega)} \rightarrow 0} \frac{\left\|c\left(c a t_{0}+\delta\right)-c\left(c a t_{0}\right)-\varphi\right\|_{\mathbf{Y}_{T}}}{\|\delta\|_{W^{1}, \infty}(\Omega)}=0$.

For this, we present the following results. 
Lemma 3.30 There exist solution $\eta, \varphi, \zeta$ of system of the equations (3.191), (3.198) and (3.199) with homogeneous initial condition, boundary conditions (3.193)-(3.196), (3.200) and they are linear, bounded with respect to $\delta$.

Proof. Relying on the proof of Lemma 3.23, Using the similar technique in the sections 3.1 and 3.2, with $N_{t}$ is some given positive constant we define the set

$$
\boldsymbol{N}_{T}=\left\{(\eta, \varphi), \eta \in \mathbf{X}_{T}, \varphi \in \mathbf{Y}_{T},\|\eta\|_{\mathbf{X}_{T}}+\|\varphi\|_{\mathbf{Y}_{T}} \leq N\right\} .
$$

And we also define an operator $z$

$$
z: N_{t} \longrightarrow \mathbf{X}_{T} \times \mathbf{Y}_{T}
$$

by the following approach: For $(\bar{\eta}, \bar{\varphi}) \in N_{t}$ define $(\eta, \varphi)=\mathcal{Z}(\bar{\eta}, \bar{\varphi})$ as the solution of following system of the equation

$$
\begin{aligned}
\eta(t, x)=\int_{0}^{t}( & \left.-\frac{D_{2} c a t \varphi}{\int_{0}^{1} r H_{\epsilon}(c) d x}+\frac{D_{2} c a t c}{\left(\int_{0}^{1} r H_{\epsilon}(c)\right)^{2}} \int_{0}^{1} r H_{\epsilon}^{\prime}(c) \bar{\varphi}+H_{\epsilon}(c) \bar{\eta}-\frac{\delta c}{\int_{0}^{1} r H_{\epsilon}(c) d x}\right) d \tau, \\
& \varphi_{t}-\varphi_{x x}-\frac{r_{x} \varphi_{x}}{r}+\left(\frac{2 r_{t}}{r}+\frac{D_{1}}{r \int_{0}^{1} r H_{\epsilon}(c) d x}+\frac{D_{2} c a t}{\int_{0}^{1} r H_{\epsilon}(c) d x}\right) \varphi= \\
= & -\left(\frac{D_{1} c}{r\left(\int_{0}^{1} r H_{\epsilon}(c) d x\right)^{2}}-\frac{2 D_{2} c a t c^{2}}{r\left(\int_{0}^{1} r H_{\epsilon}(c)\right)^{2}}\right) \int_{0}^{1} H_{\epsilon}(c) \bar{\eta}+r H_{\epsilon}^{\prime}(c) \bar{\varphi} d x+ \\
& +\frac{2 c_{x} \eta_{x}}{r}-\left(\frac{2 r_{x} c_{x}}{r^{2}}+\frac{2 r_{t} c}{r^{2}}+\frac{D_{1} c}{r^{2} \int_{0}^{1} r H_{\epsilon}(c) d x}\right) \eta-\frac{2 \delta c^{2}}{r \int_{0}^{1} r H_{\epsilon}(c) d x},
\end{aligned}
$$

where

$$
\zeta(t, x)=\int_{0}^{t} \int_{0}^{1} H_{R_{0}}^{\prime}\left(y, \tau, c a t_{0}\right) \bar{\eta}(y, t) G(x-y) d y d \tau .
$$

The initial condition and boundary conditions on the equations (3.202) and (3.203) remain homogeneous like in (3.193)-(3.196).

Following the idea from Lemma 3.23, we finish the proof of this Lemma.

With the same technique in Subsection 3.4.1, we have the following Lemma and Theorem.

Lemma 3.31 Let $\eta, \varphi$ be the solution of system of the equations (3.191), (3.198) and (3.199) with homogeneous initial condition, boundary conditions (3.193)-(3.196), (3.200), then

- $\lim _{\|\delta\|_{W^{1, \infty}(\Omega)} \rightarrow 0} \frac{\left\|r\left(c a t_{0}+\delta\right)-r\left(c a t_{0}\right)-\eta\right\|_{\mathbf{X}_{T}}}{\|\delta\|_{W^{1}, \infty}(\Omega)}=0$. 
- $\lim _{\|\delta\|_{W^{1, \infty}(\Omega)} \rightarrow 0} \frac{\left\|c\left(c a t_{0}+\delta\right)-c\left(c a t_{0}\right)-\varphi\right\|_{\mathbf{Y}_{T}}}{\|\delta\|_{W^{1, \infty}(\Omega)}}=0$.

Theorem 3.32 There exist $T$ small enough such that $r\left(\right.$ cat $\left._{0}\right) \in \mathbf{X}_{T}$ and $c\left(\right.$ cat $\left._{0}\right) \in \mathbf{Y}_{T}$ are Frechet differentiable with respect to cat $t_{0} \in W^{1, \infty}(\Omega)$.

\subsection{Formulation and weak solution of the problem $I I_{a}$}

The problem $I I$ defined in definition 1.1 with $\operatorname{cat}(x)$ catalyst positioning Model after reformulating the two coefficients $\gamma(r, c), \beta(r, c)$ leads to the below system

$$
\begin{gathered}
c_{t}-r^{\frac{2}{3}} c_{x x}+\frac{2 r_{x} c_{x}}{r^{\frac{1}{3}}}-\frac{\left(\frac{4}{3} r_{t}+\frac{D_{1}}{\int_{0}^{1} r^{\frac{1}{3}} H_{\epsilon}(c) d x}\right) c}{r}=0,(t, x) \in Q_{T}, \\
r_{t}=-\frac{D_{2}}{\int_{0}^{1} r^{\frac{1}{3}} H_{\epsilon}(c) d x} \operatorname{cat}(x) c,(t, x) \in Q_{T},
\end{gathered}
$$

with the initial and boundary conditions (3.4), (3.12), (3.6):

$$
c(0, x)=h(x),
$$

and mixed boundary conditions

$$
\begin{gathered}
c(t, 0)=1, t \in[0, T], \\
c_{x}(t, 1)=0, t \in[0, T] .
\end{gathered}
$$

The conditions on $h(x), \operatorname{cat}(x)$ are the same in (3.7)

$$
\begin{aligned}
& h(0)=1, h(1)=0, \\
& h \in W^{2, \infty}(\Omega), \\
& \text { cat } \in W^{1 \infty}(\Omega),
\end{aligned}
$$

and $H_{\epsilon}(x)$ is defined in (3.9). By setting

$$
\mathbf{u}(t, x)=c(t, x)-1,
$$

we have $\mathbf{u}(t, 0)=\mathbf{u}_{x}(t, 1)=0$. We also denote $\mathbf{u}_{0}(x)=h(x)-1$. The equation (3.212) leads to

$$
\mathbf{u}_{t}-r^{\frac{2}{3}} \mathbf{u}_{x x}-\frac{2 r_{x} \mathbf{u}_{x}}{r^{\frac{1}{3}}}+\frac{\left(\frac{4}{3} r_{t}+\frac{D_{1}}{\int_{0}^{1} r^{\frac{1}{3}} H_{\epsilon}(\mathbf{u}+1) d x}\right) \mathbf{u}}{r}=-\frac{\left(\frac{4}{3} r_{t}+\frac{D_{1}}{\int_{0}^{1} r^{\frac{1}{3}} H_{\epsilon}(\mathbf{u}+1) d x}\right)}{r},(t, x) \in Q_{T} .
$$


Putting $r_{t}=-\frac{D_{2}}{\int_{0}^{1} r^{\frac{1}{3}} H_{\epsilon}(\mathbf{u}+1) d x} \operatorname{cat}(x)(\mathbf{u}+1),(t, x) \in Q_{T}$ into the right hand side of (3.212), then we have the system

$$
\begin{gathered}
\mathbf{u}_{t}-r^{\frac{2}{3}} \mathbf{u}_{x x}-\frac{2 r_{x} \mathbf{u}_{x}}{r}+\left(\frac{\frac{4}{3} r_{t}}{r}+\frac{D_{1}-\frac{4}{3} D_{2} \operatorname{cat}(x)}{r \int_{0}^{1} r^{\frac{1}{3}} H_{\epsilon}(\mathbf{u}+1) d x}\right) \mathbf{u}=\frac{\frac{4}{3} D_{2} \operatorname{cat}(x)-D_{1}}{r \int_{0}^{1} r^{\frac{1}{3}} H_{\epsilon}(\mathbf{u}+1) d x},(t, x) \in Q_{T}, \\
r_{t}=-\frac{D_{2}}{\int_{0}^{1} r^{\frac{1}{3}} H_{\epsilon}(\mathbf{u}+1) d x} \operatorname{cat}(x)(\mathbf{u}+1),(t, x) \in Q_{T}, \\
r(0, x)=r_{0}(x)=1, x \in \Omega, \\
\mathbf{u}(0, x)=u_{0}(x), x \in \Omega, \\
\mathbf{u}_{x}(t, 1)=0, t \in[0, T] .
\end{gathered}
$$

We give the definition of a weak solution.

Definition 3.33 The pair $(r, \mathbf{u}) \in W^{1, \infty}\left(Q_{T}\right) \times W\left(0, T ; H^{1}(\Omega)\right)$ is said to be a weak solution of (3.213)(3.217) if the following equalities are satisfied

$$
\begin{array}{r}
\left(\mathbf{u}_{t}, v\right)_{\left(\left(H^{1}(\Omega)\right)^{*}, H^{1}(\Omega)\right)}+\int_{0}^{1} r^{\frac{2}{3}} \mathbf{u}_{x} x v d x-\int_{0}^{1} \frac{2 r_{x} \mathbf{u}_{x}}{r} v d x+\int_{0}^{1}\left(\frac{\frac{4}{3} r_{t}}{r}+\frac{D_{1}-\frac{4}{3} D_{2} \operatorname{cat}(x)}{r \int_{0}^{1} r^{\frac{1}{3}} H_{\epsilon}(\mathbf{u}+1) d x}\right) \mathbf{u} v d x= \\
=\int_{0}^{1} \frac{\frac{4}{3} D_{2} \operatorname{cat}(x)-D_{1}}{r \int_{0}^{1} r^{\frac{1}{3}} H_{\epsilon}(\mathbf{u}+1) d x} v d x, \\
\forall v \in V, \text { and with a.e.t } \in[0, T],
\end{array}
$$

where

$$
\begin{gathered}
V=\left\{v \in H^{1}(\Omega), v(0)=0\right\}, \\
\mathbf{u}(0, x)=\mathbf{u}_{0}(x), x \in[0,1], \\
r(t, x)=1-\int_{0}^{t} \frac{D_{2}}{\int_{0}^{1} r H_{\epsilon}(\mathbf{u}+1) d x} \operatorname{cat}(x)(\mathbf{u}(\tau, x)+1) d \tau, t \in[0, T] .
\end{gathered}
$$


Relying on the basic results on regularity of parabolic equation for initial mixed boundary condition from Chapter 2, the fixed point theory, following the Sections 3.1-3.7, we can conclude the wellposedness of the weak solution of Problem $I I_{a}$ and $I I_{b}$, the Frechet differentiability $r_{c a t}$ or $r_{c a t_{0}}$. 



\section{Chapter 4}

\section{Optimization Problems}

In this Chapter we investigate mainly the two optimization problems, which subject to $I_{a}$ and $I_{b}$. The optimization problems subject to $I I_{a}$ and $I I_{b}$ are considered in similar way.

From Chapter 3, we know that for Problems $I_{a}$ and $I I_{a}(\operatorname{cat}(x)$ Model), there exist continuous operator:

$$
\begin{aligned}
& \mathcal{F}_{1}: W^{1, \infty}(\Omega) \longrightarrow \mathbf{X}_{T} \times \mathbf{Y}_{T} \\
& \quad c a t \longmapsto(r(c a t)(t, x), c(c a t)(t, x)) .
\end{aligned}
$$

For Problems $I_{b}$ and $I_{b}(c a t(t, x)$ Model $)$, there exist continuous operator:

$$
\begin{aligned}
\mathcal{F}_{2}: W^{1, \infty}(\Omega) & \longrightarrow \mathbf{X}_{T} \times \mathbf{Y}_{T} \\
\quad c a t_{0} & \longmapsto\left(r\left(c a t_{0}\right)(t, x), c\left(c a t_{0}\right)(t, x)\right) .
\end{aligned}
$$

We have the following remark.

Remark 4.1 For convenience, we will use notations:

(i) $r(c a t), c(c a t)$ or $r(c a t ; t, x), c(c a t ; t, x)$ for $r(c a t)(t, x), c(c a t)(t, x)$ in cat $(x)$ Model.

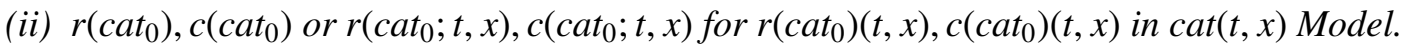

alternatively in relevant situations.

\subsection{The first optimization problem}

We consider the first optimization problem for the $\operatorname{cat}(x)$ model, the $\operatorname{cat}(t, x)$ case can be treated similarly.

$$
\min _{c a t \in A} V(c a t)=\min _{c a t \in A} \int_{0}^{1} r^{2}(c a t)(T, x) d x .
$$

subjects to Problem $I_{a}$ and $I I_{a}$. 


\subsubsection{Frechet differentiability of the objective functional with respect to catalytic func- tion}

As $r \in \mathbf{X}_{T} \subset C\left(0, T ; L^{2}(\Omega)\right)$, we can define the linear bounded projection operator

$$
\begin{aligned}
\mathbf{P}_{T}: \mathbf{X}_{T} & \longrightarrow L^{2}(\Omega) \\
r(c a t ; t, .) & \longmapsto r(c a t ; T, .) .
\end{aligned}
$$

The first optimization problem is to minimize the following objective functional

$$
V(c a t)=\int_{0}^{1} r^{2}(c a t)(T, x) d x=\int_{0}^{1}\left(\mathbf{P}_{T} r(c a t)(x)\right)^{2} d x .
$$

We know from Chapter 3 that $r(c a t)$ is Fréchet differentiable with respect to $c a t \in W^{1, \infty}(\Omega)$, i.e. there exist a linear operator

$$
\begin{aligned}
& r_{\text {cat }}: W^{1, \infty}(\Omega) \longrightarrow L\left(W^{1, \infty}(\Omega), \mathbf{X}_{T}\right) \\
& \text { cat } \longmapsto r_{\text {cat }}(\text { cat }),
\end{aligned}
$$

such that

$$
\left\|r(c a t+\delta)-r(c a t)-r_{c a t}(c a t) \delta\right\| \mathbf{x}_{T}=o\left(\|\delta\|_{W^{1, \infty}(\Omega)}\right) .
$$

Remark 4.2 We use the abbreviation $r_{\text {cat }}$ for $r_{\text {cat }}($.$) like in Chapter 3$.

Theorem 4.3 Functional $V$ in (4.2) is Fréchet differentiable with respect to cat $\in W^{1, \infty(\Omega)}$.

Proof. Taking $\delta \in W^{1, \infty}(\Omega)$, denoting by $(\cdot, \cdot)$ the inner product in the Hilbert space $L^{2}(\Omega)$, we calculate

$$
\begin{array}{r}
V(c a t+\delta)-V(c a t)=\left\|\mathbf{P}_{T} r(c a t+\delta)\right\|_{L^{2}(\Omega)}^{2}-\left\|\mathbf{P}_{T} r(c a t)\right\|_{L^{2}(\Omega)}^{2}= \\
=\left\|\mathbf{P}_{T} r(c a t+\delta)-\mathbf{P}_{T} r(c a t)+\mathbf{P}_{T} r(c a t)\right\|_{L^{2}(\Omega)}^{2}-\left\|\mathbf{P}_{T} r(c a t)\right\|_{L^{2}(\Omega)}^{2}= \\
=2\left(\mathbf{P}_{T}(r(c a t+\delta)-r(c a t)), \mathbf{P}_{T} r(c a t)\right)+\left\|\mathbf{P}_{T}(r(c a t+\delta)-r(c a t))\right\|_{L^{2}(\Omega)}^{2} .
\end{array}
$$

We see that

$\left(\mathbf{P}_{T}(r(c a t+\delta)-r(c a t)), \mathbf{P}_{T} r(c a t)\right)-\left(\mathbf{P}_{T} r_{c a t} \delta, \mathbf{P}_{T} r(c a t)\right)=\left(\mathbf{P}_{T}(r(c a t+\delta)-r(c a t))-\mathbf{P}_{T} r_{c a t} \delta, \mathbf{P}_{T} r(c a t)\right) \leq$ $\leq\left\|\mathbf{P}_{T}(r(c a t+\delta)-r(c a t))-\mathbf{P}_{T} r_{c a t} \delta\right\|_{L^{2}(\Omega)}\left\|\mathbf{P}_{T} r(c a t)\right\|_{L^{2}(\Omega)} \leq\left\|r(c a t+\delta)-r(c a t)-r_{c a t} \delta\right\|_{L^{2}(\Omega)}\left\|r_{c a t} \delta\right\|_{L^{2}(\Omega)}$. 
Combine (4.4) and (4.6), we have:

$$
\left(\mathbf{P}_{T}(r(c a t+\delta)-r(c a t)), \mathbf{P}_{T} r(c a t)\right)=\left(\mathbf{P}_{T} r_{c a t} \delta, \mathbf{P}_{T} r(c a t)\right)+o\left(\|\delta\|_{W^{1, \infty}(\Omega)}\right) .
$$

We continue (4.5), using (4.7) and Theorem about continuity of $(r, c)$ in $W^{1, \infty}\left(Q_{T}\right) \times \mathbf{Y}_{T}$ with respect to cat in $W^{1, \infty}(\Omega)$ as follows

$$
V(c a t+\delta)-V(c a t)=2\left(\mathbf{P}_{T} r_{c a t} \delta, \mathbf{P}_{T} r(c a t)\right)+o\left(\|\delta\|_{W^{1, \infty}(\Omega)}\right) .
$$

Hence, we can define the linear bounded operator

$$
\begin{aligned}
V_{c a t}: W^{1, \infty}(\Omega) & \longrightarrow L\left(W^{1, \infty}(\Omega), \mathbb{R}\right) \\
\text { cat } & \longmapsto V_{c a t}(c a t)=2\left(\mathbf{P}_{T} r_{c a t}, \mathbf{P}_{T} r(c a t)\right) .
\end{aligned}
$$

Remark 4.4 For convenience, we use the abbreviation $V_{\text {cat }}$ for $V_{\text {cat }}($.$) from now on.$

\subsubsection{The existence of the solution of the first optimization problem}

Now we prove the the existence of a minimizer of the functional(4.2) over an admissible set. The idea to choose this admissible set we learn from the papers of Dinh-Nho Hào et al [11] and Rosch et al [31]. We introduce the admissible set $\boldsymbol{A}$ as follows

$$
\boldsymbol{A}=\left\{\operatorname{cat} \in C^{1, \lambda}(\Omega), 0 \leq c a t \leq 1, M_{1} \leq \operatorname{cat}_{x} \leq M_{2}, \forall x \in[0,1], \sup _{x_{1}, x_{2} \in[0,1]} \frac{\left|\operatorname{cat}_{x}\left(x_{1}\right)-\operatorname{cat}_{x}\left(x_{2}\right)\right|}{\left|x_{1}-x_{2}\right|^{\lambda}} \leq C\right\},
$$

where $M_{1}, M_{2}$ are some given positive numbers and $0<\lambda<1$. The set $\boldsymbol{A}$ is compact in $C^{1}(\Omega)$ (see [31]). Since $V($ cat $)$ is continuous on $W^{1, \infty}(\Omega)$, therefore we have the following results.

Theorem 4.5 The problem of minimizing V(cat) subjects to Problem $I_{a}$ (or $I I_{a}$ ) over $\boldsymbol{A}$ admits at least one solution.

For the first optimization problem subjects to Problem $I_{b}$ and $I I_{b}$, we define another admissible set as follows:

$\boldsymbol{A}_{c a t_{0}}=\left\{\operatorname{cat}_{0} \in C^{1, \lambda}(\Omega), 0 \leq \operatorname{cat}_{0} \leq 1, M_{1} \leq \operatorname{cat}_{0 x} \leq M_{2}, \forall x \in[0,1], \sup _{x_{1}, x_{2} \in[0,1]} \frac{\left|\operatorname{cat}_{0 x}\left(x_{1}\right)-\operatorname{cat}_{0 x}\left(x_{2}\right)\right|}{\left|x_{1}-x_{2}\right|^{\lambda}} \leq C\right\}$, 
Theorem 4.6 For the first optimization problem subjects to Problem $I_{b}$ and $I I_{b}$, we replace the admission set $\boldsymbol{A}_{\text {cat }}$ from (4.10) by the set $\boldsymbol{A}_{\text {cat }}$ defined in (4.11). Then this problem of minimizing $V(c a t)$ over $\boldsymbol{A}_{\text {cat }_{0}}$ admits at least one solution.

For the proofs of Theorem 4.5 and 4.6, see [11] and [31].

Suppose that in the $\operatorname{cat}(x)$ model, there exist the linear bounded operators:

$$
\begin{aligned}
r_{c a t}: L^{2}(\Omega) & \longrightarrow L^{2}\left(Q_{T}\right) \\
\delta & \longmapsto r_{c a t} \delta, \\
c_{c a t}: L^{2}(\Omega) & \longrightarrow L^{2}\left(Q_{T}\right) \\
\delta & \longmapsto c_{c a t} \delta
\end{aligned}
$$

and suppose that the projection operator $\mathbf{P}_{T}$ is bounded from $L^{2}\left(Q_{T}\right)$ onto $L^{2}(\Omega)$

$$
\begin{aligned}
\mathbf{P}_{T}: L^{2}\left(Q_{T}\right) & \longrightarrow L^{2}(\Omega) \\
r(c a t ; t, x) & \longmapsto r(c a t ; T, x) .
\end{aligned}
$$

We get the following theorem:

Theorem 4.7 Under assumptions (4.12) - (4.14), the Fréchet derivative $V_{c a t}$ in the cat $(x)$ model (Problem $\left.I_{a}, I I_{a}\right)$ is defined by $2\left(r_{c a t}\right)^{*}\left(\mathbf{P}_{T}\right)^{*} \mathbf{P}_{T} r($ cat $)$.

Proof. The proof is a result of Theorem 4.3 and the assumptions (4.12) - (4.14). - The $\operatorname{cat}(t, x)$ model is analogous.

\subsection{The second optimization problem}

We consider the second optimization problem

$$
\min _{\text {cat } \in \mathbf{A}} \mathcal{V}(c a t)=\min _{\text {cat } \in \mathbf{A}} \int_{T_{1}}^{T} \int_{0}^{1} r^{2}(c a t ; t, x) d x d t,
$$

subjects to Problem $I_{a}, I I_{a}$, and

$$
\min _{c a t \in \mathbf{A}_{c a t_{0}}} \mathcal{V}\left(c a t_{0}\right)=\min _{c a t_{0} \in \mathbf{A}_{c a t_{0}}} \int_{T_{1}}^{T} \int_{0}^{1} r^{2}\left(c a t_{0} ; t, x\right) d x d t .
$$

subjects to $I_{b}, I I_{b}$. First, we consider the second optimization problem subjects to Problem $I_{a}$ and $I I_{a}$. The one subjects to Problem $I_{b}, I I_{b}$ will be similarly briefly presented later.

Due to the continuous embedding $L^{2}\left(Q_{T}\right) \in \mathbf{X}_{T}$, the projection operator:

$$
\begin{aligned}
\mathbf{P}_{T_{1}, T}: \mathbf{X}_{T} & \longrightarrow L^{2}\left(\left[T_{1}, T\right] \times \Omega\right) \\
r(\text { cat } ; t, x) & \left.\longmapsto r(\text { cat } ; t, x)\right|_{\left[T_{1}, T\right]}
\end{aligned}
$$

is linear bounded. Denoting by $<.$, . $>$ the inner product in the Hilbert space $L^{2}\left(Q_{T}\right)$ and $<$., . $>_{L^{2}\left(\left[T_{1}, T\right] \times \Omega\right)}$ the inner product in the Hilbert space $L^{2}\left(\left[T_{1}, T\right] \times \Omega\right)$, we have the following Theorem: 
Theorem 4.8 Operator $\mathcal{V}$ in (1.37) is Frechet differentiable with respect to cat $\in W^{1, \infty}(\Omega)$.

Proof. Taking $\delta \in W^{1, \infty}(\Omega)$, we have

$$
\begin{array}{r}
\mathcal{V}(c a t+\delta)-\mathcal{V}(c a t)=\left\|\mathbf{P}_{T_{1}, T} r(c a t+\delta)\right\|_{L^{2}\left(\left[T_{1}, T\right] \times \Omega\right)}^{2}-\left\|\mathbf{P}_{T_{1}, T} r(c a t)\right\|_{L^{2}\left(\left[T_{1}, T\right] \times \Omega\right)}^{2}= \\
=\left\|\mathbf{P}_{T_{1}, T} r(c a t+\delta)-\mathbf{P}_{T_{1}, T} r(c a t)+\mathbf{P}_{T_{1}, T} r(c a t)\right\|_{L^{2}\left(\left[T_{1}, T\right] \times \Omega\right)}^{2}-\left\|\mathbf{P}_{T_{1}, T} r(c a t)\right\|_{L^{2}\left(\left[T_{1}, T\right] \times \Omega\right)}^{2}= \\
=2<\mathbf{P}_{T_{1}, T}\left(r(c a t+\delta)-r(c a t), \mathbf{P}_{T_{1}, T} r(c a t)>_{L^{2}\left(\left[T_{1}, T\right] \times \Omega\right)}+\left\|\mathbf{P}_{T_{1}, T}(r(c a t+\delta)-r(c a t))\right\|_{L^{2}\left(\left[T_{1}, T\right] \times \Omega\right)}^{2}\right.
\end{array}
$$

Similarly to the proof of Theorem 4.3, using equation (4.4) and Theorem about continuity of $(r, c)$ in $W^{1, \infty}\left(Q_{T}\right) \times \mathbf{Y}_{T}$ with respect to cat in $W^{1, \infty}(\Omega)$, we continue (4.17) as follows.

$$
\mathcal{V}(c a t+\delta)-\mathcal{V}(c a t)=2<\mathbf{P}_{T_{1}, T} r_{c a t} \delta, \mathbf{P}_{T_{1}, T} r(c a t)>_{L^{2}\left(\left[T_{1}, T\right] \times \Omega\right)}+o\left(\|\delta\|_{W^{1, \infty}(\Omega)}\right)
$$

Hence, we can define the linear bounded operator:

$$
\begin{aligned}
\mathcal{V}_{c a t}: W^{1, \infty}(\Omega) & \longrightarrow L\left(W^{1, \infty}(\Omega), \mathbb{R}\right) \\
c a t & \longmapsto \mathcal{V}_{c a t}(c a t)=2<\mathbf{P}_{T_{1}, T} r_{c a t} \cdot, \mathbf{P}_{T_{1}, T} r(c a t)>_{L^{2}\left(\left[T_{1}, T\right] \times \Omega\right)} .
\end{aligned}
$$

Remark 4.9 For convenience, we use the abbreviation $\mathcal{V}_{\text {cat }}$ for $\mathcal{V}_{\text {cat }}($.$) .$

We use the admissible set $\boldsymbol{A}$ defined in (4.10), we have the following result:

Theorem 4.10 The problem of minimizing $\mathcal{V}($ cat $)$ for cat $(x)$ model over $\boldsymbol{A}$ admits at least one solution.

For the simplicity to execute the numerical implementation (see Chapter 5), we will consider the original model (without Huber function adjustment) in a Hilbert space approach. We learn from the paper of Gerken and Lechleiter [15] and the book [35] to simplify the adjoint operators of derivative $r_{c a t}$ and $c_{c a t}$ by artificially changing into a Hilbert space framework. Suppose that there exist the linear bounded operator

$$
\begin{aligned}
r_{c a t}: L^{2}(\Omega) & \longrightarrow L^{2}\left(Q_{T}\right), \\
\delta & \longmapsto r_{c a t} \delta \\
c_{c a t}: L^{2}(\Omega) & \longrightarrow L^{2}\left(Q_{T}\right), \\
\delta & \longmapsto c_{c a t} \delta
\end{aligned}
$$

and linear bounded projection operator

$$
\begin{aligned}
\mathbf{P}_{T_{1}, T}: L^{2}\left(Q_{T}\right) & \longrightarrow L^{2}\left(\left[T_{1}, T\right] \times \Omega\right) \\
r(c a t ; t, x) & \left.\longmapsto r(c a t ; t, x)\right|_{\left[T_{1}, T\right]} .
\end{aligned}
$$


Then there exist the adjoint operator

$$
\begin{aligned}
r_{c a t}^{*}: L^{2}\left(Q_{T}\right) & \longrightarrow L^{2}(\Omega) \\
z & \longmapsto r_{c a t}^{*} z .
\end{aligned}
$$

We denote function $z$ as follows:

$$
z(c a t ; t, x)=\left\{\begin{array}{l}
0, \text { if } t \in\left[0, T_{1}\right), \\
r(c a t ; t, x), \text { if } t \in\left[T_{1}, T\right] .
\end{array}\right.
$$

Our objective functional is:

$$
\begin{aligned}
\mathcal{V}(\text { cat })=\int_{0}^{1} \int_{T_{1}}^{T}(r(\text { cat } ;, x))^{2} d x d t & =\int_{0}^{1} \int_{T_{1}}^{T}\left(\mathbf{P}_{T_{1}, T} r(\text { cat } ; t, x)\right)^{2} d x d t= \\
& =\int_{0}^{1} \int_{0}^{T} z^{2}(c a t ; t, x) d x d t .
\end{aligned}
$$

We have the following results.

Theorem 4.11 The Frechet derivative $\mathcal{V}_{\text {cat }}$ is defined by $2\left(r_{\text {cat }}\right)^{*}\left(\mathbf{P}_{T_{1}, T}\right)^{*} \mathbf{P}_{T_{1}, T} r($ cat $)$.

Remark 4.12 The Frechet derivative $\mathcal{V}_{\text {cat }}$ can also be calculated by $2 r_{\text {cat }}^{*} z(c a t)$, where $z($ cat $)$ is defined in (4.23).

Proof. As $r_{\text {cat }}$ is linear, using $z(c a t)=0$ on $\left[0, T_{1}\right)$, from (4.24) we have

$$
\begin{aligned}
& \mathcal{V}(c a t+\delta)-\mathcal{V}(c a t) \stackrel{(4.17)}{=} 2 \int_{0}^{1} \int_{T_{1}}^{T} r_{c a t} \delta r(c a t) d x d t+o\left(\|\delta\|_{W^{1, \infty}(\Omega)}\right)= \\
& =2<z_{c a t} \delta, z(c a t)>+o\left(\|\delta\|_{W^{1, \infty}(\Omega)}\right)=2<r_{c a t} \delta, z(c a t)>=2\left(\delta, r_{c a t}^{*} z(c a t)\right)+o\left(\|\delta\|_{W^{1, \infty}(\Omega)}\right) .
\end{aligned}
$$

Remark 4.13 From Theorem 4.11 and Remark 4.12, we see that

$$
\left(\mathbf{P}_{T_{1}, T}\right)^{*} \mathbf{P}_{T_{1}, T} r(c a t)=z(c a t ; t, x)=\left\{\begin{array}{l}
0, \text { if } t \in\left[0, T_{1}\right), \\
r(c a t ; t, x), \text { if } t \in\left[T_{1}, T\right] .
\end{array}\right.
$$

\subsubsection{Calculation of the adjoint operator $r_{c a t}^{*}$ for Problem $I_{a}$}

For simplicity we will not work with the reformulated model with Huber function in Chapter 3 in solving optimization problems. We recall original Problem $I_{a}$ :

$$
\frac{\partial}{\partial t}\left(r^{2} c\right)=\frac{\partial}{\partial x}\left(r^{2} \frac{\partial c}{\partial x}\right)-\beta(t, r, c) r c,(t, x) \in Q_{T}
$$




$$
\begin{array}{r}
\frac{\partial r}{\partial t}=-\gamma(t, r, c) \operatorname{cat}(x) c,(t, x) \in Q_{T}, \\
r(0, x)=r_{0}(x)=1, x \in \Omega, \\
c(0, x)=h(x), \\
c(t, 0)=1, t \in[0, T], \\
c_{x}(t, 1)=0,
\end{array}
$$

With assumption of smoothness on $r, c$ and positivity of $r$, the above system leads us to

$$
\begin{array}{r}
c_{t}=c_{x x}+\frac{2 r_{x} c_{x}}{r}-\frac{\left(2 r_{t}+\frac{D_{1}}{\int_{0}^{1} r c d x}\right) c}{r},(t, x) \in Q_{T}, \\
r_{t}=-\frac{D_{2}}{\int_{0}^{1} r c d x} \operatorname{cat}(x) c,(t, x) \in Q_{T}, \\
r(0, x)=r_{0}(x)=1, x \in \Omega, \\
c(0, x)=h(x), \\
c(t, 0)=1, t \in[0, T], \\
c_{x}(t, 1)=0 .
\end{array}
$$

where $\gamma$ and $\beta$ are defined in (1.18).

Now we introduce the following theorem about sensitivity system:

Theorem 4.14 (Sensitivity system). Suppose that there exists a solution of the problem for the original model in space $\mathbf{X}_{T} \times \mathbf{Y}_{T}$, suppose also that $\eta$ and $\varphi$ are denoted in (3.104). Then for $\delta \in W^{1, \infty}(\Omega)$, we get the sensibility system

$$
\eta_{t}+\frac{D_{2} c a t \varphi}{\int_{0}^{1} r c d x}-\frac{D_{2} c a t c}{\left(\int_{0}^{1} r c d x\right)^{2}} \int_{0}^{1} r \varphi+c \eta d x=-\frac{D_{2} \delta c}{\int_{0}^{1} r c d x}
$$

with the initial condition

$$
\begin{gathered}
\eta(0, x)=0 \\
\varphi_{t}-\varphi_{x x}-\frac{2 r_{x} \varphi_{x}}{r}+\left(\frac{2 r_{t}}{r}+\frac{D_{1}}{r \int_{0}^{1} r c d x}\right) \varphi=\frac{2 c_{x} \eta_{x}}{r}-\frac{2 c \eta_{t}}{r} \\
+\left(\frac{2 r_{t} c}{r^{2}}-\frac{2 r_{x} c_{x}}{r^{2}}+\frac{D_{1} c}{r^{2} \int_{0}^{1} r c d x}\right) \eta+\frac{D_{1} c}{r\left(\int_{0}^{1} r H_{\epsilon}(c) d x\right)^{2}} \int_{0}^{1} c \eta+r c \varphi d x,
\end{gathered}
$$

with initial and boundary conditions 


$$
\begin{aligned}
& \varphi(0, x)=0, \\
& \varphi(t, 0)=0, \\
& \varphi_{x}(t, 1)=0 .
\end{aligned}
$$

Proof. We use the same technique like in Section 3.4.1.

We will apply an adjoint technique by introducing an adjoint system corresponding to the sensitivity system in order to calculate $r_{c a t}^{*} z$ (cat).The idea of defining $r_{c a t}^{*} z(c a t)$ can be briefly presented in the following Remark.

Remark 4.15 From the Theorem 4.14, we have the sensitivity system:

$$
\begin{gathered}
D_{1} \varphi+L_{1} \eta=l \delta \\
D_{2} \varphi+L_{2} \eta=0,
\end{gathered}
$$

where $l=-\frac{D_{2} c}{\int_{0}^{1} r c d x}$ and $D_{j}, L_{j}, j=1,2$ are the linear differential and integral operators.

Testing system (4.32) by $v(t), w(t)$, with the conditions $v(T)=w(T)=0$ leads us to:

$$
\begin{array}{r}
<\widetilde{D}_{1} v, \varphi>+<\widetilde{L}_{1} v, \eta>=<l \delta, v>, \\
<\widetilde{D}_{2} w, \varphi>+<\widetilde{L}_{2} w, \eta>=0,
\end{array}
$$

Summing up the equation from the system (4.33), we deduce:

$$
<\varphi, \widetilde{D}_{1} v+\widetilde{D}_{2} w>+<\eta, \widetilde{L}_{1} v+\widetilde{L}_{2} w>=<\delta, l v>.
$$

In order to determine $r_{\text {cat }}^{*} z(c a t)$, we solve the system

$$
\begin{aligned}
& \widetilde{D}_{1} v+\widetilde{D}_{2} w=0 \\
& \widetilde{L}_{1} v+\widetilde{L}_{2} w=z,
\end{aligned}
$$

then it leads to

$$
\begin{gathered}
<\eta, z>=<\delta, l v>, \\
\Leftrightarrow\left(\delta, r_{c a t}^{*} z\right)=<\delta, l v>,
\end{gathered}
$$

Hence we have

$$
r_{c a t}^{*} z(c a t)=\int_{0}^{T} l v d t
$$

The next Theorem will illustrate the idea mentioned in the above Remark. 
Theorem 4.16 (Adjoint system.) Suppose that the following system

$$
\begin{array}{r}
-w_{t}-w_{x x}+\frac{2 r_{x}}{r} w_{x}+\left(\frac{2 r_{x x} r-2 r_{x}^{2}+2 r_{t} r}{r^{2}}+\frac{D_{1}}{r \int_{0}^{1} r c d x}\right) w+\frac{D_{2} c a t}{\int_{0}^{1} r c d x} v-r \int_{0}^{1} \frac{D_{2} c a t c}{\left(\int_{0}^{1} r c d x\right)^{2}} v d x- \\
-r \int_{0}^{1} \frac{D_{1} c w}{r\left(\int_{0}^{1} r c d x\right)^{2}} d x=0,
\end{array}
$$

$$
\begin{aligned}
& -\frac{2 c}{r} w_{t}+\frac{2 c_{x}}{r} w_{x}-\left(\frac{D_{1} c}{r^{2} \int_{0}^{1} r c d x}-\frac{2 c_{x x}}{r}+\frac{2 c_{t} r}{r^{2}}\right) w-c \int_{0}^{1} \frac{D_{1} c w}{r\left(\int_{0}^{1} r c d x\right)^{2}} d x- \\
& -v_{t}-c \int_{0}^{1} \frac{D_{2} c a t c}{\left(\int_{0}^{1} r c d x\right)^{2}} v d x=z,
\end{aligned}
$$

and the conditions

$$
\begin{aligned}
& v(T, x)=0, \\
& w(T, x)=0, \\
& w(t, 0)=0, \\
& w_{x}(t, 1)=0
\end{aligned}
$$

has unique solution $(v, w) \in W^{1, \infty}\left(Q_{T}\right) \times W(0, T)$ with some positive $T$, and $z$ defined in (4.23).

Suppose additionally a boundary condition on cat $(x)$

$$
\operatorname{cat}_{x}(1)=0 .
$$

Then we have

$$
V_{c a t}=\int_{0}^{T} \frac{D_{2} c}{\int_{0}^{1} r c d x} v d t
$$

Proof.

Denoting the coefficients from (4.28) by

$$
\begin{aligned}
& a_{1}(t, x)=\frac{D_{2} c a t}{\int_{0}^{1} r c d x}, \\
& a_{2}(t, x)=-\frac{D_{2} c a t c}{\left(\int_{0}^{1} r c d x\right)^{2}}, \\
& a_{3}(t, x)=-\frac{D_{2} c}{\int_{0}^{1} r c d x},
\end{aligned}
$$


and the coefficients from (4.30) by

$$
\begin{aligned}
& b_{1}(t, x)=-\frac{2 r_{x}}{r}, \\
& b_{2}(t, x)=\frac{2 r_{t}}{r}+\frac{D_{1}}{r \int_{0}^{1} r c d x}, \\
& b_{3}(t, x)=\frac{2 c_{x}}{r}, \\
& b_{4}(t, x)=-\frac{2 c}{r}, \\
& b_{5}(t, x)=\frac{2 r_{t} c}{r^{2}}-\frac{2 r_{x} c_{x}}{r^{2}}+\frac{D_{1} c}{r^{2} \int_{0}^{1} r c d x} \\
& b_{6}(t, x)=\frac{D_{1} c}{r\left(\int_{0}^{1} r c d x\right)^{2}} .
\end{aligned}
$$

Then (4.28) and (4.30) become

$$
\begin{gathered}
\eta_{t}+a_{1} \varphi+a_{2} \int_{0}^{1} r \varphi+a_{2} \int_{0}^{1} c \eta=a_{3} \delta, \\
\varphi_{t}-\varphi_{x x}+b_{1} \varphi_{x}+b_{2} \varphi=b_{3} \eta_{x}+b_{4} \eta_{t}+b_{5} \eta+b_{6} \int_{0}^{1} r \varphi d x+b_{6} \int_{0}^{1} c \eta d x .
\end{gathered}
$$

Testing (4.45) by $v \in W^{1, \infty}\left(Q_{T}\right)$, with the condition (4.39), we deduce

$$
<\varphi, a_{1} v+r \int_{0}^{1} a_{2} v d x>+<\eta,-v_{t}+c \int_{0}^{1} a_{2} v d x>=<\delta, a_{3} v>
$$

or

$$
<\varphi, \frac{D_{2} c a t}{\int_{0}^{1} r c d x} v-r \int_{0}^{1} \frac{D_{2} c a t c}{\left(\int_{0}^{1} r c\right)^{2}} v d x>+<\eta,-v_{t}-c \int_{0}^{1} \frac{D_{2} c a t c}{\left(\int_{0}^{1} r c\right)^{2}} v d x>=-<\delta, \frac{D_{2} c}{\int_{0}^{1} r c d x} v>,
$$

Testing (4.46) by the function $w \in W(0, T)$, where $w$ satisfies (4.40), we have

$$
\begin{aligned}
& <\varphi_{t}, w>-<\varphi_{x x}, w>+<b_{1} \varphi_{x}, w>+<b_{2} \varphi, w>= \\
& =<b_{3} \eta_{x}, w>+<b_{4} \eta_{t}, w>+<b_{5} \eta, w>+<b_{6} \int_{0}^{1} r \varphi d x, w>+<b_{6} \int_{0}^{1} c \eta d x, w>.
\end{aligned}
$$


Due to assumption (4.73) and (4.27b), we see that

$$
r_{x}(1)=0,
$$

which leads to $b_{1}(t, 1)=0$ and $b_{3}(t, 1)=0$. We therefore we obtain

$$
\begin{aligned}
& <\varphi,-w_{t}-w_{x x}+\frac{2 r_{x}}{r} w_{x}+\left(\frac{2 r_{x x} r-2 r_{x}^{2}+2 r_{t} r}{r^{2}}+\frac{D_{1}}{r \int_{0}^{1} r c d x}\right) w-r \int_{0}^{1} \frac{D_{1} c}{r\left(\int_{0}^{1} r c d x\right)^{2}} w d x>+ \\
& +<\eta,-\frac{2 c}{r} w_{t}+\frac{2 c_{x}}{r} w_{x}-\left(\frac{D_{1} c}{r^{2} \int_{0}^{1} r c d x}-\frac{2 c_{x x}}{r}+\frac{2 c_{t} r}{r^{2}}\right) w-c \int_{0}^{1} \frac{D_{1} c w}{r\left(\int_{0}^{1} r c d x\right)^{2}} d x>=0 .
\end{aligned}
$$

Summing up (4.48) and (4.51), we get

$$
\begin{aligned}
& <\varphi,-w_{t}-w_{x x}+\frac{2 r_{x}}{r} w_{x}+\left(\frac{2 r_{x x} r-2 r_{x}^{2}+2 r_{t} r}{r^{2}}+\frac{D_{1}}{r \int_{0}^{1} r c d x}\right) w- \\
& -r \int_{0}^{1} \frac{D_{1} c}{r\left(\int_{0}^{1} r c d x\right)^{2}} w d x+\frac{D_{2} c a t}{\int_{0}^{1} r c d x} v-r \int_{0}^{1} \frac{D_{2} c a t c}{\left(\int_{0}^{1} r c d x\right)^{2}} v d x>+ \\
& +<\eta,-\frac{2 c}{r} w_{t}+\frac{2 c_{x}}{r} w_{x}-\left(\frac{D_{1} c}{r^{2} \int_{0}^{1} r c d x}-\frac{2 c_{x x}}{r}+\frac{2 c_{t} r}{r^{2}}\right) w-c \int_{0}^{1} \frac{D_{1} c w}{r\left(\int_{0}^{1} r c d x\right)^{2}} d x- \\
& -v_{t}-c \int_{0}^{1} \frac{D_{2} c a t c}{\left(\int_{0}^{1} r c d x\right)^{2}} v d x>=-<\delta, \frac{D_{2} c}{\int_{0}^{1} r c d x} v>.
\end{aligned}
$$

From the hypothesis of this Theorem that the system (4.67) - (4.40) is solvable. Then

Hence

$$
<\eta, z>=-<\delta, \frac{D_{2} c}{\int_{0}^{1} r c d x} v>\text {. }
$$

$$
\left(\delta, r_{c a t}^{*} z\right)=-<\delta, \frac{D_{2} c}{\int_{0}^{1} r c d x} v>
$$

We can rewrite (4.53) as

$$
\begin{aligned}
& \int_{0}^{1} \delta r_{c a t}^{*} z d x=-\int_{0}^{1} \int_{0}^{T} \delta \frac{D_{2} c}{\int_{0}^{1} r c d x} v d x d t \\
\Leftrightarrow & \int_{0}^{1} \delta\left(r_{c a t}^{*} z-\int_{0}^{T} \frac{D_{2} c}{\int_{0}^{1} r c d x} v d t\right) d x=0 .
\end{aligned}
$$


As (4.53) is satisfied $\forall \delta \in L^{2}(\Omega)$, we get

$$
r_{c a t}^{*} z=-\int_{0}^{T} \frac{D_{2} c}{\int_{0}^{1} r c d x} v d t
$$

Thus we arrive at (4.42).

\subsubsection{Calculation of the adjoint operator $r_{c a t}^{*}$ for Problem $I_{b}$}

The Problem $I_{b}$ leads to system

$$
\begin{array}{r}
c_{t}=c_{x x}+\frac{2 r_{x} c_{x}}{r}-\frac{\left(2 r_{t}+\frac{D_{1}}{\int_{0}^{1} r c d x}\right) c}{r},(t, x) \in Q_{T}, \\
r_{t}=-\frac{D_{2}}{\int_{0}^{1} r c d x} \operatorname{cat}(t, x) c,(t, x) \in Q_{T}, \\
r(0, x)=r_{0}(x)=1, x \in \Omega, \\
c(0, x)=h(x), \\
c(t, 0)=1, t \in[0, T], \\
c_{x}(t, 1)=0,
\end{array}
$$

where cat $(\mathrm{t}, \mathrm{x})$ is defined by $(1.25)$

$$
\left.\operatorname{cat}_{t}(t, x)=\alpha_{0} \int_{0}^{1} H_{R_{0}}(r(c a t ; y, t)) G(x-y)\right) d y,
$$

and the initial condition

$$
\operatorname{cat}(0, x)=\operatorname{cat}_{0}(x)
$$

with given $\alpha_{0}, R_{0}$. Functions $H$ and $G$ are defined in Appendix A.2 and A.8.

From Chapter 3, we know that $r \in \mathbf{X}_{T}, c \in \mathbf{Y}_{T}$, cat $\in W^{1, \infty}(\Omega)$ are Fréchet differentiable with respect to $c a t_{0} \in W^{1, \infty}(\Omega)$ for some $T$ small enough, we denote

$$
\bar{\eta}:=r_{c a t_{0}} \Delta, \bar{\varphi}:=c_{c a t_{0}} \Delta, \psi:=\operatorname{cat}_{c a t_{0}} \Delta .
$$

We have the following results.

Theorem 4.17 Suppose that there exists a solution of the original model of Problem $I_{b}$ in space $\mathbf{X}_{T} \times \mathbf{Y}_{T}$. Then we get the sensitivity system of three equations as follows:

$$
\bar{\eta}_{t}=-\frac{D_{2}}{\int_{0}^{1} r c d x}\left(c \psi+c a t \bar{\varphi}-\frac{c a t c}{\int_{0}^{1} r c d x} \int_{0}^{1} r \bar{\varphi}+c \bar{\eta} d x\right),
$$


with the initial condition

$$
\begin{gathered}
\bar{\eta}(0, x)=0 \\
\bar{\varphi}_{t}-\bar{\varphi}_{x x}-\frac{2 r_{x} \bar{\varphi}_{x}}{r}+\left(\frac{2 r_{t}}{r}+\frac{D_{1}}{r \int_{0}^{1} r c d x}\right) \bar{\varphi}=\frac{2 c_{x} \bar{\eta}_{x}}{r}-\frac{2 c \bar{\eta}_{t}}{r} \\
+\left(\frac{2 r_{t} c}{r^{2}}-\frac{2 r_{x} c_{x}}{r^{2}}+\frac{D_{1} c}{r^{2} \int_{0}^{1} r c d x}\right) \bar{\eta}+\frac{D_{1} c}{r\left(\int_{0}^{1} r H_{\epsilon}(c) d x\right)^{2}} \int_{0}^{1} c \bar{\eta}+r c \bar{\varphi} d x,
\end{gathered}
$$

with initial and boudary conditions

$$
\begin{aligned}
& \bar{\varphi}(0, x)=0, \\
& \bar{\varphi}(t, 0)=0, \\
& \bar{\varphi}_{x}(t, 1)=0,
\end{aligned}
$$

and

$$
\psi_{t}=\alpha_{0} \int_{0}^{1} G(x-y) H_{R_{0}}^{\prime}\left(r\left(c a t_{0} ; y, t\right)\right) \bar{\eta}(y, t) d y,
$$

with initial condition

$$
\psi(0, x)=\Delta(x) .
$$

Proof. The proof of this Theorem follows the same technique in the Section 3.4.1

We denote also function $\mathbf{z}$

$$
\mathbf{z}\left(\text { cat }_{0} ; t, x\right)=\left\{\begin{array}{l}
0, \text { if } t \in\left[0, T_{1}\right) \\
\overline{\mathbf{P}}_{T_{1}, T} r\left(c_{0} ; t, x\right), \text { if } t \in\left[T_{1}, T\right]
\end{array}=\left\{\begin{array}{l}
0, \text { if } t \in\left[0, T_{1}\right) \\
r\left(\text { cat }_{0} ; t, x\right), \text { if } t \in\left[T_{1}, T\right]
\end{array}\right.\right.
$$

Our objective functional is:

$$
\left.V\left(c a t_{0}\right):=V\left(c a t_{0} ; t, x\right)\right)=\int_{0}^{1} \int_{T_{1}}^{T}\left(r\left(c a t_{0} ; t, x\right)\right)^{2} d x d t=\int_{0}^{1} \int_{0}^{T}\left(\mathbf{z}\left(c a t_{0} ; t, x\right)\right)^{2} d x d t .
$$

Theorem 4.18 Operator $\mathcal{V}$ in (1.38) is Frechet differentiable with respect to cat $t_{0}$, and the Frechet derivative is defined by $2\left(r_{\text {cat }}\right)^{*}\left(\mathbf{P}_{T_{1}, T}\right)^{*} \mathbf{P}_{T_{1}, T} r\left(\right.$ cat $\left._{0}\right)$. 
Proof. The proof of this Theorem is similar to the one of Theorem 4.11.

Remark 4.19 The Frechet derivative $\mathcal{V}_{\text {cat }}$ can also be calculated by $2 r_{\text {cat }}^{*} \mathbf{z}($ cat $)$, where $\mathbf{z}\left(\right.$ cat $\left._{0}\right)$ is defined in (4.64).

Proof. Using $\mathbf{z}\left(\right.$ cat $\left._{0}\right)=0$ on $\left[0, T_{1}\right)$ and $r_{\text {cat }}$ are linear

$$
\begin{aligned}
& V\left(c_{a t}+\Delta\right)-V\left(c_{0}\right)=2 \int_{0}^{1} \int_{T_{1}}^{T_{2}} r_{c a t_{0}} \Delta r\left(c a t_{0}\right) d x d t+o\left(\|\Delta\|_{W^{1, \infty}(\Omega)}\right)= \\
& =2<\mathbf{z}_{c a t_{0}} \Delta, \mathbf{z}\left(c a t_{0}\right)>+o\left(\|\Delta\|_{W^{1, \infty}(\Omega)}\right)=2<r_{c a t_{0}} \Delta, \mathbf{z}\left(\operatorname{cat}_{0}\right)>=2<\Delta, r_{c a t_{0}}^{*} \mathbf{z}\left(\operatorname{cat}_{0}\right)>+o\left(\|\Delta\|_{W^{1, \infty}(\Omega)}\right) .
\end{aligned}
$$

Remark 4.20 From Theorem 4.18 and Remark 4.19, we see that

$$
\left(\mathbf{P}_{T_{1}, T}\right)^{*} \mathbf{P}_{T_{1}, T} r\left(c a t_{0}\right)=\mathbf{z}\left(\text { cat }_{0} ; t, x\right)=\left\{\begin{array}{l}
0, \text { if } t \in\left[0, T_{1}\right), \\
r\left(c a t_{0} ; t, x\right), \text { if } t \in\left[T_{1}, T\right] .
\end{array}\right.
$$

In the folowing Theorem, we will introduce adjoint system to sensitivity in order to calculate $r_{\text {cat }}^{*} \mathbf{z}\left(\right.$ cat $\left._{0}\right)$. The brief idea for this Theorem is similar the one in the Remark 4.15.

Theorem 4.21 Suppose that the following system

$$
\begin{gathered}
-w_{t}-w_{x x}+\frac{2 r_{x}}{r} w_{x}+\left(\frac{2 r_{x x} r-2 r_{x}^{2}+2 r_{t} r}{r^{2}}+\frac{D_{1}}{r \int_{0}^{1} r c d x}\right) w+\frac{D_{2} c a t}{\int_{0}^{1} r c d x} v- \\
-r \int_{0}^{1} \frac{D_{2} c a t c}{\left(\int_{0}^{1} r c d x\right)^{2}} v d x-r \int_{0}^{1} \frac{D_{1} c w}{r\left(\int_{0}^{1} r c d x\right)^{2}} d x=0, \\
-\frac{2 c}{r} w_{t}+\frac{2 c_{x}}{r} w_{x}-\left(\frac{D_{1} c}{r^{2} \int_{0}^{1} r c d x}-\frac{2 c_{x x}}{r}+\frac{2 c_{t} r}{r^{2}}\right) w-c \int_{0}^{1} \frac{D_{1} c w}{r\left(\int_{0}^{1} r c d x\right)^{2}} d x- \\
-v_{t}-c \int_{0}^{1} \frac{D_{2} c a t c}{\left(\int_{0}^{1} r c d x\right)^{2}} v d x+\alpha_{0} H_{R_{0}}^{\prime}\left(c a t_{0}, x, t\right) \int_{0}^{1} \zeta(y, t) G(y-x) d y=\mathbf{z},
\end{gathered}
$$

with the conditions

$$
\begin{aligned}
& v(T, x)=0, \\
& w(T, x)=0 \\
& w(t, 0)=0 \\
& w_{x}(t, 1)=0,
\end{aligned}
$$


and equation

$$
\zeta_{t}=-\frac{D_{2} c v}{\int_{0}^{1} r c d x}
$$

with condition

$$
\zeta(T, x)=0 .
$$

has unique solution $(v, w$, cat $) \in W^{1, \infty}\left(Q_{T}\right) \times W(0, T) \times W^{1, \infty}(\Omega)$.

Suppose additionally a boundary condition on cat $(x)$

$$
\operatorname{cat}_{0 x}(1)=0 .
$$

Then

$$
V_{c a t_{0}}=-\zeta(0, x)
$$





\section{Chapter 5}

\section{Numerical Implementation}

\subsection{Forward Problems}

In this section we will consider the numerical implementation for forward problems I and II and the two optimization problems for Problem $I_{a}$. The optimization problem for Problem $I_{b}$ shows the similar results as the ones for $I_{a}$. First, we introduce the Finite Element Method (FEM) to solve the forward problems and give a short investigation on the greedy algorithm that is presented in [8], where the authors approach to Problems $I I$ and $I I_{b}$ by the Finite Difference Method. We will also make some comparisons between the Finite Difference Method and the Finite Element Method for problem $I I$. Finally, the two optimization problems are considered by the sensitivity technique and fast derivative calculation by solving the adjoint systems.

In Problem $I$, we will find $c(t, x)$ and $r(t, x)$ such that

$$
\begin{gathered}
\frac{\partial}{\partial t}\left(r^{2} c\right)=\frac{\partial}{\partial x}\left(r^{2} \frac{\partial c}{\partial x}\right)-\beta(t, r, c) r^{2} c,(t, x) \in Q_{T}, \\
\frac{\partial r}{\partial t}=-\gamma(t, r, c) c,(t, x) \in Q_{T}, \\
r(0, x)=r_{0}(x)=1, x \in \Omega, \\
c(0, x)=h(x), \\
c(t, 0)=1, t \in[0, T], \\
c_{x}(t, 1)=0, t \in[0, T],
\end{gathered}
$$


In the problem $I I$, the equation (5.1) is replaced by

$$
\frac{\partial}{\partial t}\left(r^{\frac{4}{3}} c\right)=\frac{\partial}{\partial x}\left(r^{2} \frac{\partial c}{\partial x}\right)-\beta(t, r, c) r^{\frac{1}{3}} c,(t, x) \in Q_{T},
$$

Suppose that $r>r_{0}$, where $r_{0}$ is some positive number, we rewrite the equations (5.1), (5.4), (5.5) in the form of parabolic problem:

$$
c_{t}-c_{x x}-\frac{2 r_{x} c_{x}}{r}+\frac{\left(2 r_{t}+\beta\right) c}{r}=0,(t, x) \in Q_{T},
$$

and the equations (5.7), (5.4), (5.5) in the form

$$
c_{t}-r^{\frac{2}{3}} c_{x x}-\frac{2 r_{x} c_{x}}{r^{\frac{1}{3}}}+\frac{\left(\frac{4}{3} r_{t}+\beta\right) c}{r}=0,(t, x) \in Q_{T} .
$$

The authors of [8] solved numerically the above system by dealing with every time step $\Delta t$ starting with the Cauchy equation (5.2) and then the equation (5.9). They applied FDM to Problem $I I$ and $I I_{b}$. The main idea of the FDM is the approximation of partial derivatives with the difference quotients of values of functions pore radius $r$ and oxygen concentration $c$. They did experiments with finite explicit method in time and both implicit and explicit methods in space. Finally, they concluded that the greedy algorithm works faster with the implicit one.

$N, J$ are some natural numbers, the equidistant grids are

$$
\begin{gathered}
x_{j}=j \Delta x, j=0, \ldots, J-1 \\
t_{n}=n \Delta t, n=0,1, \cdots
\end{gathered}
$$

where $\Delta x=\frac{1}{J-1}$ and $\Delta t=\frac{1}{N-1}$ are the spacial and time steps alternatively.

The partial differential equation (5.2) determining the decrease of radius $r$ is discretized and the radius in the next time step is calculated via

$$
r_{j}^{n+1}=r_{j}^{n}-\Delta t \gamma c_{j}^{n}, \text { for } j=0, . ., J-1, n=0,1, \cdots
$$

with the discrete initial conditions for $n=0$

$$
r_{j}^{0}=1 \text { for } j=0, \ldots, J-1,
$$

the Dirichlet boundary condition for $j=0$

$$
c_{0}^{n}=1 \text { for } n=0,1, \cdots
$$

and the Neumann condition

$$
c_{J-2}^{n}=c_{J-1}^{n} \text { for } n=0,1, \cdots
$$

In those notions the derivatives are written as

$$
\begin{array}{r}
r_{x} \approx \frac{r_{j}^{n}-r_{j-1}^{n}}{\Delta x}, \\
c_{x} \approx \frac{c_{j}^{n+1}-c_{j-1}^{n+1}}{\Delta x}, \\
c_{x x} \approx \frac{c_{j-1}^{n+1}-2 c_{j}^{n+1}+c_{j+1}^{n+1}}{(\Delta x)^{2}},
\end{array}
$$


Then it leads to solving the following system of $(J-1)$ algebraic equations

$$
A c^{n+1}=c^{n}-\operatorname{corr}^{n}
$$

where $\operatorname{corr}^{n}=\left(a_{10}^{n} \Delta c_{0}^{n+1}, 0, \ldots, 0\right)^{T}$ is a correction vector due to the mixed boundary condition. The $(J-1) \times(J-1)$ matrix $A$ is given by:

$$
A=\left[\begin{array}{ccccc}
a_{11}^{n} & a_{12}^{n} & 0 & \cdots & 0 \\
a_{21}^{n} & a_{22}^{n} & a_{23}^{n} & \cdots & 0 \\
\cdots & \cdots & \cdots & \cdots & \cdots \\
0 & \cdots & a_{J-2 J-3}^{n} & a_{J-2 J-2}^{n} & a_{J-2 J-1}^{n} \\
0 & \cdots & \cdots & a_{J-1 J-2}^{n} & a_{J-1 J-1}^{n}+a_{J-1 J}^{n}
\end{array}\right]
$$

with elements

$$
\begin{array}{r}
a_{j j-1}^{n}=\frac{\Delta t}{\Delta x^{2}}\left(r_{j}^{n}\right)^{\frac{2}{3}}, \\
a_{j j}^{n}=\frac{\Delta t}{\Delta x^{2}}\left(r_{j}^{n}\right)^{-\frac{1}{3}} 2\left(r_{j-1}^{n}-2 r_{j}^{n}\right)+\left(r_{j}^{n}\right)^{-1}\left[\frac{1}{3}\left(7 r_{j}^{n}-4 r_{j}^{n+1}\right)-\beta \Delta t\right], \\
a_{j j+1}^{n}=\frac{\Delta t}{\Delta x^{2}}\left(r_{j}^{n}\right)^{-\frac{1}{3}}\left(3 r_{j}^{n}-2 r_{j-1}^{n}\right) .
\end{array}
$$

The calculation of $\beta$ and $\gamma$ leads to the knowledge of the reaction rate $k_{s}$. Dabrowski et al. approximated the integral in $k_{s}$ via the trapezoidal rule as the following:

$$
Q^{n}\left(r^{n}, c^{n}\right)=\Delta x\left[\frac{1}{2}\left(\left(r_{0}^{n}\right)^{\frac{1}{3}} c_{0}^{n}+\left(r_{J-1}^{n}\right)^{\frac{1}{3}} c_{J-1}^{n}\right)+\sum_{j=1}^{J-2}\left(r_{j}^{n}\right)^{\frac{1}{3}} c_{j}^{n} r_{j}^{n}\right],
$$

then we got the discrete reaction rate

$$
k_{s}^{n}=\frac{i r_{p}^{0}}{4 F \epsilon_{0} c_{A, 0} l_{c}} \frac{1}{Q^{n}\left(r^{n}, c^{n}\right)} .
$$

The stability condition for FDM is given by:

$$
\frac{\Delta t}{\Delta x} \geq \text { const. }\left[\frac{1}{2}\left(\left(r_{0}^{n}\right)^{\frac{1}{3}} c_{0}^{n}+\left(r_{J-1}^{n}\right)^{\frac{1}{3}} c_{J-1}^{n}\right)+\sum_{j=1}^{J-2}\left(r_{j}^{n}\right)^{\frac{1}{3}} c_{j}^{n} r_{j}^{n}\right]\left[\frac{1}{3}\left(7 r_{j}^{n}-4 r_{j}^{n+1}\right)-\left(r_{j}^{n}\right)^{-\frac{5}{3}}\right],
$$

and

$$
\frac{\Delta t}{\Delta x} \leq \text { const. }\left[\frac{1}{2}\left(\left(r_{0}^{n}\right)^{\frac{1}{3}} c_{0}^{n}+\left(r_{J-1}^{n}\right)^{\frac{1}{3}} c_{J-1}^{n}\right)+\sum_{j=1}^{J-2}\left(r_{j}^{n}\right)^{\frac{1}{3}} c_{j}^{n} r_{j}^{n}\right]\left[\frac{1}{3}\left(7 r_{j}^{n}-4 r_{j}^{n+1}\right)\right] .
$$

However, we see that, the condition (5.16) and (5.17) are not explicit, as the two sides of them depends on $\Delta t$ and $\Delta x$. 
Now we will develop the Finite Element method (FEM) for numerically solving the system of partial differential equations in the problems $I$ and $I I$. First denoting $u(t . x)=c(t, x)-1$, we obtain for the problem $I$ :

$$
\begin{gathered}
u_{t}-u_{x x}-\frac{2 r_{x} u_{x}}{r}+\frac{\left(2 r_{t}+\beta\right) u}{r}=-\frac{2 r_{t}+\beta}{r},(t, x) \in Q_{T}, \\
u(0, x)=h(x)-1, \\
u(t, 0)=0, t \in[0, T], \\
u_{x}(t, 1)=0, t \in[0, T],
\end{gathered}
$$

and the equation (5.18) is replaced by

$$
u_{t}-r^{\frac{2}{3}} u_{x x}-\frac{2 r_{x} u_{x}}{r^{\frac{1}{3}}}+\frac{\left(\frac{4}{3} r_{t}+\beta\right) u}{r}=-\frac{\frac{4}{3} r_{t}+\beta}{r},(t, x) \in Q_{T}
$$

for the problem $I I$.

Note that $\beta=\frac{D_{1}}{\int_{0}^{1} r c d c}, \gamma=\frac{D_{2}}{\int_{0}^{1} r c d c}$ in Problem $I$ and $\beta=\frac{D_{1}}{\int_{0}^{1} r^{\frac{1}{3}} c d c}, \gamma=\frac{D_{2}}{\int_{0}^{1} r^{\frac{1}{3}} c d c}$ in Problem II. Define the space

$$
V=\left\{v \in H^{1}(\Omega), v(0)=0, v_{x}(1)=0\right\}
$$

We will employ the backward Euler scheme for the time derivative term and the discretisation in the $x$ variable that is based on the finite element method. The stability of backward Euler scheme method was proved in many text books on FEM or numerical methods on soving PDEs, e.g. [33]. For solving system of PDEs in one dimensional spacial frame, we refer [34].

We define the piecewise basic functions as follows.

\subsubsection{Piecewise basic functions of the spaces $V_{h}$}

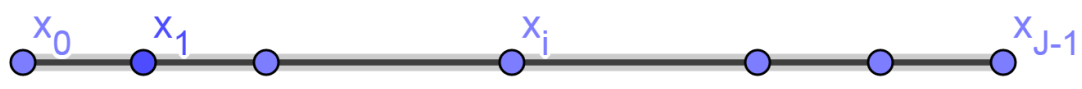

Figure 5.1: The subdivision of $\Omega=[0,1], x_{0}=0, x_{J-1}=1$

In order to solve our problem numerically we subdivide $\Omega=[0,1]$ into $J-1$ subintervals $\left[x_{i}, x_{i+1}\right], i=$ $0, \ldots, J-2$ by the points $x_{i}=i h$, where $i=0, \ldots, J-1$ and $h=1 /(J-1)$.The subdivision is expressed 


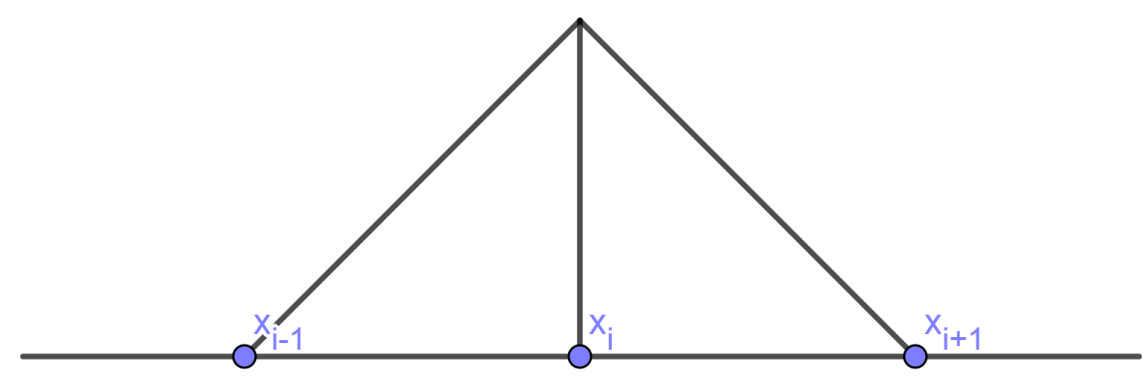

Figure 5.2: The piecewise linear finite element basis function $\Phi_{i}(x), i \in[1, J-3]$

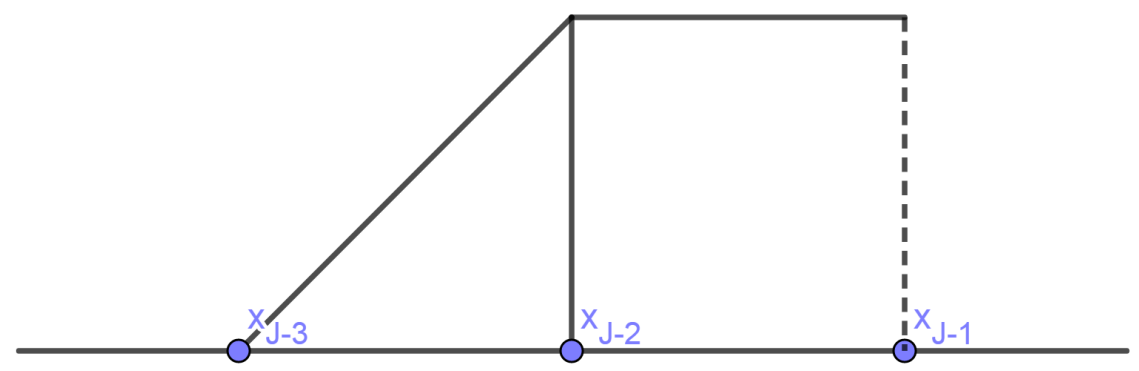

Figure 5.3: The piecewise linear finite element basis function $\Phi_{J-2}(x)$

in the Figure 5.1.

The weak solution $v \in V$ of the problem (5.19)-(5.53) will be approximated by a linear combination of the finite element basic piecewise linear functions with $x$-mesh point $x_{i}$ on the subdivisions $\Phi_{i}(x)$, depicted in the figures 5.2 and 5.3.

$$
\Phi_{i}(x)=\left(1-\left|\frac{x-x_{i}}{h}\right|\right)_{+}, \quad i=1, \ldots, J-3
$$

and

$$
\Phi_{J-2}(x)=\left\{\begin{array}{l}
1-\frac{x-x_{J-2}}{h}, \text { if } x \in\left[x_{J-3}, x_{J-2}\right] \\
1, \text { if } x \in\left[x_{J-2}, x_{J-1}\right]
\end{array}\right.
$$

It is clear that $\Phi_{i}(x) \in V, i=1, \ldots, J-2$; furthermore $\operatorname{supp} \Phi_{i}=\left[x_{i}, x_{i+1}\right], i=1, \ldots, J-2$.

The space

$$
V_{h}=\operatorname{span}\left(\Phi_{1}, \ldots, \Phi_{J-2}\right)
$$

is an $(J-2)$ dimensional subspace of $V$. 
We have the followings

$$
\begin{gathered}
\left(\Phi_{i}, \Phi_{j}\right)=\left\{\begin{array}{l}
\frac{2 h}{3}, \text { if } i=j<J-2, \\
\frac{4 h}{3}, \text { if } i=j=J-2, \\
\frac{h}{6}, \text { if }|i-j|=1, \\
0, \text { if }|i-j|>1,
\end{array}\right. \\
\left(\left(\Phi_{i}\right)_{x}, \Phi_{j}\right)=\left\{\begin{array}{l}
0, \text { if } i=j<J-2, \\
\frac{1}{2}, \text { if } i=j=J-2, \\
-\frac{1}{2}, \text { if }|i-j|=1, i<j, \\
\frac{1}{2}, \text { if }|i-j|=1, i>j, \\
0, \text { if }|i-j|>1,
\end{array}\right. \\
\left(\left(\Phi_{i}\right)_{x},\left(\Phi_{j}\right)_{x}\right)=\left\{\begin{array}{l}
\frac{2}{h}, \text { if } i=j<J-2, \\
\frac{1}{h}, \text { if } i=j=J-2, \\
-\frac{1}{h}, \text { if }|i-j|=1, \\
0, \text { if }|i-j|>1 .
\end{array}\right.
\end{gathered}
$$

\subsubsection{The $\theta$ scheme for numerical solving the problems $I$ and $I I$}

The finite element approximation of the two problems $I$ and $I I$ via $\theta$ scheme is as follows.

Find $u_{h}^{m} \in V_{h}, 0 \leq m \leq N-1$, such that

$$
\begin{aligned}
& \left(\frac{u_{h}^{m+1}-u_{h}^{m}}{\delta t}, \vartheta_{h}\right)+a\left(u_{h}^{m+\theta}, \vartheta_{h}\right)=\left(f\left(\cdot, t^{m+\theta}\right), \vartheta_{h}\right), \forall \vartheta_{h} \in V_{h}, \\
& \left(u_{h}^{0}-u_{0}, \vartheta_{h}\right)=0, \forall \vartheta_{h} \in V_{h},
\end{aligned}
$$

where $0 \leq \theta \leq 1, u_{h}^{m}$ represents the approximation of $u\left(\cdot, t^{m}\right)$, and for the sake of simplicity, we write:

$$
u_{h}^{m+\theta}(x)=\theta u_{h}^{m+1}(x)+(1-\theta) u_{h}^{m}(x),
$$

The right hand side for problem $I$ is

$$
f\left(\cdot, t^{m+\theta}\right)=-\left(\left.\theta \frac{2 r_{t}+\beta}{r}\right|_{t=t^{m+1}}+\left.(1-\theta) \frac{2 r_{t}+\beta}{r}\right|_{t=t^{m}}\right),
$$

and for the problem $I I$ is

$$
f\left(\cdot, t^{m+\theta}\right)=-\left(\left.\theta \frac{\frac{4}{3} r_{t}+\beta}{r}\right|_{t=t^{m+1}}+\left.(1-\theta) \frac{\frac{4}{3} r_{t}+\beta}{r}\right|_{t=t^{m}}\right) .
$$


We define the bilinear form:

$$
a\left(u_{h}^{m+\theta}, \vartheta_{h}\right)=\left(\left(u_{h}^{m+\theta}\right)_{x},\left(\vartheta_{h}\right)_{x}\right)-\left(\frac{2 r_{x}\left(u_{h}^{m+\theta}\right)_{x}}{r}, \vartheta_{h}\right)+\left(\frac{2 r_{t}+\beta}{r} u_{h}^{m+\theta}, \vartheta_{h}\right)
$$

for problem $I$, and

$$
a\left(u_{h}^{m+\theta}, \vartheta_{h}\right)=\left(r^{\frac{2}{3}}\left(u_{h}^{m+\theta}\right)_{x},\left(\vartheta_{h}\right)_{x}\right)-\frac{4}{3}\left(\frac{2 r_{x}\left(u_{h}^{m+\theta}\right)_{x}}{r^{\frac{1}{3}}}, \vartheta_{h}\right)+\left(\frac{\frac{4}{3} r_{t}+\beta}{r} u_{h}^{m+\theta}, \vartheta_{h}\right)
$$

for the problem $I I$.

Note that as the our forward problem is non-linear, we do not have enough information for $f\left(\cdot, t^{m+1}\right)$, therefore we approximate $\left(f\left(\cdot, t^{m+1}\right)\right.$ by

$$
\left(f\left(\cdot, t^{m+1}\right)=\frac{2 \frac{r^{m+1}(\cdot)-r^{m}(\cdot)}{\Delta t}+\frac{D_{2}}{\int_{0}^{1} r^{m+1} c^{m} d x}}{r^{m+1}(\cdot)}\right.
$$

and

$$
f\left(\cdot, t^{m+1}\right)=\frac{\frac{4}{3} \frac{r^{m+1}(\cdot)-r^{m}(\cdot)}{\Delta t}+\frac{D_{2}}{\int_{0}^{1} r^{m+1} c^{m} d x}}{r^{m+1}(\cdot)}
$$

for problem $I$ and $I I$ correspondingly. Equivalently, (5.30) can be written as

$$
\begin{aligned}
& \left(v_{h}^{m+1}, \vartheta_{h}\right)+\Delta t a\left(v_{h}^{m+1}, \vartheta_{h}\right)=\Delta t\left(f\left(\cdot, t^{m+1}\right), \vartheta_{h}\right), \forall \vartheta \in V_{h}, \\
& \forall \vartheta_{h} \in V_{h} .
\end{aligned}
$$

Thus, given $u_{h}^{m}$, supposing that

$$
u_{h}^{m}=\sum_{j=1}^{J-2} \mu_{j}^{m} \Phi_{j}
$$

we will find $u_{h}^{m+1}=\sum_{j=1}^{J-2} \mu_{j} \Phi_{j}$ at the time level $t^{m+1}$, we have to solve a system of $J-2$ linear equations obtained after replacing $\vartheta_{h}$ in (5.38) by $\Phi_{1}, \Phi_{2}, \ldots, \Phi_{J-2}$

$$
B \mu=b,
$$

where $B$ is a $(J-2) \times(J-2)$ matrix:

$$
\begin{gathered}
B=\left[\begin{array}{ccccc}
b b_{1} & c c_{1} & 0 & \cdots & 0 \\
a a_{1} & b b_{2} & c c_{2} & \cdots & 0 \\
\cdots & \cdots & \cdots & \cdots & \cdots \\
0 & \cdots & \cdots & b b_{J-3} & c c_{J-3} \\
0 & \cdots & \cdots & a a_{J-2} & b b_{J-2}
\end{array}\right] \\
\mu=\left(\mu_{1}, \cdots, \mu_{J-2}\right), b=\left(b_{1}, \cdots, b_{J-2}\right)^{T} .
\end{gathered}
$$


The elements of the matrix $\mathrm{B}$ and the right hand sides $b$ are determined by

$$
\begin{aligned}
& b b_{i}=\frac{2 h}{3}+\frac{2 h}{3} \theta \Delta t \frac{2 r_{t}+\beta}{r}+2 \theta \frac{\Delta t}{h}, i=1, \cdots, J-3, \\
& b b_{J-2}=\frac{4 h}{3}+\frac{4 h}{3} \theta \Delta t \frac{2 r_{t}+\beta}{r}+\theta \frac{\Delta t}{h}-\theta \frac{\Delta t r_{x}}{r}, \\
& a a_{i}=\frac{h}{6}+\frac{h}{6} \theta \Delta t \frac{2 r_{t}+\beta}{r}-\theta \frac{\Delta t}{h}+\theta \frac{\Delta t r_{x}}{r}, i=1, \cdots, J-3, \\
& c c_{i}=\frac{h}{6}+\frac{h}{6} \theta \Delta t \frac{2 r_{t}+\beta}{r}-\theta \frac{\Delta t}{h}-\theta \frac{\Delta t r_{x}}{r}, i=1, \cdots, J-3, \\
& b_{1}=\left[\frac{2 h}{3}+\frac{2 h}{3} \Delta t(1-\theta) \frac{2 r_{t}+\beta}{r}-2(1-\theta) \frac{\Delta t}{h}\right] \mu_{1}^{m}+ \\
&+\left[\frac{h}{6}-\frac{h}{6}(1-\theta) \Delta t \frac{2 r_{t}+\beta}{r}+(1-\theta) \frac{\Delta t}{h}+(1-\theta) \frac{\Delta t r_{x}}{r}\right] \mu_{2}^{m}-\Delta t h\left(2 r_{t}+\beta\right)\left(\frac{\theta}{r_{1}^{m+1}}+\frac{1-\theta}{r_{1}^{m}}\right), \\
& b_{i}=\left[\frac{h}{6}-\frac{h}{6}(1-\theta) \Delta t \frac{2 r_{t}+\beta}{r}+(1-\theta) \frac{\Delta t}{h}-(1-\theta) \frac{\Delta t r_{x}}{r}\right] \mu_{i-1}^{m}+ \\
&\left.+\left[\frac{2 h}{3}-\frac{2 h}{3}(1-\theta) \frac{2 r_{t}+\beta}{r}-2(1-\theta) \frac{\Delta t}{h}+(1-\theta)\right) \frac{\Delta t r_{x}}{r}\right] \mu_{i}^{m}+ \\
&\left.+\left[\frac{h}{6}-\frac{h}{6}(1-\theta) \Delta t \frac{2 r_{t}+\beta}{r}+(1-\theta) \frac{\Delta t}{h}+(1-\theta)\right) \frac{\Delta t r_{x}}{r}\right] \mu_{i+1}^{m}-h \Delta t\left(2 r_{t}+\beta\right)\left(\frac{\theta}{r_{i}^{m+1}}+\frac{1-\theta}{r_{i}^{m}}\right), i=1, \cdots, J-3, \\
&+
\end{aligned}
$$

for the problem $I$ and

$$
\begin{aligned}
& b b_{i}=\frac{2 h}{3}+\frac{2 h}{3} \theta \Delta t \frac{\frac{4}{3} r_{t}+\beta}{r}+\theta r^{\frac{2}{3}} \frac{2 \Delta t}{h}, i=1, \cdots, J-3, \\
& b b_{J-2}=\frac{4 h}{3}+\frac{4 h}{3} \theta \Delta t \frac{\frac{4}{3} r_{t}+\beta}{r}+\theta r^{\frac{2}{3}} \frac{\Delta t}{h}-\frac{2}{3} \theta \frac{\Delta t r_{x}}{r^{\frac{1}{3}}} \\
& a a_{i}=\frac{h}{6}+\frac{h}{6} \theta \Delta t \frac{\frac{4}{3} r_{t}+\beta}{r}-\theta r^{\frac{2}{3}} \frac{\Delta t}{h}+\frac{2}{3} \theta \frac{\Delta t r_{x}}{r^{\frac{1}{3}}}, i=2, \cdots, J-2, \\
& c c_{i}=\frac{h}{6}+\frac{h}{6} \theta \Delta t \frac{\frac{4}{3} r_{t}+\beta}{r}-\theta r^{\frac{2}{3}} \frac{\Delta t}{h}+\frac{2}{3} \theta \frac{\Delta t r_{x}}{r^{\frac{1}{3}}}, i=1, \cdots, J-3,
\end{aligned}
$$




$$
\begin{aligned}
& b_{1}=\left[\frac{2 h}{3}+\frac{2 h}{3} \Delta t(1-\theta) \frac{\frac{4}{3} r_{t}+\beta}{r}-2(1-\theta) r^{\frac{2}{3}} \frac{\Delta t}{h}\right] \mu_{1}^{m}+ \\
& {\left[\frac{h}{6}-\frac{h}{6}(1-\theta) \Delta t \frac{\frac{4}{3} r_{t}+\beta}{r}+(1-\theta) r^{\frac{2}{3}} \frac{\Delta t}{h}+\frac{2}{3}(1-\theta) \frac{\Delta t r_{x}}{r^{\frac{1}{3}}}\right] \mu_{2}^{m}+-\Delta t h\left(\frac{4}{3} r_{t}+\beta\right)\left(\frac{\theta}{r_{1}^{m+1}}+\frac{1-\theta}{r_{1}^{m}}\right),} \\
& b_{i}=\left[\frac{h}{6}-\frac{h}{6}(1-\theta) \Delta t \frac{\frac{4}{3} r_{t}+\beta}{r}+(1-\theta) r^{\frac{2}{3}} \frac{\Delta t}{h}-\frac{2}{3}(1-\theta) \frac{\Delta t r_{x}}{r^{\frac{1}{3}}}\right] \mu_{i-1}^{m}+ \\
& \left.+\left[\frac{2 h}{3}-\frac{2 h}{3}(1-\theta) \frac{\frac{4}{3} r_{t}+\beta}{r}-2(1-\theta) r^{\frac{2}{3}} \frac{\Delta t}{h}+\frac{2}{3}(1-\theta)\right) \frac{\Delta t r_{x}}{r^{\frac{1}{3}}}\right] \mu_{i}^{m}+ \\
& \left.+\left[\frac{h}{6}-\frac{h}{6}(1-\theta) \Delta t \frac{\frac{4}{3} r_{t}+\beta}{r}+(1-\theta) r^{\frac{2}{3}} \frac{\Delta t}{h}+\frac{2}{3}(1-\theta)\right) \frac{\Delta t r_{x}}{r^{\frac{1}{3}}}\right] \mu_{i+1}^{m}- \\
& -h \Delta t\left(\frac{4}{3} r_{t}+\beta\right)\left(\frac{\theta}{r_{i}^{m+1}}+\frac{1-\theta}{r_{i}^{m}}\right), i=1, \cdots, J-3, \\
& \left.b_{J-2}=\left[\frac{h}{6}-\frac{h}{6}(1-\theta) \Delta t \frac{\frac{4}{3} r_{t}+\beta}{r}+(1-\theta) r^{\frac{2}{3}} \frac{\Delta t}{h}-\frac{2}{3}(1-\theta)\right) \frac{\Delta t r_{x}}{r^{\frac{1}{3}}}\right] \mu_{J-3}^{m}+ \\
& \left.+\left[\frac{4 h}{3}-\frac{4 h}{3}(1-\theta) \Delta t \frac{\frac{4}{3} r_{t}+\beta}{r}-(1-\theta) r^{\frac{2}{3}} \frac{\Delta t}{h}+\frac{2}{3}(1-\theta)\right) \frac{\Delta t r_{x}}{r^{\frac{1}{3}}}\right] \mu_{J-2}^{m}-\frac{3 h}{2} \Delta t\left(\frac{4}{3} r_{t}+\beta\right)\left(\frac{\theta}{r_{J-2}^{m+1}}+\frac{1-\theta}{r_{J-2}^{m}}\right)
\end{aligned}
$$

for the problem $I I$.

The Pseudo codes of the FEM for the forward problem is presented as the Algorithm 5.1.

\subsubsection{Comparison between the implicit FDM and the FEM.}

In [9], the authors compare the difference between the implicit FDM and the explicit FDM. They carried out the experiment using electrolyte $D M S O^{L i^{+}}$, considering the pore clogging occurs when the pore radius goes below than 0.1 . The two above methods show very slightly difference: radius, concentration and the deviation between them is below $0.001 \%$, where the deviation is defined by:

$$
\sigma_{V_{\text {free }}}=\frac{\left|V_{\text {free }}^{\text {exp }}-V_{\text {free }}^{\text {imp }}\right|}{V_{\text {free }}^{\text {exp }}} .
$$

However, the run time for the explicit FDM is much longer that the one for the implicit FDM. We adapt the table from [9]:

Following [9], we define the stability of a numerical method for this problem by the considering the concentration $c(t, x)$ at every time step $t^{n}=n \Delta t$. The stability is held if $c \in[0,1]$ when $r(t, x)>0.1$. 


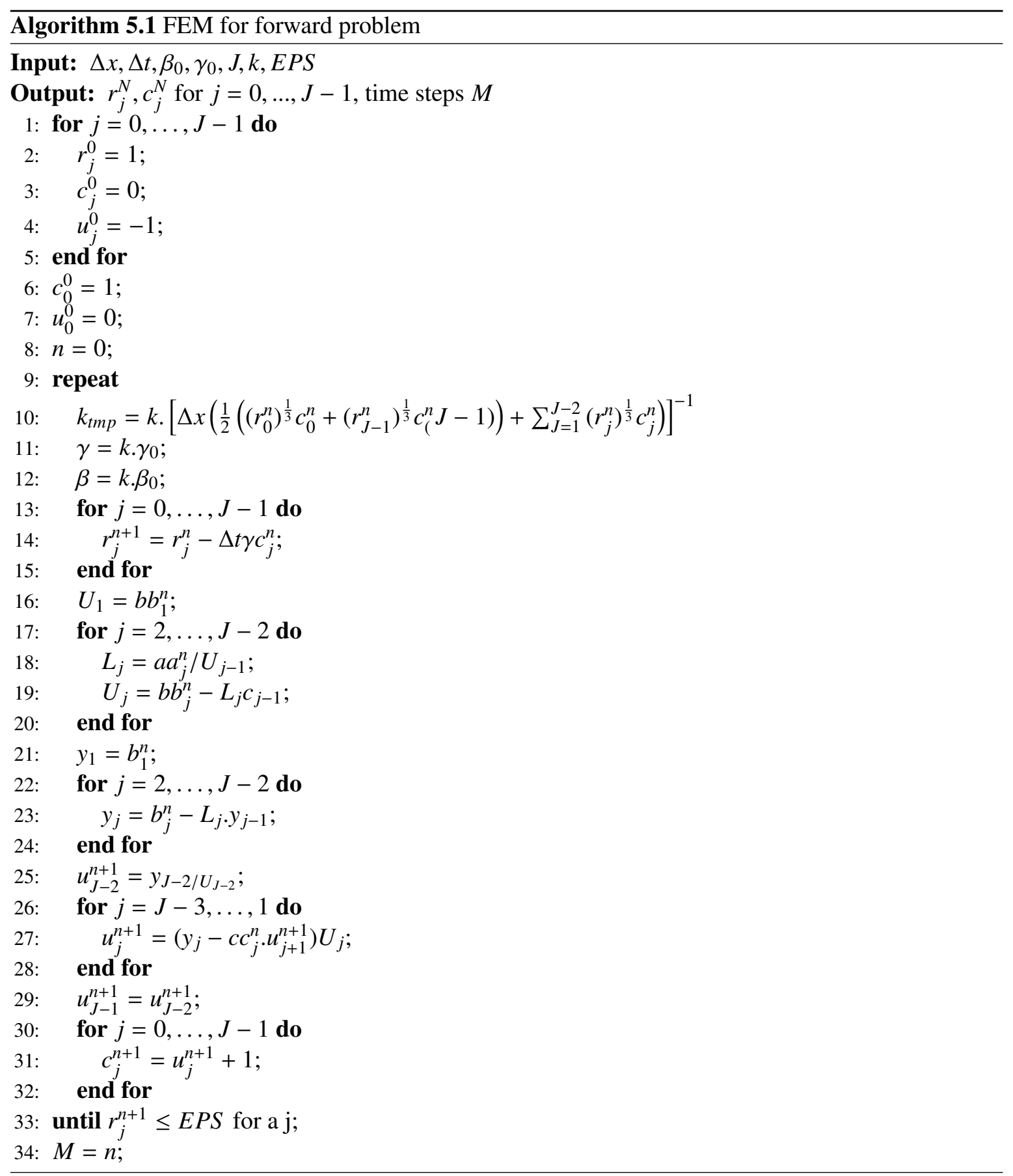




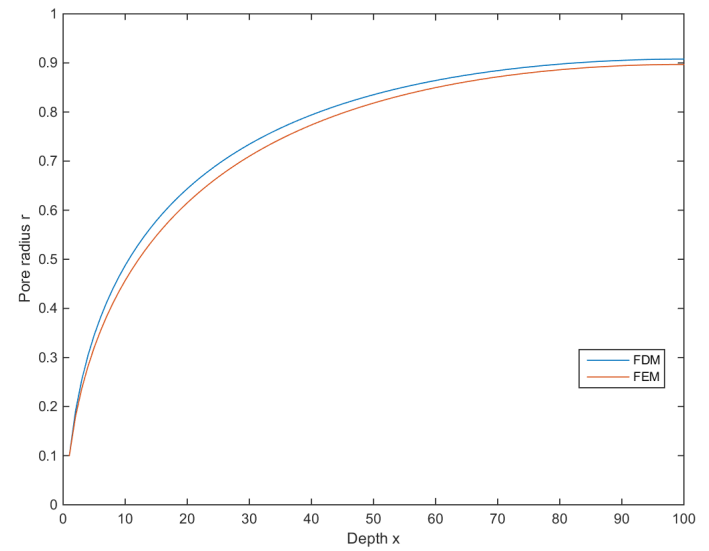

(a) The pore radii

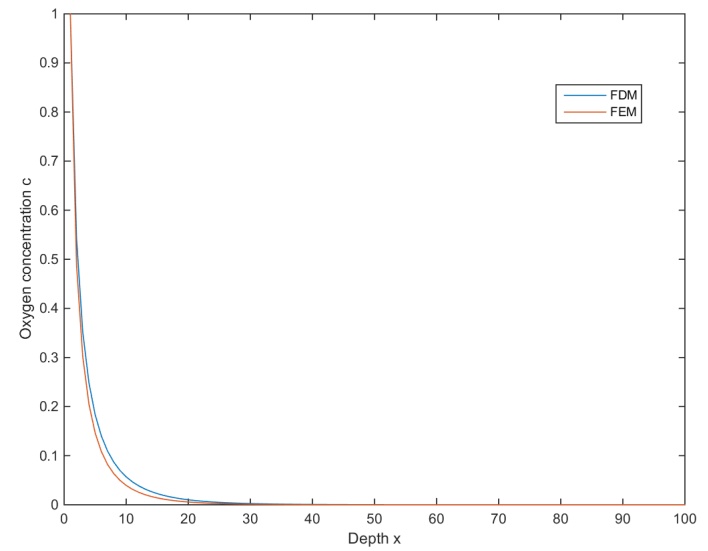

(b) The concentration of oxygen

Figure 5.4: The pore radii and the concentration of oxygen inside pore before pore clogging with $J=N=100$ solving with FDM and FEM

\begin{tabular}{rrrrrrrr}
\hline$J$ & \multicolumn{2}{c}{$(\Delta t)^{-1}$} & \multicolumn{2}{c}{$V_{\text {free }} / \%$} & $\sigma_{V_{\text {free }} / \%}$ & \multicolumn{2}{c}{$t_{\text {run }} / \mathrm{s}$} \\
& implicit & explicit & implicit & explicit & & implicit & explicit \\
\hline 100 & 100 & 24000 & 61.6157 & 61.6152 & 0.0008 & 20.10 & 4762 \\
200 & 200 & 92000 & 61.7067 & 61.7063 & 0.0006 & 82.01 & 37165 \\
300 & 300 & 201000 & 61.7335 & 61.7332 & 0.0005 & 182.91 & 115751 \\
400 & 400 & 352000 & 67.7460 & 61.7457 & 0.0004 & 326.21 & 268083 \\
\hline
\end{tabular}

Figure 5.5: Free pore volume, relative deviation and runtime for the implicit and explicit FDM

The Table in the Figure 5.5 shows the free volume after pore clogging, the runtime in different time steps as well as the stability in two these methods in spacial discretion of $J=100$ and for the problem (II). The FEM we use here is in case of $\theta=1$ and $f\left(\cdot, t^{m}\right)$ in the right hand side in stead of $f\left(\cdot, t^{m+1}\right)$ in (5.37). The deviation in this Table is defined by:

$$
\sigma_{V_{\text {free }}}^{*}=\frac{\left|V_{\text {free }}^{i m p}-V_{\text {free }}^{F E M}\right|}{V_{\text {free }}^{i m p}} .
$$


5.2. Some remarks on the Greedy algorithm in Problem $I I_{b}$ and $I_{b}$ using FDM.

\begin{tabular}{ccccccc}
\hline$\Delta t$ & $N=(\Delta t)^{-1}+1$ & \multicolumn{2}{c}{$V_{\text {free }} / \%$} & $\sigma_{V_{\text {free }}}^{*} / \%$ & \multicolumn{2}{c}{$t_{\text {run }} / s$} \\
& & FEM & FDM implicit & & FEM & FDM implicit \\
\hline 0.010101 & 100 & 58.9922 & 61.6157 & 4.2578 & 17.0621 & 10.498 \\
0.020408 & 50 & 58.9929 & 61.6163 & 4.2576 & 8.46296 & 5.49169 \\
0.025641 & 40 & 58.9932 & 61.6166 & 4.2576 & 6.4961 & 4.25776 \\
0.034482 & 30 & 58.9938 & 61.6591 & 4.3226 & 5.1977 & 3.1829 \\
0.052631 & 20 & 58.995 & not stable & & 3.55292 & not stable \\
0.111111 & 10 & 58.9986 & not stable & & 1.57534 & not stable \\
0.142857 & 8 & 59.0013 & not stable & & 1.34791 & not stable \\
0.2 & 6 & 59.0052 & not stable & & 1.01438 & not stable \\
0.333333 & 4 & 59.0654 & not stable & & 0.717149 & not stable \\
1 & 2 & 58.9986 & not stable & & 0.222847 & not stable \\
2 & 1.5 & 59.1416 & not stable & & 0.118924 & not stable \\
5 & 1.2 & 59.4254 & not stable & & 0.0469593 & not stable \\
\hline
\end{tabular}

Table 5.1: Free volume and runtime for the implicit FDM and FEM with spacial discretion of $J=100$

Concern the continuous catalyst positioning model cat(x)(Problems $\left.I_{a}, I I_{a}\right)$ and the continuous catalyst positioning model cat $(\mathrm{t}, \mathrm{x})$ (Problems $I_{b}$ and $I I_{b}$ ) in this Thesis, we care about the runtime in order to implement the different optimization methods such as sensitivity system and adjoint methods. For this, FEM is a potential method. In this table, the deviation shows that there is a certain difference between the two solution of the two methods (deviation is below than 4.4\%) with $N=100,50,40,30$, with $\Delta t=0.010101,0.020408,0.015641,0.034482$, relatively. The runtime of FDM method is below than the runtime of FEM in these four $\Delta t$, as the computation of the coefficients of the matrix $B$ consumes more time for the ones of the matrix $A$. However, for the other greater $\Delta t$ in the Table 5.5, the implicit FDM is not stable, while the FEM still shows an acceptable solution.

\subsection{Some remarks on the Greedy algorithm in Problem $I I_{b}$ and $I_{b}$ using FDM.}

According to [8] the greedy algorithm in the catalyst positioning problem for Lithium/air battery is the step-by-step selection of the optimal position of catalyst subsequently based on minimizing an objective function, in this case it is the free volume of the pore inside the cathode before pore clogging:

$$
V_{\text {free }}^{n}=\Delta x\left[\frac{1}{2}\left(\left(r_{0}^{n}\right)^{2}+\left(r_{J-1}^{n}\right)^{2}\right)+\sum_{j=1}^{J-2}\left(r_{j}^{n}\right)^{2}\right] .
$$

This $V_{\text {free }}^{n}$ is calculated by the trapezoidal rule.

The Greedy algorithm normally does not give a global minimum, as it does not solve the optimal problem globally on the whole original domain [2]. In [8], the authors choose the minimal node in 


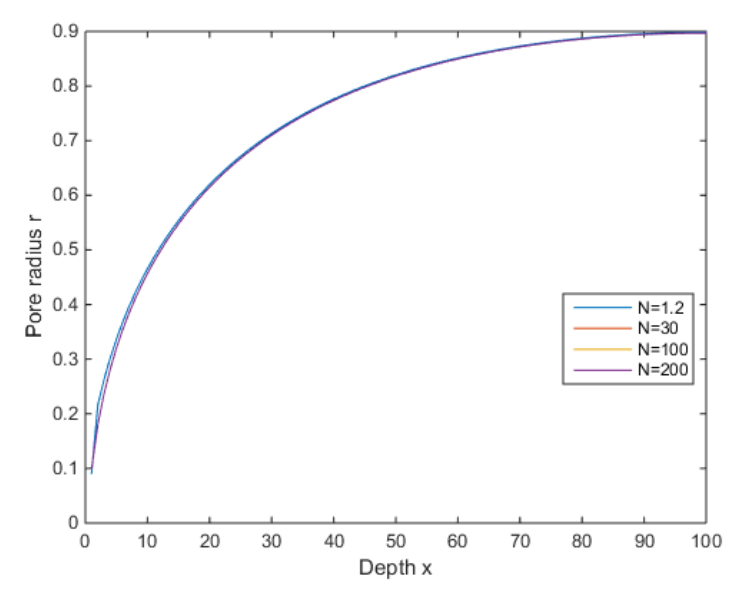

(a) The pore radii

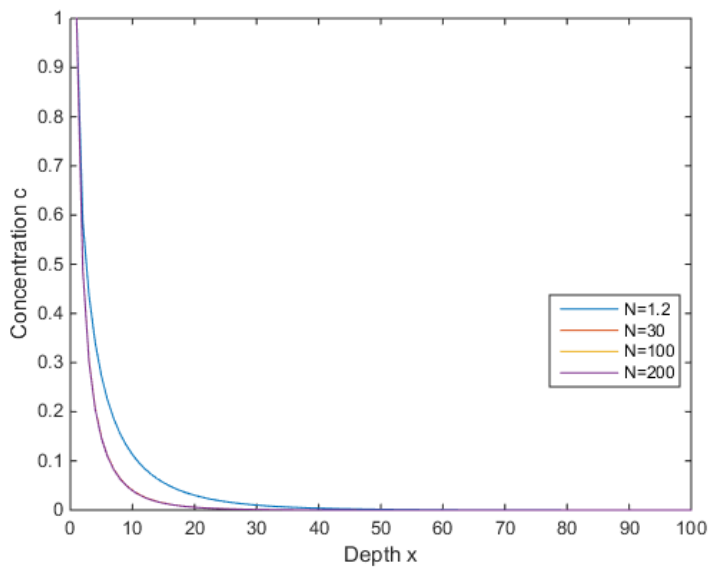

(b) The concentration of oxygen inside the pore

Figure 5.6: The pore radii and the concentration of oxygen inside pore before pore clogging with $J=100$ and different $\mathrm{N}$ in Problem $I$

the first step by checking the whole $J$ possible position of the catalyst. Remembering this node, they continue to improve the algorithm in the next step by checking the whole combinations of the previous minimal node with one of the $(J-1)$ other notes to select the next optimal position of catalyst. They stops this process when $V_{\text {free }}^{n}>V_{\text {free }}^{n-1}$ in the $n$-th iteration. However, we would like to recommend to continue this checking process after this $n$-th state to globalize the algorithm. We let $n$ run from 1 to $J$ and then selecting the "global" greedy algorithm solution, which is corresponding to the minimum value $\min _{n} V_{\text {free }}^{n}, n=1, \cdots, J$.

We check with electrolyte DMSO, discretion of space is $J=100$, threshold of $4 \%$ then we get the first local minimum at the 33-th step with $V_{\text {free }}^{37}=24,61 \%$, and the second local minimum at the 47-th iteration with $V_{\text {free }}^{37}=23,46 \%$. In the Figure 5.11a, the graph of optimal the numbers of catalyst with the minimal $V_{\text {free }}$ volume. We can see from this graph that number of 47 is the better optimal number in this experiment.

Figure 5.11a shows the comparison between the two state of the pore before pore clogging. Radius of the pore at the 33-th iteration (red line) is lower than the radius of the pore at 47-th iteration (blue line) at the center of the pore and they are slightly different at the end of the pore, where the diffusion limited side sited.

Figure 5.10 expresses the distribution of the optimal catalyst number of 47, that is the global greedy algorithm solution in this case.

We also present an experiment for Problem $I_{b}$. Figure 5.11 shows the evolutions of pore radii and concentration of oxygen along the pore over time with the optimal catalyst number of 41 , which is illustrated in the Figure 5.12. In this experiment, we take $J=N=100$, threshold of $4 \%$. 


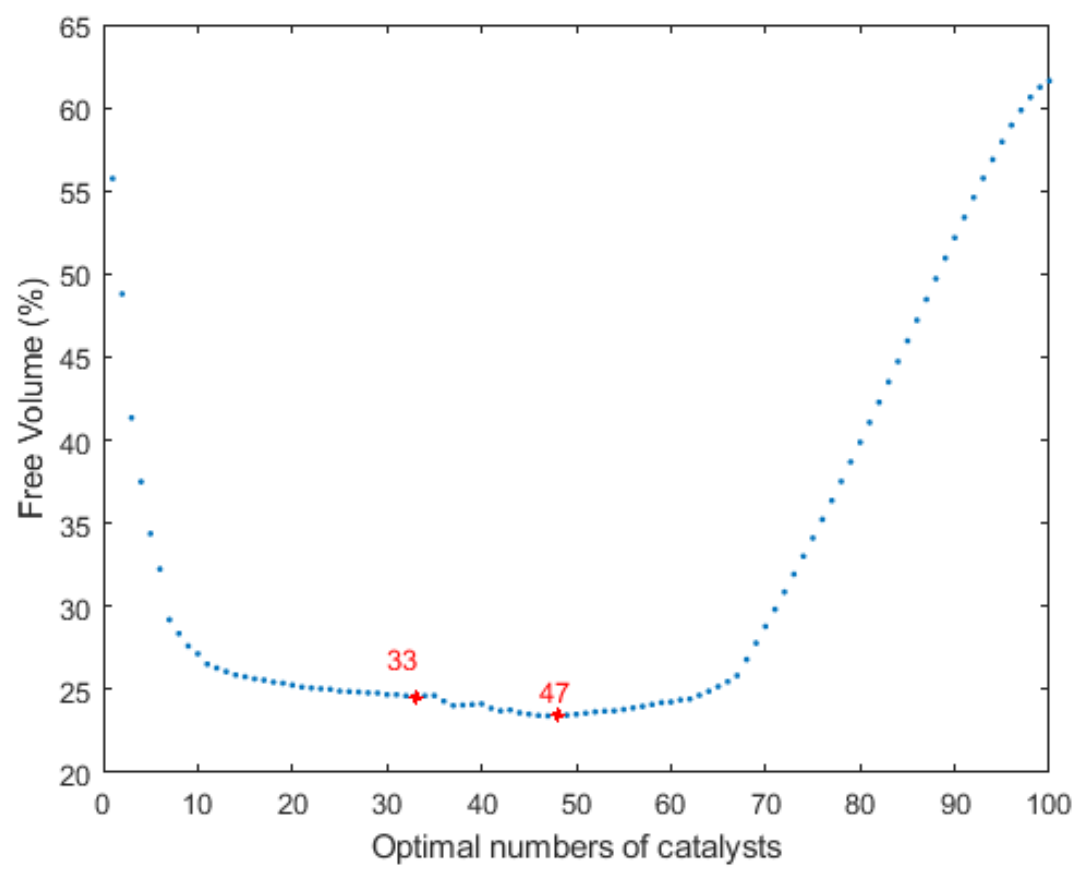

Figure 5.7: The optimal numbers of catalysts from 1 to 100 by Greedy algorithm in Problem $I I_{b}$

\subsection{The continuous positioning}

As we mentioned in Chapter Introduction of this Thesis, the authors in [9] implements a simple propagation process of the catalytic function to the neighbourhood using a binary function. This function either allows depositing catalyst development to the sites or prevents it. This discrete model gives us the first understandings about the binary distributed catalyst positioning and very good comparison of the effect of different electrolytes concerning to optimizing the capacity of the batteries. However, the natural question is Can we model a more visual catalytic function with a clearer geometrical features in order to acknowledge the distribution of catalyst inside the pore? We wish to aim at the more complex structured catalytic functions:

- Cat(x) Model with fractional function that characterizing the presence of catalyst at the sites, as well as the amount of catalyst at certain positions.

- $\operatorname{Cat}(\mathrm{t}, \mathrm{x})$ Model, which also is also a fractional catalytic function based on time dependent growth kinetics.

The analytic research on the two above models have been presented in Chapters 3 and 4 . In the next Subsections, we would like to show some numerical simulations and comparisons of these two Models.

We first transform the distribution of catalyst sites in the discrete catalyst model using in Greedy method to the continuous one using for the two continuous catalyst models cat( $\mathrm{x})$ and $\operatorname{cat}(\mathrm{t}, \mathrm{x})$. For this, we will use the convolution to smoothen the catalytic function in the following subsection. 


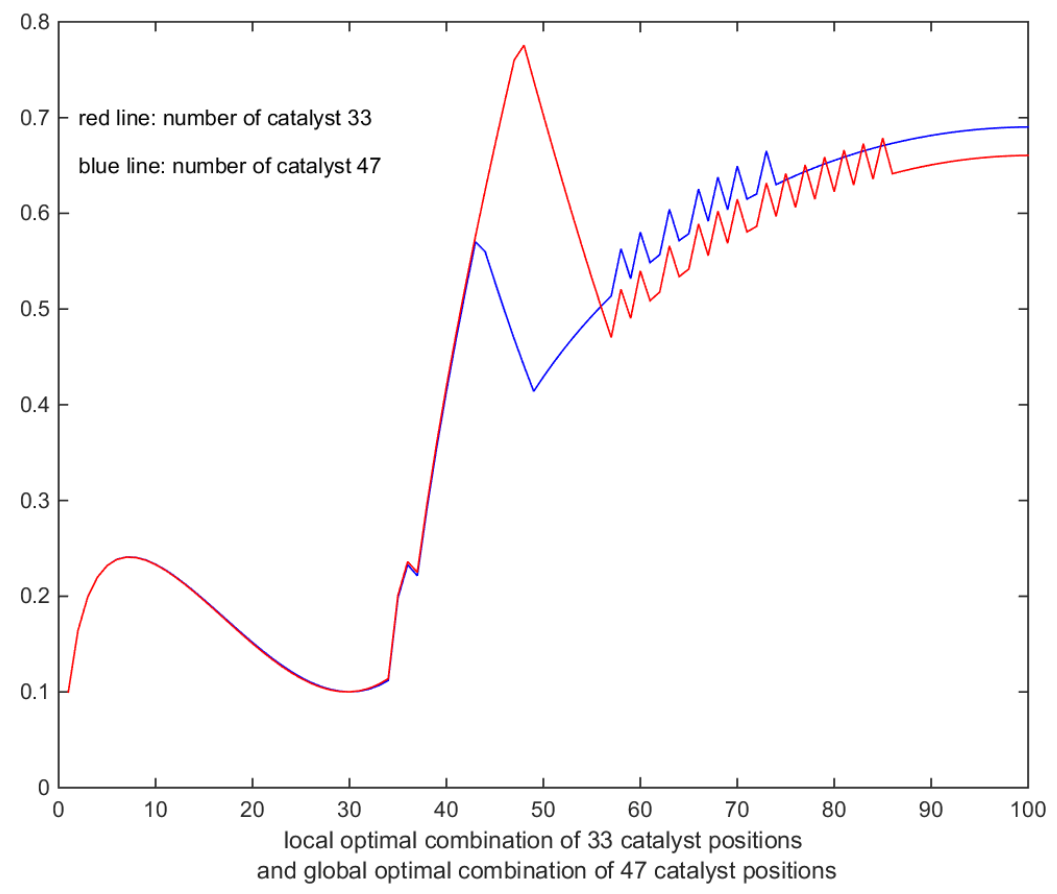

(a) The radii of pore at the optimal number of catalyst before pore closing

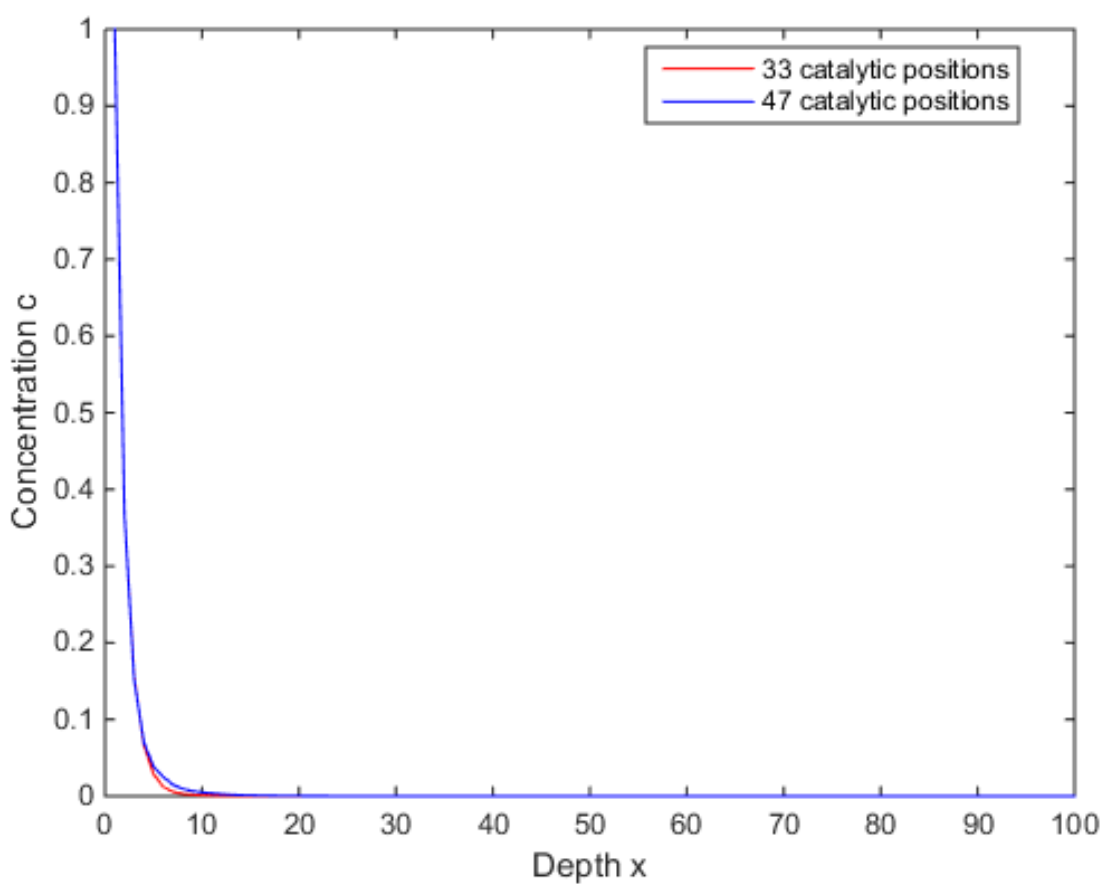

(b) The concentration of oxygen inside the pore with the two optimal numbers of catalyst before pore clogged

Figure 5.8: Comparison of radii and concentration inside the pore with two optimal numbers of catalyst before pore clogged by Greedy algorithm in Problem $I I_{b}$ 


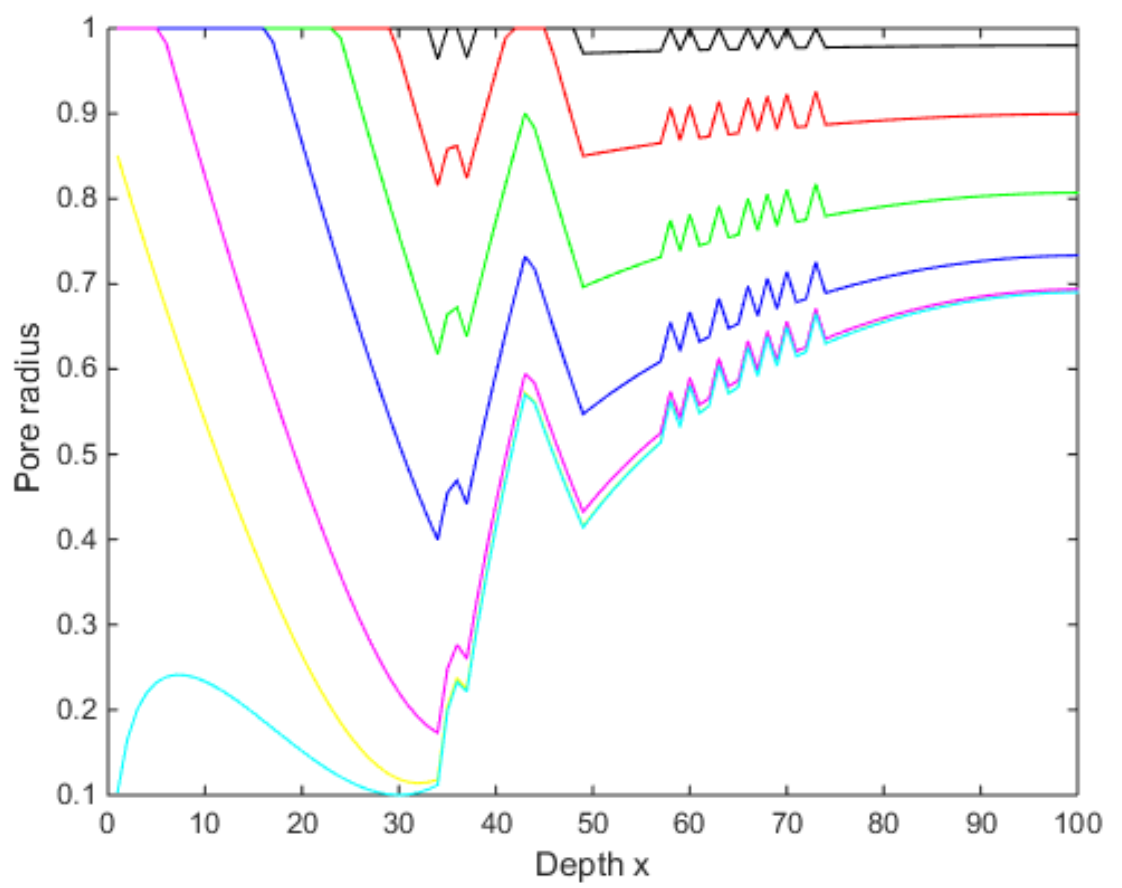

(a) The $\mathrm{Li}_{2} \mathrm{O}$ growth profile or the radii of pore over time.

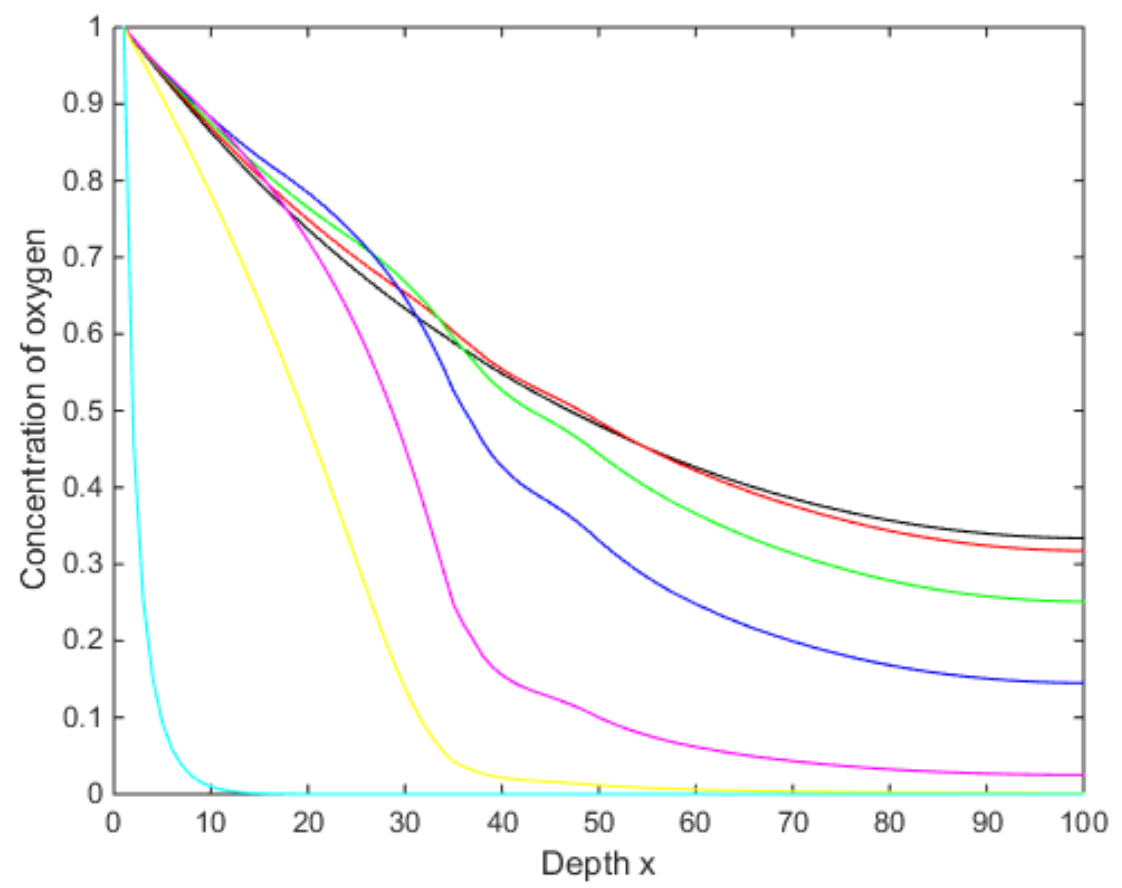

(b) The concentration of oxygen inside the pore over time.

Figure 5.9: radii and concentration inside the pore with 47 optimal number catalyst by Greedy algorithm in Problem $I I_{b}$. 


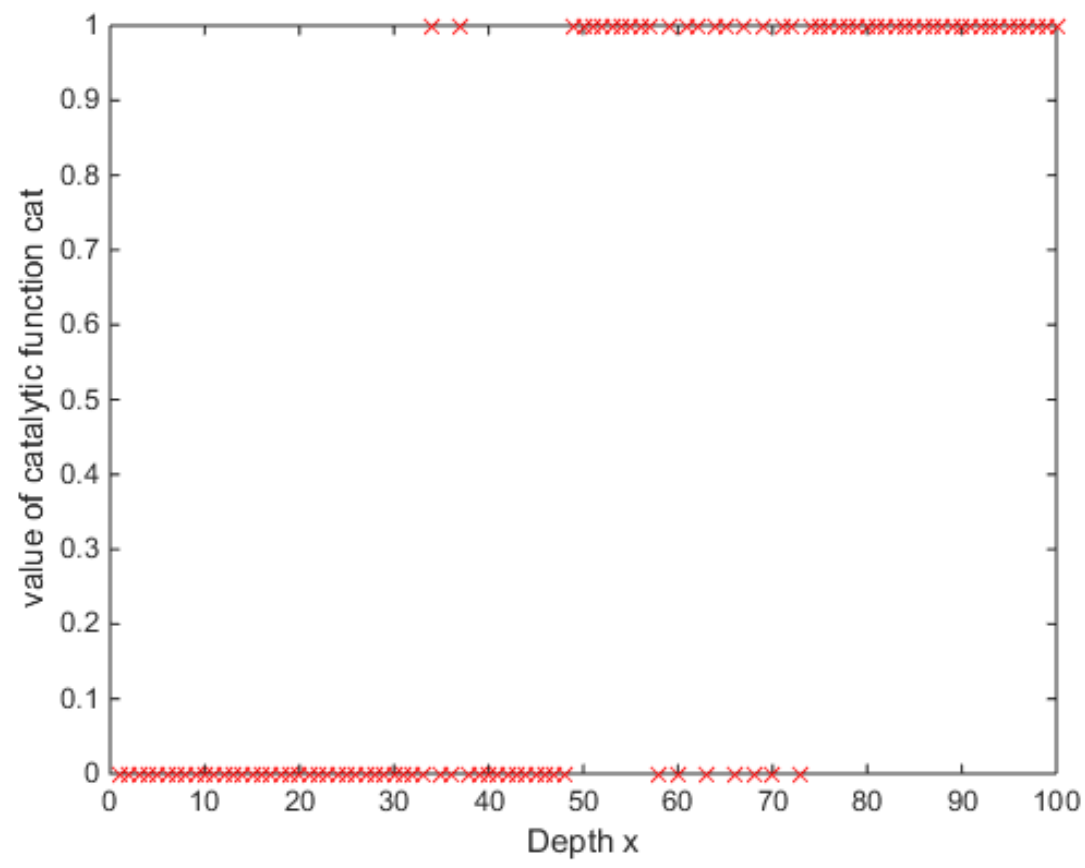

Figure 5.10: The distribution of optimal number of catalysts of 47 catalyst positions

\subsubsection{Transformation of discrete catalytic functions to smooth catalytic functions}

Suppose that $\operatorname{cat}_{d i s}(x) \in L^{\infty}(\Omega)$ is a discrete catalytic function such that its values $\operatorname{cat}_{d i s}\left(x_{j}\right)$ at $x_{j}=$ $j \Delta x$, where $j=0, \cdots, J-1, x_{0}=0, x_{J-1}=1$ could be 0 or 1 . We defined the extended discrete catalytic function $\mathbb{E}_{c a t}(x)$ of cat $_{\text {dis }}(x)$ by:

$$
\mathbb{E}_{c a t}\left(x_{j}\right)=\left\{\begin{array}{l}
\operatorname{cat}_{d i s}\left(x_{j}\right), \text { if } j=1, \cdots, J-2, \\
\operatorname{cat}_{d i s}\left(x_{J-1}\right), \text { if } j=J-1, \cdots, J-1+m,
\end{array}\right.
$$

where $m \in \mathbb{N}$.

The smooth transformation $\operatorname{cat}_{\text {smooth }}(x)$ of $\operatorname{cat}_{\text {dis }}(x)$ is now defined formally on $[0,1]$ as follows

$$
\operatorname{cat}_{\text {smooth }}(x)=\left.\left(\alpha_{\text {trans }} \int_{0}^{1+\sigma_{\text {trans }}} \mathbb{E}_{\text {cat }}(y) G(x-y) d x\right)\right|_{[0,1]},
$$

where $G(x-y)$ is normal Gaussian distribution at center y, $\alpha_{\text {trans }}$ is some positive constant and $\sigma_{\text {trans }} \geq$ $0, \sigma_{\text {trans }}=m \Delta x$.

Remark 5.1 We choose $\alpha_{\text {trans }}$ such that the values of cat smooth $(x)$ at $x_{j}=j \delta x$, where $j=0, \cdots, J-1$ are the real values from 0 to 1 .

Example of transformation: are described in Figure 5.13 and Figure 5.14 for two cases: $n=0$ and $m=7$. 


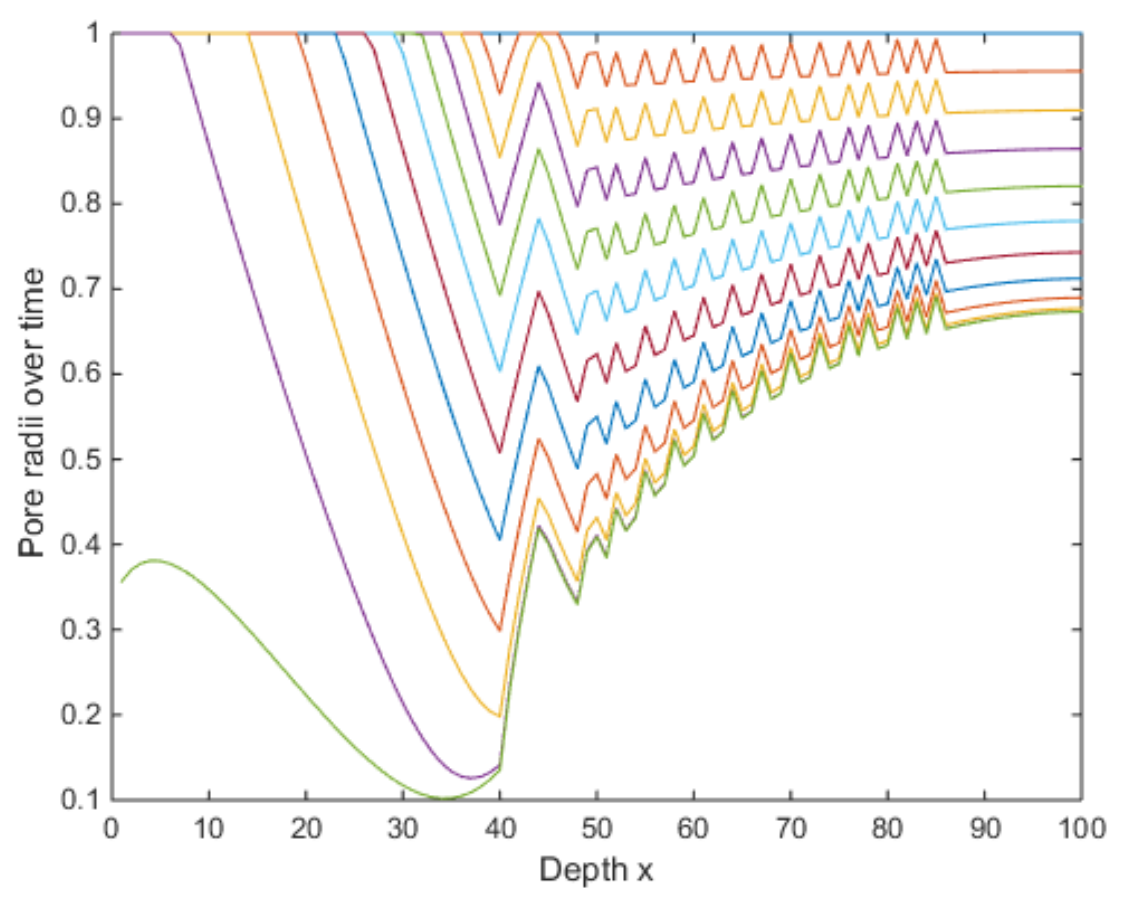

(a) The $\mathrm{Li}_{2} \mathrm{O}$ growth profile or the radii of pore over time.

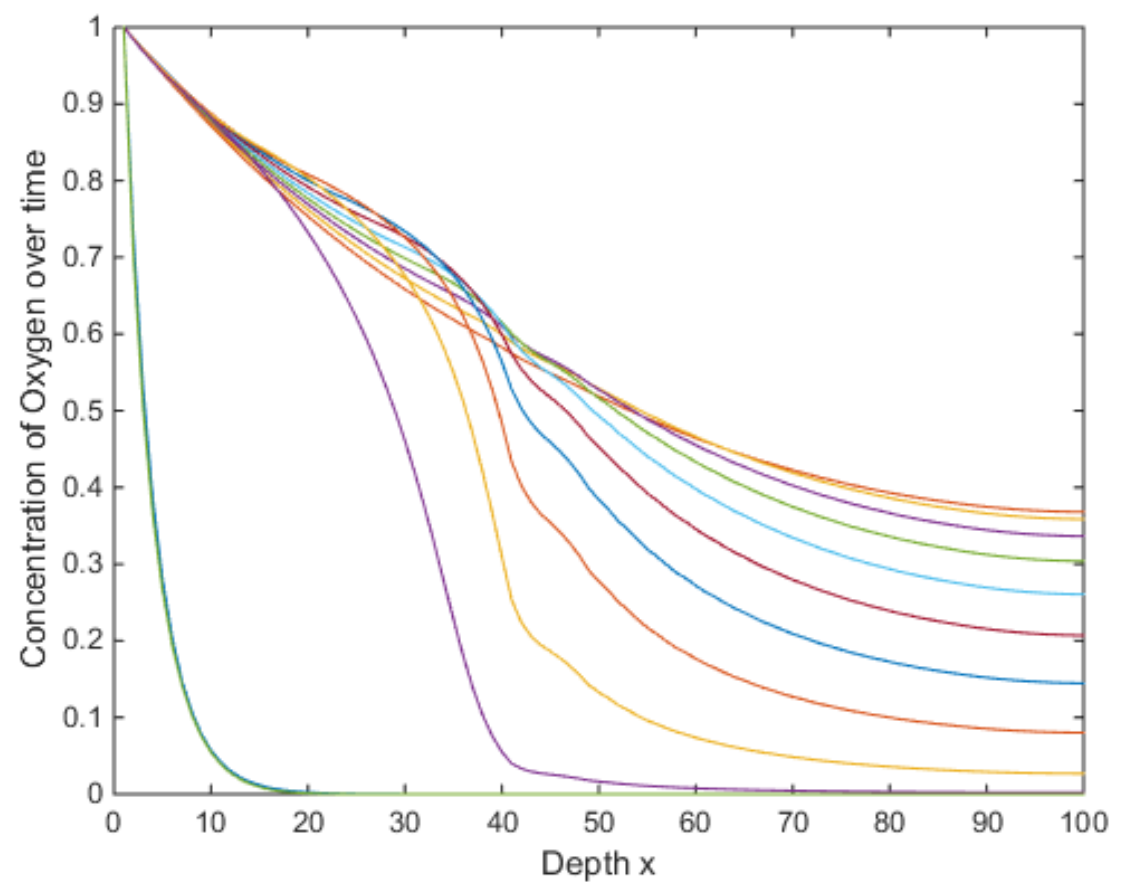

(b) The concentration of oxygen inside the pore over time.

Figure 5.11: radii and concentration inside the pore i line plotting with 41 optimal number catalyst by Greedy algorithm in Problem $I_{b}$. 


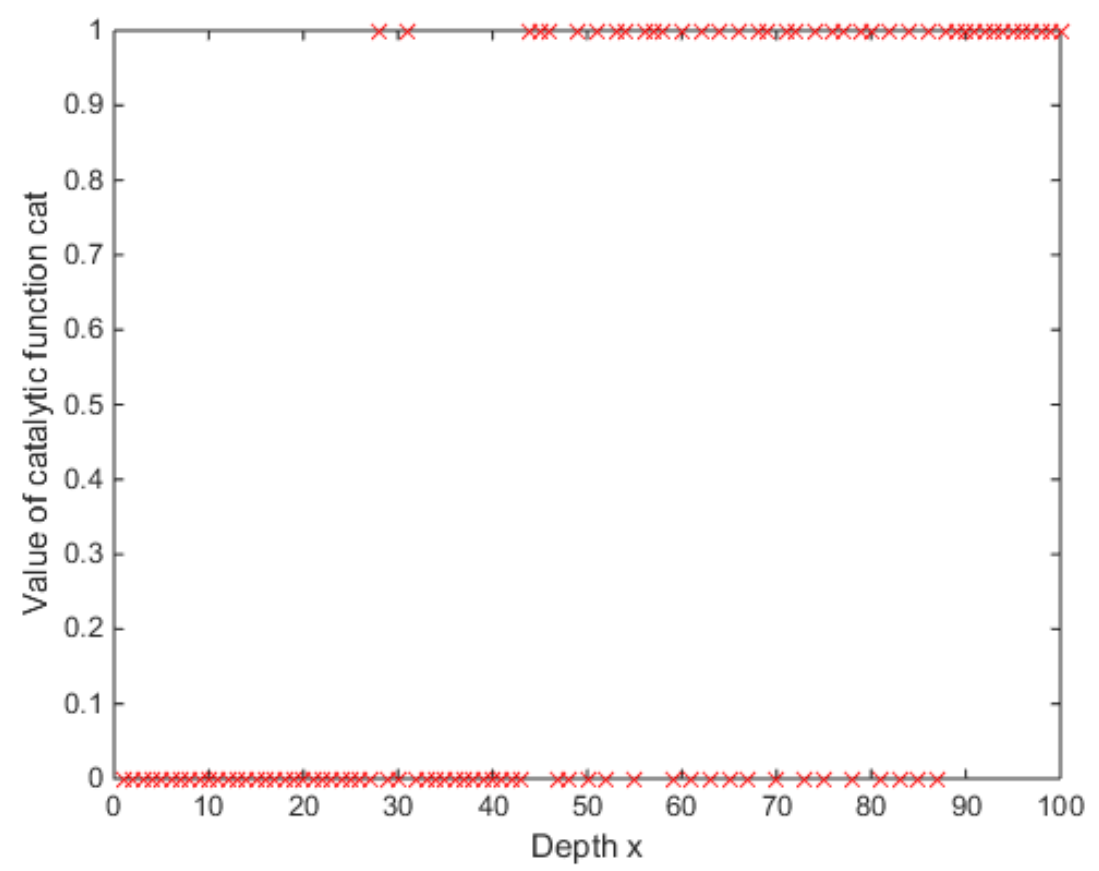

Figure 5.12: The distribution of optimal number of catalysts of 41 catalyst positions in Problem $I_{b}$

\subsection{2 $\operatorname{cat}(\mathbf{x})$ model (Problems $I_{a}$ and $\left.I I_{a}\right)$}

The catalytic function is defined as a function in $W^{1, \infty}(\Omega)$ is discretized via a function cat $_{j}, j \in$ $[0, \cdots, J-1]$, where $\operatorname{cat}_{j}=\operatorname{cat}\left(x_{j}\right) \in[0, \cdots, 1]$. The increasing pore radius $\mathrm{r}$ then is written by:

$$
r_{j}^{n+1}=r_{j}^{n}-\Delta t \gamma c_{j}^{n} c a t_{j}, \text { where } j=0, \cdots, J-1, n=0,1, \cdots
$$

The continuous catalyst positioning model cat(x) with the catalytic function taken fractional values from 0 to 1 allows us to simulate a smooth growth of $\mathrm{Li}_{2} \mathrm{O}_{2}$ along the active site in the cathode surface, once we choose smooth values for catalytic function. In this model, the catalyst function always keeps all its value with time, therefore, with the site where the the catalyst function with the value of 0 , the radius will not be changed, even in the oxygen entering side.

\subsection{3 cat $(\mathbf{t}, \mathbf{x})$ model (Problems $I_{b}$ and $\left.I I_{b}\right)$}

The cat $(\mathrm{t}, \mathrm{x})$ Model is inspired by the model of growth of $\mathrm{Li}_{2} \mathrm{O}_{2}$ from the Thesis of Tatjiana Dabrowski [9] using the Greedy Algorithm. Once the threshold is reached, the growth profile is enhanced to start also around the neighbourhood sites. There are circumstances occurred in the nature like bacteria growth or kinetics growth. Different thresholds $R_{0}$ or different $\alpha_{0}$ in (1.25) characterize different kind of growths. 


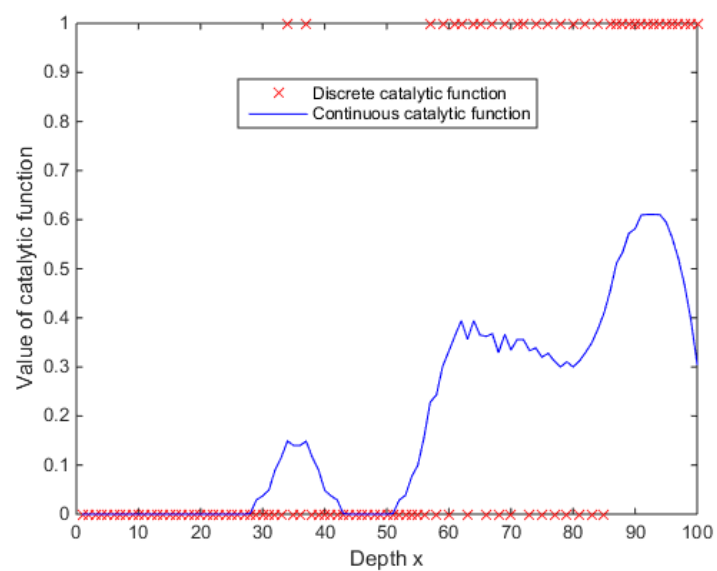

(a) The local optimal catalytic function (number of catalyst $=33$ ) by Greedy algorithm before pore reaches to 0.1 , $\mathrm{J}=100$

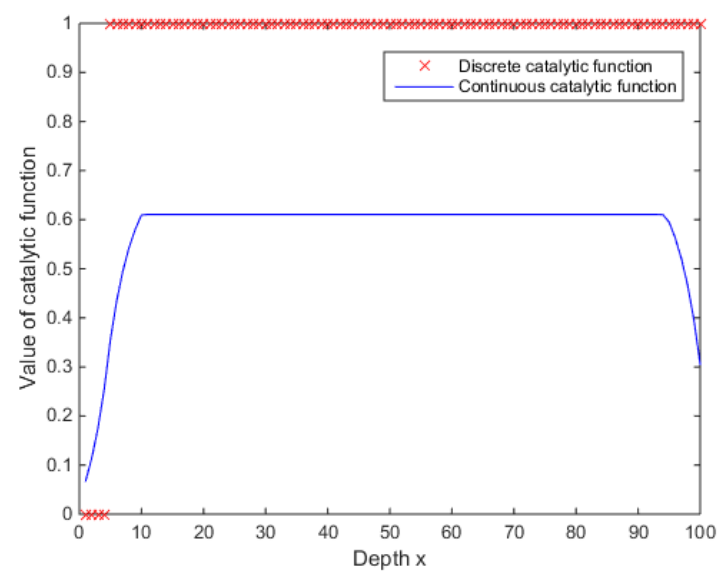

(b) The local optimal catalytic function by Greedy algorithm before pore reaches to $0.86, \mathrm{~J}=100$

Figure 5.13: The transformation from the local optimal discrete catalytic functions by Greedy algorithm in Problem $I I_{b}$ before pore reaches to 0.1 and 0.86 to continuous ones, with $\sigma_{\text {trans }}=0$ in (5.49)

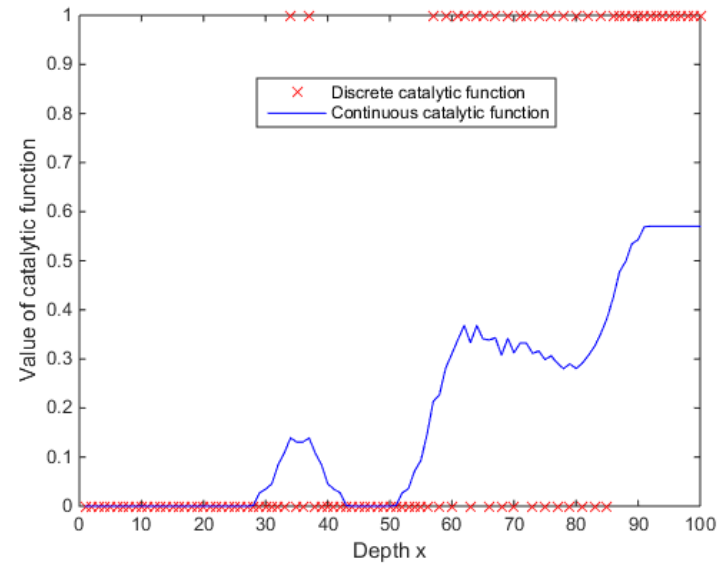

(a) The local optimal catalytic function (number of catalyst $=33$ ) by Greedy algorithm before pore reaches to 0.1 , $\mathrm{J}=100$

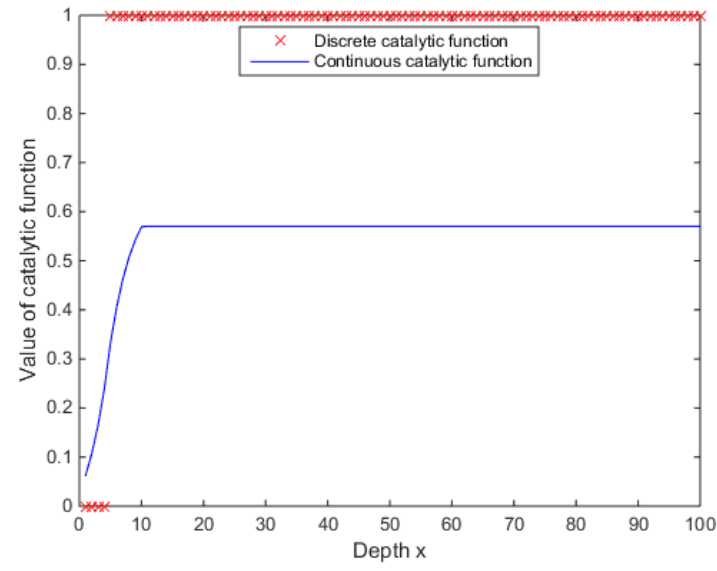

(b) The local optimal catalytic function by Greedy algorithm before pore reaches to $0.86, \mathrm{~J}=100$

Figure 5.14: The transformation from local optimal discrete catalytic functions by Greedy algorithm in Problem $I I_{b}$ before pore reaches to 0.1 and 0.86 to continuous ones, with $\sigma_{\text {trans }}=7 \Delta x$ in (5.49) 


\subsubsection{Comparing the two catalyst positioning models: $\operatorname{cat}(\mathbf{x})$ and $\operatorname{cat}(\mathbf{t}, \mathbf{x})$}

We show the numerical tests on the center-catalyst positioning for the two models: $\operatorname{cat}(x)$ and $\operatorname{cat}(t, x)$. The growth of discharged product $\mathrm{Li}_{2} \mathrm{O}_{2}$ is presented as following As we have seen in the Figure 5.15,
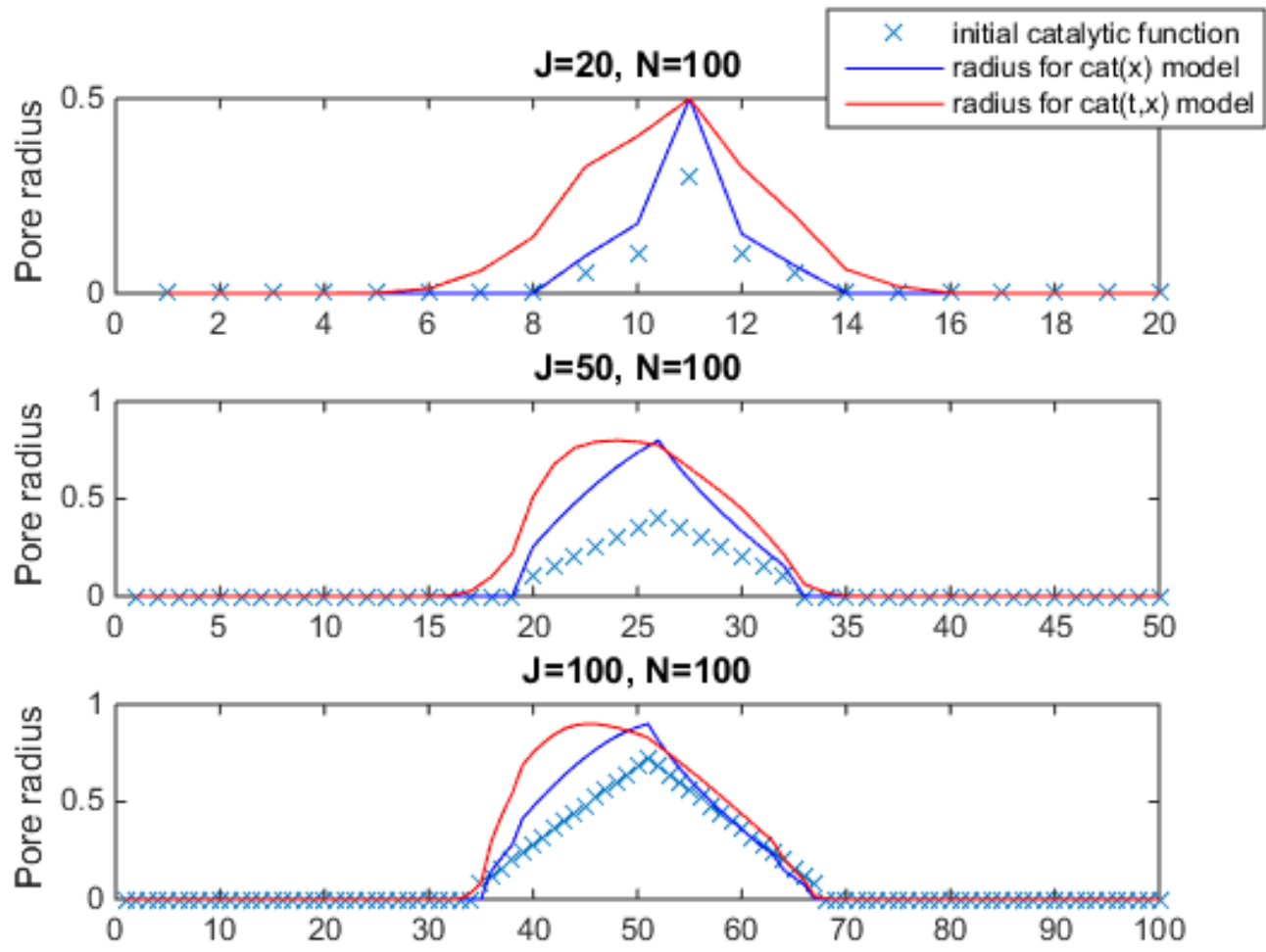

Figure 5.15: Comparing the two catalyst positioning models, experimenting with the center-catalyst positioning. The crosses show the catalytic function at the initial time. Blue lines present the growth profiles of discharged product $\mathrm{Li}_{2} \mathrm{O}_{2}$ deposited along the pore surface after pore clogging in Problem $I_{a}$. The red lines are the growth of $\mathrm{Li}_{2} \mathrm{O}_{2}$ according to Problem $I_{b}$.

in the the $\operatorname{cat}(t, x)$ model, the the growth of $\mathrm{Li}_{2} \mathrm{O}_{2}$ is increasing more to the side where oxygen entering from the left, as the oxygen concentration and deposition rate duo to the electrochemical reaction are higher on the left than on the right sight.

\subsection{The optimization problems}

We will solve numerically the two optimization problems use alternatively. We rely on the line search method, e.g. in [26]. Suppose that $c a t^{k}$ is given, $\mathrm{cat}^{k+1}$ is updated by

$$
c a t^{(k+1)}=c a t^{(k)}-\alpha^{(k+1)} V_{c a t}
$$


for the $\operatorname{cat}(x)$ model. The optimization problems for cat $(\mathrm{x}, \mathrm{t})$ model is solved by the iterative method

$$
c a t^{(k+1)}=c a t^{(k)}-\theta^{(k+1)} V_{c a t_{0}},
$$

where $c a t^{k}$ is given. The iteration stops when the free volume at the certain time step $V\left(c a t^{k+1}\right)$ is greater than the previous one $V\left(c a t^{k}\right)$. Even though the $\operatorname{cat}(x, t)$ model has the advantage in describing the evolution of the Cathode surface with the sparse catalytic distribution, for the sake of simplicity, we present the numerical results here only for cat $(\mathrm{x})$ model. By choosing the smooth and "less" sparse distributed catalytic initial function $\operatorname{cat}(x)$, the difference of the performance between the two models will be accepted.

\subsubsection{The first optimization problem for Problem $I_{a}$}
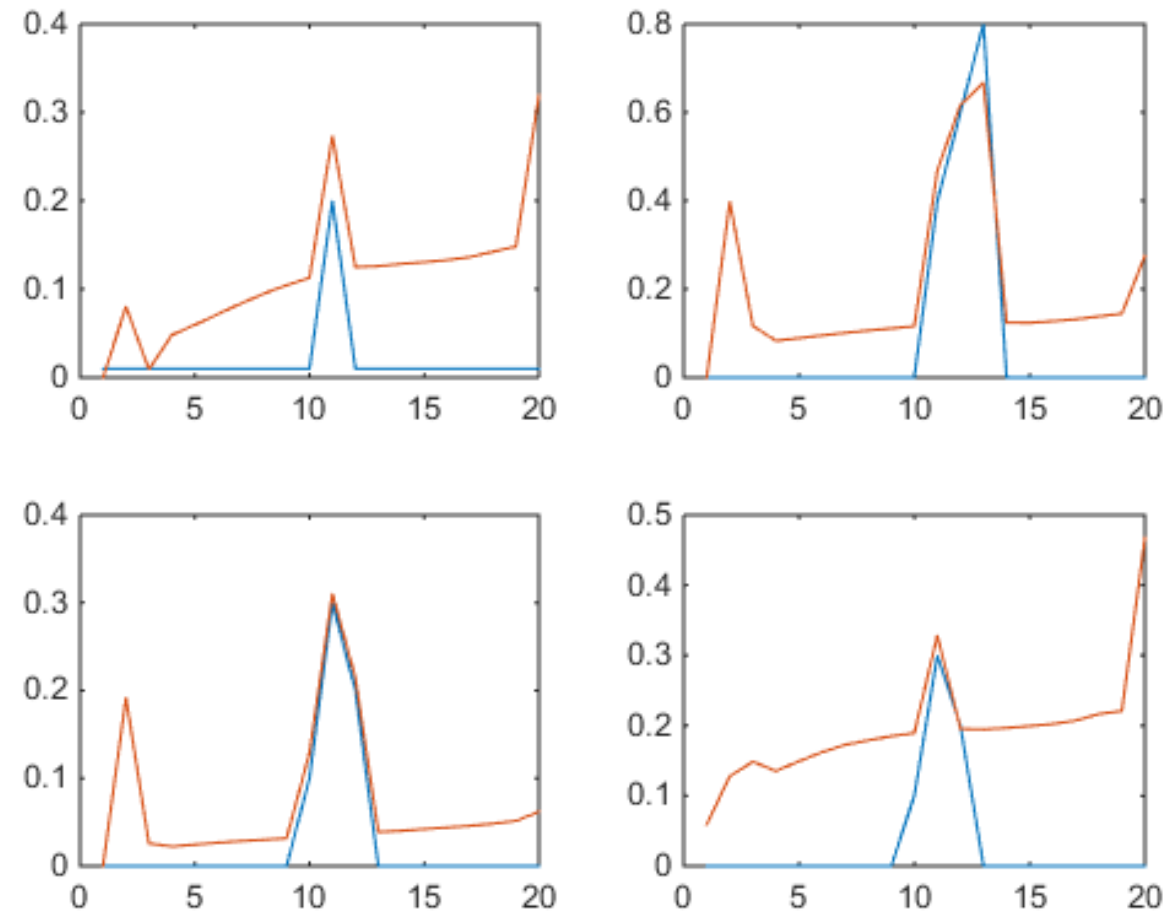

Figure 5.16: Initial $\operatorname{cat}(x)$ and optimal $\operatorname{cat}(x)$ for Problem $I_{a}$ in grid of $J=N=20$

At the first optimization problem, we consider the minimization problem of remaining pore volume with $T, 0<T<\infty$ is the some final time:

$$
\min _{c a t \in \mathbf{A}} V(c a t)=\min _{c a t \in \mathbf{A}} \int_{0}^{1} r^{2}(x, \operatorname{cat}(x), T) d x,
$$

where $A$ is the admission set of catalytic function $\operatorname{cat}(x)$. According to Theorem 4.3

$$
\left(\mathbf{P}_{T} r_{c a t}\right)^{*} \mathbf{P}_{T} r(c a t)
$$


Suppose that $\mathbf{P}_{T}^{(k)} r_{c a t}^{(k)}$ is approximated for $\mathbf{P}_{T} r_{\text {cat }}$ at the iteration $k$ of the optimization algorithm, then $\mathbf{P}_{T}^{(k)} r_{c a t}^{(k)}$ is a $J \times J$ matrix, therefore the adjoint operator is obviously defined by its transpose matrix $\left(\mathbf{P}_{T}^{(k)} r_{c a t}^{(k)}\right)^{*}=\left(\mathbf{P}_{T}^{(k)} r_{c a t}^{(k)}\right)^{T}$. However, this method of defining $V_{c a t}$ is very much time-consuming as we have to calculate $J$ directive derivatives in order to get the Fréchet derivative. This progress is relied on the sensitivity system from Theorem 4.14 . We rewrite the sensitivity system from previous Chapter

$$
\eta_{t}+\frac{D_{2} c a t \varphi}{\int_{0}^{1} r c d x}-\frac{D_{2} c a t c}{\left(\int_{0}^{1} r c d x\right)^{2}} \int_{0}^{1} r \varphi+c \eta d x=\frac{D_{2} \delta c}{\int_{0}^{1} r c d x},
$$

with the initial condition

$$
\begin{gathered}
\eta(0, x)=0, \\
\varphi_{t}-\varphi_{x x}-\frac{2 r_{x} \varphi_{x}}{r}+\left(\frac{2 r_{t}}{r}+\frac{D_{1}}{r \int_{0}^{1} r c d x}\right) \varphi=\frac{2 c_{x} \eta_{x}}{r}-\frac{2 c \eta_{t}}{r} \\
+\left(\frac{2 r_{t} c}{r^{2}}-\frac{2 r_{x} c_{x}}{r^{2}}+\frac{D_{1} c}{r^{2} \int_{0}^{1} r c d x}\right) \eta+\frac{D_{1} c}{r\left(\int_{0}^{1} r H_{\epsilon}(c) d x\right)^{2}} \int_{0}^{1} c \eta+r c \varphi d x,
\end{gathered}
$$

with initial and boundary conditions

$$
\begin{aligned}
& \varphi(0, x)=0, \\
& \varphi(t, 0)=0, \\
& \varphi_{x}(t, 1)=0 .
\end{aligned}
$$

In order to solve the sensitivity system, we apply FEM, which has been presented for forward problems in Section 5.1, taking $\theta=1$. We want to find $\varphi_{h}^{m+1}=\sum_{j=1}^{J-2} v_{j} \Phi_{j}$ at time step $t^{m+1}$.

$$
S v=b^{s}
$$

where $S$ is a $(J-2) \times(J-2)$ matrix

$$
\begin{gathered}
S=\left[\begin{array}{ccccc}
b b_{1}^{s} & c c_{1}^{s} & 0 & \cdots & 0 \\
a a_{1}^{s} & b b_{2}^{s} & c c_{2}^{s} & \cdots & 0 \\
\cdots & \cdots & \cdots & \cdots & \cdots \\
0 & \cdots & \cdots & b b_{J-3}^{s} & c c_{J-3}^{s} \\
0 & \cdots & \cdots & a a_{J-2}^{s} & b b_{J-2}^{s}
\end{array}\right] \\
v=\left(v_{1}, \cdots, v_{J-2}\right), b^{s}=\left(b_{1}^{s}, \cdots, b_{J-2}^{s}\right)^{T} .
\end{gathered}
$$

$v$ and $b^{s}$ are determined according to the backward Euler and FEM method. Using line search optimization method, we get several results described in the figure 5.16. The grid that we work with for this case is very sparse, only $J=N=20$, and we get some overview on the optimal catalytic function. Here, the blue lines are initial catalytic functions, the red one are the local optimal catalytic functions. The discretization we use 20 points for the spacial direction $(\mathrm{J}=20)$ and $\Delta_{t}=\frac{1}{20-1}$. In this Figure, we can see that there are many local minima of the objective functional. 


\subsubsection{The second optimization problem}

For the convenience to apply the adjoint method we also present the second optimization problem that minimizes the mean value of $V_{\text {free }}(r, c a t, t)$ in a small interval of time $\left[T_{1}, T\right]$ :

$$
\min _{\text {cat } \in \mathbf{A}_{\text {cat }}} \mathcal{V}(\text { cat })=\min _{\text {cat } \in \mathbf{A}} \int_{T_{1}}^{T} \int_{0}^{1} r^{2}(x, \operatorname{cat}(x), t) d x d t,
$$

The advantage of sensitivity method is that the executing time takes very long. Therefore we cannot test with many initial catalytic functions, while with this catalyst positioning problem, the objective functions has many local minimizers. Following Chapter 4, under the assumption $\operatorname{cat}_{x}(1)=0$., The derivative of $V(c a t)$ with respect to $c a t$ is

$$
V_{c a t}=-\int_{0}^{T} \frac{D_{2} v c}{\int_{0}^{1} r c d x} d t
$$

Instead of calculating $J$ directional derivatives by solving the sensitivity system, with this second optimization problem, we only solve once the adjoint system from Theorem 4.16. We use FEM to solve this system. The second optimization problem is applied with $T_{1}=T-\Delta t(M-5)$, where $M$ is number of time steps.

Using the line search algorithm from (5.51), we get numerical result of the adjoint method expressed in the Figures 5.17 and 5.17: The blue lines are initial catalytic functions, the red one are the local optimal catalytic functions. The algorithm considering when the pore radius reaches to 0.81 in the Figure 5.17 and to 0.79 in the Figure 5.18. In both cases, the free Volume of the pore via Adjoint method is below than the one via Greedy method around $3-6.5 \%$. 


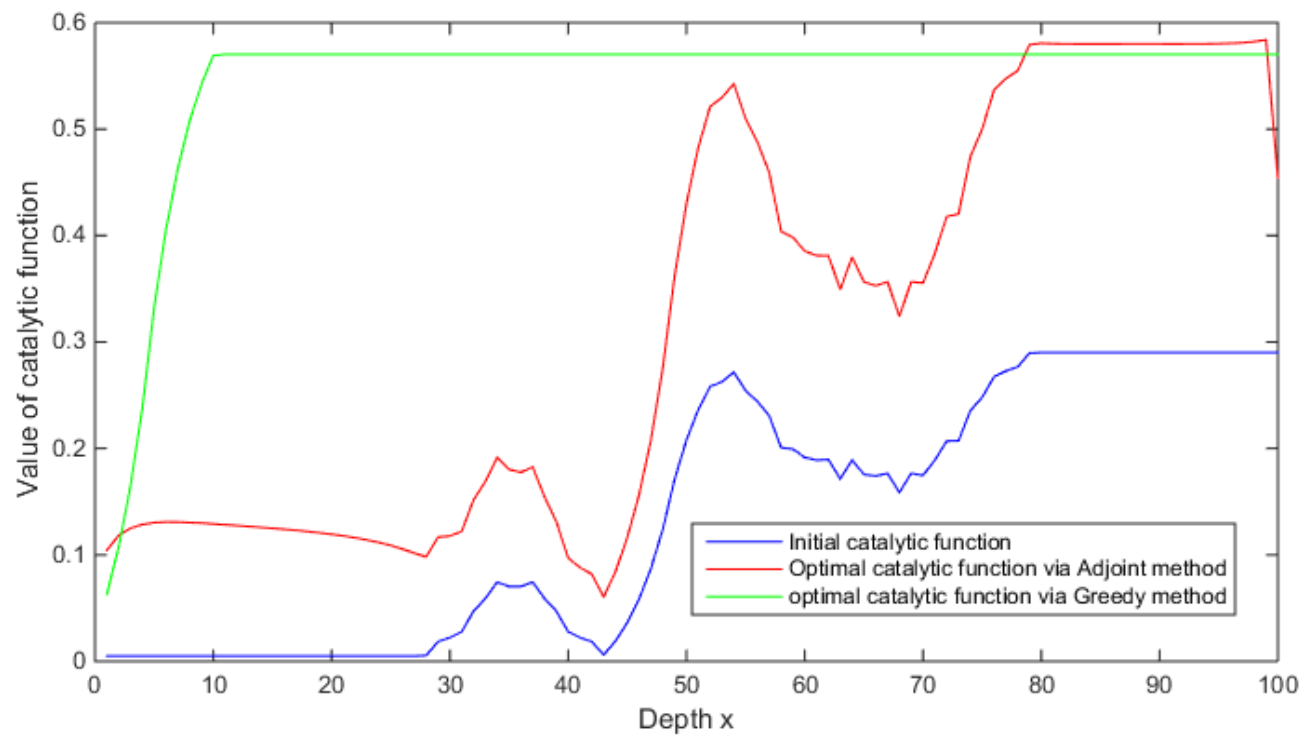

(a) The optimal catalytic function by Adjoint method and by Greedy method

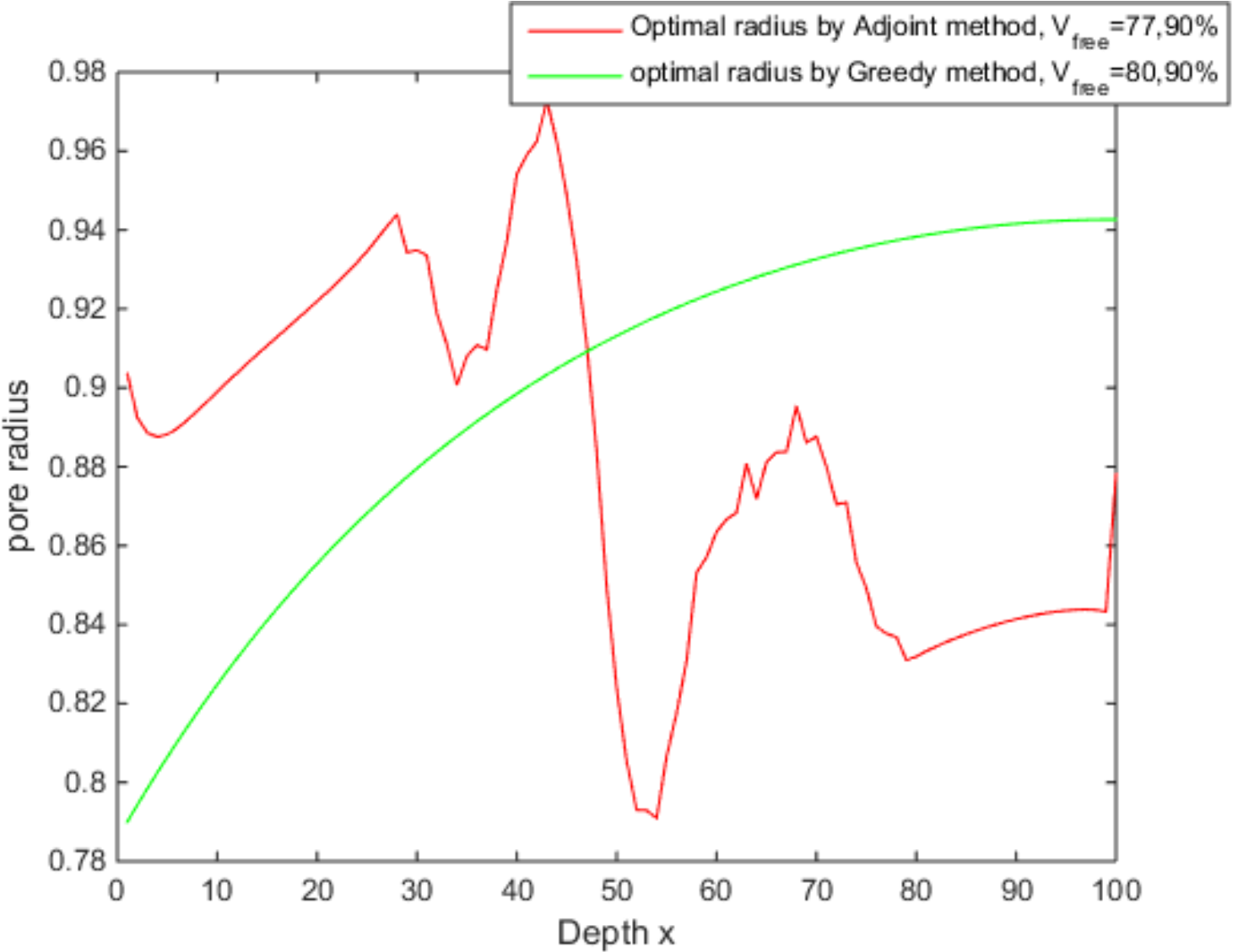

(b) The optimal pore radii via Adjoint method and Greedy method in Problem $I_{a}$. The $\mathrm{Li}_{2} \mathrm{O}_{2}$ is deposited in the upper side in this plot

Figure 5.17: Adjoint method and Greedy method with $\mathrm{J}=100, \mathrm{~N}=80$, number of time steps $\mathrm{M}=1000000$. 


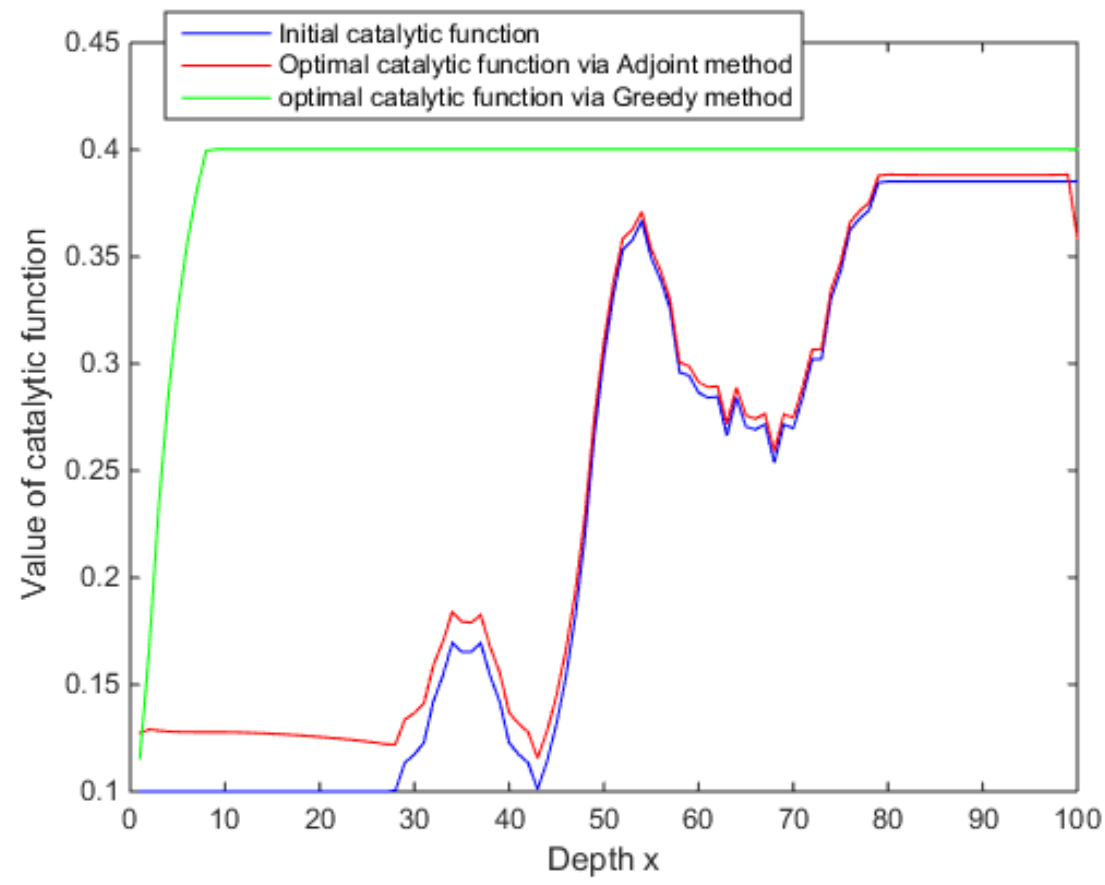

(a) The optimal catalytic function by Adjoint method and by Greedy method

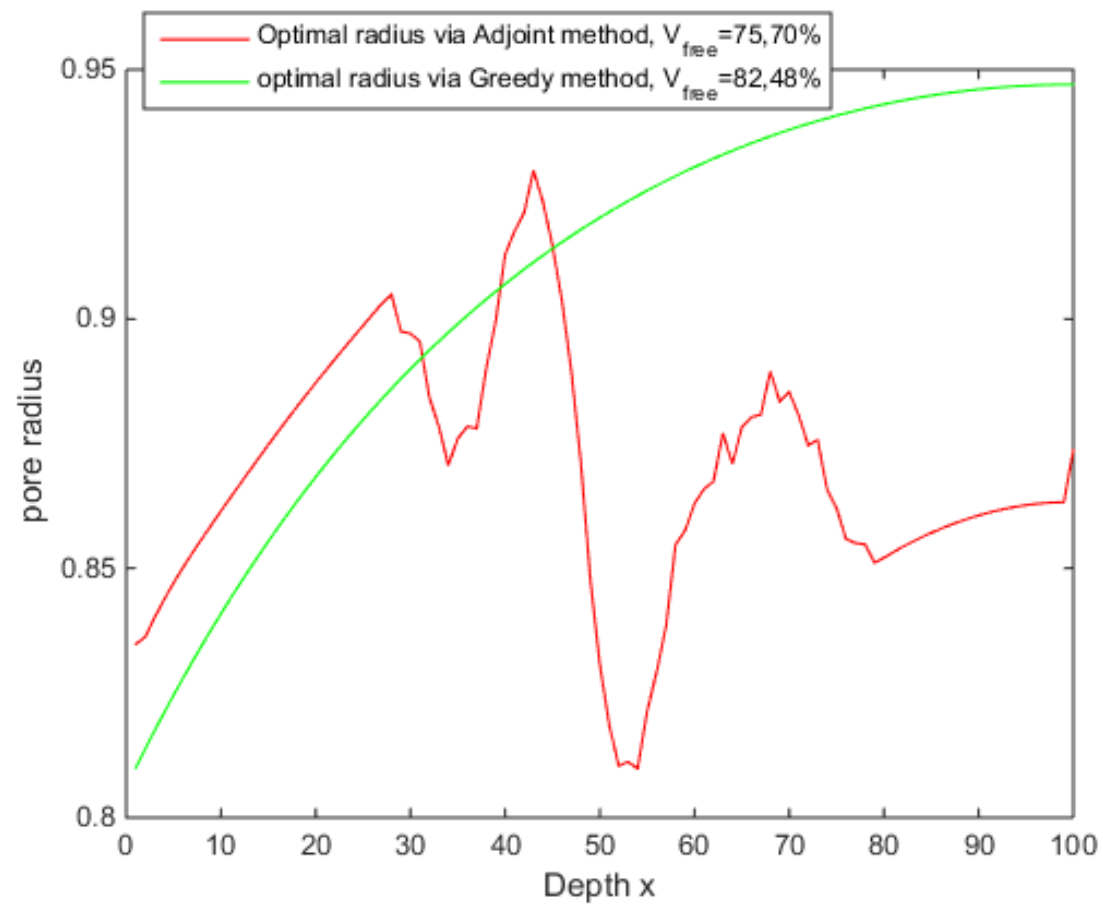

(b) The optimal pore radii via Adjoint method and Greedy method

Figure 5.18: Adjoint method and by Greedy method in Problem $I_{a}$ with $\mathrm{J}=100, \mathrm{~N}=60$, number of time steps $\mathrm{M}=1000000$. The $\mathrm{Li}_{2} \mathrm{O}_{2}$ is deposited in the upper side in this plot 


\section{Conclusions}

In this Thesis, we first have been working with two catalytic positioning model of the reformulated system of the two partial differential equations with initial and mixed boundary conditions. We investigated the well-posedness of the forward problems, the differentiability of the radius and concentration of oxygen inside the pore with respect to catalytic function in $W^{1} \infty(\Omega)$. We also prove the solvability of the two optimization problems. Note that the well-posedness of forward problems is local due to the lack of the comparison principle for the weak solution of parabolic equation including the first derivative of $x$. In the future, we should research the non-local well-posedness of this forward problem. A new approach for the proof of the well-posedness of the original system of the catalytic positioning in $\mathrm{Li}$ /air baterries could be is studying the classical solution, with which comparison principle works. The fixed point argument for classical solution is written in the book of Friedman [14]. In comparison with the greedy algorithm in [8], our catalyst positioning model is more advanced with the "smoother" catalytic functions.

The numerical part illustrates the sensitivity and adjoint methods and shows that these algorithms works. However, there exist some limitations:

1. The sensitivity requires long time to calculate all the directional derivative for every catalytic function.

2. In order to proceed the adjoint method we need to solve the backward problems, which leads us to store all values of pore radius and concentration of oxygen inside the pore at every time step. The number of time steps could be up to hundreds of millions if we want to reach till the pore clogged (the pore radius reaches to 0.1 ).

Therefore, in this Thesis, we deal with the optimization problems only in a small interval of time, when the pore radius reaches to 0.8 .

In further study, catalytic function should also be investigated in the first equation which is based on Fickcian diffusion, as the part $v_{R}\left(C_{O_{2}}, \epsilon, r_{p}\right)$ in (1.4) in the Chapter Introduction represents the rate of $\mathrm{Li}_{2} \mathrm{O}_{2}$ formation per unit of cathode volume. The deposition of $\mathrm{Li}_{2} \mathrm{O}_{2}$ is certainly proceeded strongly where catalyst is positioned.

There is one more possible direction for the future work. It is consideration on the 2 or 3 - dimensional spacial setting for the pore to gain a deeper understanding about the problems of maximizing capacity of the $\mathrm{Li} /$ air batteries during its charging process. 



\section{Appendix A}

\section{Elementary Theorems, Lemmas and other concepts}

\section{A.1 Elementary Lemmas and Theorems}

Theorem A.1 [Banach Fixed-Point Theorem (1922)] Suppose that

(i) we are given an operator $\mathcal{T}: \boldsymbol{M} \subseteq \mathbf{X} \longrightarrow \boldsymbol{M}$, i.e. $\boldsymbol{M}$ is mapped into itself by $\mathcal{T}$

(ii) $\boldsymbol{M}$ is a closed non-empty set in a complete metric space $(\mathbf{X}, d)$;

(iii) $\mathcal{T}$ is a k-contrative, i.e.,

$$
d(\mathcal{T}(x), \mathcal{T}(y)) \leq k d(x, y)
$$

for all $x, y \in M$ and for a fixed $k, 0 \leq k<1$.

Then

1. Equation $\mathcal{T}(x)=x, x \in M$ has exactly one solution.

2. The sequence of successive iterations

$$
x_{n+1}=\mathcal{T} x_{n}, n=0,1,2, \cdots
$$

with arbitrary $x_{0} \in \boldsymbol{M}$ converges to the solution $x$.

Proof. We refer [39] for the proof.

Lemma A.2 (Gronwall's inequality (integral form)). [16] Let $\alpha(t), \beta(t)$ and $u(t)$ be real-valued functions on $[0, T]$. Assume that $\beta$ and $u$ are continuous and that the negative part of $\alpha$ is integrable on 
every closed and bounded subinterval of $[0, T]$. If $\beta$ is non-negative and $u(t)$ satisfies the inequalities

$$
u(t) \leq \alpha(t)+\int_{0}^{t} \beta(s) u(s) d s, t \in[0, T]
$$

then

$$
u(t) \leq \alpha(t)+\int_{0}^{t} \alpha(s) \beta(s) \exp \left(\int_{s}^{t} \beta(r) d r\right) d s, t \in[0, T]
$$

Lemma A.3 (Holder's inequality). [12] Assume $1 \leq p, q \leq \infty, \frac{1}{q}+\frac{1}{p}=1$. Then if $u \in L^{p}(\Omega)$, $v \in L^{q}(\Omega)$, we have

$$
\int_{\Omega}|u v| d x \leq\|u\|_{L^{p}(\Omega)}\|v\|_{L^{q}(\Omega)} .
$$

Lemma A.4 ( $\epsilon$-Cauchy inequality). [12]

$$
a b \leq \epsilon a^{2}+\frac{b^{2}}{\epsilon}(a, b>0, \epsilon>0)
$$

Lemma A.5 (Poincare's inequality for $H_{0}^{1}(\Omega)$ ). There exists a constant $C$ depending on $\Omega$ such that with $u \in H_{0}^{1}(\Omega)$, we have

$$
\|u\|_{L^{2}(\Omega)} \leq C\|\nabla u\|_{L^{2}(\Omega)}
$$

Proof. Using Holder's inequality, we have

$$
u^{2}(x)=\left(\int_{\partial \Omega} u_{x}(x) d x\right)^{2} \leq C(\Omega) \int_{\partial \Omega}\left(u_{x}\right)^{2} d x
$$

Hence we deduce the results.

Remark A.6 (Other necessary inequalities). Suppose $u \in L^{\infty}(Q)$, then the following inequalities hold

$$
\begin{gathered}
\|u\|_{L^{2} Q} \leq \sqrt{T}\|u\|_{L^{2}\left(0, T ; L^{\infty}(\Omega)\right)}, \\
\|u\|_{L^{2}(Q)} \leq \sqrt{T}\|u\|_{L^{\infty}(Q)} .
\end{gathered}
$$

\section{A.2 Huber functions}

\section{A.2.1 Continuously differentiable Huber $C^{1}$ function}

We present Huber functions, that we will need further for building a continuous propaganda model of catalyst problem within the pore, and for reforming problem. According to [10] the continuously 


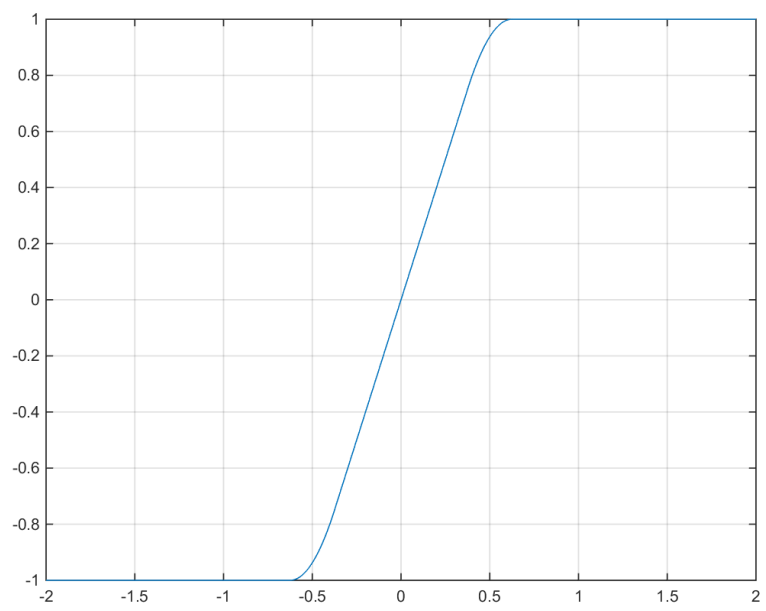

Figure A.1: The graphic of Huber $C^{1}$ function $h_{1, \gamma}(x)$ with $g=1$ and $\gamma=2$

differentiable Huber function is given by

$$
h_{1, \gamma}(x)=\left\{\begin{array}{l}
g, \text { if } x>\frac{g}{2 \gamma}+\frac{1}{2 \gamma^{2}}, \\
g-\frac{\gamma}{2}\left(g-\gamma x+\frac{1}{2 \gamma}\right)^{2}, \text { if } \frac{g}{2 \gamma}-\frac{1}{2 \gamma^{2}}<x \leq \frac{g}{2 \gamma}+\frac{1}{2 \gamma^{2}}, \\
\gamma x, \text { if }-\frac{g}{2 \gamma}+\frac{1}{2 \gamma^{2}}<x \leq \frac{g}{2 \gamma}-\frac{1}{2 \gamma^{2}}, \\
-\left(g-\frac{\gamma}{2}\left(g+\gamma x+\frac{1}{2 \gamma}\right)^{2}\right), \text { if }-\frac{g}{2 \gamma}-\frac{1}{2 \gamma^{2}}<x \leq-\frac{g}{2 \gamma}+\frac{1}{2 \gamma^{2}}, \\
-g, \text { if } x \leq-\frac{g}{2 \gamma}-\frac{1}{2 \gamma^{2}} .
\end{array}\right.
$$

The plot of $h_{1, \gamma}(x)$ when $g=1$ and $\gamma=2$ is shown in the Figure A.1. In particular, the following properties are satisfied:

- $h(1, \gamma): \mathbb{R} \rightarrow \mathbb{R}$ is monotone, Lipschitz continuous and differentiable.

- $\|h(1, \gamma)(x)\| \leq g$, for $\forall \gamma>0$ and $\forall x \in \mathbb{R}$

Using translation along the axis $O x, O y$, we get

$$
h_{1, \gamma}^{*}(x)=\left\{\begin{array}{l}
2 g, \text { if } x>\frac{g}{2 \gamma}+\frac{1}{2 \gamma^{2}}+R_{0}, \\
2 g-\frac{\gamma}{2}\left(g-\gamma x+\gamma R_{0}+\frac{1}{2 \gamma}\right)^{2}, \text { if } \frac{g}{2 \gamma}-\frac{1}{2 \gamma^{2}}+R_{0}<x \leq \frac{g}{2 \gamma}+\frac{1}{2 \gamma^{2}}+R_{0}, \\
g+\gamma x-\gamma R_{0}, \text { if }-\frac{g}{2 \gamma}+\frac{1}{2 \gamma^{2}}+R_{0}<x \leq \frac{g}{2 \gamma}-\frac{1}{2 \gamma^{2}}+R_{0}, \\
\frac{\gamma}{2}\left(g+\gamma x-\gamma R_{0}+\frac{1}{2 \gamma}\right)^{2}, \text { if }-\frac{g}{2 \gamma}-\frac{1}{2 \gamma^{2}}+R_{0}<x \leq-\frac{g}{2 \gamma}+\frac{1}{2 \gamma^{2}}+R_{0}, \\
0, \text { if } x \leq-\frac{g}{2 \gamma}-\frac{1}{2 \gamma^{2}}+R_{0} .
\end{array}\right.
$$


Taking axial symmetry via $x=0.94$, we get

$$
H_{1, \gamma, R_{0}}(x)=\left\{\begin{array}{l}
0, \text { if } x>\frac{g}{2 \gamma}+\frac{1}{2 \gamma^{2}}+R_{0} \\
\frac{\gamma}{2}\left(g-\gamma x+\gamma R_{0}+\frac{1}{2 \gamma}\right)^{2}, \text { if } \frac{g}{2 \gamma}-\frac{1}{2 \gamma^{2}}+R_{0}<x \leq \frac{g}{2 \gamma}+\frac{1}{2 \gamma^{2}}+R_{0} \\
g-\gamma x+\gamma R_{0}, \text { if }-\frac{g}{2 \gamma}+\frac{1}{2 \gamma^{2}}+R_{0}<x \leq \frac{g}{2 \gamma}-\frac{1}{2 \gamma^{2}}+R_{0} \\
2 g-\frac{\gamma}{2}\left(g+\gamma x-\gamma R_{0}+\frac{1}{2 \gamma}\right)^{2}, \text { if }-\frac{g}{2 \gamma}-\frac{1}{2 \gamma^{2}}+R_{0}<x \leq-\frac{g}{2 \gamma}+\frac{1}{2 \gamma^{2}}+R_{0} \\
1, \text { if } x \leq-\frac{g}{2 \gamma}-\frac{1}{2 \gamma^{2}}+R_{0},
\end{array}\right.
$$

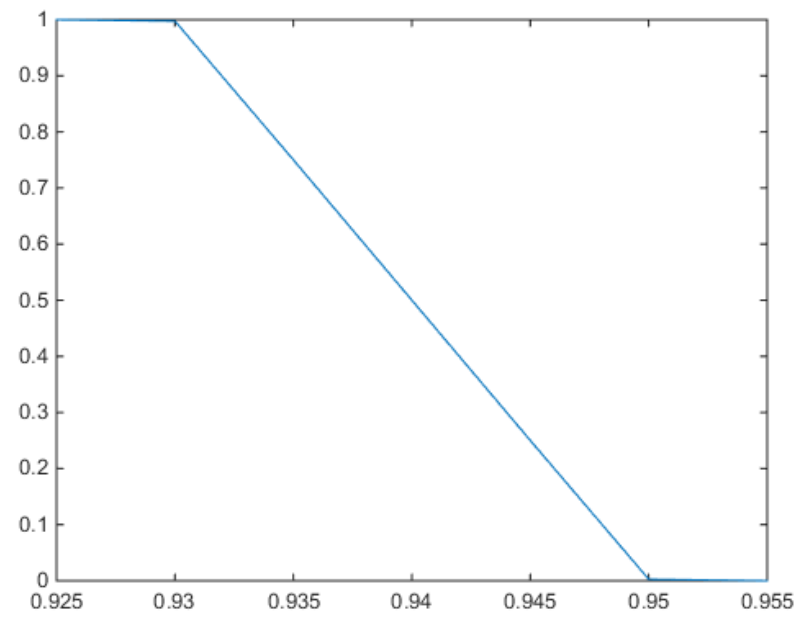

Figure A.2: The graphic of $C^{1}$ Huber function $H_{1, \gamma, R_{0}}(x)$ with $g=1 / 2, \gamma=50$, and $R_{0}=0.94$ Equation (A.9) has plot given in the Figure A.2 with $g=1 / 2, \gamma=50, R_{0}=0.94$

\section{A.2.2 Continuously differentiable $C^{2}$ Huber function}

Referring [6], we present the $C^{2}$ Huber function $h_{2, \gamma}: \mathbb{R} \rightarrow \mathbb{R}$, that is given by:

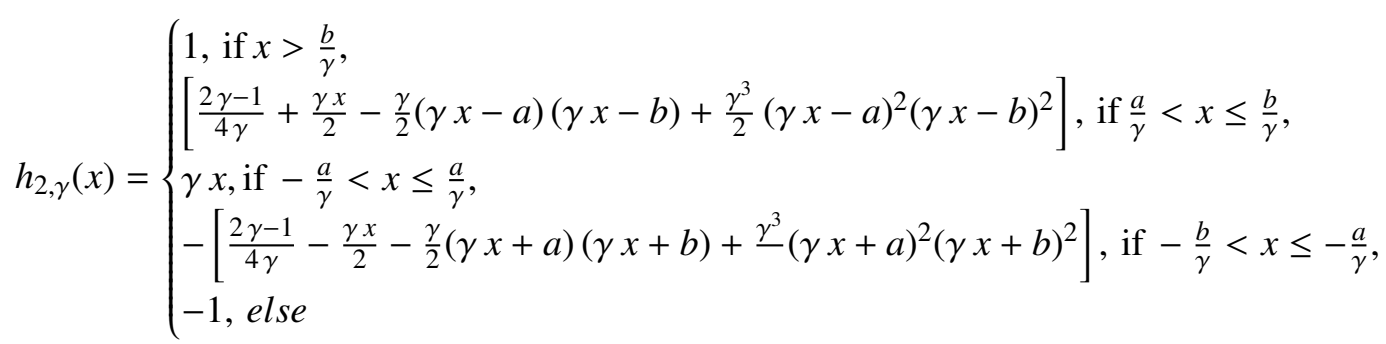

where $a:=1-\frac{1}{2 \gamma}, b:=1+\frac{1}{2 \gamma}$. The graphic of $h_{2, \gamma}(x)$ is given in Figure A.3.

We also have a characteristic of this $C^{2}$ Huber function as following: 


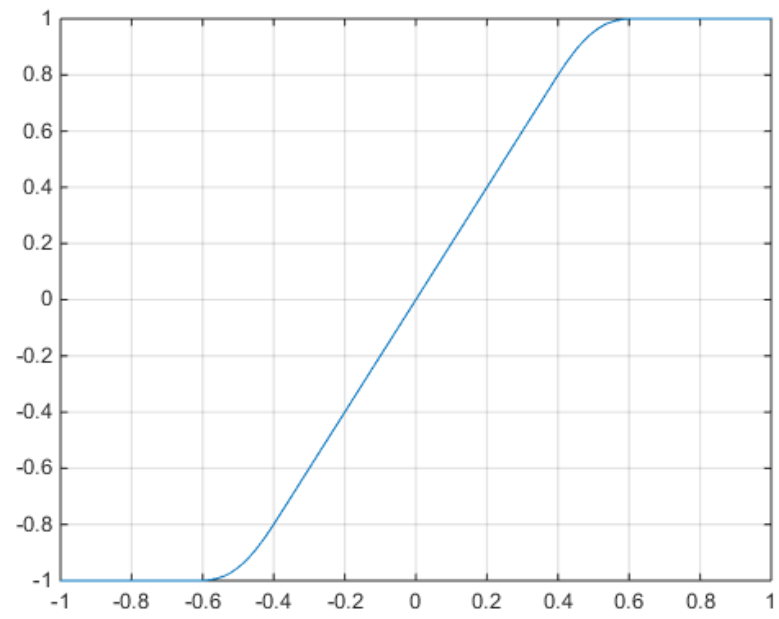

Figure A.3: Graphic of $C^{2}$ Huber function with $\gamma=2$

Lemma A.7 The first and second derivative of the function $h_{2, \gamma}: \mathbb{R} \rightarrow \mathbb{R}$ are Lipschitz continuos functions, with Lipschitz constants depending only on $\gamma$.

Relying on the form of $h_{2, \gamma}(x)$, we would like to deduce a $C^{2}$ function $H(2, \gamma)$ that is increase, always greater that $\epsilon$, taking the value of $x$, where $x$ greater than $c_{0}$. The condition on $c_{0}$ and $\epsilon$ here is:

$$
0<\epsilon<c_{0}<1
$$

$H_{2, \gamma}(x)=\left\{\begin{array}{l}x, \text { if } x \geq-\frac{a}{\gamma}+c_{0} \\ 2 \epsilon-\epsilon\left[\frac{2 \gamma-1}{4 \gamma}-\frac{\gamma x}{2}-\frac{\gamma}{2}\left(\gamma x-\gamma c_{0}+a\right)\left(\gamma x-\gamma c_{0}+b\right)+\frac{\gamma^{3}}{2}\left(\gamma x-\gamma c_{0}+a\right)^{2}\left(\gamma x-\gamma c_{0}+b\right)^{2}\right], \\ \text { if }-\frac{b}{\gamma}+c_{0}<x \leq-\frac{a}{\gamma}+c_{0} \\ \epsilon, \text { else }\end{array}\right.$

where $\epsilon=\frac{1}{\gamma}$ and $c_{0}=\frac{1}{\gamma}$. Graphic of $H_{2, \gamma}(x)$ is expressed in the Figure A.4.

\section{A.3 The Gaussian distribution (Normal distribution)}

In the introduction, we recall the discrete propagation model for the deposited $\mathrm{Li}_{2} \mathrm{O}$ during the pore of the cathode in [8]. In this work we would like to introduce a continuous propagation model based on Gaussian distribution. There are some several reasons why we choose this distribution for describing the propagation among the active surface of the pore: the central natural distribution of data that could express the propagation of the increasing oxygen reaction product among neighborhood and its convenience to use in mathematical calculation. For more understanding on this distribution, we refer 


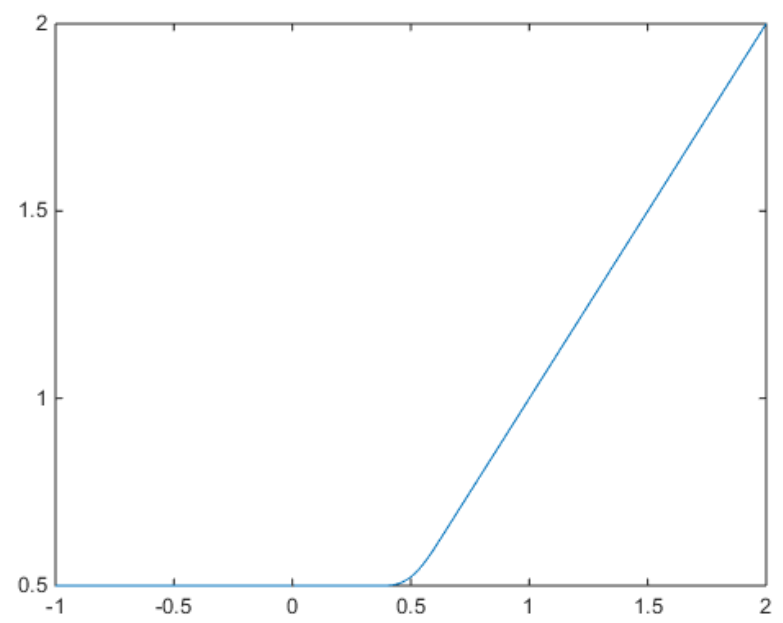

Figure A.4: Graphic of $C^{2}$ function $H_{2, \gamma}(x)$ with $\gamma=2, \epsilon=\frac{1}{\gamma}, c_{0}=\frac{1}{\gamma}$

readers to a text book on statistics like the one from Hogg and his colleagues [18].

Definition A.8 We say a random variable $X$ has a normal distribution if its probability density function (pdf) is

$$
f(x)=\frac{1}{\sqrt{2 \pi} \sigma} \exp \left\{-\frac{1}{2}\left(\frac{x-\mu}{\sigma}\right)\right\},-\infty<x<\infty .
$$

The parameters $\mu$ and $\sigma^{2}$ are the mean and variance of $X$, respectively. We write that $X$ has a $N\left(\mu, \sigma^{2}\right)$ distribution.

It is easy to see that the integral in (A.13) exist and bounded. Moreover the integral is equal to 1.

The graph in Figure A.5 shows that the normal pdf has symmetrical characteristics about a vertical axis through $x=\mu$. Besides, it is easily to see that there is approximately 99.7 per cent within three standard deviations from the mean. That means that the data that has normal pdf is distributed mostly among three-standard-deviation difference.

$$
\int_{\mu-3 \sigma}^{\mu-3 \sigma} \frac{1}{\sqrt{2 \pi} \sigma} \exp \left\{-\frac{1}{2}\left(\frac{x-\mu}{\sigma}\right)\right\} \approx 0.997
$$




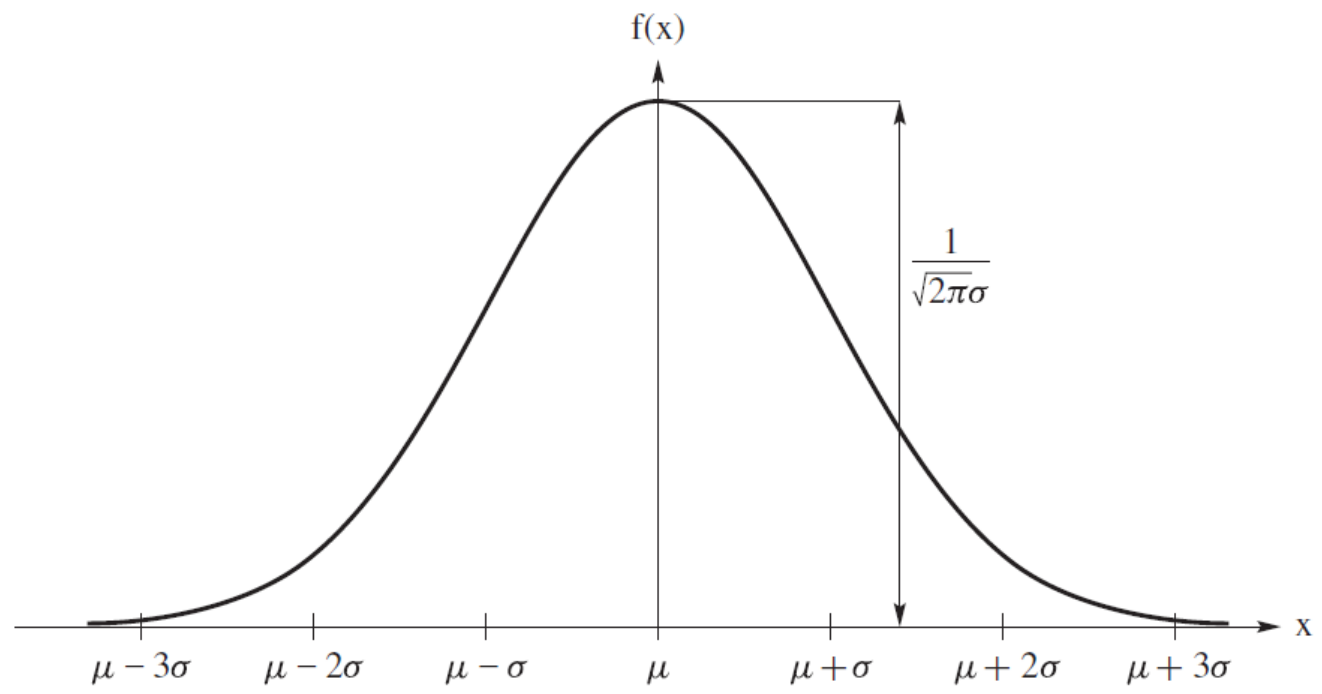

Figure A.5: The normal density $f(x)$

Remark A.9 For the normal distribution that is defined in the definition A.8, we have:

$$
\begin{array}{r}
f(\mu)=\frac{1}{\sqrt{2 \pi} \sigma}, \\
f\left(\mu+\frac{1}{2} \sigma\right)=f\left(\mu-\frac{1}{2} \sigma\right)=\frac{1}{\sqrt{2 \pi} \sigma} \exp \left\{-\frac{1}{4}\right\} \approx 0.7788 \frac{1}{\sqrt{2 \pi} \sigma}, \\
f(\mu+\sigma)=f(\mu-\sigma)=\frac{1}{\sqrt{2 \pi} \sigma} \exp \left\{-\frac{1}{2}\right\} \approx 0.6065 \frac{1}{\sqrt{2 \pi} \sigma}, \\
f\left(\mu+\frac{3}{2} \sigma\right)=f\left(\mu-\frac{3}{2} \sigma\right)=\frac{1}{\sqrt{2 \pi} \sigma} \exp \left\{-\frac{3}{4}\right\} \approx 0.4724 \frac{1}{\sqrt{2 \pi} \sigma}, \\
f(\mu+2 \sigma)=f(\mu-2 \sigma)=\frac{1}{\sqrt{2 \pi} \sigma} \exp \{-1\} \approx 0.3679 \frac{1}{\sqrt{2 \pi} \sigma}, \\
f\left(\mu+\frac{5}{2} \sigma\right)=f\left(\mu-\frac{5}{2} \sigma\right)=\frac{1}{\sqrt{2 \pi} \sigma} \exp \left\{-\frac{5}{4}\right\} \approx 0.2865 \frac{1}{\sqrt{2 \pi} \sigma}, \\
f(\mu+3 \sigma)=f(\mu-3 \sigma)=\frac{1}{\sqrt{2 \pi} \sigma} \exp \left\{-\frac{9}{2}\right\} \approx 0.0111 \frac{1}{\sqrt{2 \pi} \sigma} .
\end{array}
$$





\section{Bibliography}

[1] K.M. Abrham and Z. Jiang. A Polimer electrolyte-based rechargeable Lithium/Oxygen battery. J. Electrochem Soc., 143:1-5, 1996.

[2] M. Aigner. Series in Discrete Mathematics and Theoretical Computer science. american Mathematical Society, 2007.

[3] P. Andrei, P. Zheng, M. Hendrickson, and E. J. Plichta. Some possinle approaches for improving the energy density of Li-Air batteries. Journal of the Electrochemical Society, 157:A1287A1295, 2010.

[4] D. A. G. Bruggeman. Berechnung Verschiedener Physikalisher Konstanten von Heterogenen Substanzen. I. Doeöectrizitätskonstanten ung Leitfähigkeiten der Mischköper aus Isotropen Substanzen. Annalen der Physik, 34:636-664, 1998.

[5] J. Christensen, P. Albertus, R. S. Sanchez-Carrera, T. Lohmann, B. Kozinsky, R. Liedtke, J. Ahmed, and A. Kojic. A Critical Review of Li/Air Batteries. The Journal of Electrochemical society, 159:R1-R30, 2012.

[6] C. Chung, J. C. De Los Reyes, and C. B. Schönlieb. Learning optimal spatially-dependent regularization parameters in total variation image denoising. Inverse Problems, no. 7, 2017.

[7] I. Cioranescu. The Boundary Value Problems of Mathematical Physics. Mathematics and its applications, 1990.

[8] Tatjana Dabrowski, Daniela Fenske, Peter Maass, Alexander Struck, and Lucio Colombi Ciacchi. Optimization of catalyst positioning in porous cathodes of lithium/air batteries filled with different electrolytes. Journal of Power Sources, 2015.

[9] Tatjiana Dabrowski. Atomistic Modeling of the charge process and optimization of catalysts positioning in pourous cathodes of Lithium/air batteries. Doctoral Disertation, University of Bremen, April, 2016.

[10] J. C. De Los Reyes. Optimization of mixed variational inequalities arising in flow of viscoplastic materials. Comput Optim Appl, 2011.

[11] Hao Dinh-Nho, Viet Huong Bui, Xuan Thanh Phan, and D. Lesnic. Identification of nonlinear heat transfer laws from boundary observations. Applicable Analysis, 94:9:1784-1799, 2015. 
[12] Lawrence C. Evans. Partial Differential Equations. Graduate Studies in Mathematics. American Mathematical Society, 1991.

[13] J. Feinauer, S. Hein, S. Rave, S. Schmidt, D. Westhoff, J. Zausch, O. Iliev, A. Latz, M. Ohlberger, and V. Schmidt. MULTIBAT: Unified workflow for fast electrochemical 3D simulations of lithium-ion cells combining virtual stochastic microstructures, electrochemical degradation models and model order reduction. Journal of Computational Science, 2018.

[14] Avner Friedman. Partial differential equations of parabolic type. Robert E. Krieger Publishing company Malabar, Florida, 1983.

[15] Thies Gerken and Armin Lechleiter. Reconstruction of a time-dependent potential from wave measurements. Inverse Problems, 33, 2017.

[16] Thomas H. Gronwall. Note on the derivatives with respect to a parameter of the solutions of a system of differential equations. Ann. of Math, 20:292-296, 1919.

[17] Simon Grutzner. An approach to parameter identification in continuum damage mechanics. Master Thesis, University of Bremen, Germany, 2015.

[18] Robert V. Hogg, Joseph W. McKean, and Allen T. Craig. Introduction to mathematical statistics. 1995.

[19] E. M. LaBolle, J. Quastel, and G. E. Fogg. Transition-Probability Densities of Diffusion Process corresponding to Advection Dispersition Equations. Water Resources Research, 34:1685-1693, 1998.

[20] O. A. Ladyzhenskaya. The Boundary Value Problems of Mathematical Physics. Springer-Verlag, New York, 1985.

[21] O. A. Ladyzhenskaya, V. A. Solonnilov, and N. N. Uralceva. Linear and Quasilinear Equations of Parabolic Types. American Mathematical Society, 1968.

[22] O. A. Ladyzhenskaya, V. A. Solonnilov, and N. N. Uralceva. Linear and Quasilinear Equations of Parabolic Types. American Mathematical Society, 1968.

[23] C.O. Laoire, S. Mukerjee, K.M. Abraham, E. J. Plichta, and M. A. Hendrickson. Eluciating the mechanism of oxygen reduction for lithium-air battery applications. The Journal of Physical Chemistry, 46:20127-20134, 2009.

[24] C.O. Laoire, S. Mukerjee, K.M. Abraham, E. J. Plichta, and M. A. Hendrickson. Influence of nonaqueous solvents on the electrochemistry of oxygen in the rechargeable lithium-air battery. The Journal of Physical Chemistry, 19:9178-9186, 2010.

[25] X. Li and A. Faghri. Optimization of the Cathode structure of Lithium-air Batteries based on a two-Dimensional, Transient, Non-Isothermal Model. Journal of the Electrochemical Society, 159:A1747-A1754, 2012.

[26] Jorge Nocedal and Stephen J. Wright. Numerical Optimization. Springer, 1999. 
[27] M. Ohlberger, S. Rave, and F. Schindler. Model Reduction for Multiscale Lithium-Ion Battery Simulation. Conference Numerical Mathematics and Advanced Applications ENUMATH 2015, Ankara, Turkey, pages 317-331, 2016.

[28] M. Ohlberger, S. Rave, S. Schmidt, and S. Zhang. A Model Redution Framewoek for Efficient Simulation of Li-ion Batteries. Conference: Finite Volumes for Complex Application VII, Berlin, pages 695-702, 2014.

[29] J. Read. Characterization of the lithium/oxygen organic electrolyte battery. J. Electrochem Soc., 149:A1190-A1195, 2002.

[30] J. Read, K. Mutolo, M. rvin, W. Behl, J. Wolfenstine, . A. Driedger, and D. Forster. Characterization of the lithium/oxygen organic electrolyte battery. J. Electrochem Soc., 150, 2003.

[31] A Rosch and F. Troltzsch. An optimal control problem arising from the identification of nonlinear heat transfer laws. Arch. Control Sci, 1:183-195, 1992.

[32] S. S. Sandhu, J.P. Fellner, and G. W. Brutchen. Diffusion-limited model for a lithiunm/air battery with an organic electrolyte. Journal of Power Sourcees, 164:365-371, 2007.

[33] Endre Süli. Lecture Notes on Finite Element Method for Partial Differential Equations. Mathematical Institute, University of Oxford, 2012.

[34] Dong Sun, V.S. Manoranjan, and Hong-Ming Yin. Numerical solution for a coupled parabolic equations arising induction heating processes. Discrete and Continuous Dynamical Systems Supplement, pages 956-964, 2007.

[35] Fredi Troltzsch. Optimal Control of Partial Differential Equations. Theory, Methods and Applications. Graduate Studies in Mathematics. American Mathematical Society, 2015.

[36] S. C. Cho Wang. Analysis of air Cathode Performance for Lithium-Air Batteries. Journal of the Electrochemical Society, 160:A1847-A1855, 2013.

[37] J. Wloka. Partial differential equation. Cambridge University Press 1987, 1987.

[38] Eberhard Zeidler. II/A: Linear Monotone Operators. Nonlinear Functional Analysis and Its Applications. Springer, 1989.

[39] Eberhard Zeidler. I: Fixed point Theorems. Nonlinear Functional Analysis and its Applications. Springer, 1992.

[40] C. Q. Zhang, J. P. Zheng, R. Liang, C. Zhang, B. Wang, M. Au, and M. Hendrickson. $\mathrm{MnO} /$ /Carbon Nanotube/Carbon Nanofiber Composite Catalytic Air Electroders for Rechargeable Lithium-air Batteries. Journal of the Electrochemical Society, 158:A822-A827, 2011.

[41] D. Zhang, Z. Fu, Z. Wei, and A. Yu. Polarization of Oxygen Electrode in Rechargeable Lithium Oxygen Batteries. Journal of the Electrochemical Society, 157:A362-A365, 2010. 